\title{
Kindertivity: Usability and Communicability Strategies for Interactive Surfaces and Pre- Kindergarten Children
}

\author{
by \\ Vicente Ernesto Nácher Soler \\ Supervised by \\ Dr. Francisco Javier Jaén Martínez
}

\begin{abstract}
A thesis submitted for the degree of
Doctor of Philosophy in Computer Science
\end{abstract}

Departamento de Sistemas Informáticos y Computación

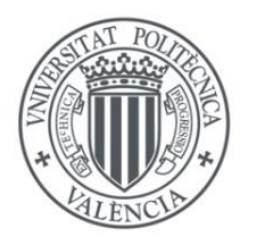

UNIVERSITAT

POLITĖCNICA

DE VALÈNCIA

December, 2018 



\begin{abstract}
Multi-touch technology has become one of the most emergent technologies and has had an enormous growth since its initial steps in the eighties to be widespread accepted and used in the present. On the one hand, multi-touch technology relies on the direct manipulation interaction style which gives users the advantage to view the objects and actions of interest, replace typed commands by pointing actions and to perform rapid, reversible and incremental actions avoiding using complex instructions. On the other hand, several works have evaluated the virtues when joining direct manipulation with direct-touching showing that it solves the problems inherent in other interaction devices, such as those involving mouse or keyboard. Hence, taking advantage of the intuitive and natural interaction provided by multi-touch technology it seems an ideal way to support educational scenarios targeted to kindergarten children. Although several works have assessed the suitability of using the direct manipulation style with children, there is a lack of works addressing the use of touchscreen devices by this specific type of users.
\end{abstract}

Moreover, there is a growing trend of designing educational and playful applications targeted to kindergarten children based on touchscreen devices such as smartphones and tablets. In addition, several reports point out that children use touchscreen devices even before they are able to speak and they are frequent users of devices such as smartphones and tablets.

However, despite this growth in the use of multi-touch technology by children and its apparent suitability to be used to develop applications targeted to young children, there is a lack of standardized and universally accepted interactions for young children when using touchscreen devices since only two basic gestures are commonly used (basically, consisting of only one-finger touch for selection and one-finger drag for movement). Hence, there is a need of carrying out empirical studies to help and advance in the design of applications that adequately support and fit with children's development and skills.

Therefore, this thesis proposes, designs and evaluates several usability and communicability strategies tailored to children in their early development stage to establish the design and development of future applications targeted to kindergarten children. These strategies will lead to define appropriate design strategies that enable infants to take full advantage of multi-touch technology, would make it possible to develop attractive new applications and, eventually, could also aid children's cognitive and motor development. 



\section{Resumen}

La tecnología multi-táctil se ha convertido en una de las más emergentes tras experimentar un enorme crecimiento desde sus pasos iniciales en los años ochenta hasta su amplia aceptación y uso en la actualidad. Por una parte, la tecnología multitáctil se basa en el estilo de interacción de manipulación directa el cual proporciona a los usuarios la ventaja de ver los objetos y las acciones de interés, sustituir comandos escritos por acciones de señalado y, además, permite la realización de acciones rápidas, reversibles e incrementales evitando el uso de instrucciones complejas. Por otra parte, diversos trabajos han evaluado las virtudes derivadas de utilizar conjuntamente la manipulación directa con el toque directo mostrando que es posible evitar los problemas inherentes a otras técnicas de interacción como el ratón y el teclado. Por lo tanto, aprovechando la interacción natural e intuitiva proporcionada por la tecnología multi-táctil, ésta parece una forma ideal para dar soporte a la creación de escenarios educativos dirigidos a niños en edad preescolar. Sin embargo, a pesar de la existencia de diversos estudios que evalúan la idoneidad de utilizar el estilo de interacción de manipulación directa, existe una falta de trabajos abordando el uso dispositivos basados en superficies táctiles con niños de una temprana edad.

Asimismo, en la actualidad existe una creciente tendencia a diseñar aplicaciones educativas y lúdicas dirigidas a niños en edad preescolar utilizando dispositivos multitáctiles como los teléfonos inteligentes o las tabletas. Además, diversos informes señalan que los niños son usuarios frecuentes de este tipo de dispositivos y los utilizan incluso antes de ser capaces de hablar.

Sin embargo, a pesar de este crecimiento en el uso de la tecnología multi-táctil y su aparente idoneidad para ser utilizado en el desarrollo de aplicaciones educativas para niños en edad preescolar, no existen unas interacciones universales y estandarizadas para preescolares a la hora de utilizar dispositivos táctiles ya que habitualmente sólo se utilizan dos gestos básicos (básicamente, el toque con un dedo para seleccionar y el arrastre con un dedo para el movimiento). Por lo tanto, existe una clara necesidad de llevar a cabo estudios empíricos para contribuir y avanzar en el diseño de aplicaciones que den un soporte adecuado y encaje con las habilidades de los niños en su temprano desarrollo.

Por tanto, esta tesis propone, diseña y evalúa diversas estrategias de usabilidad y comunicabilidad adaptadas a los niños en edad preescolar para establecer la base para el diseño y desarrollo de futuras aplicaciones basadas en dispositivos táctiles dirigidas a preescolares. Estas estrategias llevarán a la adecuada definición de guías de diseño que permitirán a los niños aprovechar al máximo la tecnología multi-táctil, harán posible el desarrollo de nuevas y atractivas aplicaciones y, eventualmente, también podrán ayudar al desarrollo cognitivo y motor de los niños. 



\section{Resum}

La tecnologia multi-tàctil s'ha convertit en una de les més emergents després d'experimentar un enorme creixement des dels seus passos inicials als anys vuitanta fins l'actualitat on es àmpliament acceptada i utilitzada. D'una banda, la tecnologia multi-tàctil es basa en l'estil d'interacció de manipulació directa, el qual proporciona als usuaris l'avantatge de veure els objectes i les accions d'interès, substituir comandos escrits per accions d'assenyalament $i$, a més, permet la realització d'accions, ràpides, reversibles i incrementals evitant l'ús d'instruccions complexes. D'altra banda, diversos treballs han avaluat les virtuts derivades d'utilitzar conjuntament la manipulació directa amb el toc directe mostrant que és possible evitar els problemes inherents a altres tècniques d'interacció com el ratolí i el teclat. Per tant, aprofitant la interacció natural i intuïtiva proporcionada per la tecnologia multi-tàctil, aquesta sembla una forma ideal per donar suport a la creació d'escenaris educatius per a xiquets en edat preescolar. No obstant això, malgrat l'existència de diversos estudis que avaluen la idoneïtat d'utilitzar l'estil d'interacció de manipulació directa, existeix una manca de treballs abordant l'ús de dispositius basats en superfícies tàctils amb xiquets d'edat primerenca.

Així mateix, en l'actualitat existeix una creixent tendència a dissenyar aplicacions educatives i lúdiques dirigides a xiquets en edat preescolar utilitzant dispositius tàctils com els telèfons intel-ligents o les tauletes. A més, diversos informes assenyalen que els xiquets són usuaris freqüents d'aquests tipus de dispositius i els utilitzen fins i tot abans de ser capaços de parlar.

Malgrat aquest creixement en l'ús de la tecnologia multi-tàctil i la seua aparent idoneïtat per a ser utilitzada en el desenvolupament d'aplicacions educatives per a xiquets en edat preescolar, no existeixen unes interaccions universals i estandarditzades per a preescolars a l'hora d'utilitzar dispositius tàctils ja que habitualment només s'utilitzen dos gestos bàsics (bàsicament, el toc amb un dit per a seleccionar $\mathrm{i}$ l'arrossegament amb un dit per al moviment). Per tant, hi ha una clara necessitat de dur a terme estudis empírics per a contribuir i avançar en el disseny d'aplicacions que donen un suport adequat i s'ajusten amb les habilitats dels xiquets en el seu primerenc desenvolupament.

Per tant, la tesi proposa, dissenya $\mathrm{i}$ avalua diverses estratègies de usabilitat $\mathrm{i}$ comunicabilitat adaptades als xiquets en edat preescolar per tal d'establir la base per al disseny i desenvolupament de futures aplicacions basades en dispositius tàctils dirigides a preescolars. Aquestes estratègies portaran a l'adequada definició de guies de disseny que permetran als xiquets aprofitar al màxim la tecnologia multi-tàctil, faran possible el desenvolupament de noves i atractives aplicacions i, eventualment, podran també ajudar al desenvolupament cognitiu i motor dels xiquets. 



\section{Keywords}

Children-Computer Interaction, Pre-kindergarten, Touch Interaction, Tablets, Education, Multi-touch Interaction, Gestures, Usability Evaluation, Communicability, Visual Languages, Directional Awareness 



\section{Agradecimientos}

Me gustaría mostrar mi gratitud con todas aquellas personas que me han acompañado durante estos años haciendo esto posible.

A mi familia, por su apoyo y comprensión, y por animarme a llevar a cabo todos mis proyectos.

A todos los compañeros con los que he coincidido en el laboratorio del grupo ISSI, por su amabilidad y por prestarme su ayuda siempre que la he necesitado: Abel, Alejandro, José Antonio, Ana, David, Javi, Miguel Ángel, Priscila y Sandra. En especial, a Fernando, Patricia, Kamil y Alicia que han compartido la mayor parte del camino conmigo siempre creando y participando en interesantes debates en el laboratorio que han ayudado a la realización de esta tesis.

Y por último, dar las gracias a mi director de tesis, Javi, por darme la oportunidad de llevar a cabo este proyecto, por su paciencia, dedicación y esfuerzo durante la tesis siendo el mejor patrón posible para que todo siguiera su rumbo.

Muchas gracias a todos. 



\section{Contents}

Contents.................................................................................................

I Preliminaries 1

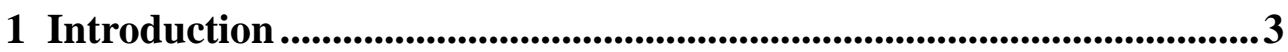

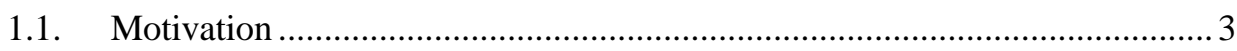

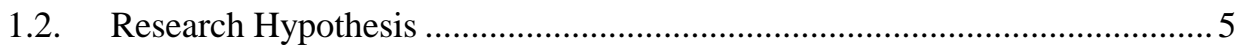

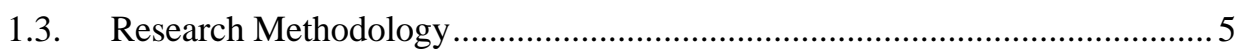

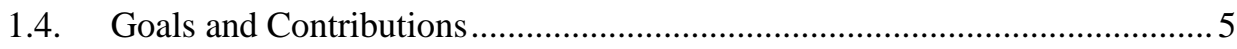

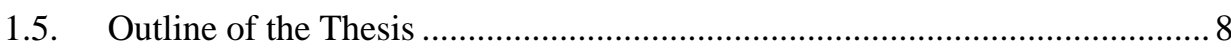

2 Interactive Technologies for Preschool Game-Based Instruction: Experiences and Future Challenges ....................................................................13

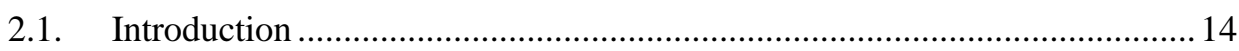

2.2. Technology-Supported Games for Preschool Children.................................. 15

2.2.1. Traditional Computers ..................................................................... 15

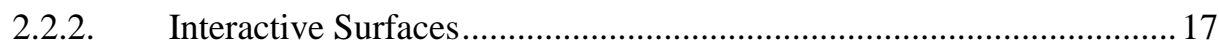

2.2.3. Robots and Technologically-Enhanced Toys.......................................22

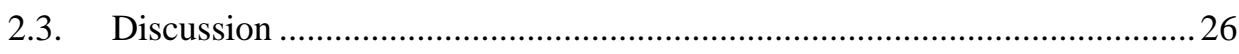

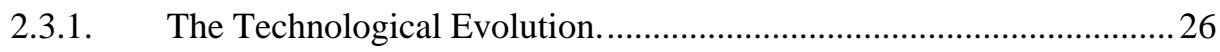

2.3.2. A Future Challenge: Ubiquitous games.............................................2 
Kindertivity: Usability and Communicability Strategies for Interactive Surfaces and PreKindergarten Children

2.3.3. The Interplay between Age, Cognitive Skills and Interaction

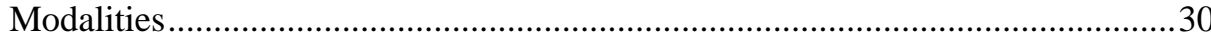

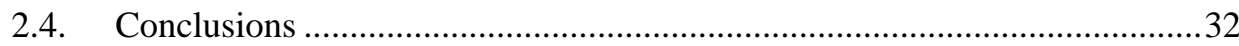

3 Multi-touch Technology in Early Childhood: Current Trends and Future Challenges .....................................................................................33

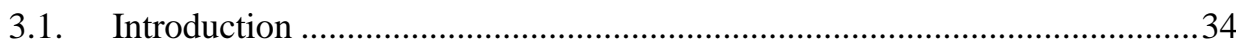

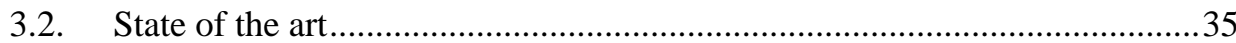

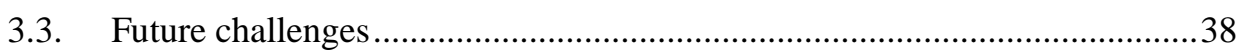

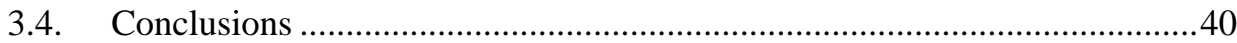

4 KINDERTIVITY: Using Interactive Surfaces to Foster Creativity in Pre-kindergarten Children.......................................................................... 43

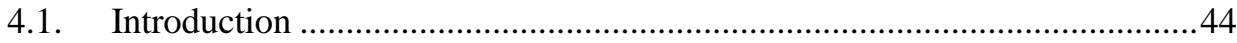

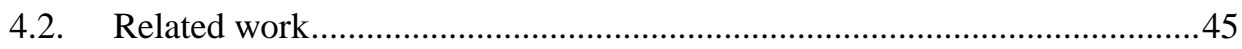

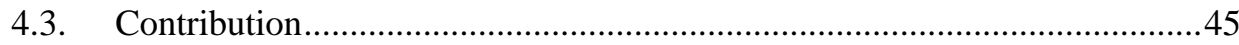

II Usability $\quad 47$

5 Multi-touch Gestures for Pre-kindergarten Children .......................... 49

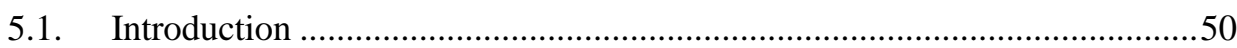

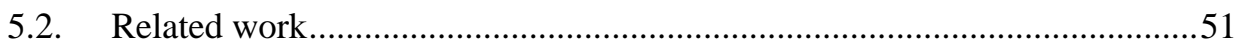

5.2.1. Commercial perspective on multi-touch technology …….....................53

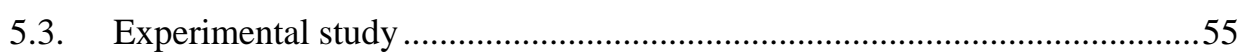

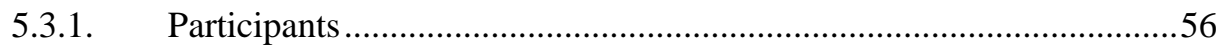

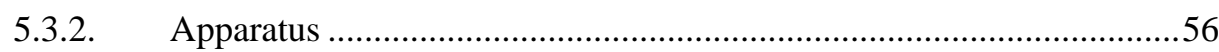

5.3.3. Procedure

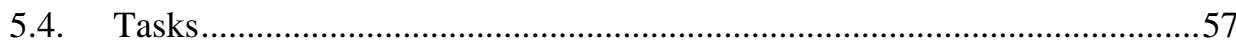

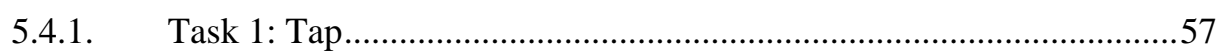

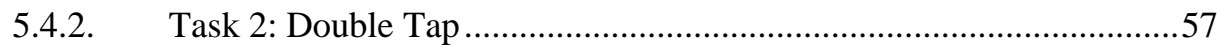

xiv 


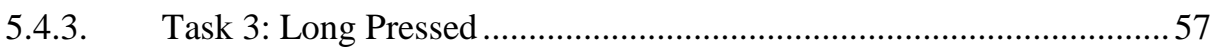

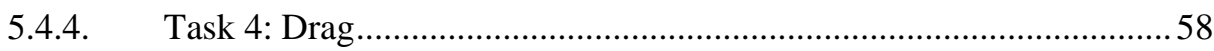

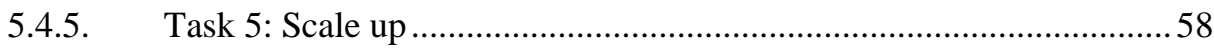

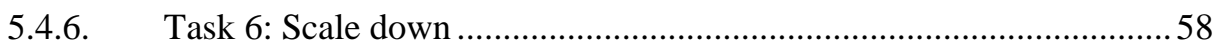

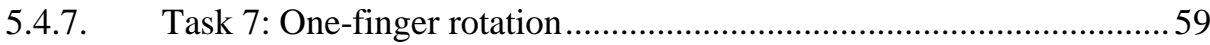

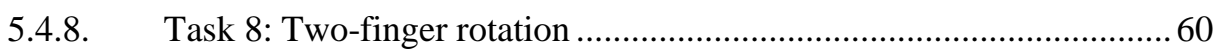

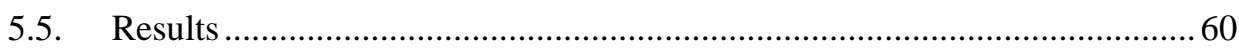

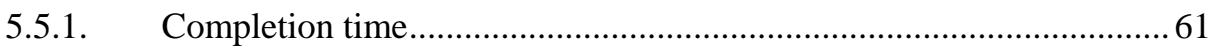

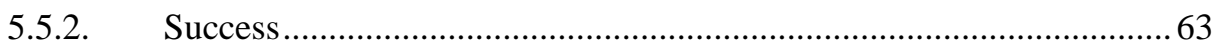

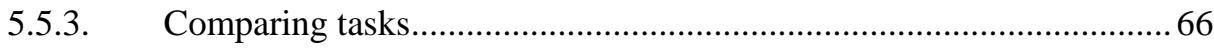

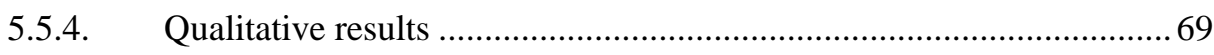

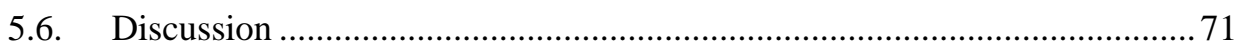

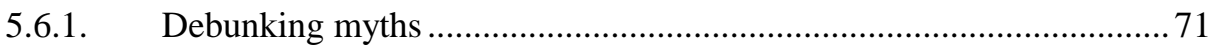

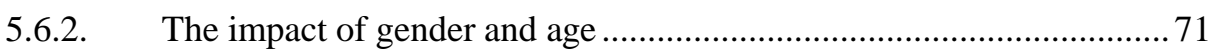

5.6.3. Designing multi-touch applications for pre-kindergarteners................. 72

5.6.4. Revisiting multi-touch interactions for adults ....................................... 73

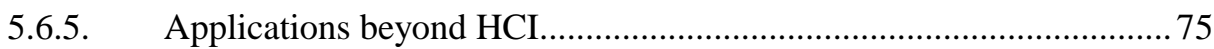

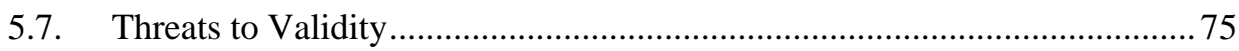

5.8. Conclusions and future work .........................................................................

6 Improving Pre-Kindergarten Touch Performance ............................83

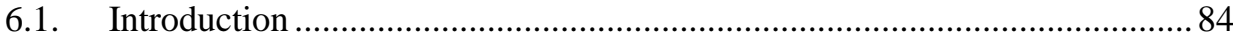

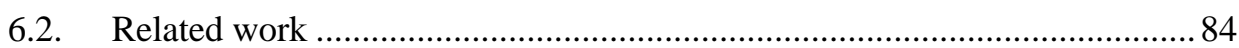

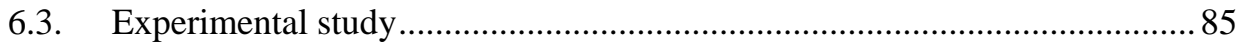

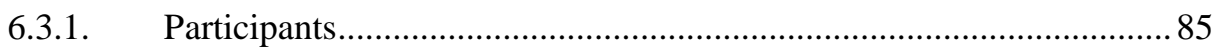

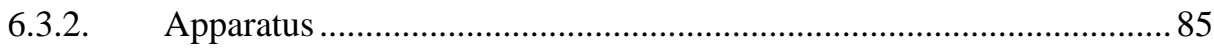

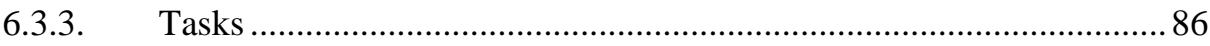

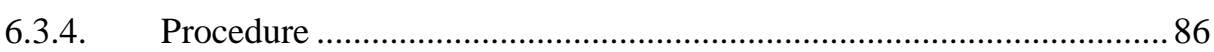

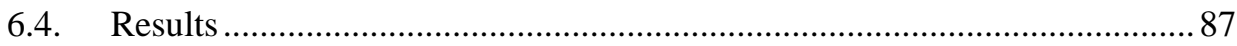

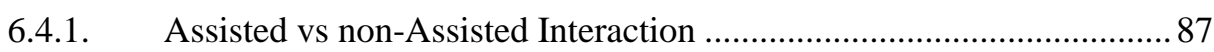


Kindertivity: Usability and Communicability Strategies for Interactive Surfaces and PreKindergarten Children

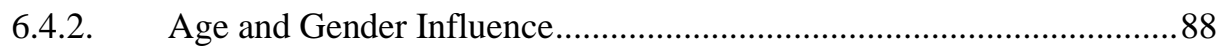

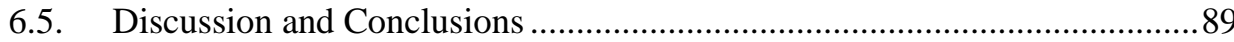

7 Evaluating the Accuracy of Pre-Kindergarten Children Multi-touch Interaction .......................................................................................91

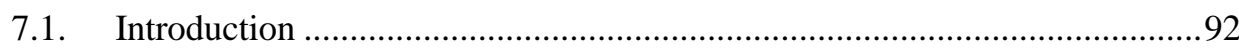

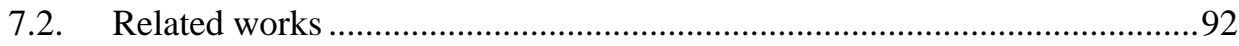

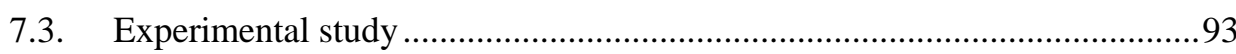

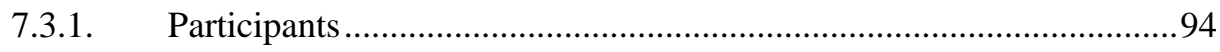

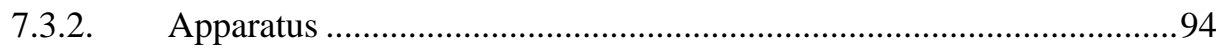

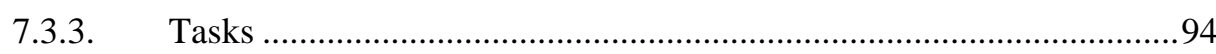

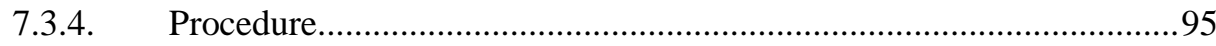

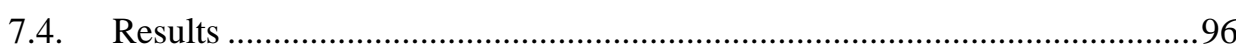

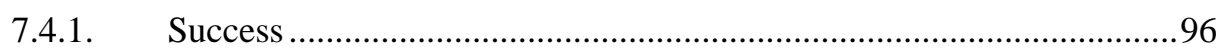

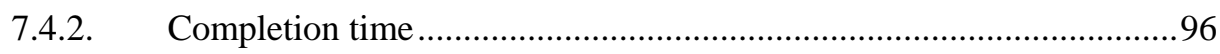

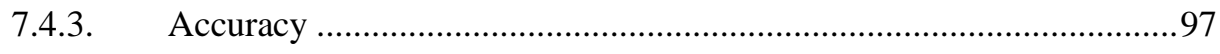

7.5. Discussion and future work ………………………...................................

8 Are Kindergarten Children Ready for Indirect Drag Interactions? ............................................................................................................... 101

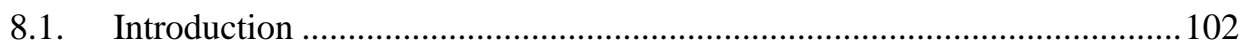

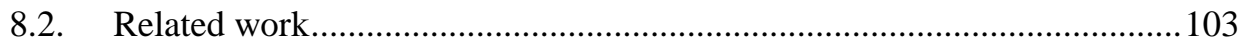

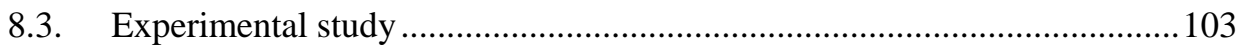

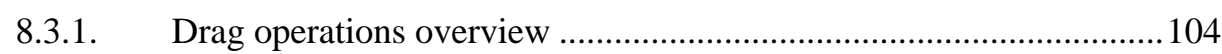

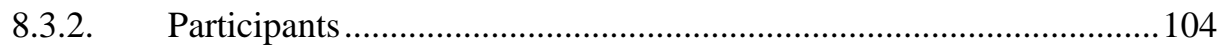

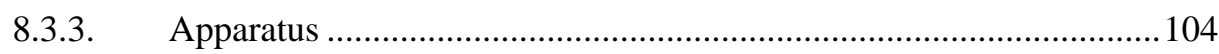

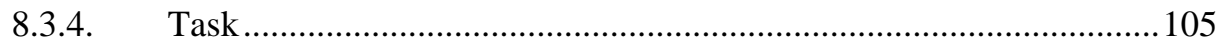

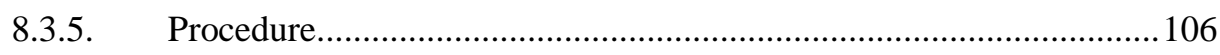

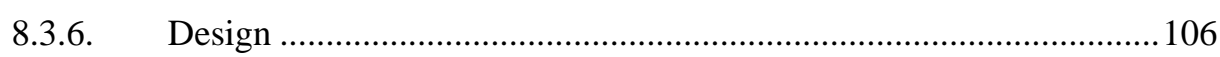

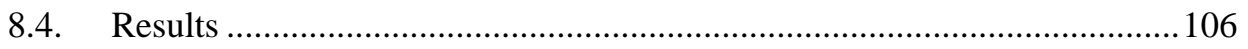




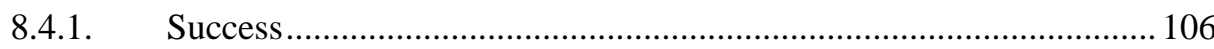

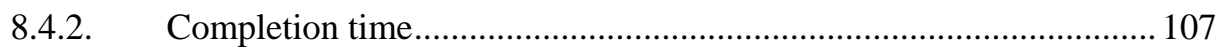

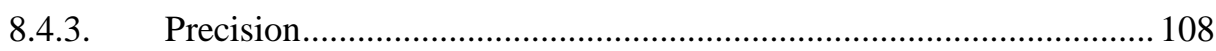

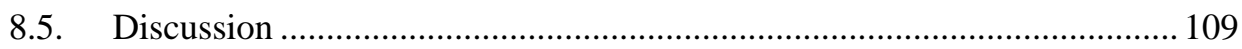

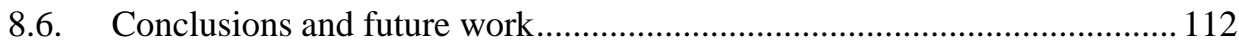

$\begin{array}{ll}\text { III Communicability } & 117\end{array}$

9 Exploring Visual Cues for Intuitive Communicability of Touch Gestures to Pre-kindergarten Children ....................................................117

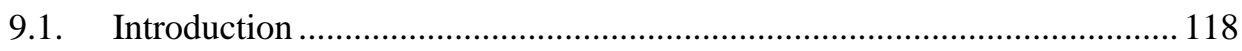

9.2. Related work ….......................................................................... 118

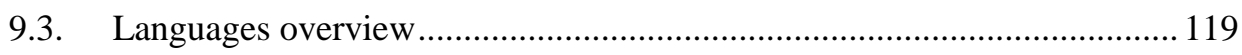

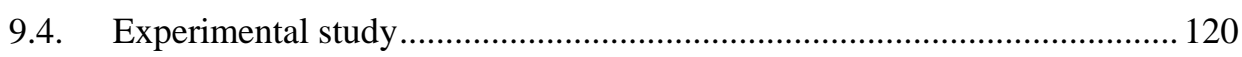

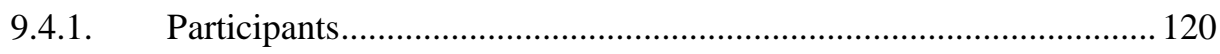

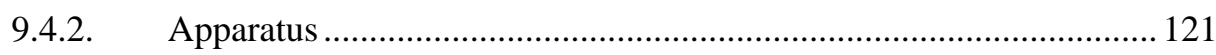

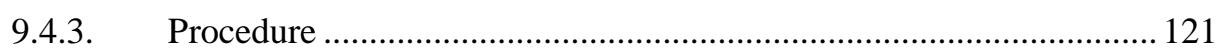

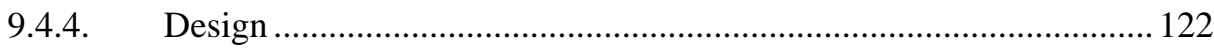

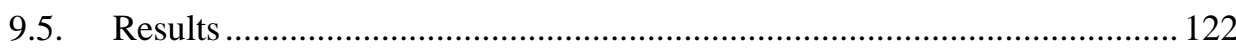

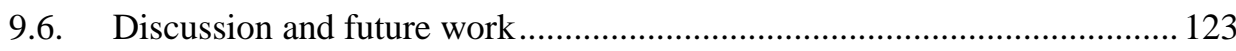

10 Evaluating Multi-touch Semiotics to Empower Pre-kindergarten Instruction with Interactive Surfaces .................................................125

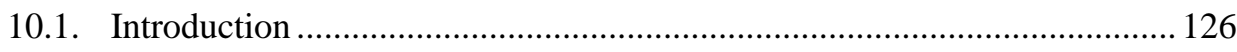

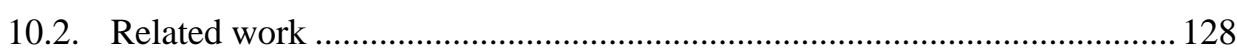

10.2.1. Industrial perspective on the communicability of multi-touch gestures 130

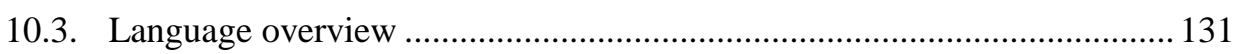

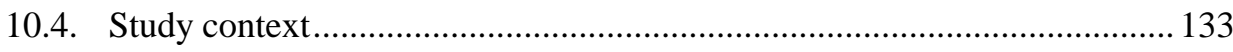

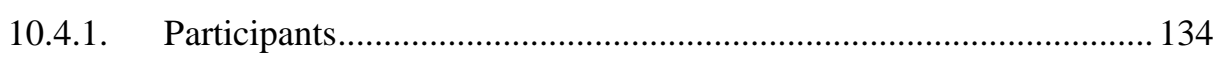

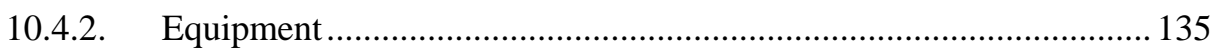


Kindertivity: Usability and Communicability Strategies for Interactive Surfaces and PreKindergarten Children

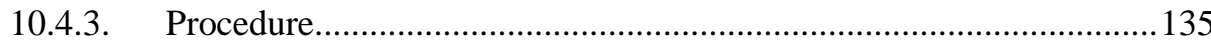

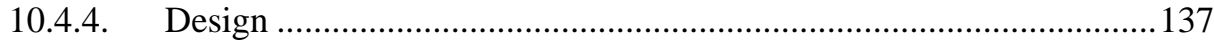

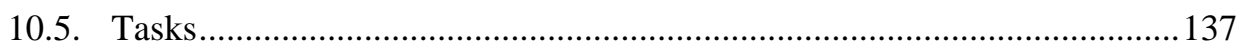

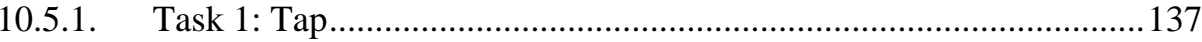

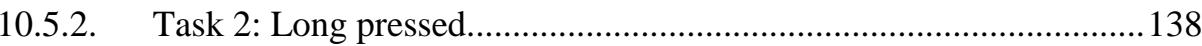

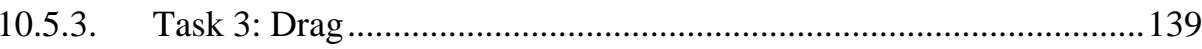

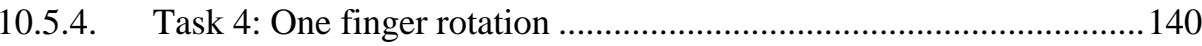

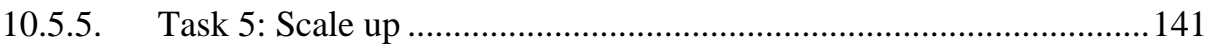

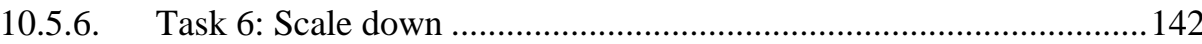

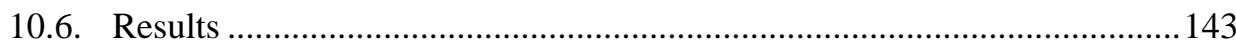

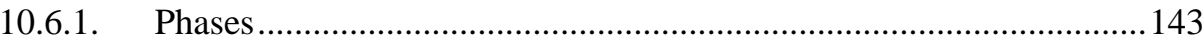

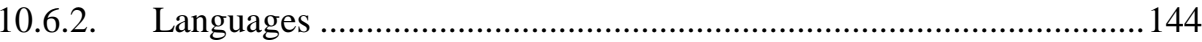

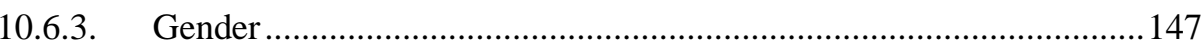

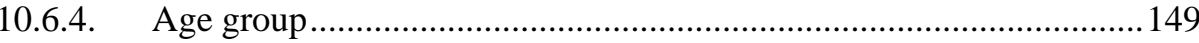

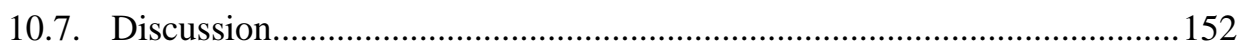

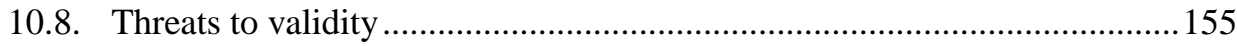

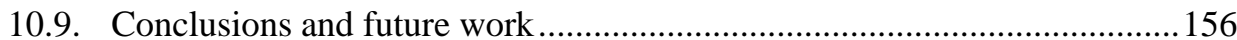

11 Exploring Visual Languages for Communicating Directional Awareness to Kindergarten Children ...................................................... 159

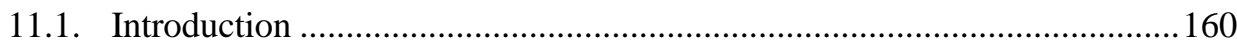

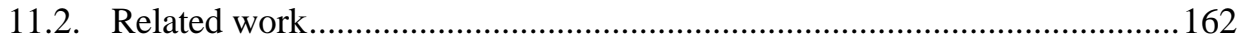

11.2.1. Industrial perspective on the communicability of spatial and directional awareness in touch devices..................................................................... 165

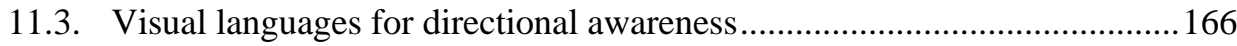

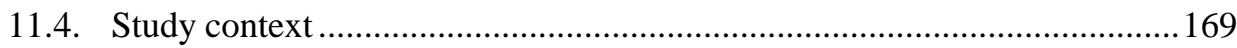

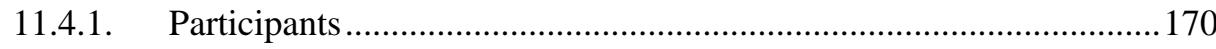

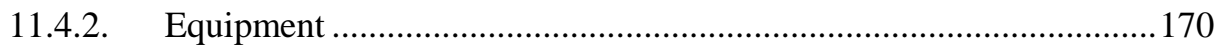

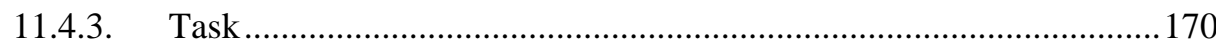

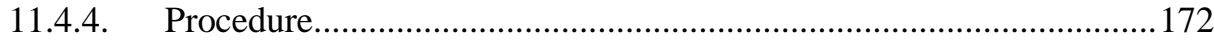

xviii 


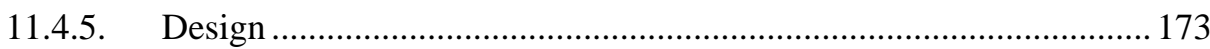

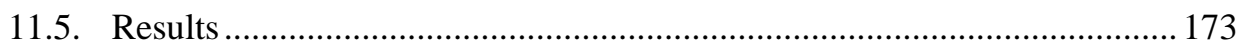

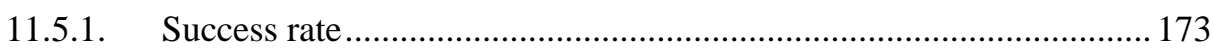

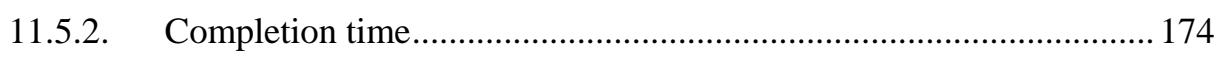

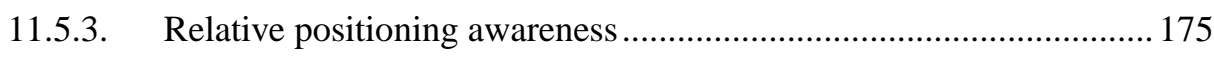

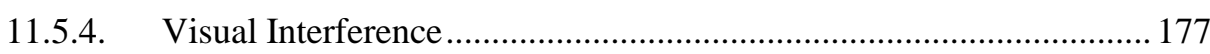

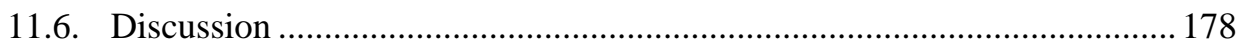

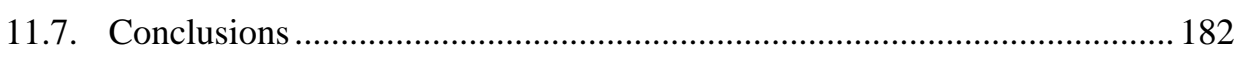

12 Evaluating Assistive Communication Languages with Kindergarten Children on Touchscreen Devices........................................................... 187

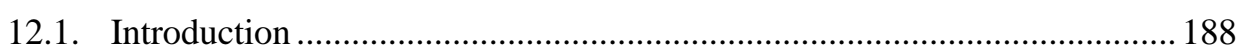

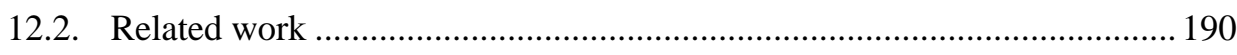

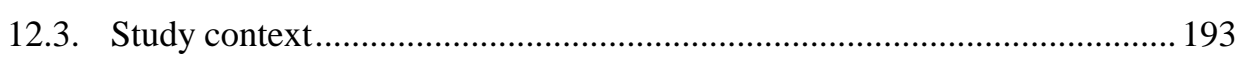

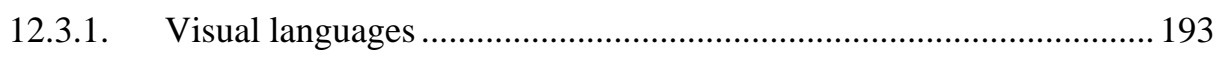

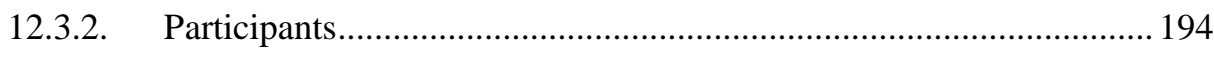

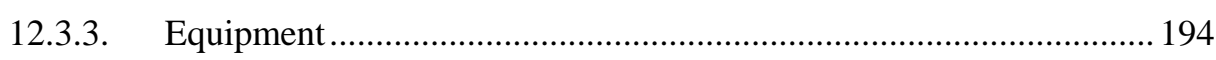

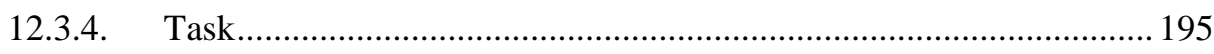

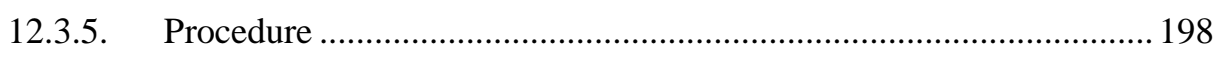

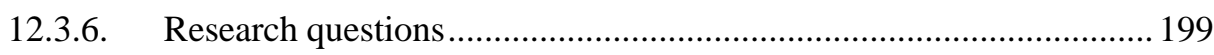

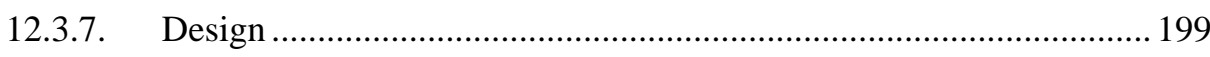

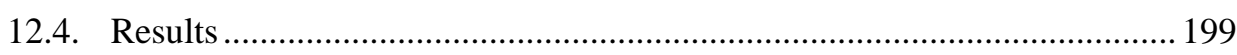

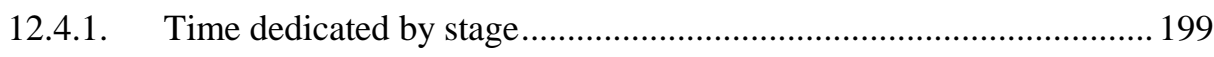

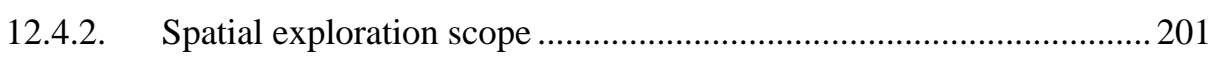

12.4.3. Gestural interaction language effectiveness ...................................... 203

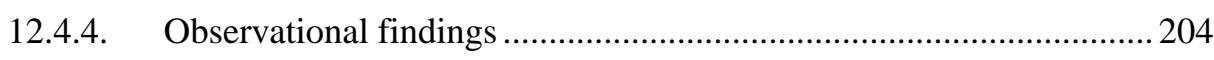

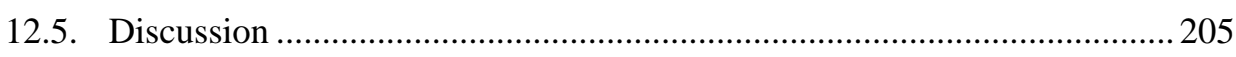

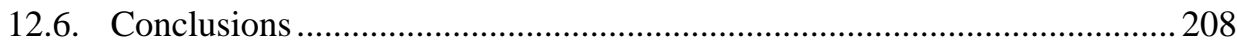


Kindertivity: Usability and Communicability Strategies for Interactive Surfaces and PreKindergarten Children

\section{$\begin{array}{ll}\text { IV Closure } & 215\end{array}$}

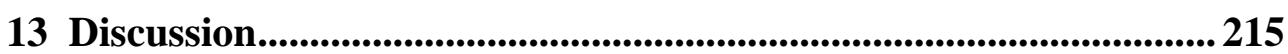

13.1. On multi-touch technology usability by kindergarten children .................216

13.2. On communicability strategies targeted to young children .....................219

14 Conclusions and Future Work ......................................................223

Bibliography ...................................................................................................... 227

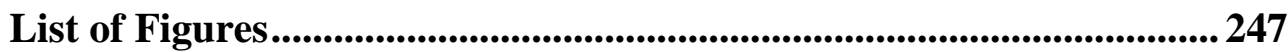

List of Tables ..................................................................................................... 253 


\section{Part I \\ Preliminaries}





\section{Chapter 1}

\section{Introduction}

\subsection{Motivation}

Since its initial steps in the eighties, even before the adoption of graphical user interfaces, to its widespread acceptance today, multi-touch technology has had huge advances (Buxton, 2013). With its growth, new sophisticated input and processing mechanisms have emerged enabling users to interact in a more natural and intuitive way (Smith, Burd, \& Rick, 2012). Multi-touch technology makes use of the direct manipulation interaction style and direct-touching to bring users more natural and intuitive interfaces. As Shneiderman and Plaisant (Shneiderman \& Plaisant, 2004) pointed out, there are three ideas behind the concept of direct manipulation: (1) visibility of objects and actions of interest; (2) replacement of typed commands by pointing-actions on objects of interest; and last but not least, (3) rapid, reversible and incremental actions that help to keep users engaged, give them control over the technology and avoid complex instructions. Additionally, there is evidence that directtouch is preferred over mediated pointing devices like the mouse as it provides a more direct way of selecting options on the screen (Hourcade, 2007). All these facts have triggered a new trend to develop applications targeted to very young children. As pointed out by Rideout (Rideout, 2011), children are real users of multi-touch technology even before they start to talk. Moreover, Rideouts' study shows that $38 \%$ of these young users have used a Smartphone, iPad or a similar device at least once and among this group, $10 \%$ were between 0 and 23 months and 39\% between 2 and 4 years old. In this line, Johnson et al. (Johnson, Adams, \& Cummins, 2012) strengthen this evidence identifying mobile devices such as smartphones and tablets as one of the two most emerging technologies suitable for young children.

Seizing the opportunity provided by the multi-touch technology, several works have assessed several dimensions in the suitability of touchscreen devices usage by children. For instance, the inherent ability of touch systems to engage users' attention from 
Kindertivity: Usability and Communicability Strategies for Interactive Surfaces and PreKindergarten Children

prescholers to adolescents has been proved (Sluis et al., 2004), (Khandelwal \& Mazalek, 2007), (Couse \& Chen, 2010). Moreover, some works have pointed out the potential of touchscreen devices to give support to scenarios in which collaboration between peers is promoted (Rick et al., 2010)(Rick \& Rogers, 2008), (Fleck et al., 2009) or creativity tasks are fostered (Helmes, Cao, Lindley, \& Sellen, 2009), (Catala, Jaen, van Dijk, \& Jordà, 2012).

However, the increasing interest in multi-touch technology has not given rise to studies on the design of multi-touch systems for the youngest age range (i.e. preschool children) yet (Hourcade, 2007). Hence, when aiming to use multi-touch screen devices to give support to kindergarteners educational activities, the technological support to be used when developing collaborative and creative skills in kindergarten children is critical since the choice of the underlying supporting technology has a great impact on the nature of the pedagogical activities to be performed in a learning context. Therefore, finding out how kindergarten children are able to interact with multi-touch screen devices becomes a critical issue.

Taking into account all the stated above, this thesis is focused on designing, developing and assessing different usability and communicability strategies for interactive surfaces when used with kindergarten children in order to establish the basis to create educational scenarios based on touchscreen devices which enrich the learning process for children in early childhood. This proposal is a step forward because of the following reasons: (1) the suitability of the interactive surfaces to support social learning since several subjects share the same physical space and, as it happens in traditional technology-free games, the communication during the creation process, experimentation and reflection is direct and not mediated by a computer. (2) The collaborative nature of the technologic infrastructure, in which users can carry out different tasks in parallel and on the same table; and (3) the creative nature of the infrastructure in which users select the game elements and the reactive behavior that it offers. The goal is that also educators have a tool on which they can design in an effective way learning activities with a technology tailored to early childhood development boosting the engagement and motivation of users in the activity.

In order to provide these strategies on the use of touchscreen devices by kindergarten children, this thesis explores multi-touch technology in four dimensions; (1) contextualization, which covers the evaluation of the types of interaction that can be performed by kindergarten children in multi-touch surfaces. (2) Assistance, which involves the definition of several assistive techniques of interaction that allow the increase of effectiveness of kindergarteners' interactions. (3) Communicability, the definition of effective mechanisms to communicate information about the application understandable to both, children and caretakers through visual languages when using the multi-touch technology. Finally, (4) adaptability to provide several strategies and 
design guidelines to allow the adaptation of the assistance and communicability mechanisms to the specific skills of each user.

\subsection{Research Hypothesis}

Relying on the elements stated in the previous section, the initial hypothesis for this thesis deals with both, the technological aspects needed to build the approach and the suitability of the solution for its use in actual scenarios based on interactive surfaces with kindergarten children. It can be expressed as follows:

"Usability and communicability strategies can be effectively designed to establish a basis for the development of applications based on touchscreen interfaces targeted to kindergarten children taking into account their development stage and abilities. This basis will provide an added value in terms of user experience, performance, engagement and quality of the educational activities and dialogues with teachers or caretakers"

\subsection{Research Methodology}

As stated above, this work attempts to provide a solution based on touchscreen devices to create appropriate scenarios targeted to kindergarten children fostering engagement and giving support to learning activities. To reach this goal, the Design Science research methodology (Hevner, March, Park, \& Ram, 2004) was applied, as it enables the design and validation of approaches to practical problems. In the words of Hevner et al., "the design-science paradigm seeks to extend the boundaries of human and organizational capabilities by creating new and innovative artifacts". The Design Science process in Information Systems firstly identifies the problem, sets the objectives of the solution, and then the solution is designed and developed, followed by an evaluation of the outcomes. In addition to applying Design Science as the main methodological approach, it was decided to adopt Wieringa's methodological proposal (Wieringa, 2009), which structures the research in nested sets of problems and tasks. As shown in Figure 1, this thesis is composed of different problem descriptions or discussions, artifact designs, evaluations, and extensions to the designs.

\subsection{Goals and Contributions}

The overall aim of this thesis is to advance in the development of suitable educational scenarios targeted to kindergarten children making use of interactive surfaces. To achieve this goal, the suitability of using touchscreen devices with kindergarten children is explored in several dimensions. First, usability issues related to the usage of touchscreen devices by children in their early developing is assessed. Then, once it is known how kindergarten children are able to interact in an effective and efficient way, the thesis explores different mechanisms to communicate information about the 
Kindertivity: Usability and Communicability Strategies for Interactive Surfaces and PreKindergarten Children

application (such as the gestures to be performed by the users to achieve the task or giving cues about where the interactive elements of the virtual world are placed). In more detail, the thesis addresses the following requirements:

- Define and evaluate a set of interaction gestures on interactive surfaces feasible to kindergarten children. This will provide with usability contextualization strategies and guidelines that assist and facilitate the use of multi-touch technology devices.

- Find out the level of accuracy that can be demanded to kindergarten children when performing multi-touch gestures.

- Provide a mechanism to move an object in a virtual 2D world tailored to children in their early development.

- Assess whether kindergarten children are able to use the virtual world beyond the screen limits.

- Define and evaluate visual languages to communicate the gestures required by the application to fulfill a specific task.

- Define and evaluate several visual languages to communicate where the interactive elements of the virtual world are located.

- Explore the impact of using different communicative visual languages simultaneously.

- Define and evaluate different assistive techniques and strategies which allow the customization of the usability and communicability techniques previously evaluated to the actual children development.

- Develop and assess a real scenario in which different usability and communicability strategies are included when the virtual world is cluttered by several interactive elements.

The contributions of the thesis are manifold: first, the definition of a basic set of multitouch gestures feasible to be performed by preschool children and the design of several indirect techniques suitable to kindergarteners; second, a set of design guidelines to help application developers to appropriately include touch interactions; third, the definition of several visual languages with different communication goals understandable by young children; fourth, a gamified application which adapts an actual educational activity by using the basis established with the usability and communicability strategies defined in this work; and, finally, a discussion of the potential use of all the strategies defined in this work to design and develop applications based on touchscreen devices which gives support to collaborative and creative processes in learning scenarios. 


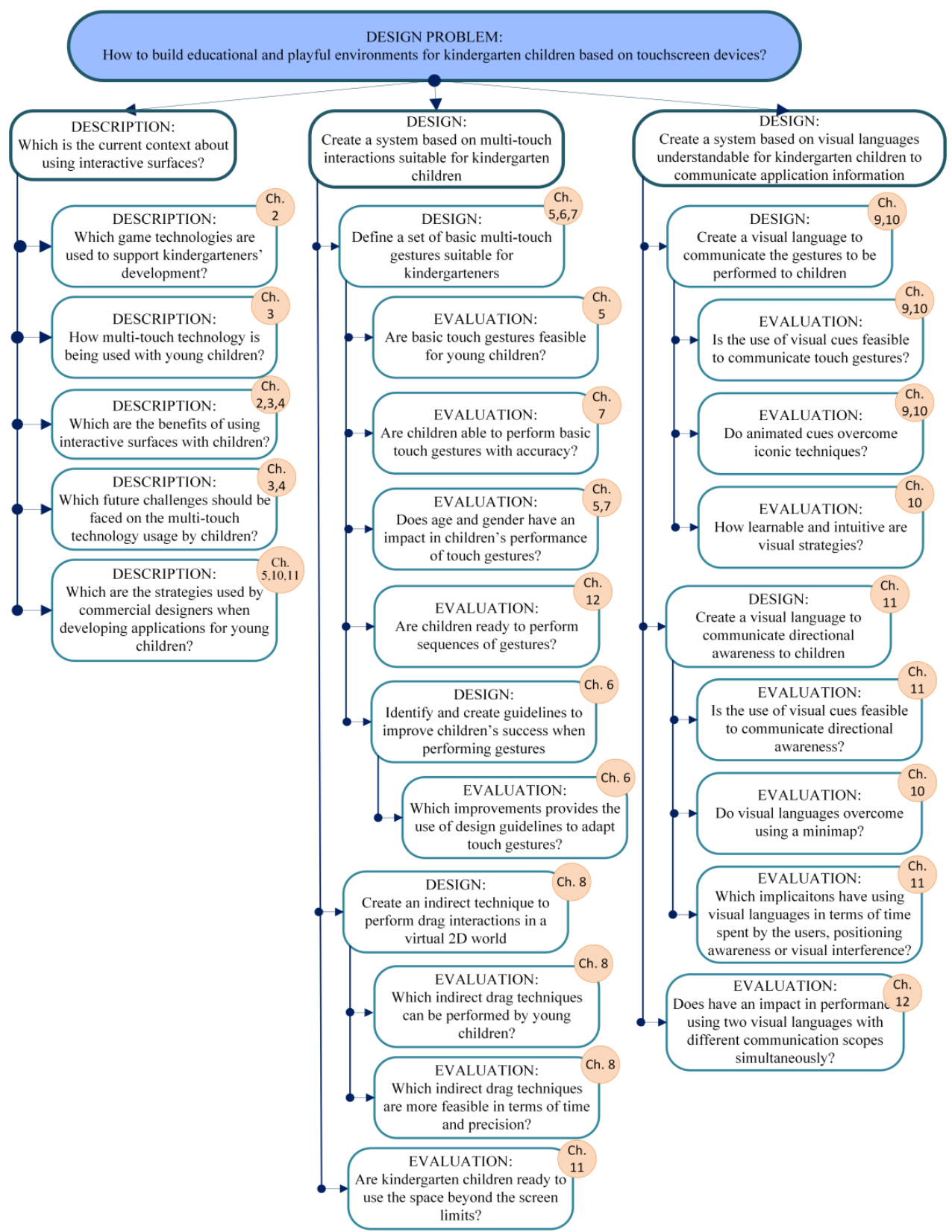

Figure 1. Problem decomposition (in rectangles) and the chapter in which they are dealt with (in circles) 
Kindertivity: Usability and Communicability Strategies for Interactive Surfaces and PreKindergarten Children

\subsection{Outline of the Thesis}

In order to explore the items described in this chapter and depicted in Figure 1, this dissertation has been divided into four parts. The first one (in which this chapter is included) serves to state the problems to be faced, to introduce the state of the art of multi-touch technology with kindergarten children and to motivate its potential use in terms of education and fostering creative collaborative activities with kindergarteners. The second part covers the contextualization work of the thesis in which the usability of different types of interaction that can be performed by pre-kindergarten children in multi-touch surfaces is evaluated. The third part explores and evaluates different communication mechanisms that allow both children and supervisors to gain awareness of different data about the applications. Moreover, this part explores the use of the usability and communicability guidelines described in the previous chapters in a real scenario. Finally, the last part of the dissertation provides a general discussion of the conducted research and draws the lines to follow in future works. A detailed description of the chapter structure is provided below:

- Part I: Preliminaries. Besides the present chapter, this part includes two additional chapters as an introduction to the field of children-computer interaction and the use of touchscreen devices. Moreover, it includes another additional chapter motivating the thesis and describing dimensions to be explored through the thesis work.

-Chapter 2. This chapter contains the publication entitled Interactive Technologies for Preschool Game-Based Instruction: Experiences and Future Challenges (Nacher, Garcia-Sanjuan, \& Jaen, 2016b). Aiming to give a general sight on the use of technology with kindergarten children, this work explores the state of the art of game technologies that support kindergarteners' development. From the analysis conducted, it was observed that different technologies such as traditional computers, interactive surfaces and robots have been previously evaluated being the last two the most emerging. However, the review shows that despite the huge growth in the number of existing educational applications based on touch devices targeted to kindergarten children, the multi-touch technology does not seem to be fully exploited for them.

-Chapter 3. This contains the publication entitled Multi-touch Technology in Early Childhood: Current Trends and Future Challenges (Nacher \& Jaen, 2015c). Once the multi-touch technology is revealed as one of the most emergent technology to design educational application targeted to kindergarteners, this paper focuses on the state of the art of the multi-touch technology and explores different works dealing with usability issues of young children when using touchscreen devices. In addition, several future 
challenges to be addressed to contribute in the Children-Computer Interaction field are identified.

-Chapter 4. This chapter contains the paper entitled KINDERTIVITY: Using Interactive Surfaces to Foster Creativity in Pre-kindergarten Children (Nacher \& Jaen, 2015b). This paper motivates the need of exploring the multi-touch technology to facilitate creative learning taking into account the cognitive and interaction limitations of very young children. Moreover, this work points out the milestones to achieve to design and develop suitable educational touchscreen scenarios.

- Part II: Usability. This part is focused on the design and evaluation of the types of interaction that can be performed by kindergarten children in multitouch surfaces. Aiming to give a starting point to design scenarios based on touchscreen devices, in this part several usability evaluations are made with users. Beginning with the study of a basic set of touch gestures based in direct manipulation, this part opens its scope and finishes testing more complex interactions such as indirect techniques. In addition, several assistive techniques of interaction which allow the increase of effectiveness of kindergarteners' actions are defined.

-Chapter 5. This chapter contains the publication entitled Multi-touch gestures for pre-kindergarten children (Nacher, Jaen, Navarro, Catala, \& González, 2015). This paper provides several contributions. The first is a study of the commercial perspective of the multi-touch technology targeted to kindergarten children showing that most existing educational applications for these users do not take fully advance of the technology. The second contribution is the experimental confirmation that young children are able to perform a basic set of multi-touch gestures. In addition, cognitive and precision issues when children perform these gestures are identified and corresponding design guidelines are proposed to minimize their impact on the performance and usability in the interaction.

-Chapter 6. This chapter contains the paper entitled Improving Pre-Kindergarten Touch Performance (Nacher, Jaen, Catala, Navarro, \& Gonzalez, 2014). This work evaluates the specific strategies defined in the previous chapter to deal with the children issues when performing the double tap and long press gestures. From the results of this work it can be seen that the guidelines implementations have a positive effect on success rates of these two gestures, being feasible their inclusion in touchscreen applications targeted at children in early childhood.

-Chapter 7. This chapter contains the work entitled Evaluating the Accuracy of Pre-Kindergarten Children Multi-touch Interaction (Nacher \& Jaen, 2015a). Once it has been proved that kindergarten children are able to 
Kindertivity: Usability and Communicability Strategies for Interactive Surfaces and PreKindergarten Children

perform basic multi-touch gestures with high success rates, this work evaluates children abilities to perform gestures that require movement of contacts in the surface when accuracy is required in the termination phase of these gestures. Children abilities are revealed from the evaluation and assistive strategies that adapt their behavior to the actual levels of motor and cognitive development of each child are proposed.

-Chapter 8. This chapter contains the work Are Kindergarten Children Ready for Indirect Drag Interactions? (Nacher, Ferreira, Jaen, \& GarciaSanjuan, 2016), which proposes and evaluates different techniques to be used when the direct manipulation style is not feasible due to application requirements. Three indirect drag techniques are evaluated and their weaknesses and strengths are described.

- Part III Communicability. This part of the dissertation focuses on the definition of effective mechanisms to communicate information about the application requirements and context through the use of visual languages understandable to both children and supervisors. Languages to communicate two types of data are explored in order to provide an effective mechanism that reduces the number of technical scaffolding and fosters dialogues related to the learning activity. The first two chapters present and assess languages to communicate data about the gestures to be performed while the last one explores and evaluates visual languages to provide directional awareness.

-Chapter 9. This contains the paper Exploring Visual Cues for Intuitive Communicability of Touch Gestures to Pre-kindergarten Children (Nacher, Jaen, \& Catala, 2014). This work provides preliminary insights in the field of using visual languages to communicate the expected touch interactions to kindergarteners and caretakers. The evaluation reveals that using animated visual languages may be an appropriate approach to communicate the desired interactions to young users.

-Chapter 10. This chapter contains the publication Evaluating multi-touch semiotics to empower pre-kindergarten instruction with interactive surfaces (Nacher, Jaen, \& Catala, 2017). This paper extends the work done in the previous chapter by including a wider and comprehensive range of basic touch gestures, including an evaluation of the languages' learnability and memorability and including a third visual animated language avoiding the explicit use of a hand to communicate. Finally, based on the evaluation, the best approaches are pointed out to effectively communicate touch gestures to kindergarten children by using visual cues.

-Chapter 11. This chapter contains the paper Exploring visual languages for communicating directional awareness to kindergarten children 
(Nacher, Jurdi, Jaen, \& Garcia-Sanjuan, 2018). This work focusses on the design and evaluation of different approaches to communicate directional awareness to kindergarten children. Three different visual languages are designed and evaluated providing a comprehensive description of the efficacy and effectiveness of each approach in terms several factors such as success rate, time spent by the users, positioning awareness and visual interference. Moreover, this work assesses the suitability of using a virtual world beyond the screen which is being underused in actual commercial applications targeted to kindergarteners.

-Chapter 12. This chapter contains the publication entitled Evaluating assistive communication languages with kindergarten children on touchscreen devices (Nacher, Garcia-Sanjuan, \& Jaen, 2018). This work makes use of all the design guidelines and communication languages explored in the previous chapters in order to assess whether their simultaneous use in an actual educational application is feasible when working with children in their early childhood. In this work, an actual application targeted to hospitalized children is gamified and adapted to kindergarteners' development and abilities. The adapted design includes most of the guides proposed in the previous chapters such as the use of different multi-touch gestures, the use of the space beyond the screen limits, the inclusion of two different visual languages to communicate data, etc. This work is as an exemplification of how the work carried out in the previous publications serves as a basis and a starting point to design and develop appropriate educational collaborative scenarios targeted and tailored to kindergarten children by using interactive surfaces.

- Part IV: Closure. This part of the thesis includes a discussion of the several contributions and aspects addressed in this dissertation, as well as it highlights the lines to follow in the future to design educational scenarios targeted to kindergarten children using touchscreen devices.

To sum up, the work described in the thesis has produced 11 research papers: 6 read at international conferences (4 CORE A, 2 international conferences), 3 published in international scientific journals (1 JCR Q1, 1 JCR Q3) and 2 JCR scientific journals which are still under review. 



\section{Chapter 2}

\section{Interactive}

Technologies for

Preschool Game-Based

Instruction:

\section{Experiences and Future Challenges}

Vicente Nacher, Fernando Garcia-Sanjuan, Javier Jaen

ISSI Group, Dpto. Sistemas Informáticos y Computación, Universitat Politècnica de València.

Published in Entertainment Computing 17, pp. 19-29. Elsevier (2016) 
Kindertivity: Usability and Communicability Strategies for Interactive Surfaces and PreKindergarten Children

\begin{abstract}
According to current kindergarten curricula, game play is an important basis for children development and it is the main driving force when designing educational activities during early childhood. This paper presents a review of the current state of the art of game technologies that support pre-kindergarten and kindergarten children development. Moreover, the most emergent technologies for developing educational games for preschool children are identified and a set of future challenges are discussed. The main goal of this work is to review the state of the art in interactive technologies which will help educators, game designers and Human-Computer Interaction (HCI) experts in the area of game-based kindergarten instruction.
\end{abstract}

\title{
2.1. Introduction
}

According to Huizinga, play is innate to human culture (Huizinga, 1985) and children play in many ways and with different types of artifacts (Fein, 1981). The importance and benefits of game play in early childhood education and development are manifold as discussed by Singer et al. (Singer, Golinkoff, \& Hirsh-Pasek, 2006) who studied how play motivates and enhances children's cognitive and social-emotional growth, and by Ginsburg (Ginsburg, 2007) who pointed out that play is essential for development contributing to the cognitive, physical, social and emotional well-being. Consequently, play should be a basic pillar in children education and development as pointed out by Plato (Plato, 1955): "Enforced learning will not stay in mind. So avoid compulsion and let your children's lessons take the form of play".

However, despite the large number of works addressing children play (e.g., (Barnett, 1990)(Samuelsson \& Carlsson, 2008), (Morrow, 1990), (Frost, Wortham, \& Reifel, 2011), (Veitch, Bagley, Ball, \& Salmon, 2006)(Parten, 1933)) and the presence of games in children educational curricula, considerable less research has focused on the relationship between play, learning and technology in the context of early childhood education. In this respect, traditional desktop computers have often been considered as the main technological devices to be used, leaving new emerging technologies such as interactive surfaces and robots, which could be exploited to obtain richer game experiences, underused.

Therefore, in this paper we provide a review of works that use technologies to develop games to help preschool children improve the three dimensions of their development mentioned above: physical, socio-affective and intellectual. The analysis carried out shows that despite there are technologies with suitable mechanisms to support very young children instruction based on play, there are still missing aspects that need to be addressed in order to fully support these dimensions in children's development. Hence, we provide a set of future areas of work that can be addressed in the near future. The 
end goal is to define a research path to give educators and designers appropriate guidelines to design game activities using each technology and to devise games and educational activities that smoothly combine all the evaluated technologies creating environments that foster preschool children's development.

\subsection{Technology-Supported Games for Preschool Children}

In the literature, many previous works using technology-aided learning activities to support preschool children (aged 2-6 years) development can be found. In this section, these works are explored and classified by the main technological components they rely on, namely traditional computers, interactive surfaces and also robots and other technologically-enhanced toys. Their different interaction mechanisms are discussed, as well as the affordances they present and their potential to design games that stimulate preschooler's physical, socio-affective and intellectual abilities. The works' selection process involved the analysis of the main conferences and journals in the field of HCI looking for technology evaluations with pre-kindergarten and kindergarten children. Then, the obtained papers were classified in terms of the technology used. Moreover, a representative set of studies/games in each category were selected.

\subsubsection{Traditional Computers}

In the past two decades, the main strand when developing intellectual and cognitive abilities among kindergarten children through technology was the use of traditional computers, which were mainly interacted via mouse and keyboard. Since regular keyboards have too many keys and can cause difficulties for very young children to interact with, some approaches required either disabling or removing some keys in order to simplify interactions. Jones and Liu (Jones \& Liu, 1997), for instance, evaluated how 2 to 3 year-old children interact with a computer. For this purpose, they designed a videogame which used visual stimuli, animations and audio to capture the kid's attention. The computer told the child to press a certain keyboard button, and informed the user whether the interaction had been successful. Simplification was achieved in this case by using only a few keys of the keyboard, disabling the rest. The game contained educative contents in order to enhance vocabulary through learning colors, toy names, food, computer parts, etc., and also to learn mathematical concepts such as big/small, or logical relations like cause/effect (e.g., if a key is pressed, something will happen on the screen). In their study, the researchers observed that meaningful interactions with this kind of technology do not appear before 2.5 years of age, but perhaps their findings were biased by having too many keys presented to the participants, even though not all of them were responsive. Another example is the work by van Daal and Reitsma (van Daal \& Reitsma, 2000) which evaluated a computer assisted program for learning to read and spell. The program includes several exercises such as matching pictures with spoken words, indicating which letter sound is heard and matching written words with pictures and spelling a word by its sound (see Figure 
Kindertivity: Usability and Communicability Strategies for Interactive Surfaces and PreKindergarten Children

2). The results showed that the children who used the computer-based reading and spelling activities learned to name more letters and they were able to read more words than the group of children who did not have access to the computer-based practice. Hence, the proposed approach helped children in the improvement of their recognition and decoding skills.

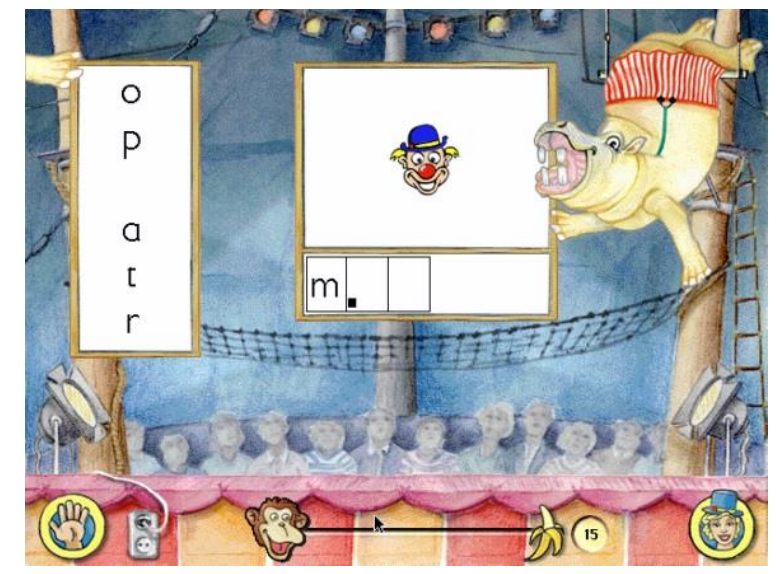

Figure 2. Example screen of the activity spelling a word by its sound (from (van Daal \& Reitsma, 2000)).

Instead, other works get rid of keyboards completely and replace them by other intrinsically simpler devices. This is the case of Strommen et al. (Strommen, Revelle, Medoff, \& Razavi, 1996), who designed a videogame that consisted on directing a Cookie Monster through a path up to a given target cookie for him to eat. They designed the game to be controlled by a mouse, joystick or trackball, and performed an evaluation to assess which input device improved precision tasks on 3-year-olds. The results showed the trackball was the more accurate, but the slowest, way to interact. Similarly, Ahlström and Hitz (Ahlström \& Hitz, 2013) evaluated precise pointing interactions using mouse on children aged 48-58 months. In order to do so, they proposed a game that consisted on selecting and dragging colored elements on the screen. Results showed that an assistive technique can improve children's pointing accuracy. Navarro-Newball et al. (Navarro-Newball et al., 2014) reduced the complexity of the interaction even further. They shied away from an interaction mediated by keyboard and mouse and designed a videogame which required speech interaction and gave visual feedback to children. The game, Talking to Teo (see Figure 3 ), was aimed at the rehabilitation of children with early diagnosed hearing impairment. During the experiments carried out with a therapist it was evidenced that the game resulted enjoyable and engaging for children when performing a task usually boring for them such as repetitive speech mechanization sessions. 
Despite devising easier interaction mechanisms for children, scenarios with this type of technology are mainly mono-user, where, if several users are present in front of a computer, one tends to retain the control whereas the rest adopt a more passive (observer) role. This could complicate the design of games to foster social abilities. Besides, experiences are limited to happen in front of the computer, because these are fixed to a single location. Therefore, it would be difficult for kids to engage in games that encouraged mobility and physical exercise using this kind of technologies. However, other types of physical development, such as the improvement of fine motor skills, could be trained using traditional computers. As an example, the Cookie Monster game by Strommen et al. (Strommen et al., 1996) and the drag game by Ahlström and Hitz (Ahlström \& Hitz, 2013) require precision in order to succeed. Even though these two works were not aimed at training any specific capacity, in our opinion, videogames that require this type of precision could be used to improve fine motor skills on children.
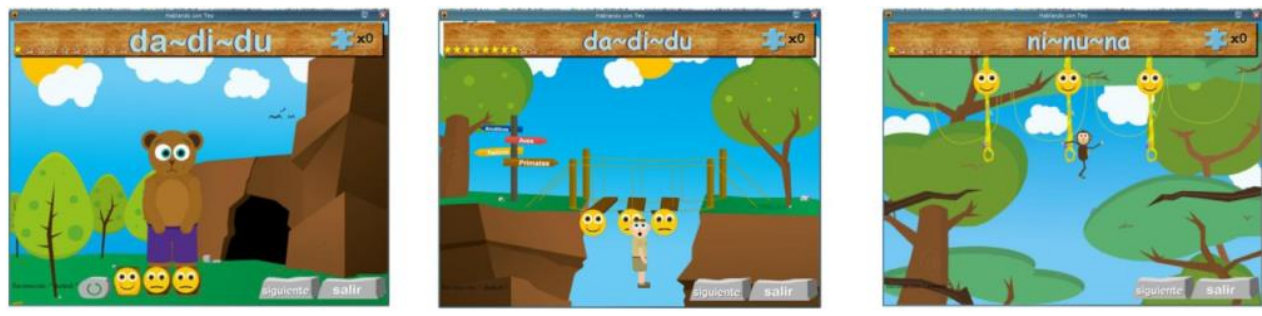

Figure 3. Mini-games examples of Talking to Teo game (from (Navarro-Newball et al., 2014)).

\subsubsection{Interactive Surfaces}

The natural and intuitive way of interaction provided by the multi-touch technology (Smith et al., 2012) makes it ideal for preschool children. The three basic ideas behind the direct manipulation style that enables a natural interaction are stated by Shneiderman et al. (Shneiderman, Plaisant, Cohen, \& Jacobs, 2009): the first one is the visibility of objects and actions of interest; the second one, the replacement of typed commands by pointing-actions on the objects of interest; and, finally, the rapid, reversible and incremental actions help children to keep engaged and give them control over the technology avoiding complex instructions that complicate the interaction. In recent years, several studies have focused on the use of multi-touch interactive tabletops with educational purposes, not only because of the naturalness of the interactions but also because they allow co-located experiences where social skills such as collaboration can be fostered. For example, Kammer et al. (Kammer, Dang, Steinhauf, \& Groh, 2014) presented three applications to foster the development of cognitive and motor skills on a tabletop with children aged 4 to 6 years. The experiment conducted showed that preschool children were able to use this technology and they enjoyed the task and collaborated in the multi-user activity. Tyng et al. (Tyng, Zaman, \& Ahmad, 2011a) developed a set of applications for teaching preschool 
Kindertivity: Usability and Communicability Strategies for Interactive Surfaces and PreKindergarten Children

mathematics also based on a multi-touch tabletop (see Figure 4-a). Their results showed that despite the difficulty of children understanding the concepts of addition and subtraction operations, the platform helped children learn them and the interactive environment facilitated collaboration and engagement. Another interesting example is the work of Mansor et al. (Mansor, De Angeli, \& de Bruijn, 2009) who conducted a comparison of a physical setting versus a collaborative multi-touch tabletop setting with children aged between 3 and 4 years and suggested that kids should remain standing during these operations because, otherwise, they would find it difficult to drag objects on the surface due to bad postures. Finally, Huber et al. (Huber et al., 2016) evaluated the transfer of learning from a touchscreen device to the real world in children aged 4 to 6 years. The kids performed two tasks: first they learned how to solve a problem, consisting on solving a Tower of Hanoi, using a touchscreen device; then, they had to solve the same problem with physical objects. The results showed that children got significant improvements from the practice irrespective of the type of manipulation performed (physical objects vs touchscreen device). Hence, the authors concluded that children's learning on the touchscreen device was appropriately transferred to the physical version.

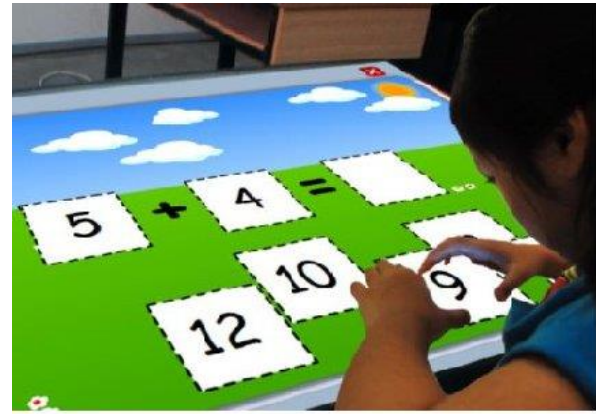

(a)

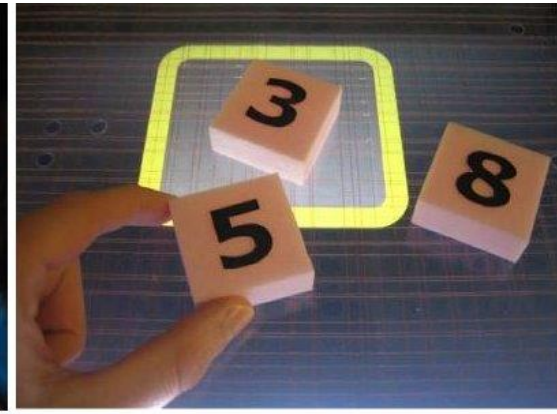

(b)

Figure 4. (a) MEL-Vis from (Tyng et al., 2011a). (b) The Teaching Table from (Khandelwal \& Mazalek, 2007).

Multi-touch interaction on digital tabletops can be extended with the use of tangibles, which provide a more natural and intuitive means of interaction. For example, Yu et al. (Tyng, Zaman, \& Ahmad, 2011b) presented a set of applications for children aged between 5 and 6 years. These applications contributed to the development of intelligence and linguistic, logical, mathematical, musical and visual-spatial capabilities through activities such as listening to a word and picking out the picture that represented it, shooting balloons with the right numbers, etc. Following the same research path, Khandelwal \& Mazalek (Khandelwal \& Mazalek, 2007) presented the Teaching Table system based on a tabletop for basic mathematics learning for prekindergarten children (see Figure 4-b). This work showed that children aged between 3 
and 6 years were engaged with the platform and it allowed collaborative actions when learning through play. Further, Sluis et al. (Sluis et al., 2004) designed Read-It, a multimodal, tangible and collaborative tabletop application that was shown to effectively support children aged 5 to 7 to read.

Finally, Marco et al. (Marco, Cerezo, Baldasarri, Mazzone, \& Read, 2009; Marco, Cerezo, \& Baldassarri, 2009) designed the Farm Game (see Figure 5); a virtual farm with a farmer as a virtual agent shown on the screen. The interaction is carried out by manipulating physical toys over the table surface and a visual recognition software translates the real condition into a 3D virtual environment shown on a TV. The authors pointed out that the platform could be used to implement a storytelling game and, additionally, adding more complex behavior with emotional content could help to a richer playing experience.

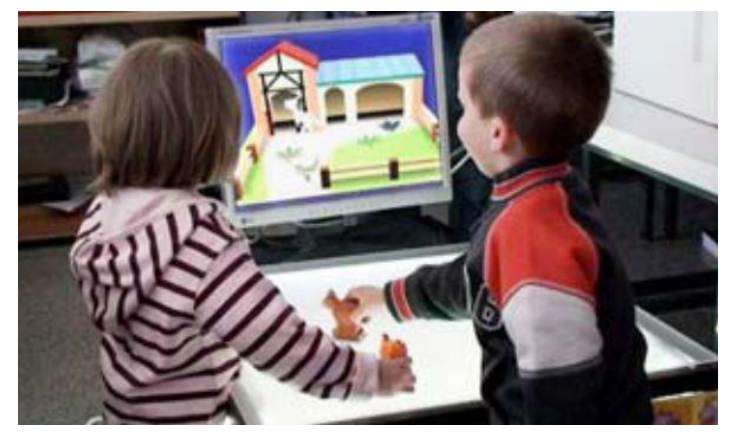

Figure 5. Children playing in the The Farm Game (Marco, Cerezo, Baldasarri, et al., 2009; Marco, Cerezo, \& Baldassarri, 2009).

\subsubsection{Tablets and smartphones}

In a similar way to computers, one drawback of tabletops is their reduced mobility, hence not being very suitable to design games that might involve physical exercise. Recent research (Rädle, Jetter, Marquardt, Reiterer, \& Rogers, 2014) (Garcia-Sanjuan, Jaen, Catala, \& Fitzpatrick, 2015) has shown that tablets and smartphones, which are nearly of common use nowadays, can be placed together on a flat surface and form a multi-display environment that resembles a tabletop. Therefore, multiple children can engage together in the same game in a co-located way where social skills can be developed and these handheld devices can also be moved in order to add physical mobility to the games.

Many sources have praised the suitability of tablets and smartphones for being used in education with very young children, In this respect, the Horizon report (Johnson et al., 2012) placed them as emerging technologies suitable for children aged under 2 years, and Hirsh-Pasek et al. (Hirsh-Pasek et al., 2015) pointed out that the most popular category in the Apple App Store is the "educational" category made of more than 80,000 applications. Indeed, several studies have recently delved into using tablets and 
Kindertivity: Usability and Communicability Strategies for Interactive Surfaces and PreKindergarten Children

smartphones in preschool instruction. Zaranis et al. (Zaranis, Kalogiannakis, \& Papadakis, 2013) conducted an experiment to evaluate the effectiveness of digital activities on smart mobile devices (tablets) when teaching mathematical concepts to kindergarten children such as general knowledge of numbers, efficient counting, sorting and matching. Their results confirmed that the tablet-aided learning provided better learning outcomes than the traditional (non-technological) teaching method. Another study conducted by Chiong and Shuler (Chiong \& Shuler, 2010) involved audiovisual material on touch devices adapted to children aged 3 to 7 years and their results showed that children obtained remarkable gains in vocabulary and phonological awareness. Another work using tablets is the study by Berggren and Hedler (Berggren $\&$ Hedler, 2014) in which the authors presented CamQuest, a tablet application that enables children to move around and recognize geometric shapes in the real objects that they see. The tablet shows the images from the camera and the application integrates the geometric shape to look for (see Figure 6). This application combines the learning of shapes (such as circle, square, rectangle and triangle) with active play since children are investigating their surroundings. Moreover, the application can be used in pairs therefore fostering collaboration, and enables defining roles between participants, so that children develop their social skills.

Many other studies have focused on the feasibility of multi-touch interactions on these small devices for such group of users. For instance, the works by Nacher et al. (Nacher et al., 2015), (Nacher, Jaen, Catala, et al., 2014), not only reveals the huge growth in the number of existing educational applications targeted to pre-kindergarten children, but also evaluates a set of basic multi-touch gestures (tap, double tap, long pressed tap, drag, scale up, scale down, one finger rotation and two finger rotation) in a tablet with children aged between 2 and 3. Their results showed that pre-kindergarten children are able to perform successfully the tap, drag, scale up, scale down and one-finger rotation gestures without assistance, as well as the long pressed and double tap gestures with some assistive techniques that fit the gesture to the actual abilities of children. Another interesting study was conducted by Vatavu et al. (Vatavu, Cramariuc, \& Schipor, 2015) who evaluated the tap, double tap, single hand drag and double hand drag gestures (see Figure 7) with children between 3 and 6 years with tablets and smartphones. Overall, their results showed good performance except for the double hand drag gestures, which were affected by some usability issues. Moreover, their results showed a correlation between children with higher visuospatial skills (i.e. having better skills for understanding relationships between objects, as location and directionality) and a better performance in the drag and drop tasks and the accuracy when performing tap gestures. The work by Nacher and Jaen (Nacher \& Jaen, 2015a) goes a step further and presents a usability study of touch gestures that imply movement of the fingers on the tablet (drag, scale up, scale down and one finger rotation) requiring high levels of accuracy in the termination phase of the gestures. Their results showed that very young children (from 2 to 3 years old) are able to perform these gestures but with significant 
differences between them in terms of precision depending on their age since they are in the process of developing their fine motor skills. Finally, the authors proposed as a future work an adaptive mechanism that fits the required accuracy to the actual level of development of each child.

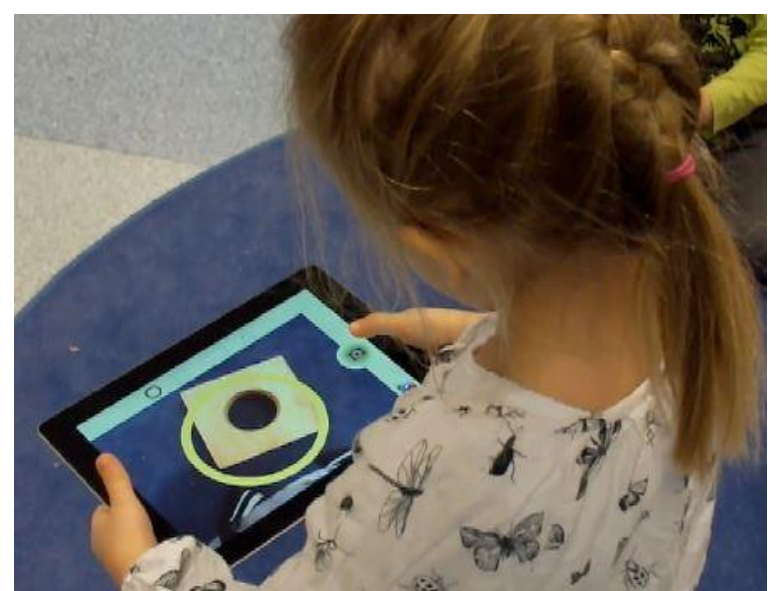

Figure 6. Child interacting with CamQuest (extracted from (Berggren \& Hedler, 2014)).

Nacher et al. (Nacher, Jaen, \& Catala, 2014) (Nacher et al., 2017) also made an analysis of communicability of touch gestures comparing three visual semiotic languages (see Figure 8). The results showed that the animated approaches had higher success rates and they overcame the iconic one. The results also showed that the animated approaches had better success rates with dynamic gestures (i.e. those requiring movements of contacts on the surface) reaching a $90 \%$ of success in gestures such as drag, rotation, scale up and scale down. Hence, basic reasoning related to the interpretation of moving elements on a surface can be effectively performed during early childhood. Moreover, since the results did not showed differences between the two animated languages, the explicit representation of a hand is not a mandatory element for communicability with prekindergarten children. The work by Hiniker et al. (Hiniker et al., 2015) evaluated different types of prompts for eliciting gestures such as double tap, horizontal and vertical swipe and shaking the tablet. They evaluated prompts such as in-app audio, on-screen demonstrations (with hand demos or changing the visual state of the item) and instructions by an adult model with children aged between 2 and 5. Their results showed that despite the most effective one was the adult guidance, children aged 3 years or older were able to follow other types of cues. These languages or prompts could help children identify direct mappings between visual stimulus and their associated touch gestures. Therefore, the use of these languages could be particularly interesting in the development of games in which preschool children could play autonomously. Moreover, the use of these semiotics might help children in developing their symbolic thinking. Although the studies listed above 
Kindertivity: Usability and Communicability Strategies for Interactive Surfaces and PreKindergarten Children

developed their applications for experimental purposes, these or similar applications could be used as games in order to help children in their fine-motor and visuospatial skills development through interactive surfaces.
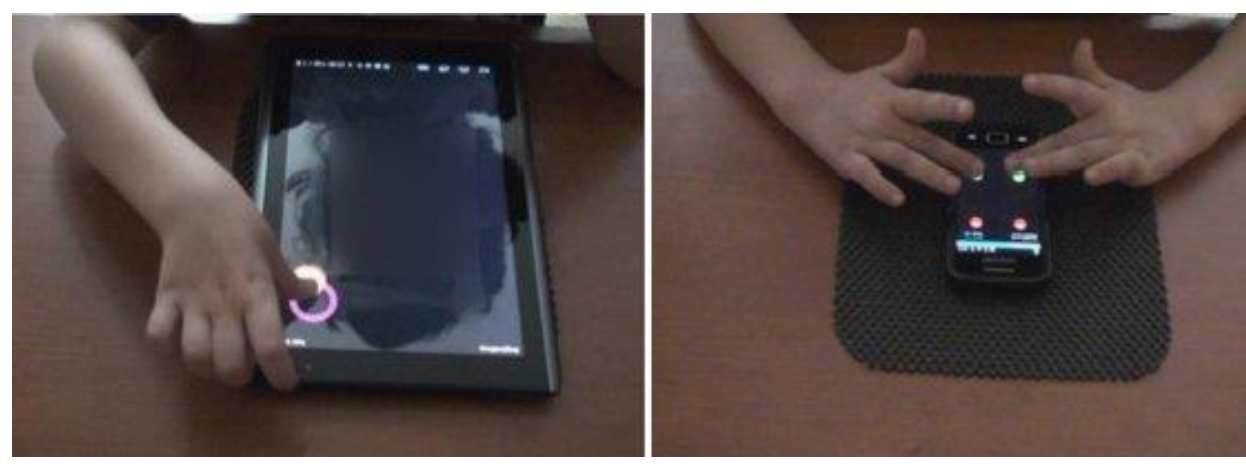

Figure 7. Child performing simple and double drag gestures (extracted from (Vatavu et al., 2015)).

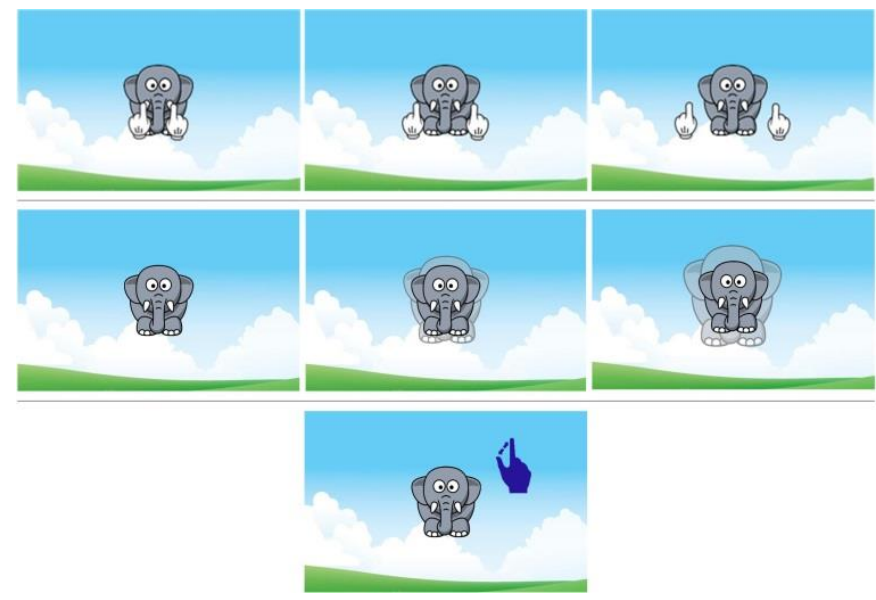

Figure 8. Description of the animated hand (top), the animated object (middle) and the iconic (bottom) language for the scale up gesture (extracted from (Nacher et al., 2017)).

\subsubsection{Robots and Technologically-Enhanced Toys}

Unlike computers or interactive surfaces, tridimensional toys and robots have the capacity of being grasped, hence serving as a sort of tangible user interface (TUI), which presents an added value in childhood education "as they resonate with traditional learning manipulatives" (Strawhacker \& Bers, 2014). The research activities to design robots for (pre-)kindergarten children have focused on developing intellectual 
capacities such as linguistic aptitudes. In this respect, Ghosh and Tanaka (Ghosh \& Tanaka, 2011) designed a Care-Receiving Robot (CRR) to give support in English learning to children aged between 3 and 6 years. Two games were proposed: a game to learn colors and another to learn vocabulary about animals. In the first one, called Color Project, the kids showed a colored ball to the robot and told it which color it was. Then, the robot touched the ball and guessed its color. In the second game, Vocabulary Project, a series of flashcards were shown to the robot, and it had to guess which animals they represented. The methodology used implied that children acted as teachers and the robot adopted the role of the pupil. In both games, the kid had to correct the robot when it was wrong, or to congratulate it when it answered correctly. The results of the experimentation with children revealed that they were very motivated at first, but tended to feel bored and frustrated quickly if the robot was too often right or wrong, respectively, because the game became monotonous. Tanaka and Matsuzoe (Tanaka \& Matsuzoe, 2012) went further and revealed that kids aged 3 to 6 are capable of learning verbs by playing with the $C R R$, and they even suggested that learning through playing with the robot might be more effective than not involving such a tangible artifact. Soute and Nijmeijer (Soute \& Nijmeijer, 2014) also designed a robot aiming to help in the language and literacy skills development. In this case, an owlshaped robot (see Figure 9-a) to perform story-telling games with children aged 4 to 6 was designed. The robot narrated partial stories that children had to complete by showing flashcards to it. The small study session conducted showed that the system was engaging for the kids.

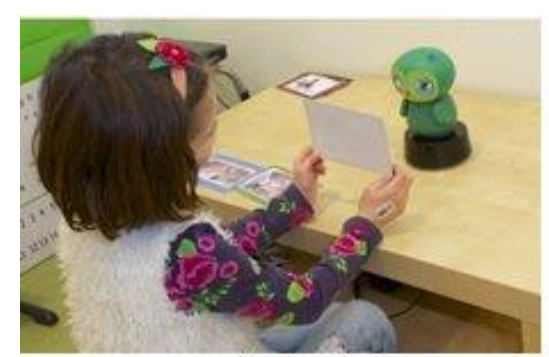

(a)

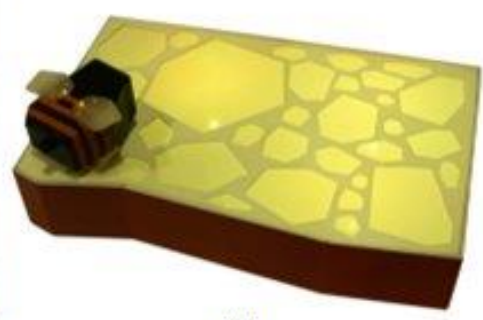

(b)

Figure 9. (a) Owl-shaped robot (extracted from (Soute \& Nijmeijer, 2014)). (b) Beelight (extracted from (Shen et al., 2013)).

Shen et al. presented Beelight (Shen, Qiu, Li, \& Liu, 2013), a system composed of a bee-shaped robot and a tabletop serving as its honeycomb (see Figure 9-b), aimed at teaching colors to children aged 4 to 6 years. Two games were implemented with this approach. On the one hand, Color Sharing, in which the kids would grab the robot and show a color to it. Then, the bee would glow in said color and, if placed on the honeycomb, it would be colored as well. The second game, Color Searching, would consist of the bee being illuminated with a given color and the children having to 
Kindertivity: Usability and Communicability Strategies for Interactive Surfaces and PreKindergarten Children

search for some object of said color and place it on the honeycomb. In case of success, the honeycomb would play a song. After the experimentation, the authors reported that the platform caused excitement and astonishment on the kids.

In addition to the use of robots for training intellectual abilities, they could also be used to develop spatial capabilities. For example, Tanaka and Takahashi (Tanaka \& Takahashi, 2012) designed a tangible interface for kids aged 3 to 6 in the form of a tricycle (see Figure 10-a) to remotely control a robot. When children moved with the tricycle, the same movements (i.e., forward, backward, left, right) were mapped to movements of the tele-operated robot. Despite the robot not being designed with this purpose in mind, this type of interfaces could be used to stimulate spatial mappings and develop spatial sense on kindergarten children.

One of the main advantages of using robots is that they can move. Therefore, they could be used to enhance physical development. Exploiting this idea, Tanaka et al. (Tanaka, Fortenberry, Aisaka, \& Movellan, 2005) presented a humanoid robot called QRIO. QRIO was designed to be introduced in a toddlers' classroom to make the kids move and dance, hence encouraging physical exercise. The interaction with this robot was however limited since it would dance autonomously to the music (see Figure 10-b) and react to the movements of a dancing partner (i.e, to his/her hand movements or clapping).

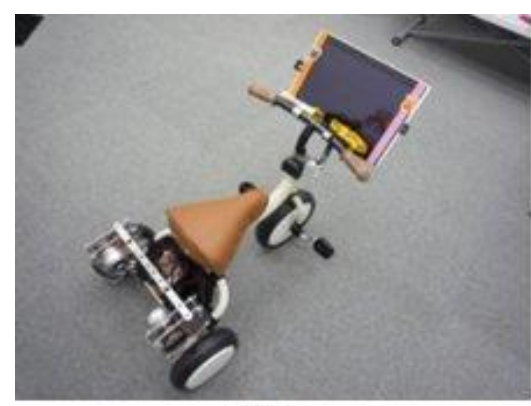

(a)

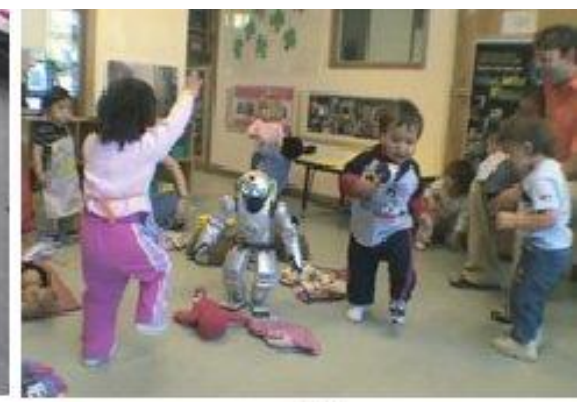

(b)

Figure 10. (a) Tricycle interface (extracted from (Tanaka \& Takahashi, 2012)). (b) Children dancing with QRIO (extracted from (Tanaka et al., 2005))

Another interesting approach of an enhanced toy to foster physical exercise and the training of spatial abilities is shown in the work by Garcia-Sanjuan et al. (GarciaSanjuan, Jaen, Nacher, \& Catala, 2015). In this case, the technological platform presented (see Figure 11) was aimed at supporting learning activities for prekindergarten children (up to 5 years) using a TUI-mediated mobile robot. Their results showed that the platform caused high excitement among children and most of the kids were able to drive the robot successfully. Moreover, children aged 3 or older are able to 
guide the robot through predefined paths (Nacher, Garcia-Sanjuan, \& Jaen, 2016a). Therefore, the interaction proposed was proven simple and intuitive enough for these specific and special users that are kindergarteners. Additionally, in their work the authors pointed out possible educational activities identified by actual educators in which the robot could be involved. For example, it could be used to train some psychomotor skills by making the robot move, possibly reproducing a sound to attract the attention of the children, and stimulate them to follow it. Additionally, the platform could be useful when teaching concepts such as "move/stop", "forwards/backwards", "left/right" or "quick/slow". Other learning activities could be designed by using physical objects that represent concepts to be learned and by asking children to drive the robot until reaching the position of said physically-represented concept.

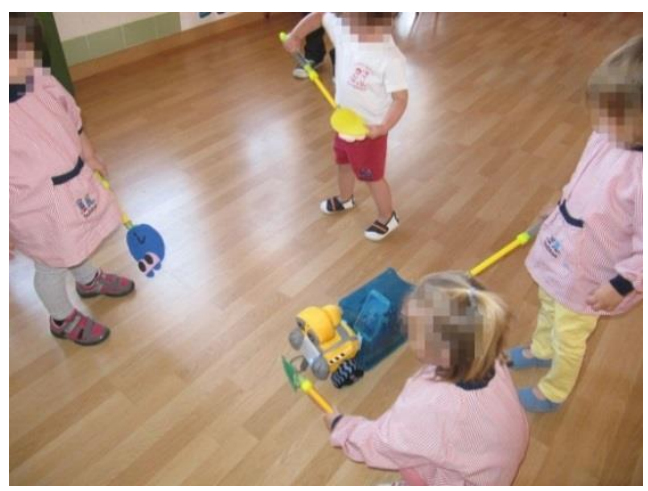

Figure 11. Children driving a mobile robot (extracted from (Garcia-Sanjuan, Jaen, Nacher, et al., 2015)).

The use of robots is also being considered with commercial purposes. Recently, Fisher Price presented Code-a-Pillar ${ }^{1}$, a robotic caterpillar to teach programing to children aged from 3 to 8 years. Several segments can be attached to the motorized head of the caterpillar and each one works as an instruction to be executed sequentially. Each block has a functionality such as go forward, go back, turn left/right or stop and play a song (see Figure 12). As Fisher Price points out, the Code-a-Pillar robot is aimed to inspire logical thinking and foster problem solving skills in the youngest.

\footnotetext{
${ }^{1}$ http://www.fisher-price.com/en_US/codeapillar/index.html
} 
Kindertivity: Usability and Communicability Strategies for Interactive Surfaces and PreKindergarten Children

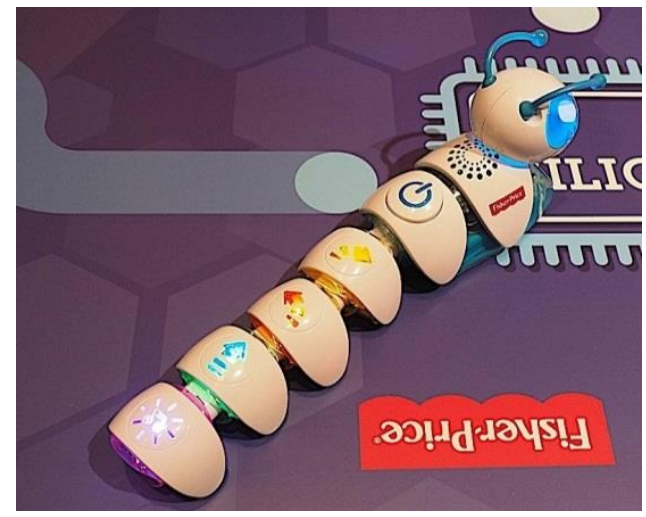

Figure 12. The Code-a-Pillar robot presented by Fisher-Price.

\subsection{Discussion}

In Table 1, the works listed above are classified in terms of several factors. On the one hand, the age of the users involved and the capacities, inferred from (Jefatura del Estado, 2006), that the proposed technologies can improve: physical development (P), socio-affective development (S) and cognitive and intellectual development (I). For each capacity there are several areas of improvement: related to physical development the analyzed works address physical exercise (P-p) and fine motor skills (P-f); in the social development we can identify the collaboration area (S-c); and in the cognitive and intellectual development we can find the areas that target spatial (I-s), linguistic (I1), logic and mathematic (I-m), and the exploration and discovery (I-e) skills. The works are also categorized by the technology used, namely computers (C), tablets (T), mobiles/smartphones (M), tabletops (TT) or robots (R). And, finally, the last dimension covers the type or means of interaction: tangible (T), keyboard (K), mouse (Mo), joystick (J), multi-touch (M), body gestural $(\mathrm{G})$ or vocal $(\mathrm{V})$.

\subsubsection{The Technological Evolution.}

The previous review of works suggests that not all the technologies considered fit in the same way into the children's development and the requirements that the educational activities should fulfill. The use of traditional computers limits the creation of educational activities targeted to preschool children since they are placed in a fixed location and this prevents designers from developing activities that involve children's movement along the space where the activity is been carried out. Moreover, traditional computers use a mediated interaction (mouse and keyboard) which is not very natural or intuitive for this specific type of users who have not fully developed their fine motor skills yet. In addition, the use of these mono-user mediated interactions with a mouse 
and a keyboard obstruct collaborative activities with children. The use of tabletop technologies solves several limitations of the traditional computers. Firstly, the way in which users interact with the technology changes to a direct-touch approach (tangible or tactile interaction), which, as Hourcade points out (Hourcade, 2007), is preferred over mediated pointing devices by children. Secondly, the tabletop's form factor and size offers more opportunities for supporting collaboration between peers than the traditional computers do. In this respect, several works have evaluated the suitability of tabletops for supporting collaboration (Fleck et al., 2009; Rick et al., 2009, 2010) and even for fostering creativity (Catala et al., 2012; Helmes et al., 2009). Regardless of these advantages, tabletops are also fixed to a location and are very expensive preventing the acquisition of large number of devices in education centers. Finally, the use of tablets and smartphones solves these two limitations. These devices allow the movement of children with the device along the area where the activity is being carried out. Besides, tablets have a much lower cost which makes them an affordable technology for most schools. Finally, looking at the year of publication of the works listed, there is a trend to leave the use of traditional computer and tabletop technologies behind and choose the tablets and smartphones for developing games for the youngest. Moreover, the works published in recent years also use robot technologies for developing educational games for preschoolers. Robots, as well as tablets and smartphones, can be moved (or move autonomously) around the space and foster children's movement and physical exercise. The interaction with robots is usually carried out by using tangible or gestural interaction overcoming the mediated interaction of traditional computers (Han, Jo, Park, \& Kim, 2005). Additionally, robots gather several people around simultaneously and support collaborative and cooperative activities. Seeing that the most emergent technologies when designing educational games for the youngest are robots, tablets and smartphones, the multi-touch and tangible interactions seem the most promising techniques that will need further research efforts to analyze their adequacy and limitations when applied to this specific type of users.

\subsubsection{A Future Challenge: Ubiquitous games.}

The reviewed technologies may be used simultaneously for creating Ambient Intelligence (AmI) environments that monitor children and adapt the activities to them depending on their actions in a natural and undetectable way (IST Advisory Group, 2001). A first step was made by Steurer and Srivastava (Steurer \& Srivastava, 2003) who presented a smart table that automatically monitors kid's interaction (with tangible blocks) on the surface and enables educators to follow the children's learning progress. Another example in this direction is the work by Bobick et al. (Bobick et al., 1999) with the KidsRoom which immerses children in a fantasy adventure in which children must cooperate together to explore a fantasy story. The story goes ahead when cooperative actions are made by children (e.g. rowing a virtual boat or yelling a magic 
Kindertivity: Usability and Communicability Strategies for Interactive Surfaces and PreKindergarten Children

word) and the actions are captured with cameras and microphones in the room (Figure $13)$.

Table 1. Comparison of works

\begin{tabular}{|c|c|c|c|c|c|}
\hline Work & $\begin{array}{l}\text { Age } \\
\text { (years) }\end{array}$ & Capacities & Areas & Technology & Interaction \\
\hline (Tanaka et al., 2005) & $0-2$ & $\mathrm{P}$ & P-p & $\mathrm{R}$ & $\mathrm{T}, \mathrm{G}$ \\
\hline (Jones \& Liu, 1997) & $2-3$ & $\mathrm{I}$ & $\mathrm{I}-1, \mathrm{I}-\mathrm{m}$ & $\mathrm{C}$ & $\mathrm{K}$ \\
\hline (Nacher et al., 2015) & $2-3$ & $\mathrm{P}$ & P-f & $\mathrm{T}$ & M \\
\hline (Nacher et al., 2014) & $2-3$ & $\mathrm{P}$ & P-f & $\mathrm{T}$ & M \\
\hline (Nacher \& Jaen, 2015a) & $2-3$ & $\mathrm{P}$ & P-f & $\mathrm{T}$ & M \\
\hline $\begin{array}{l}\text { (Nacher, Jaen, \& Catala, } \\
\text { 2014) }\end{array}$ & $2-3$ & I & $\mathrm{I}-1$ & $\mathrm{~T}$ & M \\
\hline (Nacher et al., 2017) & $2-3$ & I & $\mathrm{I}-1$ & $\mathrm{~T}$ & M \\
\hline (Hiniker et al., 2015) & $2-5$ & I & I-1 & $\mathrm{T}$ & M \\
\hline $\begin{array}{l}\text { (Garcia-Sanjuan et al., } \\
\text { 2015) }\end{array}$ & $2-5$ & $\mathrm{I}, \mathrm{S}$ & I-s, S-c & $\mathrm{R}$ & $\mathrm{T}$ \\
\hline $\begin{array}{l}\text { (Nacher, Garcia-Sanjuan, et } \\
\text { al., 2016a) }\end{array}$ & $2-5$ & $\mathrm{I}, \mathrm{S}$ & I-s, S-c & $\mathrm{R}$ & $\mathrm{T}$ \\
\hline (Strommen et al., 1996) & 3 & $\mathrm{P}$ & $P-f$ & $\mathrm{C}$ & Mo, J, B \\
\hline (Marco et al., 2009) & $3-4$ & $S$ & S-c & TT & $\mathrm{T}$ \\
\hline $\begin{array}{l}\text { (Marco, Cerezo, } \quad \& \\
\text { Baldassarri, 2009) }\end{array}$ & $3-4$ & $\mathrm{~S}$ & S-c & TT & $\mathrm{T}$ \\
\hline (Mansor et al., 2009) & $3-4$ & I & I-e, S-c & TT & M \\
\hline $\begin{array}{l}\text { (Khandelwal \& Mazalek, } \\
\text { 2007) }\end{array}$ & $3-5$ & I & $\mathrm{I}-\mathrm{m}$ & TT & $\mathrm{T}$ \\
\hline (Vatavu et al., 2015) & $3-6$ & $\mathrm{P}$ & $P-f$ & $\mathrm{~T}-\mathrm{M}$ & M \\
\hline (Ghosh \& Tanaka, 2011) & $3-6$ & I & $\mathrm{I}-1$ & $\mathrm{R}$ & $\mathrm{V}, \mathrm{G}$ \\
\hline (Tanaka \& Matsuzoe, 2012) & $3-6$ & I & I-1 & $\mathrm{R}$ & $\mathrm{V}, \mathrm{G}$ \\
\hline $\begin{array}{l}\text { (Tanaka \& Takahashi, } \\
\text { 2012) }\end{array}$ & $3-6$ & I & I-s & $\mathrm{R}$ & $\mathrm{T}$ \\
\hline (Chiong \& Shuler, 2010) & $3-7$ & I & I-1 & $\mathrm{T}$ & M \\
\hline $\begin{array}{l}\text { (Navarro-Newball et al., } \\
\text { 2014) }\end{array}$ & $3-11$ & I & $\mathrm{I}-1$ & $\mathrm{C}$ & $\mathrm{V}$ \\
\hline (Ahlström \& Hitz, 2013) & $4-5$ & $\mathrm{P}$ & P-f & $\mathrm{C}$ & Mo \\
\hline (Berggren \& Hedler, 2014) & $4-5$ & $\mathrm{I}, \mathrm{S}$ & $\mathrm{I}-\mathrm{m}, \mathrm{S}-\mathrm{c}$ & $\mathrm{T}$ & M \\
\hline (Zaranis et al., 2013) & $4-6$ & I & $\mathrm{I}-\mathrm{m}$ & $\mathrm{T}$ & M \\
\hline (Kammer et al., 2014) & $4-6$ & $\mathrm{I}, \mathrm{P}$ & $\mathrm{I}-, \mathrm{P}-\mathrm{p}$ & TT & M \\
\hline (Tyng et al., 2011a) & $4-6$ & I & $\mathrm{I}-\mathrm{m}$ & TT & M \\
\hline (Huber et al., 2016) & $4-6$ & I & $\mathrm{I}-\mathrm{s}$ & $\mathrm{M}$ & M \\
\hline (Soute \& Nijmeijer, 2014) & $4-6$ & I & I-1 & $\mathrm{R}$ & G \\
\hline (Shen et al., 2013) & $4-6$ & I & $\mathrm{I}-1$ & $\mathrm{R}$ & $\mathrm{T}$ \\
\hline (Tyng et al., 2011b) & $5-6$ & I & $\begin{array}{l}\mathrm{I}-1, \mathrm{I}-\mathrm{s} \\
\mathrm{I}-\mathrm{m}\end{array}$ & $\mathrm{TT}$ & M \\
\hline (Sluis et al., 2004) & $5-7$ & $\mathrm{I}, \mathrm{S}$ & $\mathrm{I}-1, \mathrm{~S}-\mathrm{c}$ & TT & M \\
\hline (van Daal \& Reitsma, 2000) & $6-7$ & I & I-1 & $\mathrm{C}$ & Mo, K \\
\hline
\end{tabular}


Another example is the work by Chen et al (Chen et al., 2002) who presented SmartKG, a sensor-instrumented environment for early childhood education. The system uses unobtrusive sensors (microphones, video cameras and motion detectors) to continuously monitor the interactions between children, teachers and the objects located in the classroom. In addition, a sensor-instrumented badge has to be worn by children and teachers in order to provide to the system meaningful location information. The environment collects, interprets and presents the data in a userfriendly way for helping educators to widely explore the children learning process through both, their progress in the proposed activities and their social behavior. In the same line of providing children's information to teachers, Hwang et al (Hwang et al., 2012) proposed a system to monitor children's behavior in out-of-classroom activities like field trips. In this case, children worn a backpack equipped with a sensor mote and a smartphone for sensing the data (see Figure 14). Moreover, the authors tried to include a camera in a cap in order to have accurate data about children's point of view but it was too obtrusive for children since a cap is not a regular clothing accessory for them.
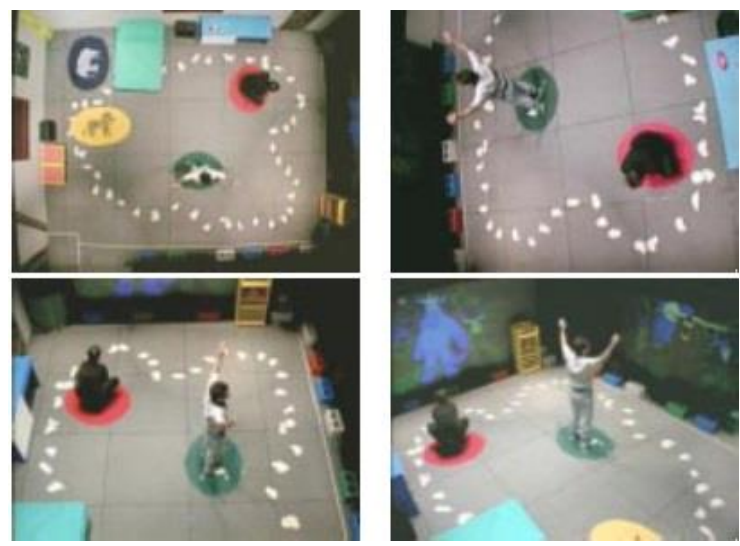

Figure 13. The Kidroom from the three cameras used to sensor (extracted from (Bobick et al., 1999)).

These systems are a step forward in the development of ambient intelligent environments but they are unable to adapt dynamically the characteristics of the learning/game environment by analyzing the context information obtained by the installed sensor infrastructure. 
Kindertivity: Usability and Communicability Strategies for Interactive Surfaces and PreKindergarten Children

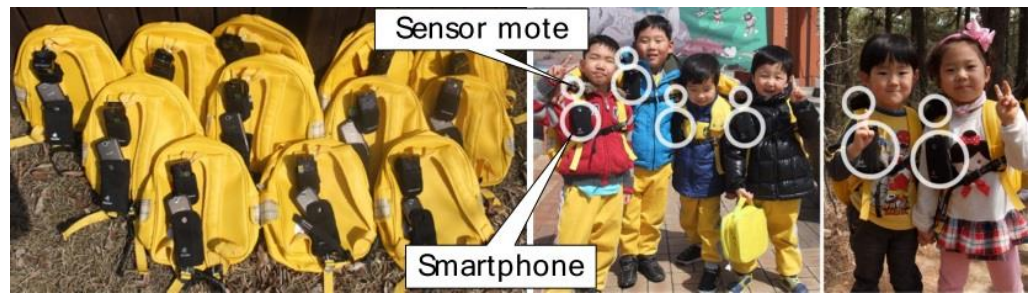

Figure 14. The backpack used for tracking in (Hwang et al., 2012).

Going over the previous list of works that address the design of a ubiquitous game, we can see that some of them are outdated and most of them are focused in monitoring children and provide their data to teachers and not to develop an environment that enriches children's activities through the use of technologies. Hence, new approaches are needed that take advantage of the newest technologies available to design ambient intelligent games that support children's development and satisfy children's expectations when playing. These new approaches should use the new technologies to augment the real space but not replace the natural and real-world activities which children are comfortable with. Moreover, these environments should provide fully engagement activities and immersion at the same time that support collaborative play and fosters creativity and imagination in children. According to the works reviewed above, these characteristics could be achieved with the technologies that have been discussed here and, in this respect; the current state of $\mathrm{HCI}$ is quite advanced to provide natural interactions. However, as Cook et al (Cook, Augusto, \& Jakkula, 2009) pointed out, AmI environments should also sense users' actions and the state of the activity, reason about this information and, finally, make decisions and act accordingly to this information. In this respect, there remains a long way of research in the areas of sensing children movement and developing Artificial Intelligence algorithms that use this information for acting accordingly. These areas should be the focus of future research to enable the creation of AmI games targeted to the youngest.

\subsubsection{The Interplay between Age, Cognitive Skills and Interaction Modalities}

On the other hand, regarding the dimensions of development that the reviewed works address, there are a great number of efforts put into the development of the physical and intellectual capacities of children. As for the physical capacities, most works present activities and games that could give support to the development of fine motor skills. However, in this dimension few works have been proposed with preschool children when developing games that support their gross motor skills or promote health and wellbeing through performing physical activity and active play. In our opinion, it will be interesting to develop games that support this type of development and we think that tablets, smartphones and robots are the best technological platforms for supporting the gross motor skills development since they have the ability to be moved from one 
place to another and, hence, allow children's movement. Regarding the cognitive and intellectual dimension, most works focus on developing games that foster the logic, mathematic and linguistic skills. Hence, there is a lack of works that develop games for giving support to the development of spatial abilities or games supporting creativity, exploration and discovery. According to this, future work should be done to develop and design games that support these dimensions of children' development.

On the other hand, despite there are several studies recognizing the social value of play and game participation (Mead, 1934) (Piaget, 1975) and there are several works that point out the suitability of the new technologies, such as tabletops, tablets, smartphones and robots for collaborative playing, and children playing together can beat playing alone (Inkpen, Booth, Klawe, \& Upitis, 1995), the development of games that support social and affective skills in preschool children is not fully exploited. Hence, a future work to be addressed in the area is the use of these technologies for designing games that allow, support and foster cooperative and collaborative play. Finally, none of the works explored the areas in the social-affective dimension. Hence, future games targeting preschooler's development could focus on giving support to the development of self-awareness, self-regulation and emotional intelligence of children as proposed in (García, Vela, Rodríguez, Arcos, \& Zea, 2015) (González-González \& NavarroAdelantado, 2015) and provide the caretakers and educators with a method to evaluate these emotions in children's playing (Padilla-Zea, Sánchez, Vela, Abad-Arranz, \& López-Arcos, 2012). These unexplored areas will have to be the focus of intense research in the near future to create games that support all the dimensions of preschool children's development identified.

Regarding the cognitive skills supported in each age, according to the data of Table 1, when the targets are three years-old or younger children there is a trend to design game technologies supporting physical and intellectual dimensions of children development. However, for children older than 3 years the reviewed games mostly support social and intellectual activities. Although the spatial and linguistic areas of the intellectual dimension are commonly supported for all the age range evaluated, the logic and mathematic area is only supported for children older than 3 years.

Finally, analyzing the type of interaction used when designing game technologies for kindergarten children, we can observe that for children aged 4 years and younger the most common ways of interaction are both the tangible and the touch interaction. However, it is not the case for older children since despite the predominance of the touch interaction in games targeted to them; new means of interaction such as mouse, keyboard, vocal and body gestural emerge with these users taking advance of their more developed cognitive and motor skills. 
Kindertivity: Usability and Communicability Strategies for Interactive Surfaces and PreKindergarten Children

\subsection{Conclusions}

Summing up, the contributions of this paper are manifold. First, a review of the current state of the art of technology-aided activities that support pre-kindergarten and kindergarten children's development in three dimensions (physical, socio-affective and cognitive) has been made. The analysis of the existent literature reveals that game technologies are suitable for supporting the improvement and development of very young children capacities. The second contribution is a set of future challenges that list the unexplored areas of preschool children's development in each technology in which game technologies may have a real and noticeable impact. These identified areas have to be the focus of intense research in the future to create games that support all the dimensions of preschool children's development effectively. Moreover, the most emergent technologies for developing educational games for prekindergarten and kindergarten children were identified. Further, this work points out the need of future research combining technologies for giving support to Ambient Intelligence educational games that promote children's development.

Finally, since pre-kindergarten and kindergarten children are in their pre-operational phase of their development, in this paper we have focused in the review of interaction mechanisms that have been used in the past to support different forms of gameplay in preschool instruction. The analysis of the effectiveness of these interaction mechanisms is crucial for the creation of future applications usable by them. However, educators and game designers would also take into account other aspects such as gameplay, effectiveness of the learning, user adaptation, narrative or emotional experience when choosing or developing game experiences for this specific type of users.

\section{Acknowledgments}

This work received financial support from Spanish Ministry of Economy and Competitiveness and funded by the European Development Regional Fund (EDRFFEDER) with the project TIN2014-60077-R (SUPEREMOS). This work is also supported by a pre-doctoral fellowship within the FPU program from the Spanish Ministry of Education, Culture and Sports to V. Nacher (FPU14/00136) and from GVA (ACIF/2014/214) to F. Garcia-Sanjuan. 


\title{
Chapter 3
}

\section{Multi-touch Technology in Early Childhood: Current Trends and Future Challenges}

Vicente Nacher, Javier Jaen

ISSI Group, Dpto. Sistemas Informáticos y Computación, Universitat Politècnica de València.

Published in Proceedings of the XVI International Conference on Human Computer Interaction (Interacción '15), pp.49-53. ACM (2015)

\begin{abstract}
The advantages of the direct manipulation style make the multi-touch technology an ideal mechanism to support learning activities for children. Moreover, although prekindergarten children are becoming frequent users of the technology little work has been done in the area to assess their actual abilities. This paper goes over the state of the art of multi-touch technology targeting pre-kindergarten children and its use for educational purposes. In addition, in this work we present future challenges that should
\end{abstract}


Kindertivity: Usability and Communicability Strategies for Interactive Surfaces and PreKindergarten Children

be faced in the area in the near future to establish the basis on which designers will develop educational applications for children that fully exploit the multi-touch technology according to the actual abilities of pre-kindergarten children.

\subsection{Introduction}

Multi-touch technology has rapidly evolved in recent decades and nowadays it has widespread acceptance (Buxton, 2013) because it provides users with a more natural and intuitive way to interact (Smith et al., 2012). As pointed out in (Common Sense Media, 2013) children between zero and eight years old are frequent users of digital media in the USA and they meet with touch technology often before they can even speak. Supporting these ideas the Horizon report (Johnson et al., 2012) places mobile devices, such as tablets and smartphones, as one of the two emerging technologies suitable for children aged under 2 years.

The three basic ideas behind the direct manipulation concept were listed by Shneiderman and Plaisant (Shneiderman et al., 2009): (1) the visibility of objects and actions of interest; (2) the replacement of typed commands by pointing-actions on objects of interest; and (3) the rapid, reversible and incremental actions that help children to keep engaged, giving them control over the technology and avoiding complex instructions.

A detailed analysis of applications in the Apple store made by Shuler (Shuler, 2012) reveals the growing use of educational applications for children based on touch devices. This is specially the case for preschool children (Figure 15) who were the target users of nearly $60 \%$ of these applications by 2011 .

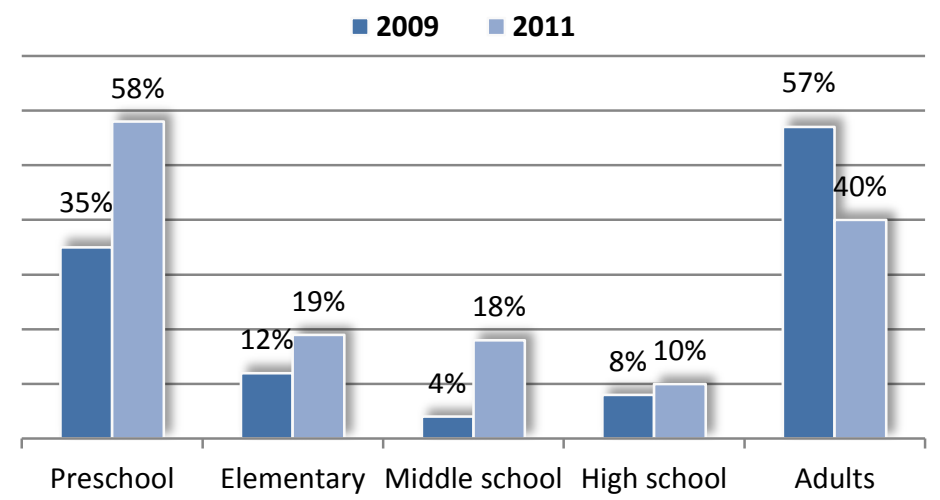

Figure 15. Target age 2009 vs 2011 for Education cat. in the Apple Store (Shuler, 2012).

However, the increasing interest in multi-touch technology has not given rise to studies on the design of multi-touch systems for the youngest age range (Hourcade, 2007). Moreover, the lack of standardized and universally accepted interactions for these 
challenging users makes the design of well-designed multi-touch interactions even more crucial (Ingram, Wang, \& Ribarsky, 2012).

According to these facts, in this paper we carry out a review of the current state of the literature of multi-touch technology with pre-kindergarten children and provide a set of future challenges to be addressed in the future. The end goal is to define a research agenda to make this technology usable by pre-kindergarten children and give application designers the necessary guidelines to develop touch applications according to the actual skills of pre-kindergarten children.

\subsection{State of the art}

Until very recently, there have been no research efforts addressing multi-touch interaction with pre-kindergarten children (aged less than three years). Possibly this has been the case because age is a limiting factor for experimental studies; young children do not have the verbal and cognitive skills to express their likes and preferences (Kremer, 2012) and, they are not able to carry out tasks for long periods or are easily distracted (Egloff, 2004). However, according to Piaget (Piaget, 1973), children are in a preoperational stage from 2 years old onwards, i.e., they begin to think in terms of images and symbols, and develop symbolic play with imaginary objects, which means they could be candidates for multi-touch technology. In addition, being aware of children developmental abilities is critical when designing software for the very young (Wolock, Ann Orr, \& Buckleitner, 2006).

This has motivated recent studies that assess the abilities of pre-kindergarten children to perform basic touch gestures. The work by Nacher et al (Nacher et al., 2015) reveals that the advantages and features of multi-touch technology is not being fully exploited in existing commercial applications since only the drag and tap gestures are being used and no support for collaboration is given. Hence, the work evaluates a set of basic touch gestures with children aged from two to three years (Figure 16) concluding that even these young users are able to perform a set of basic touch gestures such as tap, drag, scale (up \& down) and one finger rotation. In addition, in this work a set of design guidelines is proposed to deal with complex gestures such as double tap and long pressed. These assisted strategies were evaluated later in (Nacher, Jaen, Catala, et al., 2014) and results show that even these more complex gestures can be successfully performed by pre-kindergarten children with basic assistance.

In the experimental studies in (Abdul Aziz, Batmaz, Stone, \& Paul, 2013) and (Abdul Aziz, Mat, Batmaz, Stone, \& Paul, 2014) four applications are considered and the interaction needed to play with them is evaluated. The gestures under test are the tap, drag, rotate, drag and drop, pinch, spread and flick and the experiment involves children aged 2 to 4 years. According to the results, the authors conclude that children aged four are able to perform all the evaluated gestures. Those aged three only find problematic the spread task. Finally, those aged two are able to perform the tap and 
Kindertivity: Usability and Communicability Strategies for Interactive Surfaces and PreKindergarten Children

drag gestures properly, learn quickly the flick gesture but they are less effective with the more complex gestures such as drag and drop and pinch.

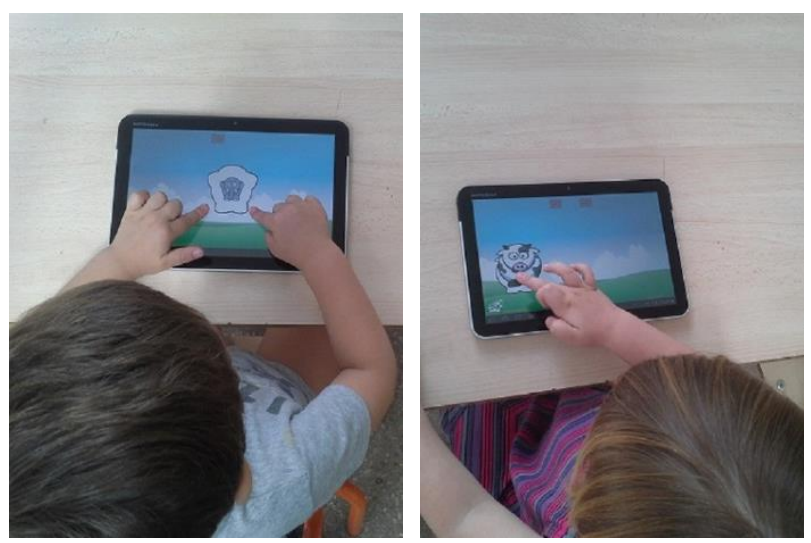

Figure 16. Examples of scale up and drag tests (extracted from(Nacher et al., 2015)).

Another interesting study is the work of Vatavu et al (Vatavu et al., 2015) who evaluate touch gestures with children between 3 and 6 years using tablets and mobile devices. They conclude that despite there is a significant performance growth with age, in general, children have good performance with the tap, double tap and single hand drag and drop gestures. However, in the case of the double hand drag and drop gesture (see Figure 17) they do not reach these good results and the success rate drops to $53.7 \%$. In addition, authors correlate the results with a sensorimotor evaluation based on children's finger dexterity and their graphomotor and visuospatial processing abilities. The correlation shows that children with higher visuospatial skills (i.e. having better skills for understanding relationships between objects, as location and directionality) perform better in the drag and drop tasks. Furthermore, the study also reveals that children with more developed visuospatial skills tap closer to the center of targets in the tap and double tap tasks since they have a better understanding of the targets' geometries.
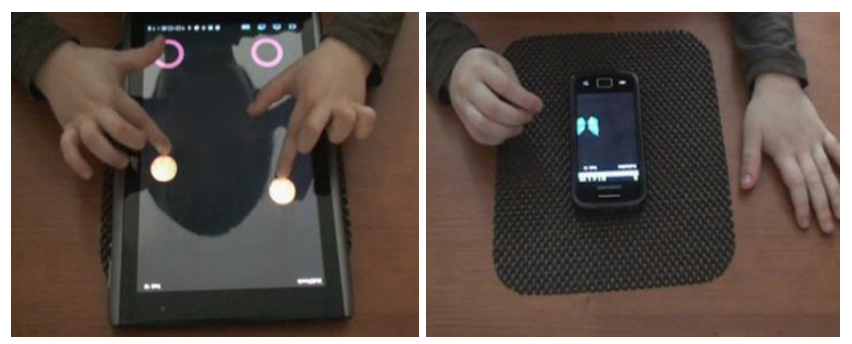

Figure 17. Child performing double drag gestures (extracted from (Vatavu et al., 2015)). 
Usability is not the only dimension that has been addressed by recent studies in the field. Another topic of interest is that of communicability when pre-kindergarten children are considered. The work in (Nacher, Jaen, \& Catala, 2014) report a first approach to evaluate mechanisms for applications to communicate pre-kindergarten children the expected multi-touch gestures at a given moment. In this study, the authors present and evaluate two visual approaches (iconic and animated) to communicate touch gestures (see Figure 18). Three touch gestures are considered: the tap representing in-place gestures (i.e. those in which the hand does not actually describe a trajectory but taps at a very specific pace or in a specific way); the drag representing one-contact point gestures that require a movement following a specific trajectory; and the scale up gesture representing two-contact point gestures that require movement. The results show, firstly, that none of the evaluated languages is effective to communicate in-place gestures and, secondly, that the animated approach overcomes the iconic one for gestures that require movement of contacts reaching success rates above $85 \%$. This fact suggests that even pre-kindergarten children are able to interpret the direct mapping between the visual stimuli (i.e. a hand sliding on the surface) and the gesture to be performed. Therefore, this work suggests that visual languages could be an effective way to enable pre-kindergarten children autonomous interaction.

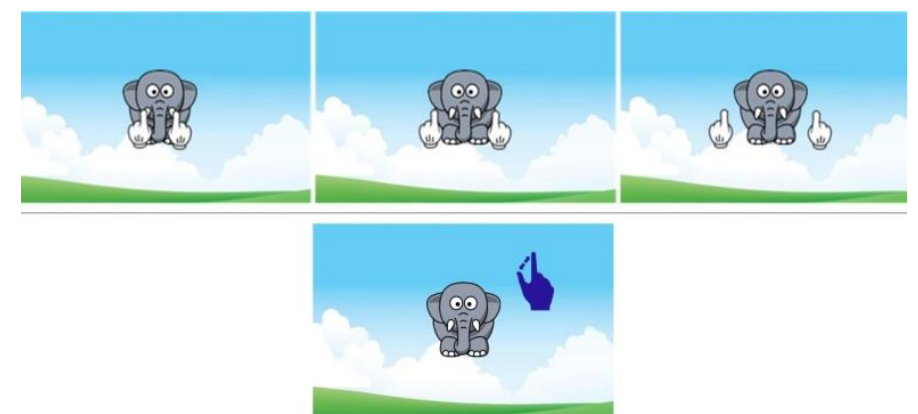

Figure 18. Description of the animated visual (top) and the iconic (bottom) language for the scale up gesture (extracted from (Nacher, Jaen, \& Catala, 2014)).

Exploring the educational dimension and the suitability of multi-touch surfaces to support educational activities there are several works that strengthen the idea that this technology provides benefits for pre-kindergarten children education. For example, the work by Bebell et al (Bebell, Dorris, \& Muir, 2012) shows a nine week study comparing the improvement of kindergarten children taking early literacy lessons when using tablets for learning or using a traditional non-technological method. Their results show that the group that learned with tablets scored higher on early literacy assessments, particularly these higher scores are present in the ability to recognize sounds and represent sounds and letters. Following the same line, Chiong and Shuler (Chiong \& Shuler, 2010) conduct an experiment involving touch devices and audiovisual material adapted to children aged three to seven years and their results show that children obtain remarkable gains in vocabulary and phonological awareness. 
Kindertivity: Usability and Communicability Strategies for Interactive Surfaces and PreKindergarten Children

Moreover, Knoche et al (Knoche, Rasmussen, \& Boldreel, 2014) point out that the interaction of children aged between 16 and 33 months with interactive elements in a tablet does not reduce their comments in dialogic reading activities. Another example is provided by Zaranis et al (Zaranis et al., 2013) who conduct an experiment to study the effectiveness of digital activities on smart mobile devices (tablets) to teach mathematical concepts such as general knowledge of numbers, efficient counting, sorting and matching with kindergarten children. Their results show that the tabletaided learning provided better learning outcomes for the students than the traditional teaching method. Kammer et al (Kammer et al., 2014) present three applications to foster the development of cognitive and motor skills on a multi-touch tabletop with children aged from four to six years. The conducted experiment shows that even preschool children are able to use this technology and they enjoy the task and collaborate in the multi-user activity.

The results of these works suggest that pre-kindergarten children are prepared to use multi-touch technology and the intuitive and natural interaction of direct manipulation style of the multi-touch technology makes it ideal to support pre-kindergarten children interaction and, hence, educational activities targeted to them. Moreover, these works conclude that these particular users are able to perform a set of touch gestures successfully and future applications designed for them do not need to be restricted to only basic interactions such as the drag and tap gestures. However, these works reveal that there is no consensus or standardization of the multi-touch interaction style for users in these early ages.

\subsection{Future challenges}

In this section we present a compilation of future works that we consider interesting and necessary to complete the literature and shed light on the specific needs of prekindergarten children when using multi-touch technology.

On the one hand, according to Hinrichs and Carpendale (Hinrichs \& Carpendale, 2011) who point out that there is evidence that some events are affected by previous and subsequent ones and given that all the analyzed studies consider and evaluate the gestures in isolation, an interesting strand of future work would be to evaluate these same gestures that pre-kindergarten can do in isolation but when several interactive elements are shown simultaneously. Users should perform different preset sequences of these gestures in order to determine whether the cluttering of elements in the interaction or the task chaining affect their performance. These results will help to develop more complex applications which are not restricted to only one possible interaction in each phase of the game.

Another interesting issue that remains to be addressed is the processing of the unexpected touch events when children are holding the tablet with a finger resting on the display or when part of the palm also touches the surface. This issue is difficult to 
address because children may not be aware of such unintentional contacts with other parts of their body when their fingers approach the screen resulting in an unexpected effect. It would therefore be interesting to explore potential improvements in multitouch usability, for instance by determining and filtering out unexpected blob contacts wherever applicable.

Addressing the topic of the definition of effective mechanisms to communicate which actions are expected from the user; and taking as a starting point the work (Nacher, Jaen, \& Catala, 2014), there is still a great deal of work to do to assess the best approaches. This preliminary study points out that animated languages can be effective to communicate touch gestures to pre-kindergarten children and help them to be autonomous when using the multi-touch devices. However, as pointed out by the authors, only three gestures were evaluated, hence, it remains to be evaluated whether the inclusion of additional gestures has an impact on the overall performance and effectiveness of the languages. Moreover, the gestures were tested in isolation; therefore, languages should be studied when the interaction area is cluttered with many touchable elements and their corresponding visual cues or with elements that may be manipulated with several different gestures. With respect to communicability of touch gestures, another possible future work can be the evaluation of other languages both iconic and animated in order to find out which type of language fits better to communicate touch gestures to infants. In addition, an interesting future work could be to design and evaluate a similar approach to the method used by Balonian et al (Baloian, Pino, \& Vargas, 2013) for children between five and six years old. In this work, each gesture was associated to a specific character in a way that the gestures were "recallable". Metaphors such as a walking ladybug for a drag gesture or a jumping grasshopper for a double tap were used. Finding suitable metaphors that prekindergarten children can understand could be very useful to develop autonomous educational applications.

The studies in the literature point out that pre-kindergarten children have the necessary skills to make use of multi-touch technology. However, these works implement assistive techniques to deal with precision issues during the initiation and termination phases of gestures since they assume that pre-kindergarten children are no able to perform the gestures with high accuracy. This causes that the implemented interaction styles do not allow children to have the control over the termination of the gestures despite they are in the process of developing their motor skills and some of them may have already the proper cognitive abilities to perform the gestures with higher levels of precision. As a result, existing applications designed under these assumptions do not benefit from the use of multi-touch technology to help children to develop their precision-related cognitive and motor skills. According to this observation it would be interesting to evaluate dynamic gestures (those that require movement of contacts over the surface) demanding high levels of accuracy to users, specifically, it would be interesting to evaluate how accurately they can rotate an object, how close they can drag an object to a target and whether they are able to perform scaling gestures (up and 
Kindertivity: Usability and Communicability Strategies for Interactive Surfaces and PreKindergarten Children

down) with enough accuracy to stop the stroke of the gesture in a specific moment to reach a desired size. This would certainly help in understanding the limitations on accuracy that should be fulfilled in applications targeting pre-kindergarten children. In addition, the data collected during the experimentation could be used to develop assistive strategies to deal with precision issues in an adaptive way for the users that actually need it and not in an exhaustive way for every child as current systems do.

Finally, once known the actual capabilities and abilities of pre-kindergarten children, the gestures that they can perform, the accuracy that they can achieve and evaluated the communicative strategies suitable for them; interesting future works could be developed for the definition, construction and evaluation of environments based on multi-touch technology that foster creativity and allow collaboration between peers. These environments could be integrated into classrooms and be used with educational purposes to allow children to develop their creativity skills and allow educators to monitor the progress of their students and create appropriate content for them. As Rushton (Rushton \& Juola-Rushton, 2008) points out, the creation of classroom environments that enhance students expression and selection opportunities provides a proper atmosphere towards helping children to learn at their own pace. A starting point could be taking advance of (1) the portability of tablets that allow children and teachers to use various locations in the classroom enabling the creativity and collaboration of small groups of children; and (2) the more sophisticated interface of tablets that allows both individual and mutual interaction between two or more users (Wakefield \& Smith, 2012).

\subsection{Conclusions}

The contributions of this paper are twofold; firstly, a review of the state of the art of multi-touch technology for pre-kindergarten children was made. The literature suggests that the use of this technology is an ideal way to develop applications for these very young users. In addition, the presented studies show that pre-kindergarten children are able to perform more touch gestures than could be expected when analyzing existing commercial applications. On the other hand, pre-kindergarten children are developing their motor skills and, hence, in order to use the multi-touch technology, designers should be aware of the actual capabilities of children when developing interfaces for them. The second contribution is a collection of future challenges to be faced in the near future when building multi-touch technology for preschool children. These challenges are related to communicability, adaptability and usability of multi-touch applications designed for these challenging users. We hope that the usability studies and design guidelines compiled from these works and the future challenges discussed in this paper will allow designers of future applications for pre-kindergarten children to fully exploit the potential of multi-touch technology to support their cognitive and motor development. 
Multi-touch Technology in Early Childhood: Current Trends and Future Challenges

\section{Acknowledgments}

Work supported by the MINECO (grants TIN2010-20488 and TIN2014-60077-R) and from GVA (ACIF/2015/075). 



\section{Chapter 4}

\section{KINDERTIVITY: \\ Using Interactive}

Surfaces to Foster

Creativity in Pre-

\section{kindergarten Children.}

Vicente Nacher, Javier Jaen

ISSI Group, Dpto. Sistemas Informáticos y Computación, Universitat Politècnica de València.

Published in Proceedings of the XVI International Conference on Human Computer Interaction (Interacción '15), pp.135-136. ACM (2015)

\section{Abstract}

Taking into account the existent educative and pedagogical techniques, which have proved its effectiveness to foster the innovation and creativity, this thesis poses to 
Kindertivity: Usability and Communicability Strategies for Interactive Surfaces and PreKindergarten Children

develop, experiment and evaluate a new technological framework based on interactive surfaces to be applied in the context of preschool education. The goal is to facilitate the three factors required for creative learning: knowledge, creative thinking and motivation but taking into account the cognitive and interaction limitations of these very young users.

\subsection{Introduction}

The European Union recognizes creativity as a key factor for the economic development, hence, "Increase the creativity and the innovation, including the entrepreneurship spirit, in all levels of the education and training" is the $4^{\text {th }}$ goal of the strategic framework for the European cooperation in the field of education and training and it is one of the constituent elements of the new R\&D strategy in the 2020 Horizon (Comisión Europea, 2009). This is a necessary strategy because, as Cropley points out (Cropley, 2001), the traditional education systems tend to frequently assume the existence of one valid answer (or, at least, a preferred one) for any type of problem. This fact prevents the generation of new ideas and innovation processes. According to this, a creative student, or with a different cultural basis, can be considered as a distortion source or distraction in the knowledge acquisition process imparted by the teacher. However, while the individual ability to obey exactly the given orders with discipline were in line with necessities of an industrial society and massive production systems, the ability to be part of collaborative processes and direct the divergent thinking as the motor of creativity and innovation are essentials nowadays in the information society.

Fostering creativity must be addressed from a very early age, even in the preschool phase, since the main cognitive processes associated to creativity have their sources in this phase of the individual development (Damon, Lerner, Kuhn, \& Siegler, 2006). Moreover, the technological support to be used when developing creative characteristics in pre-kindergarten children is critical since the choice of the underlying supporting technology has a great impact on the nature of the pedagogical activities to be performed in a creative learning context.

The initial hypothesis for this thesis is that it is possible to trigger in a more effective way creative collective constructivist processes in pre-kindergarten children through the participation of multiple users in physical spaces of shared games based on interactive surfaces. Therefore, we pretend to study the use of these surfaces in the early phase of the cognitive development and its suitability for the creation of educational tools. 


\section{2. $\quad$ Related work}

Multi-touch technology has evolved quickly in recent years, from the adoption of graphical user interfaces to its wide acceptance nowadays (Buxton, 2013). This technology offers new sophisticated input and processing mechanisms that enable users to interact in a more natural and intuitive way (Smith et al., 2012). These characteristics have triggered a new approach to developing applications for even very young children. Supporting this evidence, the Horizon report (Johnson et al., 2012) identifies mobile devices (smartphones and tablets) as one of the two emerging technologies suitable for children under two years old and Rideout pointed out that children between zero and eight years old are frequent users of digital media and they meet with touch technology often before they can even speak (Common Sense Media, 2013).

This inherent ability of touch systems to engage children's attention is being widely exploited to promote learning activities from pre-kindergarteners to adolescents (Mansor, De Angeli, \& De Bruijn, 2008), (Sluis et al., 2004), (Khandelwal \& Mazalek, 2007). Moreover, some studies have demonstrated that the technology can also be used to promote collaboration between peers (Rick et al., 2010)(Rick \& Rogers, 2008), (Fleck et al., 2009) and to foster creativity (Helmes et al., 2009), (Catala et al., 2012).

However, the increasing interest in multi-touch technology has not as yet given rise to studies on the design of multi-touch systems for the youngest age range (2-3 years old) (Hourcade, 2007).

Taking into account the previous works, we believe our proposal is a step forward because of the following reasons: (1) the suitability of the interactive surfaces to support social learning since several subjects share the same physical space and, as it happens in traditional technology-free games, the communication during the creation process, experimentation and reflection is direct and no mediated by a computer. (2) The collaborative nature of the technologic infrastructure, in which users can carry out different tasks in parallel and on the same table; and (3) the creative nature of the infrastructure in which users select the game elements and the reactive behavior that its offer. This allows educators to have a direct feedback about the evolution of children's creative mental models. These models are internalized in a collective way since the activity itself is based on reflection, creation and experimentation processes. The goal is that also educators have a tool on which they can measure in an effective way the level of knowledge development, in depth and breadth, of the divergent/convergent thinking processes and the motivation of users in the activity.

\subsection{Contribution}

In order to assess the actual skills of pre-kindergarten children with interactive surfaces, we have performed an evaluation of a set of basic multi-touch gestures 
Kindertivity: Usability and Communicability Strategies for Interactive Surfaces and PreKindergarten Children

(Nacher et al., 2015). The results showed that although only the tap and drag gestures are used in commercial applications targeted to pre-kindergarten children, there are additional multi-touch gestures that can be performed by them (one finger rotation, scale up and scale down). In addition, this study provides a set of design guidelines to define and improve the interactions of these particular users. The application of several of these design guidelines (Nacher, Jaen, Catala, et al., 2014) showed that more problematic gestures, such as double tap and long press, can be suitable for prekindergarten children too.

On the other hand, we have carried out another study addressing communicability of multi-touch gestures (Nacher, Jaen, \& Catala, 2014) and the results proved that the use of animated languages to communicate gestures to pre-kindergarten children is possible. This opens a new opportunity to new studies with training sessions in order to evaluate the acquisition ability of this type of languages with these young users.

These preliminary results have been published in international forums and allow tackling with a lot of motivation the important milestones that this thesis poses. To sum up, the main milestones of the thesis are the following:

-Contextualization: the evaluation of the types of interaction that can be performed by pre-kindergarten children in multi-touch surfaces.

-Assistance: the definition of assistive techniques of interaction that allow the increase of effectiveness of pre-kindergarten actions with interactive surfaces.

-Communicability: the definition of effective mechanisms to communicate which actions are expected from the user through animated languages that allow children to be autonomous when using the multi-touch technology without the continuous supervision of adults.

-Adaptability: the definition of strategies that allow the adaptation of the Assistance and Communicability mechanisms to the specific skills of each user.

-Creativity: the construction and validation of environments that foster creativity and allow collaboration between users (pre-kindergarten children) to obtain in the future more adequate educational tools for these users.

\section{Acknowledgments}

Work supported by the MINECO (grants TIN2010-20488 and TIN2014-60077-R) and from GVA (ACIF/2015/075). 


\section{Part II}

\section{Usability}





\title{
Chapter 5
}

\section{Multi-touch Gestures for Pre-kindergarten Children}

Vicente Nacher ${ }^{1}$, Javier Jaen ${ }^{1}$, Elena Navarro ${ }^{2}$, Alejandro Catala ${ }^{1}$, Pascual Gonzalez ${ }^{2}$

${ }^{1}$ ISSI Group, Dpto. Sistemas Informáticos y Computación, Universitat Politècnica de València.

${ }^{2}$ LoUISE Research Group, Computing Systems Department, University of Castilla-La Mancha.

Published in International Journal of Human-Computer Studies 73, pp. 37-51. Elsevier (2015)

\begin{abstract}
The direct manipulation interaction style of multi-touch technology makes it the ideal mechanism for learning activities from pre-kindergarteners to adolescents. However, most commercial pre-kindergarten applications only support tap and drag operations. This paper investigates pre-kindergarteners' (2-3 years of age) ability to perform other gestures on multi-touch surfaces. We found that these infants could effectively perform additional gestures, such as one-finger rotation and two-finger scale up and down, just as well as basic gestures, despite gender and age differences. We also identified
\end{abstract}


Kindertivity: Usability and Communicability Strategies for Interactive Surfaces and PreKindergarten Children

cognitive and precision issues that may have an impact on the performance and feasibility of several types of interaction (double tap, long press, scale down and twofinger rotation) and propose a set of design guidelines to mitigate the associated problems and help designers envision effective interaction mechanisms for this challenging age range.

\subsection{Introduction}

Multi-touch technology has made great advances in recent decades, since its initial steps in the eighties, even before the adoption of graphical user interfaces, to its widespread acceptance today (Buxton, 2013). It now offers new sophisticated input and processing mechanisms that enable users to interact in a more natural and intuitive way (Smith et al., 2012). In fact, they are so natural and intuitive they have triggered a new approach to developing applications for children even younger than was previously thought possible. Rideout (Rideout, 2011) pointed out that even very young children (between 0 and 8) are frequent digital media users in the USA. For instance, her study showed that 38\% of them had used a Smartphone, iPad or similar device at least once. Among this group, 10\% were between 0 and 23 months and 39\% between 2 and 4 years old. The Horizon report (Johnson et al., 2012) supports this evidence and identifies mobile devices (smartphones and tablets) as one of the two emerging technologies suitable for children under two years old.

In order to provide users with natural and intuitive multi-touch systems, the direct manipulation interaction style and direct-touching are used. As Shneiderman and Plaisant (Shneiderman \& Plaisant, 2004) pointed out, there are three ideas behind the concept of direct manipulation: (1) visibility of objects and actions of interest; (2) replacement of typed commands by pointing-actions on objects of interest; and last but not least, (3) rapid, reversible and incremental actions that help to keep children engaged, give them control over the technology and avoid complex instructions. On the other hand, direct-touch, as Hourcade (Hourcade, 2007) stated, is preferred over mediated pointing devices like the mouse, as it provides a more direct way of selecting options on the screen. Moreover, as Couse and Chen (Couse \& Chen, 2010) stated, young children became totally engaged in their learning activities, even though they have to overcome certain technical difficulties.

This inherent ability of touch systems to engage children's attention is being widely exploited to promote learning activities from pre-kindergarteners to adolescents. For example, Mansor et al. (Mansor et al., 2009) have shown that tabletops can be operated by children as young as three and that there is no significant difference between learning in a real or virtual environment. Other studies (Sluis et al., 2004) (Khandelwal $\&$ Mazalek, 2007) and (Tyng et al., 2011a) have shown that this technology can be used by children between three and seven to learn to read, solve mathematical problems, develop a sense of space, etc. Other studies have demonstrated that the technology can also be used to promote collaboration between peers (Fleck et al., 2009; 
González et al., 2001; Rick \& Rogers, 2008; Rick et al., 2010) and to foster creativity (Catala et al., 2012; Helmes et al., 2009).

However, the increasing interest in multi-touch technology has not as yet given rise to studies on the design of multi-touch systems for the youngest age range, as Hourcade (Hourcade, 2007) has pointed out. Ingram et al. (Ingram et al., 2012) also concluded that although the set of multi-touch interactions that users and developers instinctively and unanimously agree upon is small (consisting of only one-finger touch for selection and one-finger drag for movement, and other fundamental tasks), the lack of standardized and universally accepted interactions makes the need for well-designed multi-touch interactions even more crucial.

In addition, such studies should be carefully designed as, according to Wolock et al. (Wolock et al., 2006), knowledge of children's developmental abilities is particularly important when designing software for the very young. This is especially relevant because, as these authors found, children between 18 and 30 months of age can use touch-screens under supervision. Therefore, studies that focus on pre-kindergarteners and older pre-school children must be carried out in order to provide them with technology specially tailored to their needs.

This paper thus addresses the fundamental question of the ability of prekindergarteners to perform gestures other than basic tap and drag operations on multitouch surfaces. We also aim to identify any issues that would require bespoke solutions specifically tailored to the needs of the very young.

The contributions of this work are manifold. The first contribution is a review of 100 commercial applications of multi-touch devices for pre-kindergarteners which reveals that most existing educational applications for these users only support tap and drag interactions. The second contribution is the experimental confirmation that prekindergarteners' abilities are by no means limited to these two basic actions, but they can also perform one-finger rotation and two-finger scale up and scale down, with equivalent success rates to those observed for the tap and drag gestures, despite gender and age differences. The third contribution is the identification of cognitive and precision issues that may have an impact on the performance and usability of several types of interaction. Finally, the fourth contribution is a set of design guidelines to mitigate the cognitive and precision-related issues identified in the course of this work, aimed at helping designers to envision effective interaction mechanisms for this challenging age range.

\subsection{Related work}

Interesting studies can be found in the literature that focus on the direct manipulation interaction style and have children as target population. For instance, Donker and Reitsma (Donker \& Reitsma, 2007) analyzed whether 6-7 year-old children made more errors while using a mouse to drag and drop than university students, and concluded 
Kindertivity: Usability and Communicability Strategies for Interactive Surfaces and PreKindergarten Children

that children were less skillful than adults, although most of their mistakes were due to the size of the destination area and the direction of the movement and not to the need to keep the mouse button pressed. The most frequent cause of these errors was their less developed fine motor abilities and eye-hand coordination. The shape of the mouse, which had been designed with adults in mind, was also identified as another cause of error.

Other interesting studies have examined the use of a tablet stylus for direct manipulation-interaction. For instance, Terra et al. (Terra, Brinkman, \& Heynderickx, 2009) and that the stylus had advantages over the alternatives.

Finally, still other studies focused on schoolchildren using both direct manipulation interaction and direct touching. For instance, Kharrufa et al. (Kharrufa, Leat, \& Olivier, 2010) presented a design process, grounded on both collaborative interaction and learning theories, for a collaborative learning application for 10 to 13 year-old children on tabletops. Their study shows the overwhelming superiority of tabletops for creating collaborative learning tools and gives some interesting guidelines for their development. Harris et al. (Harris et al., 2009) analyzed the differences between singletouch and multi-touch interaction in 7-10 year-old children's groups and found that although touch did not affect the interaction in terms of frequency or equity, it did influence their communication and encouraged them to talk to each other about their joint actions. Yu et al. (Yu, Zhang, Ren, Zhao, \& Zhu, 2010) assessed the design of the interaction aspect of touch screens in order to develop digital educational games. They tested the effectiveness of 5-6 year-olds in four game prototypes while dragging, clicking, rotating and zooming in and out. Their results indicated that pre-school children were not familiar with rotating and zooming and that they needed at least six minutes of training time. They also found that the main cause of the problem was the gap between the sensitivity of the device and the precision of the action required for the game. One of their negative findings was that more than half of the children rested their non-operational hand on the screen while using the interface.

Other studies have focused on pre-school children. For instance, Shoukry et al. (Shoukry, Sturm, \& G.H., 2012) defined a set of guidelines applicable to designing educational games for this group. Mansor et al. (Mansor et al., 2008) compared the interaction of 3-4 year-olds on a tabletop and in a physical setting. They concluded that children found it difficult to drag objects on the surface, mainly due to bad posture and suggested they should remain standing during these operations.

Other meaningful approaches, such as (Hourcade, Bullock-Rest, \& Hansen, 2011; Piper, O’Brien, Morris, \& Winograd, 2006; Rubio, Navarro, \& Montero, 2012; Weiss, Gal, Eden, Zancanaro, \& Telch, 2011), focused on the use of touch-screens by children with some type of cognitive disorder. They showed that it is possible to obtain significant results, especially in promoting collaboration and motivation, although these studies cannot be taken as a reference for normal children's behaviour with touch-screens. 
It can be seen from the above that touch-screen technology opens up a whole new world of possibilities for pre-school learning applications. This technology solves the problems inherent in other interaction devices, such as those involving mouse or keyboard, as it enables them to take advantage of both the direct manipulation interaction style and direct touch. Unfortunately, as far as we know, studies to date have only tested children over 3 years old, probably because age is a limiting factor; younger children do not have the verbal and cognitive skills to express their likes and preferences (Kremer, 2012), nor are they able to carry out tasks for long periods and are easily distracted (Egloff, 2004). However, according to Piaget (Piaget, 1973), children nowadays are in a preoperational stage from 2 years old onwards, i.e., they begin (1) to think in terms of images and symbols, and (2) develop symbolic play with imaginary objects, which means they could be candidates for multi-touch technology.

This led us to the main research question of this work: What multi-touch gestures are children between two and three years of age able to utilize? This paper provides an answer to this question by analyzing the most suitable gestures for pre-kindergarteners in terms of completion time and success.

\subsubsection{Commercial perspective on multi-touch technology}

The previous section carried out a review of some of the most interesting studies from a research perspective. However, it is also worth considering the commercial perspective of multi-touch technology and describing the two most popular currently available operating systems supporting multi-touch interaction: Android (Google, 2013) and iOS (Apple, 2013).

Regarding Android, we analysed 100 educational applications ${ }^{2}$, available to download from the Android AppStore, to determine the types of gestures used in these applications. The applications were randomly selected from the collection of preschool educational applications. Some of our most interesting conclusions are the following:

- There are three recurrent learning topics in these applications: numbers and math, thirty-five applications revolve around this topic; words and language, thirty-five applications focus on this topic; and colours and shapes; with twenty of the applications involving this topic. Some of the applications focus on other learning activities, such as types of animals or fruits.

- Regarding the learning methods (see Figure 19); fifty five applications use learning by reinforcement or repetitive methods, which consist of the repetition of the desired behaviour with a positive or negative reinforcement according to the learner's performance (Laird, 1985); forty-eight applications use receptive methods in which learners have to understand the content but do not discover anything new;

\footnotetext{
${ }^{2}$ Analysis of 100 Educational Applications http://dl.dropbox.com/u/52040030/RevisedGames.pdf
} 
Kindertivity: Usability and Communicability Strategies for Interactive Surfaces and PreKindergarten Children

and only five applications use active methods in which children have an active role in discovering the concepts and their relationships and adapting them to their cognitive schema (Michel, James, Iii, \& Varela, 2009). An example of the active method is the "Kids basic patterns" application, developed by Fun4Kids in which a shape appears following a pattern, a space and three options; the kids should choose the correct shape that fits the pattern.

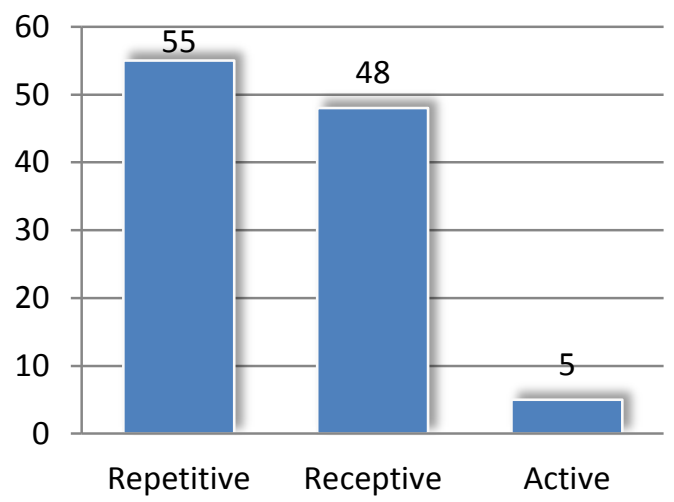

Figure 19. Learning methods

- Gestures: only three types of gestures are currently used (Figure 20). The onefinger tap is used in ninety-nine of the analysed applications, and the one-finger drag in fifty-six. Only three applications use an accelerometer for interaction. It is worth noting that only two of the analysed applications, "Animals Memory Game" and "KidMath", enable multiplayer mode (two players). These are also the only ones that allow the simultaneous interaction of two hands (one per participant).

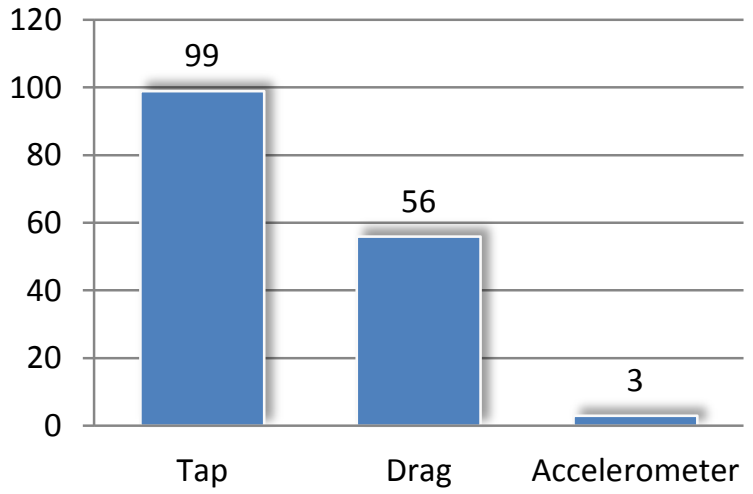

Figure 20. Supported interactions

A detailed analysis of iOS can be found in (Shuler, 2012). One of this study's most interesting conclusions is that $72 \%$ of the top-selling apps developed for iOS are aimed 
at children, with toddlers/pre-schoolers being the most popular age group (58\%). This category has also experienced the greatest growth (23\%), even higher than that of apps designed for adults, which shows the market importance of this target population and the potential to offer new solutions to these users. The report also highlights the need for a research agenda that guides developers and researchers towards creating effective, high-quality products.

Two main conclusions can be drawn from these results: firstly, there is clear confirmation of an important commercial trend in developing apps for children with touch-screen technology, mainly for learning activities. This highlights the need for empirical studies to help in the design of apps that adequately support children's development, as other authors have pointed out (Shuler, 2012). Secondly, but no less important, the results of the analysis of the Android Store (Figure 20) show that this technology is not being fully exploited for pre-school learning, as the supported gestures involved are too limited. Defining design guidelines that enable infants to take full advantage of multi-touch technology would make it possible to develop attractive new applications and eventually could also aid children's cognitive and motor development. We should highlight that designers and developers will make the final decision about the most appropriate gestures for their future apps according to other factors, such as cost or time to market, no matter what the results of this study are. Hopefully, the design guidelines proposed in this work will empower them to take a step forward in their developments.

\subsection{Experimental study}

The overall goal of our experimental study was to identify gestures suitable for prekindergarten children and to determine those best suited to future tablet applications targeted at children. Therefore, using the GQM (Goal Question Metric) template (Basili, Caldiera, \& Rombach, 1994), our goal can be defined as follows: analyze a set of multi-touch gestures for the purpose of evaluating their suitability from the viewpoint of usability in multi-touch technologies in the context of pre-kindergarten children. For this study we used children of both genders of between 2 and 3 years of age. We were interested in finding out whether certain gestures should be focused on a specific target gender, leading to further study on gender-based market segmentation. A specific age range was considered for the purposes of developmental issues. According to developmental theories, children are continuously developing and refining their motor skills between 2 and 7 years of age (Piaget, 1973). As we were more interested in exploring how gestures are learned and performed by very young children, we put the upper age limit at three years old. Gender and the 2 to 3 age group were thus the two main independent variables considered. Completion time and success rate were the two measured dependent variables for each task (tap, double tap, long pressed, drag, scale up, scale down, single rotation, one-finger rotation, two-finger 
Kindertivity: Usability and Communicability Strategies for Interactive Surfaces and PreKindergarten Children

rotation). Consequently, the hypotheses to be statistically tested, which were defined for each task performed (type of gesture), were formulated as follows:

- $\mathrm{H}_{1}$ : Completion time of task $k$ is not affected by gender.

- $\mathrm{H}_{2}$ : Completion time of task $k$ is not affected by age group.

- $\mathrm{H}_{3}$ : The degree of success for task $k$ is independent of gender.

- $\mathrm{H}_{4}$ : The degree of success for task $k$ is independent of age group.

We also defined another hypothesis related to the homogeneity of the success rates of the different tasks:

- $\mathrm{H}_{5}$ : The degree of success is independent of the task.

In order to test these hypotheses, we measured the manipulation time of each gesture as well as its success, with the ultimate goal of obtaining a set of guidelines specifically focused on designing touch-enabled applications for very young children

\subsubsection{Participants}

Thirty-two children aged between twenty-four and thirty-eight months took part in the experiment $($ Mean $(M)=31.34$, Standard Deviation $(S D)=4.24)$. The genders of the children were balanced, with 16 males and 16 females. Parental authorization was obtained before carrying out the study. The children were divided into two age groups: 24 to 30 months and 31 to 38 months, with 8 males and 8 females per group. We involved participants in the 2-to-3 age range from two Spanish nursery schools in order to explore how gestures are acquired and performed by children in the earliest stage of development. This age range corresponds to the first Spanish early education program for children between one and three. The starting age of our study is considered to be the start of the development of fine-grained motor skills (Piaget, 1973).

\subsubsection{Apparatus}

The interaction framework for the experiment was implemented in Java using JMonkeyEngine SDK v.3.0beta. The devices used for deployment and the experiment were a Motorola MZ601 and a Samsung Galaxy Note 10.1 tablet with Android 3.2 both with capacitive multi-touch screens.

\subsubsection{Procedure}

For each task, the children were given a 5-minute learning phase with an instructor. The experimental platform then asked them to perform the task without any assistance. They had to perform three repetitions of each gesture under specific conditions (see Section 5.4). When the gesture was completed successfully, the platform gave a positive audiovisual feedback. If the instructor saw that the participant did not carry out the task in a given time, it was marked as undone and the child went on to the next one. 
For each interaction, the system recorded the start time (seconds needed to go into action after the visual stimulus was shown), completion time, success (performed correctly or incorrectly), and the number of contacts with the surface (in order to know in an unsuccessful action whether the user had made any attempt to interact). A qualitative analysis was also carried out from the notes taken by an external observer during the experimental sessions.

\subsection{Tasks}

\subsubsection{Task 1: Tap}

A static image of an animal appears in a random position on the screen (see Figure 21). Participants are requested to tap on the target image in order to pass the test.

\subsubsection{Task 2: Double Tap}

A static image of an animal appears in a random position on the screen (see Figure 21). Participants are requested to double tap on the target image with one finger in order to pass the test. The task will succeed when the participants perform two taps in under 300 milliseconds, which is Android's default time interval for this gesture.

\subsubsection{Task 3: Long Pressed}

A static image of an animal appears in a random position on the screen (see Figure 21). Participants are requested to carry out a long pressed gesture on the target image until the target disappears. The task will succeed when the participants put their finger on the target image and hold it for at least 500 milliseconds, which is Android's default time interval for this gesture.

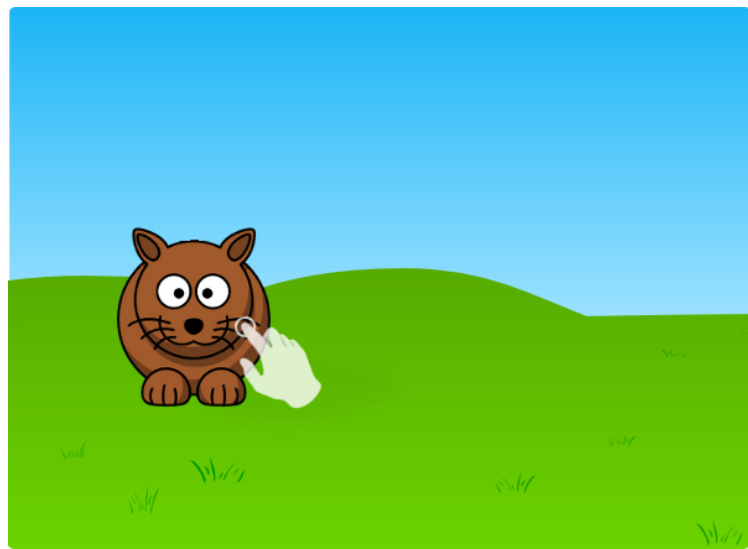

Figure 21. Example of a simple tap, double tap or long pressed test 
Kindertivity: Usability and Communicability Strategies for Interactive Surfaces and PreKindergarten Children

\subsubsection{Task 4: Drag}

A static image of an animal appears in a random position on the screen and the same (reference) image appears in a white profile in another random position, always at a distance of 378 pixels so as to be able to compare execution times among the different subjects (Figure 22). The random position of the reference image is subject to some geometric restrictions, to make sure that it is completely visible on the surface. Participants are requested to drag the target to the reference image with one finger. The task will succeed when the target image reaches the location of the reference image with a precision of less than 10 pixels on each $\mathrm{X}$ and $\mathrm{Y}$ axis. It is not necessary for the subject to lift his/her hand to reach success.

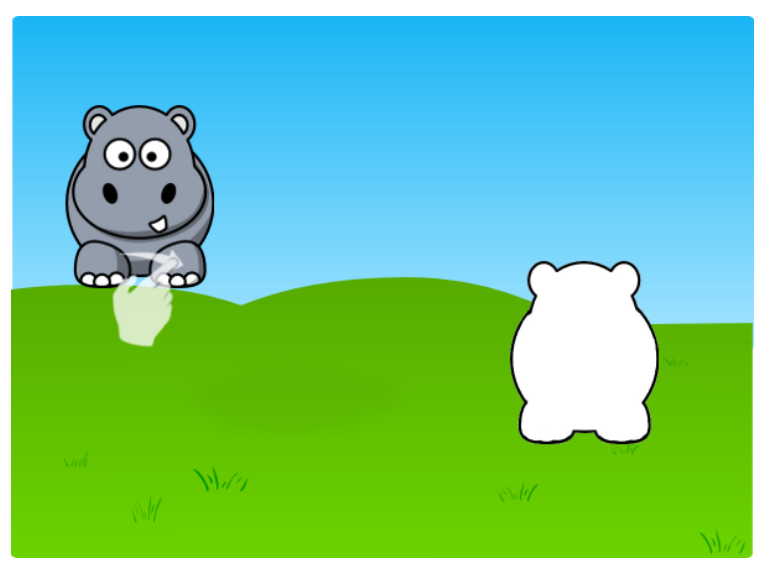

Figure 22. Example of a drag test

\subsubsection{Task 5: Scale up}

A static image of an animal appears in the center of the screen within a similar but 1.5 times larger reference shape (see Figure 23-a). Participants are requested to scale up the target image to the size of the reference shape. This can be done by expanding the distance between two fingers of either one hand or two hands. The fingers do not have to be in contact with the reference image and the scaling factor applied is the incremental value returned by the JMonkeyEngine runtime for this gesture. If more than two contacts are made on the surface, JMonkeyEngine considers only the two most recent ones for communicating scaling events. The task will succeed when the target image reaches the size of the reference image, not being necessary for the subject to lift his/her hands when the size of the target image is reached.

\subsubsection{Task 6: Scale down}

A static image of an animal appears in the center of the screen superimposed on a similar reference shape half its size (see Figure 23-b). Participants are requested to scale down the target image by making the target object shrink until it reaches the size 
of the reference image with two fingers of either one or two hands. The task will succeed when the target image reaches the size of the reference image as in the previous case.

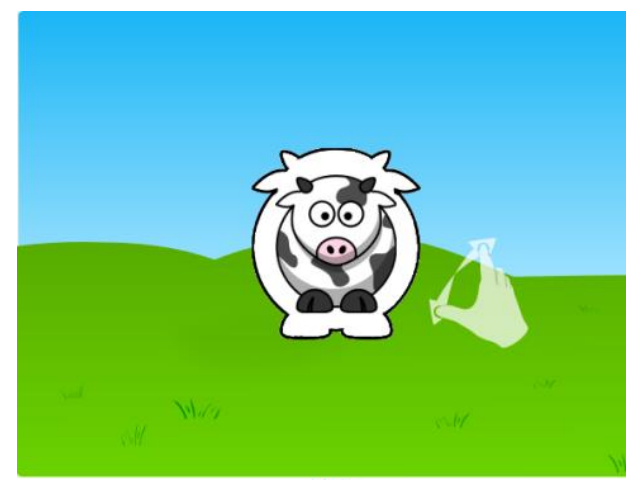

(a)

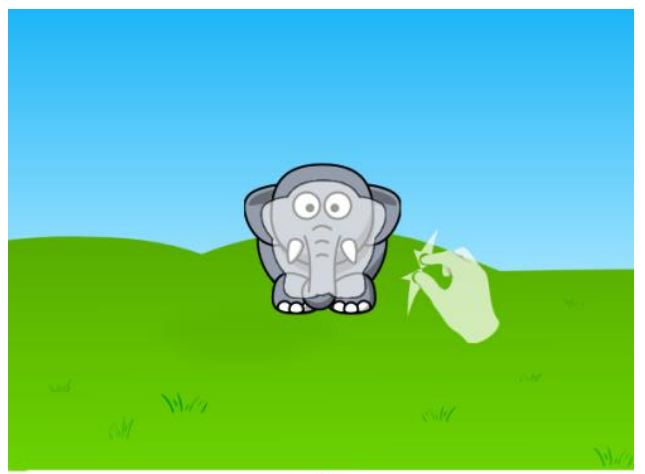

(b)

Figure 23. Example of a scale test: (a) scale up (b) scale down

\subsubsection{Task 7: One-finger rotation}

A static image of an animal appears in the center of the screen in front of a blank profile of the same image in a different orientation. Rotation is always clockwise to a fixed position so as to be able to compare interaction execution times among subjects (see Figure 24). Participants are requested to rotate the target image to the position of the reference image by dragging one finger around the center of the target image. Pressure can be applied on the target image itself or anywhere around it. The task will succeed when the target image reaches an angle larger than the specified goal which is automatically detected by the system to produce the positive audiovisual feedback.

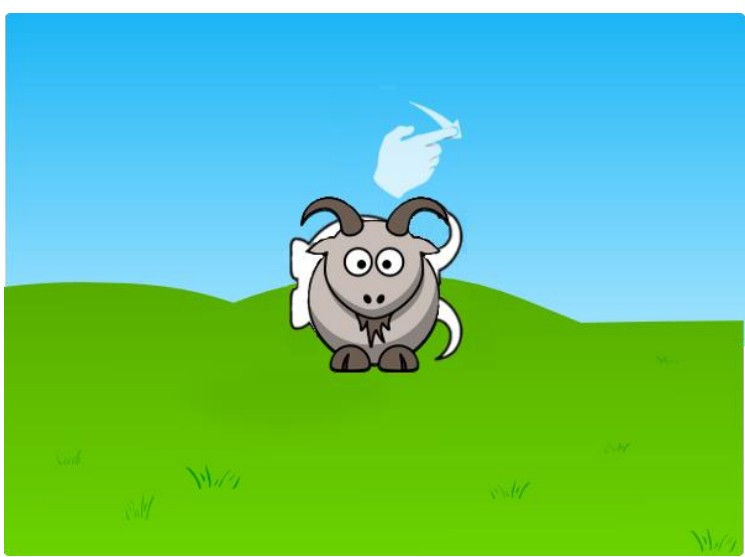

Figure 24. Example of a One-Finger rotation test 
Kindertivity: Usability and Communicability Strategies for Interactive Surfaces and PreKindergarten Children

\subsubsection{Task 8: Two-finger rotation}

A static image of an animal appears in the center of the screen in front of a blank profile of the same image but always rotated clockwise to a fixed position, so as to be able to compare interaction execution times among subjects (see Figure 25). Participants are requested to rotate the target image with two fingers until it reaches the position of the reference image. The task will succeed when the target image reaches the orientation of the reference image. The system detects this situation and produces the visual reward, it not being necessary for the subject to lift his/her hands.

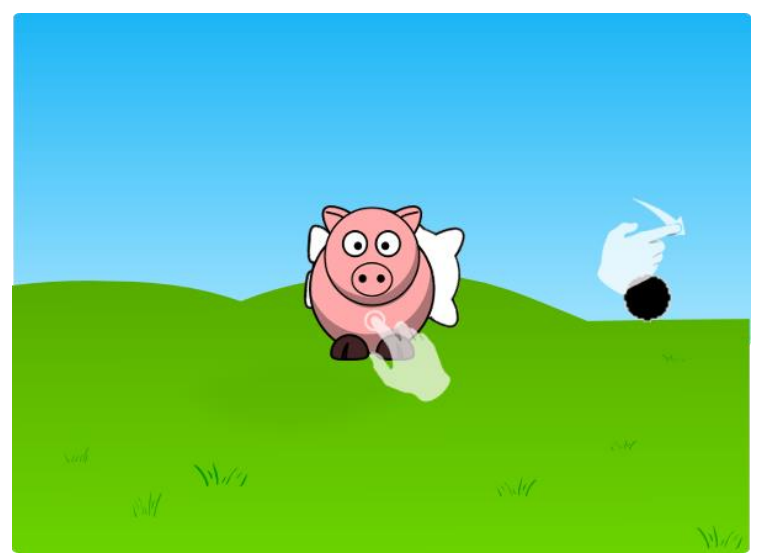

Figure 25. Example of a Two-Finger rotation test

The children were initially asked to use two fingers in pilot tests, although this was soon discarded because they were found to touch the screen with the whole hand, not just the fingers, producing multiple contacts on the surface. As the task thus became unfeasible, this led us to ask the children to touch and hold one finger on the target image, at which point a black spot appeared on the right of the screen. They then had to rotate one finger around the black spot until the image reached the orientation of the reference shape. If the finger was not kept on the target before the correct position was reached, the black point disappeared and the rotation was disabled until the participant again touched the target object.

\subsection{Results}

This section presents the results of the experimental tests, according to each of the analyzed independent variables. Completion time is dealt with in Section 5.5.1 and the success rate in Section 5.5.2. The tasks are compared in Section 5.5.3 and the qualitative results are presented in Section 5.5.4. 


\subsubsection{Completion time}

The three trials carried out by each participant were combined to perform the subsequent analysis. The average of each subject's successful tasks is used to obtain the average completion time value per task and user. If the test is not performed successfully it is not included in the completion time analysis, resulting in different statistical degrees of freedom for each task. Mean completion time for each task is presented in Table 10 and Table 11 (see Appendix A) by age group and gender. The results are also shown graphically in Figure 26 and Figure 27.

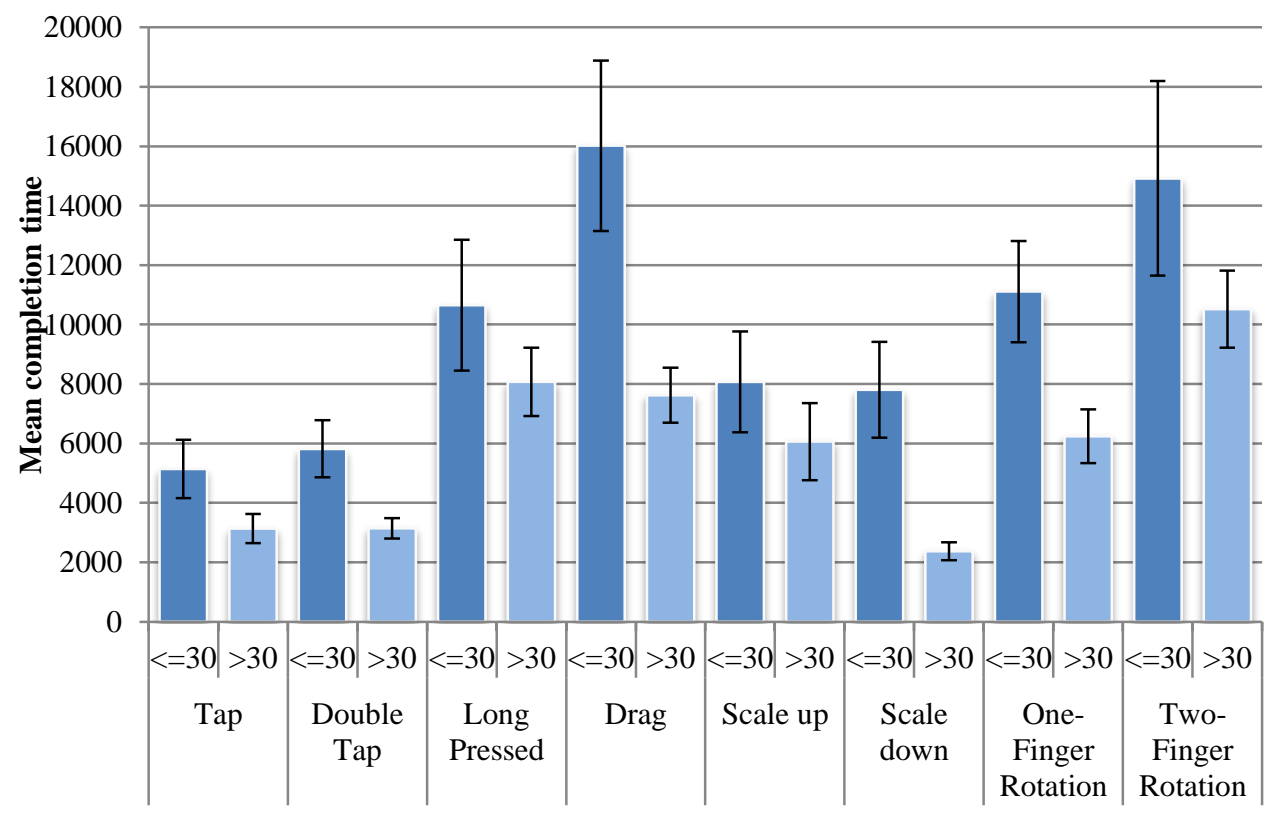

Figure 26. Mean completion time by task and by age group. Error bars reflect standard error.

Table 2 shows the tested hypotheses in relation to completion time. The application of a two-way between-subject ANOVA with the independent variables gender and age group and dependent variable completion time demonstrated that it is not significantly influenced by gender (see Table 3 ), so that Hypothesis $\mathrm{H}_{1}$ cannot be rejected for any of the tasks. The analysis also showed that completion time is not significantly influenced by the interaction of the gender and age group factors, meaning that $\mathrm{H}_{12}$ cannot be rejected either (Table 3 ). 
Kindertivity: Usability and Communicability Strategies for Interactive Surfaces and PreKindergarten Children

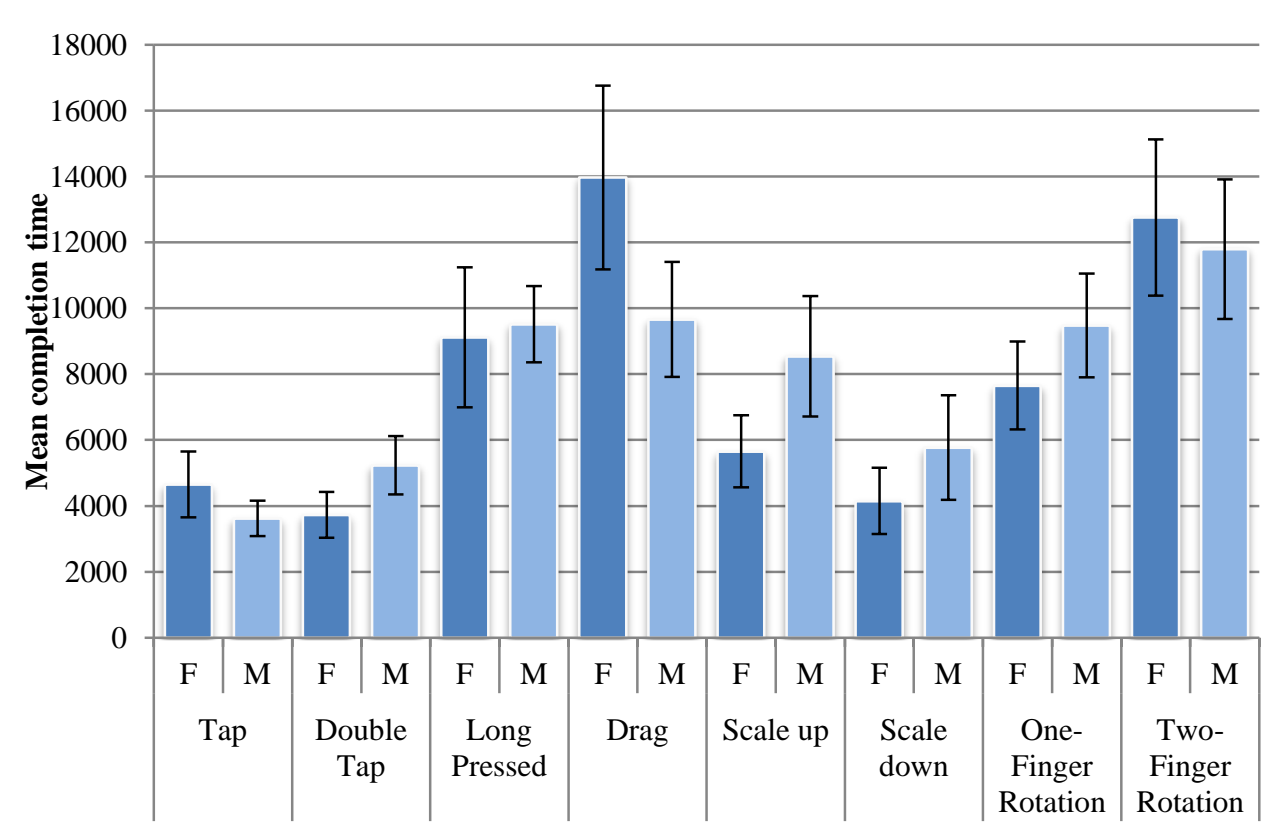

Figure 27. Mean completion time by task and by gender (F: Female, M: Male)

Table 2. Main features of the experiment regarding completion time

\begin{tabular}{ll}
\hline Null- & $\mathrm{H}_{1}:$ Completion time of task $k$ is not affected by gender. $\mathrm{H}_{1}: \neg \mathrm{H}_{1}$ \\
Hypotheses & $\begin{array}{l}\mathrm{H}_{2}: \text { Completion time of task } k \text { is not affected by age group. } \mathrm{H}_{2}: \neg \mathrm{H}_{2} \\
\mathrm{H}_{12}: \text { Completion time of task } k \text { is not affected by the interaction of gender and age } \\
\text { group. } \mathrm{H}_{12}: \neg \mathrm{H}_{12}\end{array}$ \\
\hline $\begin{array}{l}\text { Dependent } \\
\text { Variable }\end{array}$ & Completion time of task $k$ performed by the children \\
\hline $\begin{array}{l}\text { Independent } \\
\text { Variables }\end{array}$ & The gender and age range to which the children belong \\
\hline Location & Polytechnic University of Valencia (Valencia, Spain) \\
\hline Date & March 2013 \\
\hline Subjects & 32 pre-kindergarteners (16 males and 16 females) \\
\hline
\end{tabular}

The analysis also demonstrated that the double tap, drag, scale down and one-finger rotation tasks are significantly influenced by the age group factor (p-value $<0.05)$, so that Hypothesis $\mathrm{H}_{2}$ is rejected for these tasks. The participants in the second age group ( 31 to 38 months) performed these gestures faster than those in the first group (24 to 30 months). 
Multi-touch gestures for pre-kindergarten children

Table 3. F-Statistics of the completion time analysis

\begin{tabular}{l|c|c|l|l|l|c|l}
\hline \multirow{2}{*}{ Task } & \multirow{2}{*}{ DoF } & \multicolumn{2}{c|}{ Gender } & \multicolumn{2}{c|}{ Age group } & \multicolumn{2}{c}{ Gender*Age } \\
\cline { 3 - 8 } & & F & p-value & F & p-value & F & p-value \\
\hline Tap & $(1.32)$ & 0.963 & 0.335 & 3.599 & 0.068 & 3.143 & 0.087 \\
\hline Double Tap & $(1.24)$ & 2.395 & 0.137 & 7.568 & 0.012 & 1.931 & 0.180 \\
\hline Long Pressed & $(1.23)$ & 0.020 & 0.888 & 1.018 & 0.326 & 2.319 & 0.144 \\
\hline Drag & $(1.32)$ & 2.179 & 0.151 & 8.246 & 0.008 & 1.778 & 0.193 \\
\hline Scale Up & $(1.30)$ & 2.528 & 0.124 & 1.414 & 0.245 & 1.755 & 0.197 \\
\hline Scale Down & $(1.30)$ & 2.018 & 0.167 & 14.148 & 0.001 & 1.417 & 0.245 \\
\hline One-Finger Rotation & $(1.31)$ & 0.715 & 0.405 & 6.250 & 0.019 & 0.185 & 0.671 \\
\hline Two-Finger Rotation & $(1.20)$ & 0.098 & 0.758 & 1.818 & 0.196 & 0.009 & 0.925 \\
\hline
\end{tabular}

On the other hand the tap, long pressed, scale up and two-finger rotation tasks are not significantly influenced by the age group factor and, consequently, $\mathrm{H}_{2}$ cannot be rejected for them. Nevertheless, on average, these gestures are performed faster by the second age group, as can be seen in Figure 26. The results therefore show that, in the analyzed age range, the older participants are faster at performing the tasks.

\subsubsection{Success}

The three trials carried out by each participant were also combined. If a participant performed successfully either zero or one tests in a specific task, he (she) was considered incapable of performing it, whereas if they successfully performed two or three tests in a specific task, they were considered capable of doing it as they actually show their ability to consistently reproduce the gesture several times. According to this codification, the degree of success in each task can be expressed as a percentage, as shown in Table 12 and Table 13 (see Appendix A) by age group and gender and graphically in Figure 28 and Figure 29.

Table 4 shows the tested hypotheses. Pearson's chi-square tests were conducted on each gesture in order to determine the independence of success from two qualitative factors (gender and age group).

The tests showed that there is no empirical evidence to say that degree of success and gender are not independent (see Table 5). Hence, the hypothesis $\mathrm{H} 3$ cannot be rejected and the degree of success does not therefore have a significant relationship with gender. Furthermore, the analysis also showed that H4, the hypothesis on the independence of degree of success and age group cannot be rejected, which means that the degree of success does not significantly differ by age group. The analysis also showed that there is no empirical evidence to say that degree of success is not independent of the joint gender *age group and $\mathrm{H}_{34}$ cannot be rejected. The success rate by task for each age group is shown in Figure 28 and for each gender in Figure 29, in which it can be seen to be similar for both age groups and genders. 
Kindertivity: Usability and Communicability Strategies for Interactive Surfaces and PreKindergarten Children

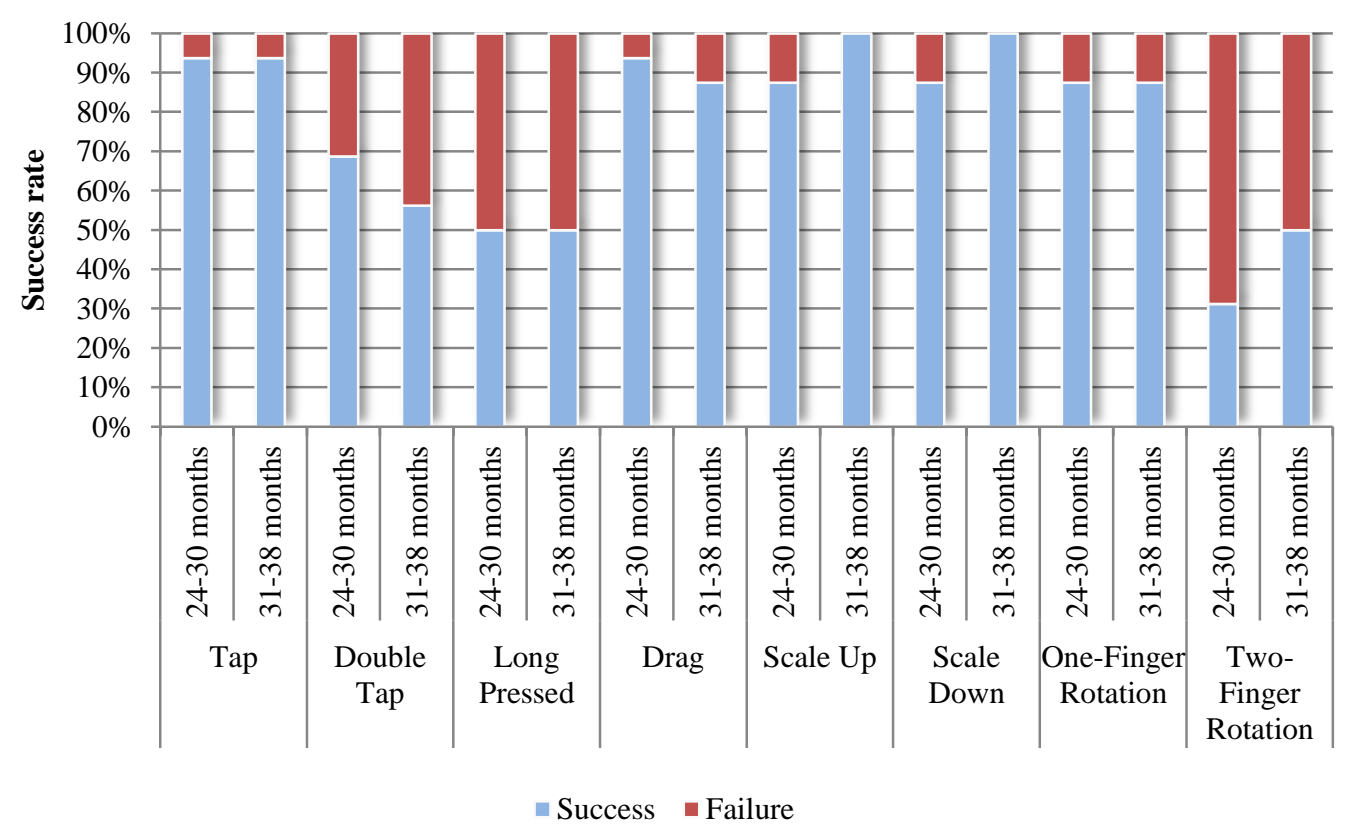

Figure 28. Success rate by task and age group

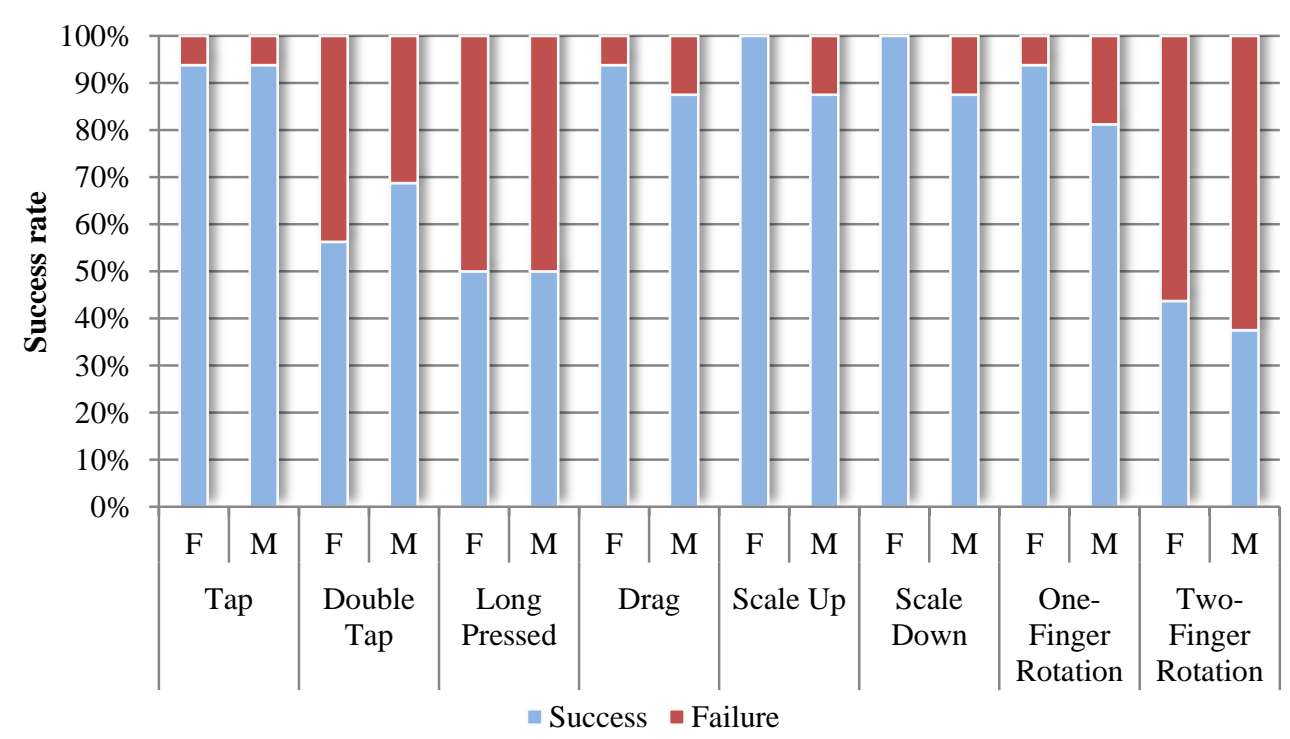

Figure 29. Success rate by task and gender (F: Female, M: Male) 
Table 4. Main features of the experiment regarding degree of success

\begin{tabular}{ll}
\hline Null- & $\mathrm{H}_{3}:$ The degree of success for task $\mathrm{k}$ is independent of gender. $\mathrm{H}_{3}: \neg \mathrm{H}_{3}$ \\
Hypotheses & $\begin{array}{l}\mathrm{H}_{4}: \text { The degree of success for task } \mathrm{k} \text { is independent of age group. } \mathrm{H}_{4}: \neg \mathrm{H}_{4} \\
\mathrm{H}_{34}: \text { The degree of success for task } \mathrm{k} \text { is independent of the joint gender } * \text { age } \\
\text { group. } \mathrm{H}_{34}: \neg \mathrm{H}_{34}\end{array}$ \\
\hline $\begin{array}{l}\text { Dependent } \\
\text { Variable }\end{array}$ & Success of task $k$ performed by the children \\
\hline $\begin{array}{l}\text { Independent } \\
\text { Variables }\end{array}$ & The gender and age range to which the children belong \\
\hline Location & Valencia, Spain \\
\hline Date & March 2013 \\
\hline Subjects & 32 pre-kindergarteners (16 males and 16 females) \\
\hline
\end{tabular}

Table 5. Statistics of Pearson's chi-square test

\begin{tabular}{|c|c|c|c|c|c|c|c|c|}
\hline \multirow{2}{*}{ Task } & \multirow{2}{*}{ DoF } & \multicolumn{2}{|c|}{ Gender } & \multicolumn{2}{|c|}{ Age group } & \multirow{2}{*}{ DoF } & \multicolumn{2}{|c|}{ Gender*Age } \\
\hline & & $\chi^{2}$ & p-value & $\chi^{2}$ & p-value & & $\chi^{2}$ & p-value \\
\hline Tap & 1 & 0.00 & 1.000 & 0.00 & 1.000 & 3 & 2.11 & 0.545 \\
\hline Double Tap & 1 & 0.53 & 0.465 & 0.53 & 0.465 & 3 & 1.06 & 0.785 \\
\hline Long Pressed & 1 & 0.00 & 1.000 & 0.00 & 1.000 & 3 & 0.00 & 1.000 \\
\hline Drag & 1 & 0.36 & 0.544 & 0.36 & 0.544 & 3 & 1.10 & 0.776 \\
\hline Scale Up & 1 & 2.13 & 0.144 & 2.13 & 0.144 & 3 & 6.40 & 0.094 \\
\hline Scale Down & 1 & 2.13 & 0.144 & 2.13 & 0.144 & 3 & 6.40 & 0.094 \\
\hline One-Finger Rotation & 1 & 1.14 & 0.285 & 0.00 & 1.000 & 3 & 2.28 & 0.515 \\
\hline Two-Finger Rotation & 1 & 0.13 & 0.719 & 1.16 & 0.280 & 3 & 1.42 & 0.700 \\
\hline
\end{tabular}

Figure 30 shows a histogram of the number of users able to perform a given number $k$ of tasks successfully ( $k$ ranging between 0 and 8 ). On one hand, if we consider the worst performers, the data reveal that there are no users who perform six or more tasks erroneously, i.e., even the worst users are able to perform at least 3 tasks successfully. These subjects are consistently able to perform the tap, scale up and scale down gestures. On the other hand, if we look at the best performers we observe that $75 \%$ of the evaluated children are able to perform six or more gestures correctly. These skilled children fail consistently when performing the two-finger rotation and long pressed tasks. These observations will be discussed later with respect to cognitive and motor factors in Sections 5.5.4 and 5.6.3. 
Kindertivity: Usability and Communicability Strategies for Interactive Surfaces and PreKindergarten Children

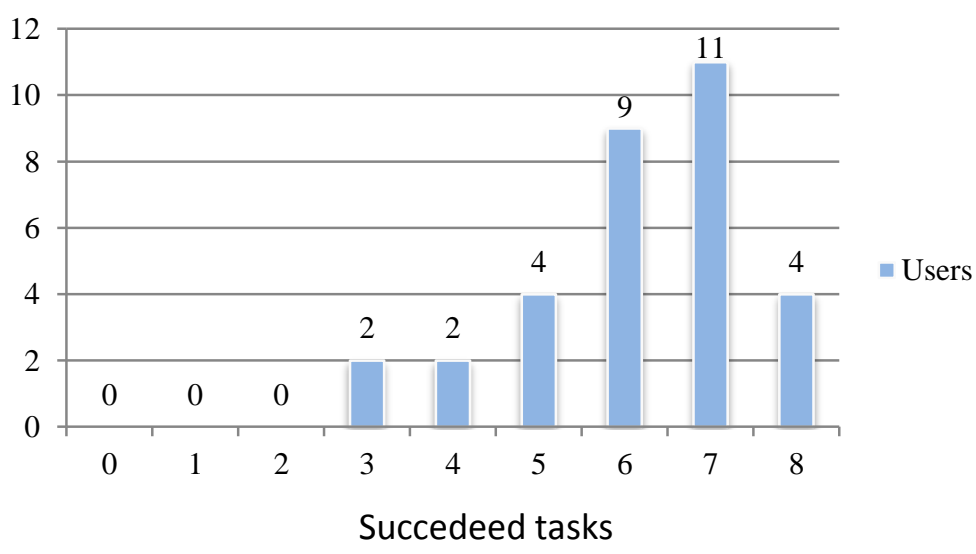

Figure 30. Users grouped by number of successfully performed tasks

\subsubsection{Comparing tasks}

The success rate for each task is shown in Figure 31, in which it can be seen that not all the tasks are equally feasible.

A classification of the evaluated gestures was carried out: Tap and Drag are already implemented in commercial applications; Scale up/down and one finger rotation are not implemented in most commercial applications, despite the considerable success rate achieved by the pre-school children in our tests; Double Tap and Long Pressed are classified as eligible for implementation in future apps, subject to certain guidelines (see section 5.6.3); and Two-Finger Rotation would have to be discarded or remodeled to be included in learning applications for pre-kindergarteners. 


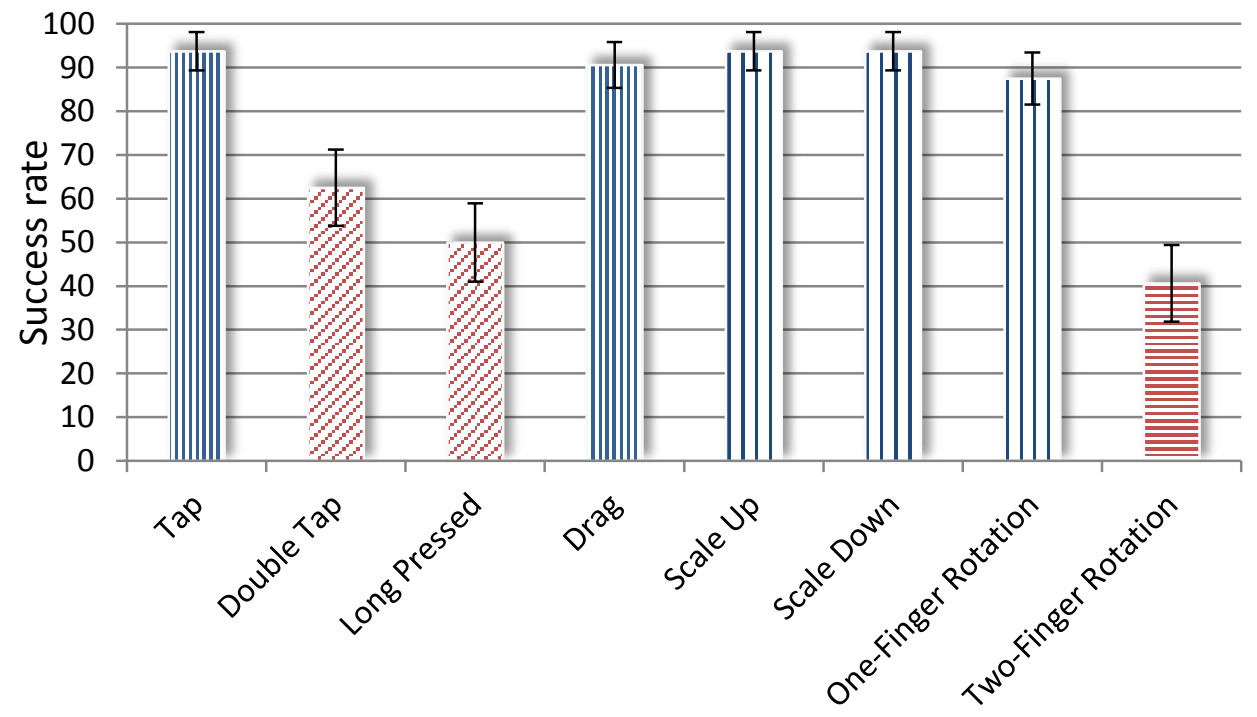

Figure 31. Success rate by task

The null hypothesis shown in Table 6 was formulated and a pair-wise task comparison was conducted to test whether degree of success was independent of task.

Table 6. Main features success in the experimental tests

\begin{tabular}{ll}
\hline Null-Hypotheses & $\mathrm{H}_{5}$ : The degree of success is independent of the Task. $\mathrm{H}_{5}: \neg \mathrm{H}_{5}$ \\
\hline Dependent Variable & Success of gestures performed by the children \\
\hline Independent Variables & The task performed by the children \\
\hline Location & Valencia, Spain \\
\hline Date & March 2013 \\
\hline Subjects & 32 pre-kindergarteners (16 males and 16 females) \\
\hline
\end{tabular}

Pearson's chi-square test of independence was again used to analyze the degree of success of the task. Table 7 shows the results of the statistical analysis. Each cell contains the significance obtained from the analysis of each pair of tasks $\left(^{*}, \mathrm{p}<0.05\right.$; **, $\mathrm{p}<0.001)$.

In accordance with Table 7, $\mathrm{H} 5$ is rejected due to the fact that there are gestures with statistically different success rates. According to the statistical tests, a first category of gestures (tap, drag, scale up, scale down and one-finger rotation) is identified in which there are no statistically significant differences in terms of success rate, all having a success rate close to 90 per cent. 
Kindertivity: Usability and Communicability Strategies for Interactive Surfaces and PreKindergarten Children

On the other hand, double tap, long pressed and two-finger rotation have statistically significant differences with the gestures in the first group. These are the most problematic gestures with the lowest success rate.

The variance in completion time of each task was then analyzed, as shown in Table 8 . Table 9 shows the Levene's tests for the homogeneity of variances contrasting gestures. Each cell contains the significance of a combination of two tasks. In this case $\chi^{2}($ DoF $($ Task X, Task Y $)=\operatorname{DoF}($ Task X) + DoF(Task Y)-2 (see Table 3 for the DoF values of each task). Given the large number of comparisons (a family of $\mathrm{m}=28$ hypotheses) we applied a Bonferroni correction that establishes statistical significance at $\mathrm{p}<0.05 / 28=0.002$.

Table 7. Task comparison by success with Pearson's chi-square test of independence $\chi^{2}$ $(\mathrm{DoF}=1, \mathrm{~N}=32)$.

\begin{tabular}{|c|c|c|c|c|c|c|c|c|}
\hline Success & Tap & $\begin{array}{r}\text { Double } \\
\text { Tap }\end{array}$ & $\begin{array}{r}\text { Long } \\
\text { Pressed }\end{array}$ & Drag & Scale Up & $\begin{array}{r}\text { Scale } \\
\text { Down }\end{array}$ & $\begin{array}{l}\text { 1-Finger } \\
\text { Rotation }\end{array}$ & $\begin{array}{l}\text { 2-Finger } \\
\text { Rotation }\end{array}$ \\
\hline Tap & & $0.002 *$ & $\begin{array}{l}0.000 \\
* *\end{array}$ & 0.641 & 1.000 & 1.000 & 0.391 & $\begin{array}{l}0.000 \\
* *\end{array}$ \\
\hline $\begin{array}{l}\text { Double } \\
\text { Tap }\end{array}$ & & & 0.313 & $0.008 *$ & $0.002 *$ & $0.002 *$ & $0.021 *$ & 0.080 \\
\hline $\begin{array}{l}\text { Long } \\
\text { Pressed }\end{array}$ & & & & $\begin{array}{l}0.000 \\
* *\end{array}$ & $\begin{array}{l}0.000 \\
* *\end{array}$ & $\begin{array}{l}0.000 \\
* *\end{array}$ & $0.001 *$ & 0.451 \\
\hline Drag & & & & & 0.641 & 0.641 & 0.689 & $\begin{array}{l}0.000 \\
* *\end{array}$ \\
\hline $\begin{array}{l}\text { Scale } \\
\text { Up }\end{array}$ & & & & & & 1.000 & 0.391 & $\begin{array}{l}0.000 \\
* *\end{array}$ \\
\hline $\begin{array}{l}\text { Scale } \\
\text { Down }\end{array}$ & & & & & & & 0.391 & $\begin{array}{l}0.000 \\
* *\end{array}$ \\
\hline $\begin{array}{l}\text { 1-Finger } \\
\text { Rot. }\end{array}$ & & & & & & & & $\begin{array}{l}0.000 \\
* *\end{array}$ \\
\hline
\end{tabular}

Table 8. Variance of completion time (in seconds) by task.

\begin{tabular}{l|l|l}
\hline \multicolumn{2}{|l|}{} & Variance \\
\hline \multirow{4}{*}{ Task } & Tap & 10.29 \\
\cline { 2 - 3 } & Double Tap & 7.84 \\
\cline { 2 - 3 } & Long Pressed & 33.83 \\
\cline { 2 - 3 } & Drag & 88.69 \\
\cline { 2 - 3 } & Scale Up & 33.00 \\
\cline { 2 - 3 } & Scale Down & 24.74 \\
\cline { 2 - 3 } & One-Finger Rotation & 33.07 \\
\cline { 2 - 3 } & Two-Finger Rotation & 48.21 \\
\hline
\end{tabular}


The results obtained show that the first group of tasks can be established which includes tap, double tap, long pressed and scale down which have the lowest level of variance, i.e. there is homogeneity in the results in terms of completion time for these gestures.

Scale up, one-finger rotation and two-finger rotation compose the second group, with a higher level of variance, which implies that their data is more disperse and with some differences between the subjects when performing the task.

Lastly, drag composes the third group, with the highest level of variance, which means a high rate of dispersion, implying wide variations in completion times caused by issues that will be discussed in the next section.

Table 9. Results of Levene's test

\begin{tabular}{l|l|l|l|l|l|l|l|l}
\hline Success & Tap & $\begin{array}{r}\text { Double } \\
\text { Tap }\end{array}$ & $\begin{array}{r}\text { Long } \\
\text { Pressed }\end{array}$ & Drag & Scale Up & $\begin{array}{r}\text { Scale } \\
\text { Down }\end{array}$ & $\begin{array}{r}\text { 1-Finger } \\
\text { Rotation }\end{array}$ & $\begin{array}{l}\text { 2-Finger } \\
\text { Rotation }\end{array}$ \\
\hline Tap & & 0.386 & 0.026 & $\begin{array}{l}0.000 \\
* *\end{array}$ & $0.001 *$ & 0.049 & $0.001 *$ & $0.002 *$ \\
\hline $\begin{array}{l}\text { Double Tap } \\
\begin{array}{l}\text { Long } \\
\text { Pressed }\end{array}\end{array}$ & & 0.013 & $\begin{array}{l}0.000 \\
* *\end{array}$ & $0.000 * *$ & 0.018 & $\begin{array}{l}0.000 \\
* *\end{array}$ & $0.001 *$ \\
\hline Drag & & & & 0.053 & 0.627 & 0.611 & 0.627 & 0.397 \\
\hline Scale Up & & & & & 0.059 & 0.009 & 0.056 & 0.257 \\
\hline $\begin{array}{l}\text { Scale Down } \\
\text { 1-Finger } \\
\text { Rot. }\end{array}$ & & & & & & 0.239 & 0.998 & 0.571 \\
\hline
\end{tabular}

\subsubsection{Qualitative results}

In addition to the automatic data logging that was performed to measure completion times and degree of success, an external observer gathered valuable information regarding the behavior of children during the experiments. These observations revealed different problems that will now be described.

We observed several precision problems due to the subjects being in the early development phase of fine motor skills. Firstly, we observed precision problems when asking pre-kindergarteners to tap an element with one finger and hold it for a given amount of time (long-pressed). In this specific case, the children had an entry precision problem that prevented them from keeping their finger in a fixed position at the start of the interaction. Instead, they performed a drag around the entry point, where the finger then remained pressed. This makes the system misinterpret the initial contact, because the start of a drag gesture prevents it from identifying a long-pressed gesture, no matter how long the finger is kept pressed. Secondly, we observed problems associated with estimating the speed at which a given interaction was supposed to take place. In our 
Kindertivity: Usability and Communicability Strategies for Interactive Surfaces and PreKindergarten Children

particular case, this was revealed with the double tap gesture when some children were unable to perform the second tap as quickly as expected by the underlying gesture detection middleware.

In addition to the above precision issues, there are even more critical issues that were observed related to the cognitive complexity required by some of the interactions that have been studied in this work.

In the first place, a recurrent situation was the cognitive complexity associated with the process of counting. This was especially apparent in the double tap interaction, when several children were unable to stop the interaction after tapping twice on a given object. Instead, they would tap repeatedly on the target figure and get upset because the system was not rewarding them after performing a great number of taps. It could be argued that it is not clear whether the children were not aware of having made more than two taps or whether it was a motor inhibition problem. In this respect, a postexperimental discussion was carried out with the children's teachers and it became clear that there were several reasons. Some children, while being able to count, had motor-inhibition problems, whereas others were unable to count the number of required events.

Another cognitive-related problem was revealed when the children were asked to perform a two-finger rotation. We have to remember here that the standard two-finger rotation was not appropriate for these users, due to an ergonomic problem when they placed both hands on the surface. Consequently, we designed a two-finger rotation mechanism that required one finger to be kept on the object to be rotated (long pressed) and another to move around a stationary point displayed on the screen (one finger rotation). However, we observed that this combined interaction presented a cognitive challenge. Most of the children behaved in the opposite way by keeping a finger on the stationary rotation point and another rotating around the item that they had got used to rotating.

Finally, as has been pointed out in the statistical analysis of the quantitative data, age was a significant factor in the completion time of the scale down task but, surprisingly, this was not the case for scale up (see Table 3). The analysis of the notes on these tasks taken by an external observer revealed that when scaling up the children started from an initial situation in which their two fingers were close together, so that it was then easy for them to separate their fingers while using all the available space (see Figure 32 left). However, when performing the scale down task, the youngest children (24-30 months) usually started the interaction with their fingers in the same position as before (close together on the surface) and so were forced to continuously repeat the following sequence: move fingers together, take fingers off surface, put fingers on surface but close to each other. This situation did not arise with children in the second age group (31-38 months), who were able to estimate the initial distance between their fingers on the surface and could perform the task with one, or at most two, scale down operations. 


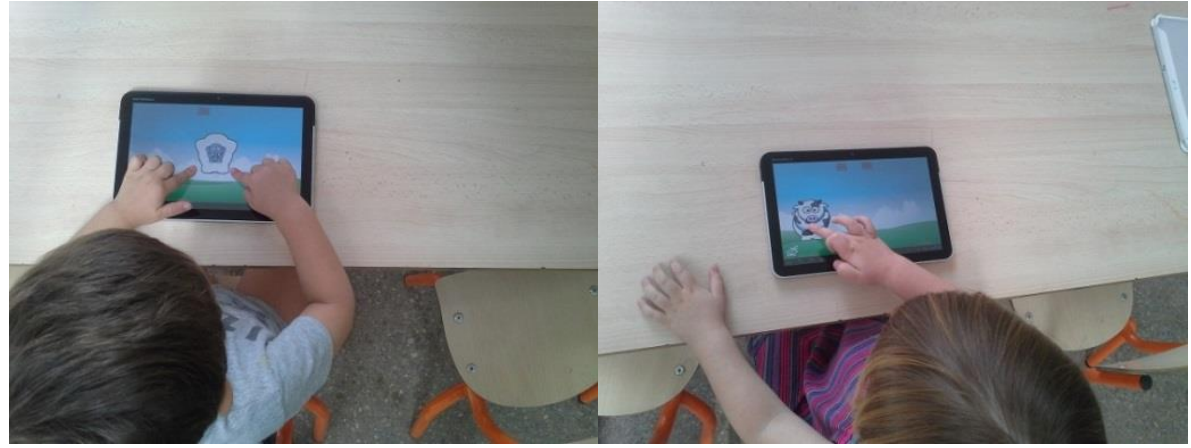

Figure 32. Examples of actual scale (left) and drag (right) gestures

\subsection{Discussion}

\subsubsection{Debunking myths}

Based on the above results, the answer to the fundamental question, "Are prekindergarten children ready for multi-touch technology?" is definitely affirmative, provided certain issues are dealt with. Regarding the question "What multi-touch gestures are children between two and three years of age able to use?", we found that the general belief that pre-school children are only capable of performing tap and drag interactions is really no more than a myth. Our study found similar levels of success for interactions such as one-finger rotation and two-finger scale up \& down, as for tap and drag, already mainstream in existing applications. Consequently, interaction designers have an opportunity to broaden the scope of their interfaces when creating future applications. Current applications for pre-kindergarten children might be missing the opportunity to provide richer gestures within children's abilities, and could be using a gesture that is notoriously difficult for them (e.g., double tap or two-finger rotation). Therefore, these results show there is no justification for the current situation of most commercial applications for pre-kindergarteners which only support two gestures: tap and drag (present in $99 \%$ and $56 \%$ of the analysed applications respectively).

The quantitative results also show that there are still challenging gestures for prekindergarteners (double tap, long press and two-finger rotation) with success rates ranging between $40 \%$ and $60 \%$. These gestures will be discussed below in the context of the interaction aids or design guidelines that application designers will have to take into account if these touch interactions are included in future applications.

\subsubsection{The impact of gender and age}

As presented in the previous section, our results revealed no significant differences among subjects for the success variable with respect to gender or age and that completion time is not affected by gender but is affected by age. The lack of gender 
Kindertivity: Usability and Communicability Strategies for Interactive Surfaces and PreKindergarten Children

differences is perhaps surprising, given that previous work shows superior fine motor control in girls and that male toddlers' hands tend to be larger. In this respect, we have to point out that the average completion times for girls are in general lower than for boys for gestures that require precision, such as scale up, scale down and one finger rotation. However, the differences are not big enough to obtain statistical significance. It is also interesting to observe that the Age*Gender interaction has a nearly statistical significant effect ( $\mathrm{p}$-value $=0.094$ ) on the degree of success variable (see Table 5) for the scale up and scale down tasks, but further research would be needed to verify whether hypothesis $\mathrm{H}_{34}$ (see Table 4) can be rejected for these tasks.

The results regarding the age factor are in accordance with the fact that children start to develop their preoperational stage at 24 months and gradually acquire fine-grained motor skills after this time. This is notably the case for double tap, drag, scale down and one-finger rotation tasks, in which the participants in the second age group (31 to 38 months) perform faster than those in the first (24 to 30 months). However, this agerelated enhancement process was not observed for scale up and two-finger rotation interactions, due to the interference, precision and cognitive complexity issues described in Section 5.5.4 affecting both age ranges. These issues will open up a new interaction design strategy for children in which gestures with different levels of difficulty could be automatically set according to factors such as age and, eventually, gender. Thresholds, rotation angles and directions, etc. could be set automatically to make interfaces more or less demanding for children, according to their different characteristics and the nature of the learning activity to be carried out.

\subsubsection{Designing multi-touch applications for pre-kindergarteners}

The above findings, based on the quantitative results, are not the only factors that should be taken into consideration when implementing interactive applications for prekindergarteners. There are also lessons to be learned from the qualitative observations in this study that may have implications for the way multi-touch interactions should be designed.

In the first place, the fact that children are able to perform most of the considered gestures but experience difficulties with certain movements that need more highly honed skills, suggests that some interaction aids need to be provided. Special attention must be paid to the finalization phase of gestures with high levels of precision (see Figure 32 right). In our opinion, this is not always feasible at this early age and the implementation of boundary detection components that detect when an object gets close to its final desired state should always be considered. In this respect, our first design guideline (DG) can thus be stated as follows:

(DG1) Boundary Final/Exit Conditions: Design boundary detection components to cope with precision problems related to the exact final state of an interactive element. 
Another important issue that needs careful consideration is the initial phase of some touch interactions, as in the case of the long press gesture. A possible solution to avoid the initial dragging events is to consider a filtering process that would be activated when this gesture is required. In general, designers should take these issues into consideration by following our second design guideline:

(DG2) Spurious Initial/Entry Events: Design filtering processes for spurious undesired events that may occur in the acquisition/initial phase of any interaction.

There are also motor limitations that may affect the speed at which a given interaction may be performed. In general, any interaction with associated time restrictions should take into account the lowest speed at which it may effectively be performed by prekindergarteners:

(DG3) Time-based interactions: Design adaptive mechanisms to match the required speed for time-based interactions to the actual ability of the user.

Finally, cognitive complexity has to be adequately managed when designing touch interactions. We have observed performance issues with gestures requiring more advanced skills that require mathematical thinking, bi-manual coordination and spatialgeometric interpretation to plan a complex gesture in advance. Some previous results in the literature have shown that this is also important for adults (Nacenta, Baudisch, Benko, \& Wilson, 2009), although not all manipulations might require this planning in advance, which makes it an even more important point for designers. These aspects should be addressed by considering our three final design guidelines:

(DG4) Mathematical thinking interactions: Avoid including interactions that involve mathematical concepts such as counting exact numbers or repeating a given number of events.

(DG5) Coordinated interactions: Avoid interactions that require complex coordination processes involving both hands.

(DG6) Geometric-based interactions: Avoid interactions that require the effective estimation of spatial relationships if performance is a mandatory requirement.

We believe the above recommendations will open up an interesting area of research on the design of adaptable interfaces for pre-kindergarteners that match their interaction requirements and challenges to their actual abilities. Additionally, it would also be of interest to design semiotic systems that include audio-visual feedback to improve the communicability of these interaction problems. These systems would motivate children to improve their interactions.

\subsubsection{Revisiting multi-touch interactions for adults}


Kindertivity: Usability and Communicability Strategies for Interactive Surfaces and PreKindergarten Children

Besides the quantitative and qualitative findings discussed above, there are specific issues related to previous studies with adults that strengthen the idea that existing multi-touch interaction models must be tailored to cope with the specific characteristics of pre-kindergarten children.

Firstly, our results obtained from the pinch gesture with pre-kindergarteners disagree with those reported for adults in (Hoggan, Nacenta, et al., 2013). Adult users in general perform contracting pinch gestures faster because this gesture is ergonomically easier for them. As pointed out by the authors of this study, the average rotation amplitude of the index finger inter-phalangeal joint is lower for contraction than expansion. In the absence of the cognitive development problem, the main issue when considering pinch gestures by adults is ergonomic. It is interesting to note that longer distances cause significantly more ergonomic failures with adults. Consequently, as suggested in this study, it would also be worth investigating whether non-direct mappings between finger distance and zoom or scaling magnitude might improve pre-kindergartners' efficiency in this respect.

Secondly, we should not be surprised that rotations are the gestures requiring the highest level of cognitive effort in this study, as they involve some of the most complex motor skills among common multi-touch gestures. In this respect, recent studies with adult subjects (Hoggan, Williamson, et al., 2013) show surprising interaction effects among the variables studied (angle, diameter, direction and position) in rotations performed with the index finger and thumb. It remains to be seen whether there are also categories of rotations (both two and one finger-based) at the earliest stages of motor development that may cause problems, as occurs with adult subjects. The study of anti-clockwise rotations and the presence of large diameters and angles should provide useful information on heuristic aids in design.

Finally, if we compare the execution times of the drag, one-finger rotation, scale up and scale down gestures against the regression model of multi-touch manipulation proposed for adults by (Zhao, Soukoref, \& Balakrishnan, 2011), we can conclude that our observed execution times do not fit this model. Namely, children under 30 months perform these gestures on average 8.5 times slower than adults, whereas children over 30 months perform them 4.1 times slower. The differences between boys and girls are not so wide, with girls performing these gestures on average 5.6 times slower than adults and boys are 6.8 times slower. These results reveal that additional research is needed to obtain a specific application of the Mahalanobis distance metric to the index of difficulty equation from Fitts' law, in order to properly model multi-touch manipulations by children in this age range. However, taking into account the current state of the art, it is not clear whether this type of modelling is feasible for children, as the number of dimensions that affect multi-touch interactions in this early age range may be high, making it difficult to obtain a model, as Zhao et al. (Zhao et al., 2011) recognized. 


\subsubsection{Applications beyond $\mathrm{HCI}$}

Finally, the results obtained are not only of interest to interaction designers but also to researchers who investigate the physical and cognitive load of these gestures in general. Studying children of different ages may provide an indication of which gestures do require a certain level of cognitive development. Additionally, the increasing use of gesture-based interfaces in the very early stages of cognitive development may also be of interest to those who investigate the role of gestures in talking and thinking. In this respect, it has been observed that children exploit hand movements at the very earliest stages of language learning. However, as pointed out by Damon et al. (Damon et al., 2006), they gradually reduce symbolic gestures as they develop. It remains to be seen whether the increased use of gesture-based interfaces by children, such as those proposed in this study, has any impact on the way they use gestures for non-verbal communication.

In addition, increasing the number of gestures in educational applications may have an effect on the design of instructional strategies for pre-kindergarteners. For example, applications that force children to perform gestures in a given sequence or according to any other pre-established requirements may contribute to the internalization of rules. This is related to behavior control, which is one the foundational skills that must be acquired at this early age. Other abilities that could be developed with the inclusion of these additional gestures are the control of attention, creativity, classification, patterning and motor planning skills.

\subsection{Threats to Validity}

There are some threats in terms of the generalization of the findings of this study to other contexts and environments and several precautions must be taken.

The fact that children were able to successfully complete certain gestures after training with an adult does not mean that they will be able to perform these without guidance or by themselves. Certainly, this issue needs further research and appropriate automated guidance systems should be designed to overcome this problem.

With respect to time-based interactions, another threat is that the results are very likely dependent on the specific thresholds chosen, namely, on double taps and long pressed interactions. It is possible that relaxing these thresholds would make these gestures much more successful.

Two limitations must be considered with regard to rotation gestures. Firstly, success was achieved when the target angle was surpassed and not when the object reached a specific orientation within certain error limits. Additional experiments would be needed to verify whether this additional precision requirement would have a significant impact on performance. Secondly, the designed two-finger rotation in the end turned out to be a rather complicated way of rotating, which the children were not able to 
Kindertivity: Usability and Communicability Strategies for Interactive Surfaces and PreKindergarten Children

understand. This severely affected the performance of this gesture, so that a less confusing bi-manual rotation procedure should be designed and evaluated.

In relation to scaling gestures, it is also important to note that scale up and down were not separated into one-hand or multiple-hand gestures and this could have introduced noise into the measure.

Additionally, although gestures in the experimental setting were evaluated in isolation, in certain contexts they happen consecutively and therefore some of the results might not apply in these cases. In fact, although we tend to think that gestures are isolated and instantaneous, there is some evidence (Hinrichs \& Carpendale, 2011) that some of them are affected by previous and subsequent events. In this respect, the results of the study are necessarily reductionist and they have not revealed the limits to combining the different gestures, i.e., we have not considered situations in which a designer might need multiple gestures to be carried out at the same time.

Another limitation of our work is related to applications that generally require touch gestures to be complete and separated from each other by "release". Although applications could be designed that work on thresholds, explicit gesture separation events (release) could be needed, and these have not really been studied in this experiment.

Finally, we still need to study the effect of space cluttering in situations in which several interactive elements need to be displayed simultaneously, leaving users with a restricted interaction area. It is not known whether a limited space would make these gestures less successful for pre-kindergarten children.

\subsection{Conclusions and future work}

In this work we analyzed a corpus of 100 commercial applications running on multitouch devices for pre-kindergarteners and concluded that $99 \%$ of the applications used tap and 56\% used drag gestures as their only supported operations. In order to analyze very young children's capacity to successfully perform additional gestures we designed an experimental evaluation in which 2-3 year old children participated as users of a multi-touch application requiring diverse types of operations, including tap, double tap, long pressed, drag, scale up, scale down, and one and two finger rotation.

Our findings provide evidence that additional gestures (one-finger rotation and twofinger scale up and scale down) may be effectively incorporated into applications targeting pre-kindergarten users and running on multi-touch devices with comparatively little implementation effort. Other gestures (double tap and long pressed) may also be considered, provided precision and cognitive limitations are taken into account. The analysis of these limitations gave us a set of design guidelines that address boundary exit conditions, spurious entry events, time, counting, distance-based and coordinated interactions. 
Despite the conclusions obtained, the work presented in this paper is the first step in a study of multi-touch gestures with very young children and opens a new area of research with many pending questions and interesting issues to be addressed in future work.

There are some gestures, such as double tap and long pressed, that could be implemented differently to improve the success rate by taking into account the observed issues. For instance, the time gap for the double tap could reasonably be prolonged or dynamically adapted to children's different skill levels and the short spurious movements detected when trying to keep the finger still in the long pressed gesture could be filtered out. An improved technique for the drag gesture could also be considered, such as the one used in (Rick et al., 2010) and (Harris et al., 2009), which filters out temporary skipping of the finger. Although these solutions seem feasible, all these techniques will require further empirical evaluation and validation, both in isolation and when put together in a single application.

Obtaining detailed information on the accuracy with which gestures can be performed is also an interesting strand of future work. Good examples include how accurately they can rotate an object or how close they can drag an object to a target. This would certainly help in understanding the limitations and how demanding applications should be as regards the precision of a given gesture.

Another interesting issue is that of unexpected touching when holding the tablet with a finger resting on the display or when part of the palm also touches the surface if it is not carefully approached (Mansor et al., 2009). This is a difficult issue to address because children may not realize that such unintentional contacts with other parts of their body when their fingers approach the screen have a different effect to what happens when they use paper and tangible materials. It would therefore be interesting to explore potential improvements in multi-touch usability, for instance by determining and filtering out unexpected blob contacts wherever applicable. In this respect, works such as those of (Schwarz, Xiao, Mankoff, Hudson, \& Harrison, 2014) and (Vogel \& Casiez, 2012), who studied the detection of different types of contacts and occlusion patterns on multi-touch surfaces, could be used as a starting point.

Further research will be needed to design effective two-finger rotations for this age range. Attentional and motivational factors are also important in moderating motor capabilities that lead to performing gestures successfully. Thus, we also plan to investigate the suitability of existing semiotic approaches, such as those proposed by Derboven et al. (Derboven, De Roeck, \& Verstraete, 2012), to advise users of the gestures they are expected to perform in multi-touch applications for prekindergarteners. We must also be aware of how fast many children are becoming familiar with multi-touch devices by gaining access to their parents' tablets. This exposure to multi-touch technology should have a positive effect on the way they learn and acquire abilities to perform gestures. We must therefore be on the lookout for any design guidelines and gestures that could change as soon as this situation is prolonged 
Kindertivity: Usability and Communicability Strategies for Interactive Surfaces and PreKindergarten Children

over time, and which ones will still apply, as motor or cognitive skills are not significantly altered despite this higher exposure and experience.

All the previous issues are worth studying in an extended age range, also involving children in the $4-5$ age range. It would be particularly interesting to observe whether this extended study with an increased number of subjects exposes gender differences that were not observed in the present study.

Finally, we wonder whether users with special needs or motor restrictions could take advantage of gestures personalized to their motor skills. Although such personalization must be performed on a case-by-case basis, future work in this respect could be focused on exploring how basic gestures under typical motor restrictions can be adapted to improve usability and performance when using touch-enabled displays.

\section{Acknowledgments}

We would like to thank the Universitat Politécnica de Valéncia ${ }^{3}$ and the ItuItu ${ }^{4}$ Prekindergarten Schools for their collaboration in this study as well as the anonymous reviewers for their helpful comments. This research has been funded by the Spanish Ministry of Economy and Competitiveness and by the FEDER funds of the EU under the project Grants CreateWorlds (TIN2010-20488) and insPIre (TIN2012-34003). The work is also supported by a postdoctoral fellowship within the VALi+d program from Conselleria d'Educació, Cultura i Esport (Generalitat Valenciana) to A. Catalá (APOSTD/2013/013). Vicente Nácher received a Research Collaboration Scholarship (Project 2012/32/00004) for this work from the Spanish Ministry of Education.

3 ampa.blogs.upv.es

4 www.ituitu.es 


\section{Appendix A}

Table 10. Time in milliseconds to perform the tasks by age

\begin{tabular}{|c|c|c|c|}
\hline Task & Age Group & Average & SD \\
\hline \multirow{3}{*}{ Tap } & $<=30$ & 5140.94 & 3912.07 \\
\hline & $>30$ & 3135.30 & 1957.64 \\
\hline & Total & 4138.12 & 3209.01 \\
\hline \multirow{3}{*}{ Double Tap } & $<=30$ & 5815.72 & 3326.45 \\
\hline & $>30$ & 3144.81 & 1201.32 \\
\hline & Total & 4480.26 & 2800.59 \\
\hline \multirow{3}{*}{ Long Pressed } & $<=30$ & 10652.62 & 7294.92 \\
\hline & $>30$ & 8075.00 & 3979.02 \\
\hline & Total & 9307.78 & 5817.10 \\
\hline \multirow{3}{*}{ Drag } & $<=30$ & 16017.06 & 11485.80 \\
\hline & $>30$ & 7620.94 & 3711.30 \\
\hline & Total & 11819.00 & 9417.58 \\
\hline \multirow{3}{*}{ Scale Up } & $<=30$ & 8072.42 & 6340.66 \\
\hline & $>30$ & 6065.68 & 5191.55 \\
\hline & Total & 7002.16 & 5744.57 \\
\hline \multirow{3}{*}{ Scale Down } & $<=30$ & \begin{tabular}{|l|}
7802.85 \\
\end{tabular} & 6044.72 \\
\hline & $>30$ & 2372.95 & 1221.70 \\
\hline & Total & 4906.91 & 4974.20 \\
\hline \multirow{3}{*}{ One-Finger Rotation } & $<=30$ & 11108.70 & 6618.13 \\
\hline & $>30$ & 6240.68 & 3611.40 \\
\hline & Total & 8596.17 & 5751.21 \\
\hline \multirow{3}{*}{ Two-Finger Rotation } & $<=30$ & 14920.21 & 9253.39 \\
\hline & $>30$ & 10519.61 & 4509.51 \\
\hline & Total & 12279.85 & 6943.47 \\
\hline
\end{tabular}


Kindertivity: Usability and Communicability Strategies for Interactive Surfaces and PreKindergarten Children

Table 11. Time in milliseconds to perform the tasks by gender

\begin{tabular}{l|l|l|l}
\hline \multirow{3}{*}{ Task } & Gender & Average & SD \\
\hline \multirow{3}{*}{ Double Tap } & $\mathrm{F}$ & 4656.73 & 4004.34 \\
\cline { 2 - 4 } & $\mathrm{M}$ & 3619.51 & 2161.85 \\
\cline { 2 - 4 } & Total & 4138.12 & 3209.01 \\
\hline \multirow{3}{*}{ Long Pressed } & $\mathrm{F}$ & 3728.94 & 2403.49 \\
\cline { 2 - 4 } & $\mathrm{M}$ & 5231.58 & 3064.52 \\
\cline { 2 - 4 } & Total & 4480.26 & 2800.59 \\
\hline \multirow{3}{*}{ Drag } & $\mathrm{F}$ & 9117.32 & 7356.77 \\
\cline { 2 - 4 } & $\mathrm{M}$ & 9515.55 & 3849.63 \\
\cline { 2 - 4 } & Total & 9307.78 & 5817.10 \\
\hline \multirow{3}{*}{ Scale Up } & $\mathrm{F}$ & 13976.86 & 11160.55 \\
\cline { 2 - 4 } & $\mathrm{M}$ & 9661.14 & 6985.93 \\
\cline { 2 - 4 } & Total & 11819.00 & 9417.58 \\
\hline \multirow{3}{*}{ Scale Down } & $\mathrm{F}$ & 5655.44 & 4380.83 \\
\cline { 2 - 4 } & $\mathrm{M}$ & 8541.29 & 6832.87 \\
\cline { 2 - 4 } & Total & 7002.16 & 5744.57 \\
\hline \multirow{3}{*}{ One-Finger Rotation } & $\mathrm{F}$ & 4150.58 & 4017.93 \\
\cline { 2 - 4 } & $\mathrm{M}$ & 5771.29 & 5921.07 \\
\cline { 2 - 4 } & Total & 4906.91 & 4974.20 \\
\hline \multirow{2}{*}{ Two-Finger Rotation } & $\mathrm{F}$ & 7653.27 & 5163.65 \\
\cline { 2 - 4 } & $\mathrm{M}$ & 9480.15 & 6288.45 \\
\cline { 2 - 4 } & Total & 8596.17 & 6751.21 \\
\hline & $\mathrm{F}$ & 12760.13 & 7508.27 \\
\cline { 2 - 4 } & $\mathrm{M}$ & 11799.57 & 6700.27 \\
\cline { 2 - 4 } & Total & 12279.85 & \\
\hline
\end{tabular}


Multi-touch gestures for pre-kindergarten children

Table 12. Success rate in each task by age groups

\begin{tabular}{|c|c|c|c|}
\hline Task & Age Group & Average & SD \\
\hline \multirow{3}{*}{ Tap } & $<=30$ & \begin{tabular}{|l|}
0.9375 \\
\end{tabular} & 0.25000 \\
\hline & $>30$ & 0.9375 & 0.25000 \\
\hline & Total & 0.9375 & 0.24593 \\
\hline \multirow{3}{*}{ Double tap } & $<=30$ & 0.6875 & 0.47871 \\
\hline & $>30$ & 0.5625 & 0.51235 \\
\hline & Total & 0.6250 & 0.49187 \\
\hline \multirow{3}{*}{ Long pressed } & $<=30$ & 0.5000 & 0.51640 \\
\hline & $>30$ & 0.5000 & 0.51640 \\
\hline & Total & 0.5000 & 0.50800 \\
\hline \multirow{3}{*}{ Drag } & $<=30$ & 0.9375 & 0.25000 \\
\hline & $>30$ & 0.8750 & 0.34157 \\
\hline & Total & 0.9062 & 0.29614 \\
\hline \multirow{3}{*}{ Scale up } & $<=30$ & 0.8750 & 0.34157 \\
\hline & $>30$ & 1.0000 & 0.00000 \\
\hline & Total & 0.9375 & 0.24593 \\
\hline \multirow{3}{*}{ Scale down } & $<=30$ & 0.8750 & 0.34157 \\
\hline & $>30$ & 1.0000 & 0.00000 \\
\hline & Total & 0.9375 & 0.24593 \\
\hline \multirow{3}{*}{ One-Finger Rotation } & $<=30$ & 0.8750 & 0.34157 \\
\hline & $>30$ & 0.8750 & 0.34157 \\
\hline & Total & 0.8750 & 0.33601 \\
\hline \multirow{3}{*}{ Two-Finger Rotation } & $<=30$ & 0.3125 & 0.47871 \\
\hline & $>30$ & 0.5000 & 0.51640 \\
\hline & \begin{tabular}{|l|} 
Total \\
\end{tabular} & 0.4063 & 0.49899 \\
\hline
\end{tabular}


Kindertivity: Usability and Communicability Strategies for Interactive Surfaces and PreKindergarten Children

Table 13. Success rate in each task by gender

\begin{tabular}{|c|c|c|c|}
\hline Task & Gender & Average & SD \\
\hline \multirow{3}{*}{ Tap } & $\mathrm{F}$ & 0.9375 & 0.25000 \\
\hline & $\mathrm{M}$ & 0.9375 & 0.25000 \\
\hline & Total & 0.9375 & 0.24593 \\
\hline \multirow{3}{*}{ Double tap } & $\mathrm{F}$ & 0.5625 & 0.51235 \\
\hline & $\mathrm{M}$ & 0.6875 & 0.47871 \\
\hline & Total & 0.6250 & 0.49187 \\
\hline \multirow{3}{*}{ Long Pressed } & $\mathrm{F}$ & 0.5000 & 0.51640 \\
\hline & $\mathrm{M}$ & 0.5000 & 0.51640 \\
\hline & Total & 0.5000 & 0.50800 \\
\hline \multirow{3}{*}{ Drag } & $\mathrm{F}$ & 0.9375 & 0.25000 \\
\hline & $\mathrm{M}$ & 0.8750 & 0.34157 \\
\hline & Total & 0.9062 & 0.29614 \\
\hline \multirow{3}{*}{ Scale up } & $\mathrm{F}$ & 1.0000 & 0.00000 \\
\hline & $M$ & 0.8750 & 0.34157 \\
\hline & Total & 0.9375 & 0.24593 \\
\hline \multirow{3}{*}{ Scale down } & $\mathrm{F}$ & 1.0000 & 0.00000 \\
\hline & $\mathrm{M}$ & 0.8750 & 0.34157 \\
\hline & Total & 0.9375 & 0.24593 \\
\hline \multirow{3}{*}{ One-Finger rotation } & $\mathrm{F}$ & 0.9375 & 0.25000 \\
\hline & $\mathrm{M}$ & 0.8125 & 0.40311 \\
\hline & Total & 0.8750 & 0.33601 \\
\hline \multirow{3}{*}{ Two-Finger rotation } & $\mathrm{F}$ & 0.4375 & 0.51235 \\
\hline & $\mathrm{M}$ & 0.3750 & 0.50000 \\
\hline & Total & 0.4063 & \begin{tabular}{|l}
0.49899 \\
\end{tabular} \\
\hline
\end{tabular}




\title{
Chapter 6
}

\section{Improving \\ Pre-Kindergarten \\ Touch Performance}

Vicente Nacher ${ }^{1}$, Javier Jaen ${ }^{1}$, Alejandro Catala ${ }^{1}$, Elena Navarro ${ }^{2}$, Pascual Gonzalez ${ }^{2}$

${ }^{1}$ ISSI Group, Dpto. Sistemas Informáticos y Computación, Universitat Politècnica de València.

${ }^{2}$ LoUISE Research Group, Computing Systems Department, University of Castilla-La Mancha.

Published in Proceedings of the 9th ACM International Conference on Interactive Tabletops and Surfaces(ITS'14), pp. 163-166. ACM (2014)

\begin{abstract}
Multi-touch technology provides users with a more intuitive way of interaction. However, pre-kindergarten children, a growing group of potential users, have problems with some basic gestures according to previous studies. This is particularly the case of the double tap and long pressed gestures, which have some issues related to spurious entry events and time-constrained interactions, respectively. In this paper, we empirically test specific strategies to deal with these issues by evaluating off-the-shelf implementations of these gestures against alternative implementations that follow these
\end{abstract}


Kindertivity: Usability and Communicability Strategies for Interactive Surfaces and PreKindergarten Children

guidelines. The study shows that the implementation of these design guidelines has a positive effect on success rates of these two gestures, being feasible their inclusion in future multi-touch applications targeted at pre-kindergarten children.

\subsection{Introduction}

Multi-touch technology provides users with a more intuitive way of interaction (Smith et al., 2012) and, nowadays, it is widespread accepted. This fact has boosted a new approach to developing applications for children. As pointed out in (Rideout, 2011), children between zero and eight years old are frequent users of digital media in the USA.

Recent work in (Nacher et al., 2015) presented an empirical evaluation of a set of multi-touch gestures and their suitability for children between two and three years. This work pointed out that some basic interactions (e.g. tap, drag, scale up, scale down and one finger rotation) can be performed effectively and could, therefore, be included in educational applications for pre-kindergarten children. However, it also revealed some issues with the long pressed and double tap gestures because fine motor skills are still under development at this early age and because of the cognitive complexity of some interactions. Firstly, in the long pressed gesture, the spurious initial or entry dragging events produced by the lack of precision of pre-kindergarteners prevent the system from detecting the gesture. Secondly, in the double tap gesture the standard time required between taps is much shorter than the actual pace at which pre-kindergarteners are able to perform. Authors of this previous work stated a set of design guidelines to deal with, among others, these specific issues. Regarding the spurious entry events in the case of the long pressed gesture, we consider in this work a filtering process to ignore these initial dragging events. On the other hand, we can adjust the required speed for the double tap interaction to the actual ability of the target user. This paper addresses the evaluation of these two assisted strategies for pre-kindergarteners to validate empirically their effectiveness.

\subsection{Related work}

There are some interesting studies targeted at children that focus on the direct manipulation interaction style and direct touch. For instance, the works in (Couse \& Chen, 2010) and (Terra et al., 2009) involved subjects aged between 3 and 6, and between 9 and 11, respectively, in testing the use of the stylus for learning purposes. They concluded that the stylus interaction had a shorter learning period and other advantages over the traditional mouse and keyboard alternatives.

The differences between single-touch and multi-touch interaction were analyzed in (Harris et al., 2009) for 7 to 10 year-old children's groups. Results pointed out that although touch did not have an effect in the interaction in terms of frequency or equity, 
it did influence the communication of subjects and encouraged them to talk to each other about their collaborative actions.

There are several studies that have addressed the different issues affecting multi-touch interaction with adult users(Hoggan, Nacenta, et al., 2013; Hoggan, Williamson, et al., 2013; Nacenta et al., 2009). However, these users under normal conditions are not affected by entry precision problems or cognitive-motor issues as pre-kindergarteners are.

\subsection{Experimental study}

The overall goal of our experimental study is to identify whether the proposed assisted strategies, filtering initial spurious dragging events and adjusting the required speed between taps, for the long pressed and double tap gestures actually contribute to overcome the problems identified in (Nacher et al., 2015). More specifically, the present study research questions are formulated as follows:

- Do the implemented assisted strategies for the gestures under study (i.e. long pressed and double tap) have a positive effect on pre-kindergarteners' performance?

- In the specific case of the gestures following the design guidelines, is there a significant relationship between gesture performance and gender or age group?

In order to answer these questions we need to contrast the implemented gestures following the design guidelines against their baseline implementations without guidelines, i.e., applied as provided by current OS runtimes (e.g. Android). To this purpose, we took the existing dataset from (Nacher et al., 2015), which already evaluated both the success rate and the completion time for the baseline implementation, and then we carried out an experiment that follows the same procedure to collect the data observations with the implementation of the assisted gestures.

\subsubsection{Participants}

Thirty-two children aged between twenty-two and forty-six months took part in the experiment of the two gestures with design guidelines $($ Mean $(M)=31.31$, Standard Deviation $(\mathrm{SD})=6.25)$. The genders of the children were balanced. Parental authorization was obtained before carrying out the study. The children were divided, as in the previous study (Nacher et al., 2015), into two age groups: 22 to 30 months and 31 to 46 months, with 8 males and 8 females per group.

\subsubsection{Apparatus}

The interaction framework for the experiment was implemented in Java using JMonkeyEngine SDK v.3.0beta. The devices used for the experiment were a Motorola MZ601 and a Samsung Galaxy Note 10.1 tablet with Android 3.2 both with capacitive multi-touch screens. 
Kindertivity: Usability and Communicability Strategies for Interactive Surfaces and PreKindergarten Children

\subsubsection{Tasks}

\subsubsection{Long Pressed}

A static image of an animal appears in a random position on the screen (see Figure 33). Participants are requested to carry out a long pressed gesture on the target image until the target disappears. The task will succeed when the participants put their finger in the target image and hold it at least 500 milliseconds. The implemented design guideline consists of a filtering process for ignoring the spurious undesired interaction events that may occur at the beginning of the gesture. The filtering ignores any short drag gesture (less than $1 \mathrm{~cm}$ ) around the first initial contact point.

\subsubsection{Double Tap}

A static image of an animal appears in a random position on the screen (see Figure 33). Participants are requested to double tap on the target image with one finger in order to pass the test.

The default allowed interval for the double tap gesture in Android devices is 300 milliseconds. Therefore, we have considered as a design guideline higher time intervals up to 2000 milliseconds to study their impact on the success rate for this gesture.

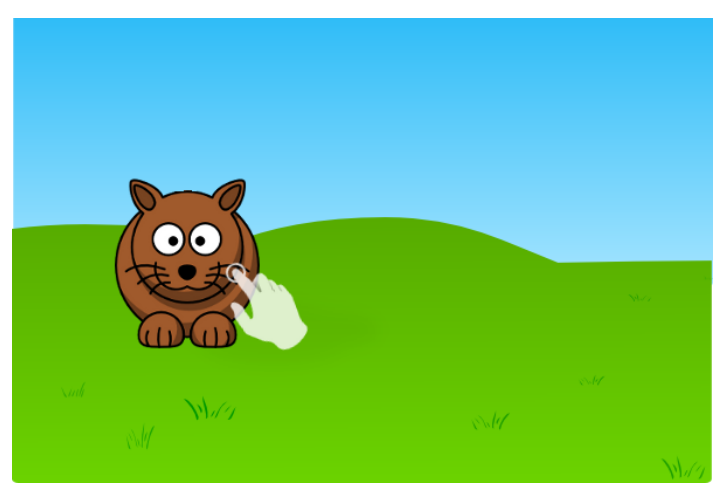

Figure 33. Example of long pressed and double tap task.

\subsubsection{Procedure}

For each task, the children participated in a 5-minute learning session with an instructor. Then, the experimental platform asked them to perform the task with no external adult intervention. They had to perform three repetitions of each gesture as described in the Tasks section. When the gesture was successfully completed, the platform gave a positive audiovisual feedback. If the instructor observed that the participant did not carry out the task in a given time, it was marked as undone and the child went on to the next one. 
For each interaction, the system recorded the start time (seconds needed to go into action after the visual stimulus was shown), completion time (milliseconds until the gesture was completed), success (performed correctly or incorrectly), and in the case of the double tap gesture, the time elapsed between taps. Additional notes were taken by an external observer during the experimental sessions to register any incidental information that could be worth discussing later.

\section{4. $\quad$ Results}

\subsubsection{Assisted vs non-Assisted Interaction}

In the following, we will refer to the technique in which the design guidelines were implemented as "assisted" interaction. We will contrast these data against the dataset provided by the authors of (Nacher et al., 2015), to which we will refer as "nonassisted" interaction.

\subsubsection{Success Rate}

In order to aggregate the success variable over the three repetitions, if a participant performed successfully either zero or one tests in a specific task, they were considered incapable of performing it. On the other hand, if they successfully performed two or three tests in a specific task, they were considered capable of doing it because they actually showed their ability to consistently reproduce the gesture several times.

Table 14. Success rate by task and technique

\begin{tabular}{|c|c|c|c|}
\hline Task & Technique & Mean & SD \\
\hline Long Pressed & non-Assisted & 0.5000 & 0.5080 \\
\cline { 2 - 4 } & Assisted & 0.9062 & 0.2961 \\
\hline \multirow{2}{*}{$\begin{array}{c}\text { Double Tap } \\
\text { (1200 ms) }\end{array}$} & non-Assisted & 0.6250 & 0.4919 \\
\cline { 2 - 4 } & Assisted & 0.8438 & 0.3689 \\
\hline
\end{tabular}

According to this codification, the success rate in the long pressed task can be expressed as a percentage, as shown in Table 14 for each task and technique. Additionally, to enable a fine-grained analysis of this variable when considering the double tap gesture, we have studied how the success rate would be for different maximum allowed times between consecutive taps as shown in Figure 34.

As hypothetized, the implementation of assisted techniques following the suggested guidelines increases the success rate for both gestures. A Pearson's chi-squeare test showed that there is a significant strong relationship between the carried out technique and the success rate for the long pressed task (Pearson $\chi^{2}(1, N=64)=12.650$, $\mathrm{p}<0.001)$. Regarding the double tap task, the minimum time interval between taps for which we found significant differences in the variable success rate between techniques was 1200 milliseconds $\left(\chi^{2}(1, N=64)=3.925, \mathrm{p}=.048\right)$. If lower intervals are considered, the success rates obtained are not significantly different from the ones 
Kindertivity: Usability and Communicability Strategies for Interactive Surfaces and PreKindergarten Children

obtained with the Android standard 300ms interval. Higher intervals do not provide significantly better success rates.

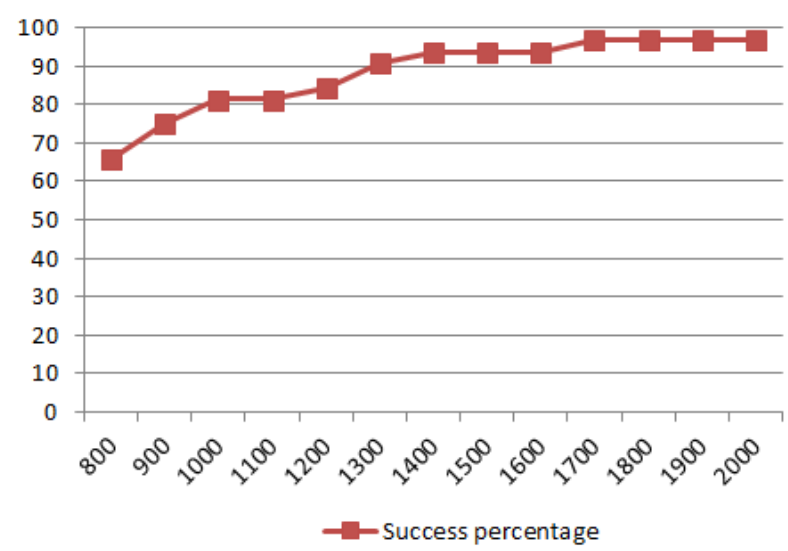

Figure 34. Success rate by maximum allowed time between taps in milliseconds

\subsubsection{Completion Time}

The average of each subject's successful tasks is used to obtain the average completion time (see Table 15) value per task and technique. If the test is not performed successfully it is not included in the completion time analysis.

Table 15. Completion time (msec.) by task and technique

\begin{tabular}{|c|c|c|c|}
\hline Task & Technique & Mean (s.) & SD \\
\hline \multirow{2}{*}{ Long Pressed } & non-Assisted & 9307.78 & 5817.10 \\
\cline { 2 - 4 } & Assisted & 8053.77 & 6070.38 \\
\hline \multirow{2}{*}{ Double Tap } & non-Assisted & 4480.38 & 2800.65 \\
\cline { 2 - 4 } & Assisted & 3332.16 & 2292.82 \\
\hline
\end{tabular}

The assisted techniques took less time to complete with respect to the baseline implementation, although differences were not found statisically significant. In this regard one-way between-subject ANOVAs with the independent variable technique and the dependent variable completion time demonstrated that there is not a significant main effect of the technique for neither the long pressed $(\mathrm{F}(1,54)=.584, \mathrm{p}=.448)$ nor the double tap task $(\mathrm{F}(1,52)=3.393, \mathrm{p}=.071)$.

\subsubsection{Age and Gender Influence}

As it was previously stated, we are also interested in knowing whether the implemented design guidelines have a different effect in the response variables (success rate and completion time) with respect to age or gender. This is interesting given that a prior study (Nacher et al., 2015) found that only age has an effect on completion time for the double tap with no assisted implementation, and that there are no significant differences on the success rate by age or gender. 


\subsubsection{Success Rate}

No significant differences on the sucess rate for both tasks were found by either age or gender. This is comfirmed by the Pearson's chi-square tests on the long pressed success rate by the gender factor $(\chi 2(1, \mathrm{~N}=32)=0.368, \mathrm{p}=.544)$ or by the age group factor $(\chi 2(1, \mathrm{~N}=32)=3.310, \mathrm{p}=.069)$; as well as on the double tap success rate by gender $\left(\chi^{2}(1, \mathrm{~N}=32)=0.237, \mathrm{p}=.626\right)$ and age group factor $\left(\chi^{2}(1, \mathrm{~N}=32)=2.133\right.$, $\mathrm{p}=.144)$.

\subsubsection{Completion Time}

In this respect, older children performed significantly faster only with the long pressed gesture. No other effects were found significant for any gesture and factor. Specifically, a two-way between-subjects ANOVA on the completion time for the long pressed task revealed a significantly main effect of the age group factor $(\mathrm{F}(1,31)=$ 8.751, $\mathrm{p}=.006)$. Completion time doubled for the youngest group on average $\left(\mathrm{M}_{\text {young }}=11.1 \mathrm{~s}\right.$ and $\left.\mathrm{M}_{\mathrm{old}}=5.2 \mathrm{~s}\right)$. There was no significant main effect of the gender factor $(\mathrm{F}(1,31)=0.559, \mathrm{p}=.461)$.

The corresponding analogous statistical analysis for the double tap task did not revealed significant main effects of age group $(\mathrm{F}(1,28)=0.135, \mathrm{p}=.717)$ or gender $(\mathrm{F}(1,28)=0.017, \mathrm{p}=.897)$.

\subsection{Discussion and Conclusions}

The design guideline implemented for the long pressed gesture comprises a filtering process to ignore initial dragging events that are produced as a consequence of a motor precision problem which was identified in previous experiments. The results show a statistically significant better success rate $(90.62 \%)$ when the filtering is applied than the one observed in previous experiments without filtering (50\%). The improved success rate when the filtering is applied is not affected by age and gender. Moreover, the average completion time when the filtering is applied is not significantly different from the completion time obtained without filtering. However, as it was the case when no filtering was present, younger children need a significantly higher time to complete the task than older ones. This is because younger children are less precise and perform significantly more undesired dragging events that are larger than $1 \mathrm{~cm}$ in size. These were not removed by the implemented filter, forcing users to restart the interaction. These very young users would need a filtering process coping with larger undesired dragging events. Nevertheless, this may result problematic for interactive objects that may accept both long pressed and dragging events because dragging operations would not start until the size threshold of the filter would be surpassed.

The design guideline for the double tap gesture proposes the implementation of an extended time interval between consecutive taps. The results show that, if the default time interval between taps for Android devices (300ms) is increased to $1200 \mathrm{~ms}$, we 
Kindertivity: Usability and Communicability Strategies for Interactive Surfaces and PreKindergarten Children

obtain significantly better success rates $(62 \%$ success @ 300ms versus $84.38 \%$ success @ $1200 \mathrm{~ms}$ on average). Smaller time intervals do not provide significantly better success rates when compared to the default Android time interval. The improved success rate, when the new $1200 \mathrm{~ms}$ time interval is used, is independent of age and gender. In addition, the results show that the completion time for this task has no significant main effects of age or gender. However, it is worth mentioning that we have carried out additional trials considering external stimuli to motivate children to interact quicker (i.e. encouraging them to go beyond their natural pace). These preliminary tests suggest that it is possible to obtain faster successful double tap interactions (a success rate of $91.67 \%$ with a $600 \mathrm{~ms}$ gap) in the presence of adults motivating prekindergarteners. This will be the subject of a future experimental design considering factors such as age, gender, source (human or computer) type and intensity of the stimuli. It must also be taken into account that these stimuli may have some counter effects that have already been observed such as subjects performing more than two taps in the presence of stimuli or increased stress levels when children are continuously encouraged to perform faster than their natural pace.

To sum up, the obtained results demonstrate that the inclusion of the proposed design guidelines make these two gestures more feasible for pre-kindergarten children. These two gestures (double tap and long pressed) complete now the previous set (Nacher et al., 2015) of feasible gestures (tap, drag, scale-up, scale-down and one-finger rotation) that may be effectively performed in this early age range, what contributes to the continuous enhancement of assisted techniques for this challenging target user group of very young children.

\section{Acknowledgments}

This work received financial support from the Spanish Ministry of Education under the National Strategic Program of Research and Projects TIN2010-20488 (CREATEWORLD) and TIN2012-34003 (insPIre). This work is also supported by a postdoctoral fellowship within the $\mathrm{VALi}+\mathrm{d}$ program from Conselleria d'Educació, Cultura i Esport (Generalitat Valenciana) to A. Catalá (APOSTD/2013/013). 


\title{
Chapter 7
}

\section{Evaluating the \\ Accuracy of Pre- \\ Kindergarten Children Multi-touch Interaction}

Vicente Nacher, Javier Jaen

${ }^{1}$ ISSI Group, Dpto. Sistemas Informáticos y Computación, Universitat Politècnica de València.

Published in 15th IFIP TC.13 International Conference on Human-Computer Interaction (INTERACT'15), pp.549-556. Springer (2015)

\begin{abstract}
The direct manipulation interaction style of multi-touch technology makes it ideal for pre-kindergarten children. Recent studies have shown that these challenging users are able to perform a set of basic multi-touch gestures. However, little is known about the accuracy that they can achieve. This paper evaluates the performance of prekindergarten children when accuracy is required in the termination phase of these
\end{abstract}


Kindertivity: Usability and Communicability Strategies for Interactive Surfaces and PreKindergarten Children

gestures and points out that a mechanism for dynamically adapting the accuracy level could help children in their motor skills development.

\subsection{Introduction}

Nowadays children between zero and eight years old are frequent users of digital media (Common Sense Media, 2013). In fact, as touch allows a more intuitive and natural way of interaction (Smith et al., 2012), they are often exposed to multi-touch technology even before they learn higher oral communication skills.

This has been confirmed by recent works such as (Nacher et al., 2015) which reveals that even children between the ages of two and four are able to perform a basic set of touch gestures and (Vatavu et al., 2015) which concludes that, overall, children aged 3 to 6 years are able to perform the tap, double tap, drag \& drop and double drag \& drop gestures. However, these works have also pointed out that very young children have precision problems in both the acquisition and termination phases of these interactions because of limitations in cognitive and motor skills.

Regarding this matter, there are no studies in the literature addressing the topic of accurate performance of multi-touch gestures by pre-kindergarten children. In this paper we explore whether children aged two to three years are able to perform a set of touch gestures when high levels of precision are required and evaluate whether factors such as age and gender have an impact on the performance of these interactions by prekindergarten users. This paper contributes to a growing body of literature in the area of children-computer interaction by providing findings from a controlled experiment with four touch gestures in which the termination phase must be performed with high-levels of precision. The findings will confirm that, at this early age-range, there are significant differences among subjects with respect to precision and, therefore, designers of future touch based applications for these specific users should devise adaptive mechanisms to cope with different levels of accuracy that allow very young children to exercise and incrementally develop fine-grained touch interaction skills.

\section{2. $\quad$ Related works}

Several studies have analyzed the use of touch devices by pre-kindergarten children. The works of Abdul Aziz et al. (Abdul Aziz et al., 2013, 2014) evaluated the tap, drag, rotate, drag and drop, pinch, spread and flick gestures with children aged 2 to 4 years. Their results showed that 4 years old children were able to perform all gestures, the 3 years old ones only had some issues with the spread task and the youngest users (2 years old) were able to perform the tap and drag gestures properly but had some issues with the more complex ones.

On the other hand, the study of Nacher et al. (Nacher et al., 2015) evaluated a basic set of multi-touch gestures with pre-kindergarten children (2 to 3 years old) and concluded 
that they are able to perform gestures such as tap, drag, scale (up \& down) and one finger rotation. Moreover, the authors of this work point out that, when some proposed assisted strategies are used, pre-kindergarten children are able to perform more problematic gestures such as double tap and long press (Nacher, Jaen, Catala, et al., 2014) with high success rates.

Vatavu et al (Vatavu et al., 2015) evaluated touch gestures (tap, double tap, drag \& drop, multiple drag \& drop) with children aged between 3 and 6 years on tablets and smartphones. Their results showed that although all children had high success rates, there was a significant performance increase with age in terms of success rate and time spent performing the gesture which is an expected behavior.

These works seem to conclude that pre-kindergarten children have the necessary skills to make use of multi-touch technology. However, they assume that children may not accurately perform the multi-touch gestures under consideration and always implement assistive techniques to deal with precision issues during the initiation and termination phases of each gesture. This results in interaction styles in which pre-kindergarteners do not have the control over the termination of the gestures despite some of them have the proper cognitive abilities to perform the gestures with higher levels of precision. As a result, existing applications designed under these assumptions do not benefit from the use of multi-touch technology to help children to develop their precision-related cognitive and motor skills. Hence, in this work we evaluate the drag, scale up, scale down and rotation gestures when accurate termination of the gestures is required. The goal is to gain additional knowledge about precision issues when pre-kindergarten users are considered in the design of future touch-based applications. The results of this work would allow the design of applications that provide assistive strategies to deal with precision issues in an adaptive way only for less skilled children and not in an exhaustive way for every child as current systems do.

\subsection{Experimental study}

The overall goal of our experimental study is to identify whether pre-kindergarten children are able to get high success rates when performing gestures with high accuracy levels. More specifically, the research questions of the study are formulated as follows:

When high accuracy touch gestures are considered...

$R Q 1:$... is the degree of success independent of age group?

RQ2: ... is the degree of success independent of gender?

RQ3: ... is the completion time independent of age group?

RQ4: ... is the completion time independent of gender?

RQ5: ... is the average error independent of age group?

RQ6: ... is the average error independent of gender? 
Kindertivity: Usability and Communicability Strategies for Interactive Surfaces and PreKindergarten Children

\subsubsection{Participants}

Forty children aged between 25 and 38 months took part in the experiment (Mean (M) $=31.60$, Standard Deviation $(\mathrm{SD})=4.32$ ). Children were balanced in gender and in age group, i.e., two age groups 24 to 30 months and 31 to 38 months, with 10 males and 10 females per group were configured. Participants from two Spanish nursery schools were involved in order to explore whether children could perform non static touch gestures, i.e. requiring the movement of contacts across the surface. Parental consent was obtained before carrying out the study.

\subsubsection{Apparatus}

The interaction framework for the experiment was implemented in Java using JMonkeyEngine SDK v.3.0beta. The devices used for the experiment were a Motorola MZ601 and a Samsung Galaxy Note 10.1 tablet with Android 3.2 both with capacitive multi-touch screens.

\subsubsection{Tasks}

\subsubsection{Drag.}

A static image of an animal appears in a random position on the screen and the same (reference) image appears in a white profile in another random position, always at a distance of 588 pixels so as to be able to compare execution times among the different subjects (Figure 35-a). The random position of the reference image is subject to some geometric restrictions, to make sure that it is completely visible on the surface. Participants are requested to drag the target to the reference image with one finger. The task is successful when the target image reaches the location of the reference image with a precision of less than 15 pixels on both $\mathrm{X}$ and $\mathrm{Y}$ axis when the subject lifts his/her hand (like a drag and drop gesture).

\subsubsection{Rotation.}

A static image of an animal appears in the center of the screen in front of a blank profile of the same image in a different orientation. Rotation is always clockwise to a fixed position so as to be able to compare interaction execution times among subjects (see Figure 35-b). Participants are requested to rotate the target image to the position of the reference image by dragging one finger around the center of the target image. Pressure can be applied on the target image itself or anywhere around it. The task is successful when the target image reaches the orientation of the reference image with a precision of less than 5 degrees when the subject lifts his/her hands.

\subsubsection{Scale up.}

A static image of an animal with a size of 5 centimeters appears in the center of the screen within a similar but 1.5 times larger reference shape (see Figure 35-c). Participants are requested to scale up the target image to the size of the reference shape. 
This can be done by expanding the distance between two fingers of either one hand or two hands. The fingers do not have to be in contact with the reference image and the scaling factor applied is the incremental value returned by the JMonkeyEngine runtime for this gesture. If more than two contacts are made on the surface, JMonkeyEngine considers only the two most recent ones for communicating scaling events. The task is successful when the size difference between the manipulated and the reference images is less than 5\% when the subject lifts his/her hands.

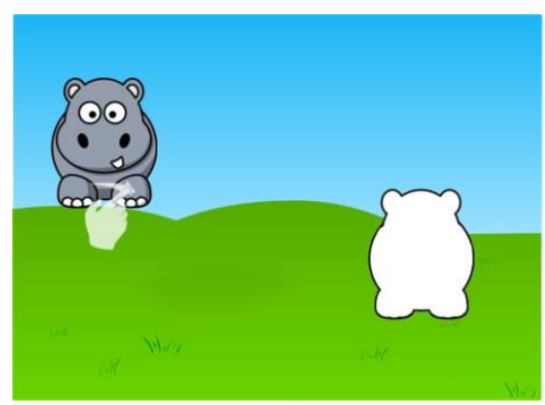

(a)

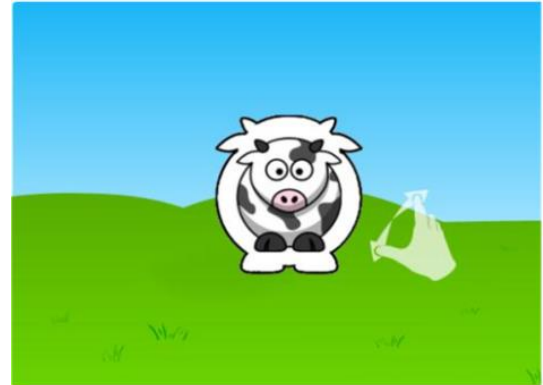

(c)

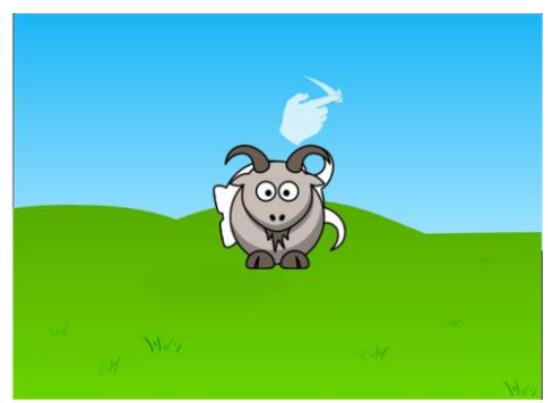

(b)

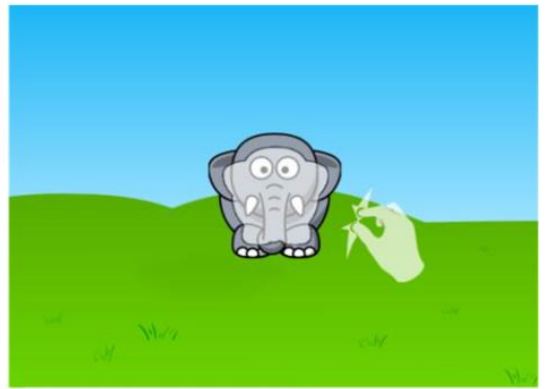

(d)

Figure 35. Example tests: (a) drag (b) rotation (c) scale up (d) scale down

\subsubsection{Scale down.}

A static image of an animal with a size of 15 centimeters appears in the center of the screen superimposed on a similar reference shape half its size (see Figure 35-d). Participants are requested to scale down the target image by making the target object shrink until it reaches the size of the reference image using two fingers of either one or two hands. The task is successful when the size difference between the manipulated and the reference images is less than 5\% when the subject lifts his/her hands.

\subsubsection{Procedure}

For each task, the children participated in a 2-minute learning session with an instructor. Then, the experimental platform asked them to perform the task with no 
Kindertivity: Usability and Communicability Strategies for Interactive Surfaces and PreKindergarten Children

external adult intervention. They had to perform five repetitions of each gesture as described in the Tasks section. When the gesture was successfully completed, the platform gave a positive audiovisual feedback. If the instructor observed that the participant did not carry out the task in a given time, it was marked as unsuccessful and the child went on to the next one. For each interaction, the system recorded the start time (seconds needed to go into action after the test began), completion time (milliseconds until the gesture was completed), success (performed correctly or incorrectly), and the number of times that users lift their hands while performing the gesture. Additional notes were taken by an external observer for posterior analysis.

\section{4. $\quad$ Results}

\subsubsection{Success}

In order to aggregate the success variable over the five repetitions, the variable was expressed as a percentage according to the number of repetitions performed successfully (Table 16).

Table 16. Success rate of each task by group

\begin{tabular}{|c|c|c|c|c|c|}
\hline \multirow{2}{*}{ Task } & \multicolumn{2}{|c|}{ Gender } & \multicolumn{2}{c|}{ Age Group } & \multirow{2}{*}{ Overall } \\
\hline \multirow{2}{*}{ Drag } & $F$ & $M$ & $<=30$ & $>30$ & \multirow{2}{*}{78.50} \\
\cline { 2 - 5 } & 82 & 75 & 73 & 84 & \\
\hline \multirow{2}{*}{ Scale up } & $F$ & $M$ & $<=30$ & $>30$ & \multirow{2}{*}{42} \\
\cline { 2 - 5 } & 46 & 38 & 27 & 57 & \multirow{2}{*}{45.50} \\
\hline \multirow{2}{*}{ Scale down } & $F$ & $M$ & $<=30$ & $>30$ & \multirow{2}{*}{60} \\
\cline { 2 - 5 } & 48 & 43 & 27 & $>30$ & \\
\hline \multirow{2}{*}{ Rotation } & $F$ & $M$ & $<=30$ & 73 & \\
\cline { 2 - 5 } & 77 & 43 & 47 & & \\
\hline
\end{tabular}

The results of a two-way between-subjects ANOVA on the success revealed a significantly main effect of the age group factor in the scale up $(\mathrm{F}(1,40)=8.052, \mathrm{p}=$ $.007)$, scale down $(\mathrm{F}(1,40)=10.913, \mathrm{p}=.002)$ and rotation $(\mathrm{F}(1,40)=5.930, \mathrm{p}=.020)$ tasks but not in the drag task $(\mathrm{F}(1,40)=.951, \mathrm{p}=.336)$. The statistical analysis of the gender factor only revealed a significant effect in the rotation task $(\mathrm{F}(1,40)=10.104, \mathrm{p}$ $=.003)$.

\subsubsection{Completion time}

The average of each subject's successful tasks is used to obtain the completion time aggregated by users (see Table 17). The unsuccessful tests were not included in the completion time analysis.

The conducted two-way between-subjects ANOVA on the completion time revealed significant main effects of the age group factor for the drag task $(\mathrm{F}(1,36)=7.844, \mathrm{p}=$ $.009)$ where the older group performed significantly faster. No other effects were found significant for any gesture and factor. 
Table 17. Completion time in miliseconds of each task by group.

\begin{tabular}{|c|c|c|c|c|c|}
\hline Task & \multicolumn{2}{|c|}{ Gender } & \multicolumn{2}{c|}{ Age Group } & \multirow{2}{*}{ Overall } \\
\hline \multirow{2}{*}{ Drag } & $F$ & $M$ & $<=30$ & $>30$ & \multirow{2}{*}{13755.53} \\
\cline { 2 - 5 } & 13721.63 & 13793.40 & 16922.11 & 10588.94 & \\
\hline \multirow{2}{*}{ Scale up } & $F$ & $M$ & $<=30$ & $>30$ & \multirow{2}{*}{9991.62} \\
\cline { 2 - 5 } & 9517.72 & 10402.34 & 8151.86 & 11586.09 & \\
\hline \multirow{2}{*}{ Scale down } & $F$ & $M$ & $<=30$ & $>30$ & \multirow{2}{*}{9859.62} \\
\cline { 2 - 5 } & 10109.92 & 9591.46 & 7732.45 & 11361.15 & \multirow{2}{*}{16651.61} \\
\hline \multirow{2}{*}{ Rotation } & $F$ & $M$ & $<=30$ & $>30$ & \\
\cline { 2 - 5 } & 16972.38 & 16239.20 & 17751.49 & 15681.13 & \\
\hline
\end{tabular}

\subsubsection{Accuracy}

In order to evaluate pre-kindergarten performance when accurate termination of the gestures is required, in this section the error values for each gesture by age group and gender are showed. For each task the error is calculated as the discrepancy between the reference and the manipulated elements. In the case of the drag gesture the error is measured as a distance in pixels between them, for the scale-up and down gestures the error is a percentage measuring the discrepancy of size and for the rotation gesture the error is measured in degrees as the difference between their rotation values.

On the one hand, the results show that both age groups have similar levels of accuracy when terminating the drag gesture, i.e., similar average error $(F(1,39)=.179, p=.675)$ with avg_error(drag, young $)=10.75 \mathrm{px}$ and avg_error(drag, old $)=8.86 \mathrm{px})$.

On the other hand, the results show that in the scale (up \& down) tasks the older group had almost a $50 \%$ greater precision than the younger one $(\mathrm{F}(1,72)=10.885, \mathrm{p}=.002)$ with avg_error(scales, young) $=20.84 \%$ and avg_error(scales, old) $=10.74 \%$. This is also the case for the rotation task in which older children have a 50\% greater precision $(\mathrm{F}(1,37)=6.497, \mathrm{p}=.016)$ with (avg_error(rot,young $)=29.7^{\circ}$ and avg_error(rot,young) $\left.=12.06^{\circ}\right)$. Finally, with respect to the gender factor no significant differences were found for any task.

\subsection{Discussion and future work}

There are several interesting conclusions that are obtained when considering multitouch gestures with high-levels of termination precision. Firstly, the results reveal that age is the main factor affecting the degree of success variable (RQ1) for all tasks except for the drag gesture. In this specific gesture all children, no matter what age, achieve similar high success rates ranging from $73 \%$ to $84 \%$. However, for more complex tasks such as rotations and two-finger scaling, the additional requirement of precise termination of the gesture has a high impact on less than 30 months children. Success rates decrease for this age group (27\%-47\%) and are significantly better for children aged 31 months and over (57\%-73\%). This result confirms that children start to develop their fine motor skills at this age (Nacher et al., 2015) and, therefore, 
Kindertivity: Usability and Communicability Strategies for Interactive Surfaces and PreKindergarten Children

applications requiring higher levels of precision could be designed to stretch and challenge children in the second age group. This would be in concordance with the principles of differentiated instruction (Subban, 2006). Secondly, it was also observed that girls are on average more successful than boys when precision is an issue (RQ2). These results are consistent with previous work which shows superior fine motor skills in girls (Moser \& Reikerås, 2014). However, our study only revealed significant differences for the rotation task. This is because the additional difficulty associated to the coordination of two finger contacts makes the scale-up/down tasks specially challenging for both boys and girls when precision is required (see Table 16).

In addition, if completion time is considered (RQ3 \& RQ4), no significant differences were found in terms of age nor gender for all tasks except for the drag gesture. This means that relatively challenging actions in terms of cognitive and motor skills such as the scale-up/down and rotation gestures are performed by all children at similar speeds. It was observed that the additional precision requirement in these gestures forced all children, no matter their age or gender, to perform the final phase of the interaction (contact release phase) repeatedly until the final successful completion of the gesture was achieved. This was not the case for the drag gesture (see Figure 36), specially perceived by older children (aged 31 months and over) as an easy to perform action they were able to complete with a lower number of attempts and, thus, resulting in significantly lower completion times.

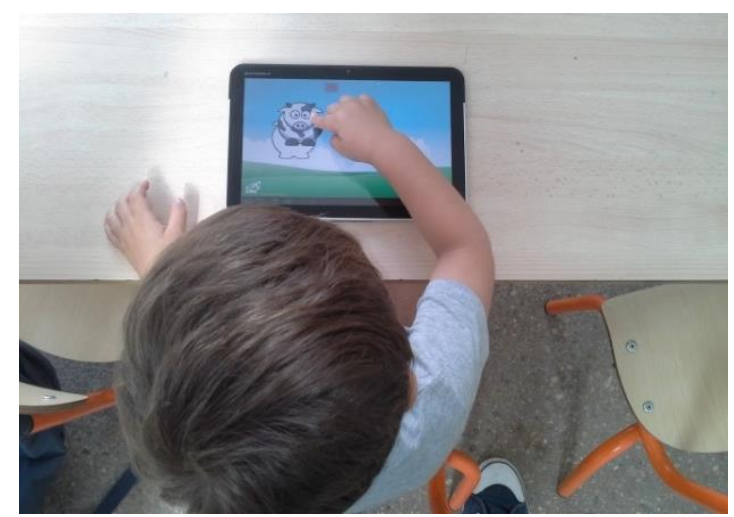

Figure 36. Pre-kindergarten child performing the drag task.

Finally, when accuracy is considered (RQ5), the analysis of the average errors reveals that the most challenging gestures (scale up-down and rotation) are performed with significantly higher levels of accuracy (lower average error) by older children (see section 7.4.3). This observation brings up the matter of using assistive strategies to deal with precision issues in an adaptive way according to the actual motor skills of each child and not, as most existing touch-based applications for pre-kindergarten children currently implement, in a comprehensive way assuming all children have the same levels of accuracy. 
The size of the objects involved in the experiments may have an impact on the effectiveness; hence, future research should be done to evaluate different sizes.

To sum up, the previous results point out that pre-kindergarten children ( 2 to 3 years old) are in the process of developing their motor skills and have different levels of accuracy. Particularly, older children are able to perform complex gestures with significantly higher levels of accuracy and, therefore, future multi-touch application for this age range should consider assistive strategies that adapt their behavior to the actual levels of motor and cognitive development of each child. Not doing so, would prevent the more skilled children from exercising and further enhancing their precision related skills at an early phase of their development.

\section{Acknowledgments}

Work supported by the MINECO (grants TIN2010-20488 and TIN2014-60077-R). 



\section{Chapter 8}

\section{Are Kindergarten Children Ready for Indirect Drag Interactions?}

Vicente Nacher ${ }^{1}$, Alfredo Ferreira ${ }^{2}$, Javier Jaen ${ }^{1}$, Fernando Garcia-Sanjuan ${ }^{1}$

${ }^{1}$ ISSI Group, Dpto. Sistemas Informáticos y Computación, Universitat Politècnica de València.

${ }^{2}$ Instituto Superior Técnico, INESC-ID/IST/Technical University of Lisbon, Lisbon, Portugal.

Published in Proceedings of the 2016 ACM on Interactive Surfaces and Spaces (ISS'16), pp. 95-101. ACM (2016)

\section{Abstract}

Several studies have shown the suitability of the direct manipulation style and the multi-touch technology for kindergarteners and, as a result, direct dragging has been established as the standard way of interaction when supporting drag manipulations for 
Kindertivity: Usability and Communicability Strategies for Interactive Surfaces and PreKindergarten Children

them. However, there are scenarios in which direct dragging is not appropriate. For example, when the object to be dragged has to move at a fixed speed or performing an animation independent of the actual direct movement speed or trajectory of the user's hand. However, nobody has yet explored the suitability of indirect dragging interaction mechanisms that allow designers to effectively implement these scenarios. This paper evaluates different mechanisms of indirect dragging manipulations (Tap-based, Accelerometer-based and Buttons-based) in order to identify those that could be used by kindergarteners in scenarios where the direct dragging is not suitable. The results show that even though kindergarten children are able to effectively use different types of indirect dragging techniques, some considerations in terms of completion time and precision must be taken into account.

\subsection{Introduction}

Children's use of interactive surfaces in distinct form-factors has become common in the last few years (Nacher, Garcia-Sanjuan, et al., 2016b). Ranging from smartphones to interactive tables and walls, these devices rely on touchscreens and are classified as natural user interfaces (NUI), often being labeled as "natural" to use. However, this might not be the case (Malizia \& Bellucci, 2012; Norman, 2010). While children appear to "naturally" interact with touch-screen devices, a great deal of attention has been given to validate this assertion. Previous studies on the usage of touchscreen devices by children have provided insightful information on this topic and point out the suitability of using the direct manipulation style concept defined by Shneiderman and Plaisant (Shneiderman \& Plaisant, 2004). Though, none has focused on the appropriateness and effectiveness of direct and indirect techniques for the dragging operation by under six-year-olds. This evaluation is relevant because, although direct dragging has been established as the de facto standard, there are scenarios in which direct dragging is not appropriate. For example, when the object to be dragged has to move at a fixed speed or performing a predefined animation (jumping or flying or affected by gravity) independently of the actual direct movement speed or trajectory of the user's hand.

In this context, we aimed to understand which approaches to the dragging operation are best suited for (pre)kindergarten children. We conducted a series of tests on a tabletsized handheld device, involving children aged from 2 to 6 years. One direct and three indirect dragging alternatives were compared. The widely used direct drag manipulation was compared against tap-based drag, accelerometer-based drag, and button-based drag.

The results of this study show the direct dragging approach as the most effective, efficient and precise technique for all (pre-)kindergarten age groups. In a context in which direct dragging cannot be applied, the most appropriate technique depends on several factors such as the effectiveness, efficiency or precision required. We believe 
these results will help developers of touch-based interfaces for children to choose the most suitable technique for each context.

\subsection{Related work}

Children's interactions with touch screen devices have recently received a great deal of attention and several studies have assessed the use of touch interaction by (pre)kindergarten children. Nacher et al. (Nacher et al., 2015) evaluated a basic set of multi-touch gestures with pre-kindergarten children ( 2 to 3 years old) and their results showed that they are able to perform basic gestures such as tap, drag, scale up, scale down and one finger rotation, even when requiring accuracy in the termination phase for some of these gestures (Nacher, Jaen, Catala, et al., 2014). Moreover, more complex gestures such as double tap and long press can also be achieved when some assistive techniques are used (Nacher \& Jaen, 2015a). Vatavu et al (Vatavu et al., 2015) also evaluated touch gestures such as tap, double tap, drag \& drop and multiple drag \& drop with children aged between 3 and 6 years old on tablets and smartphones. They concluded that, although performance increases significantly with age, all the children had high success rates when performing these gestures on both devices.

Other previous studies (Abdul Aziz et al., 2013, 2014) chose four applications and evaluated the interaction needed to play with them. Gestures such as tap, drag, rotation, drag and drop, pinch, spread and flick were tested with children aged from 2 to 4 . The authors concluded that children aged 4 were able to perform all the evaluated gestures and those aged 3 only had problems with the spread task. Finally, 2-year-olds were less effective with the more complex gestures such as drag and drop and pinch but were able to perform the tap and drag gestures properly and quickly learnt to perform the flick gesture.

These previous works reveal that direct manipulations are feasible interaction mechanisms for (pre)kindergarten children but, as pointed out in the previous section, these may not always be appropriate manipulations in some specific scenarios. To our knowledge, indirect interaction mechanisms have not yet been evaluated for these very young children. Therefore, in this paper we start addressing this issue by evaluating alternative indirect manipulations for the direct drag operation. The selection of this specific type of gesture is motivated by the requirement of some applications in which digital elements have to be moved across the surface in an indirect way.

\subsection{Experimental study}

The overall goal of our experimental study is to evaluate the suitability of different drag operations on a hand-held device and assess possible differences between them in terms of success, completion time and precision. More specifically, the research questions of the study are formulated as follows: 
Kindertivity: Usability and Communicability Strategies for Interactive Surfaces and PreKindergarten Children

RQ1: Is the degree of success independent of the drag interaction style?

RQ2: Is the degree of success independent of the age group?

RQ3: Is the completion time independent of the drag interaction style?

RQ4: Is the completion time independent of the age group?

RQ5: Is the number of collisions independent of the drag interaction style?

RQ6: Is the number of collisions independent of the age group?

\subsubsection{Drag operations overview}

The drag operations that will be evaluated in this study involve the standard direct drag operation and three indirect alternatives:

-Direct drag: Using the direct manipulation style, this natural interaction mechanism allows users to move the target element by dragging it with one finger.

-Tap-based drag: In this case, the users have to deal with a mediated interaction by tapping on the destination point to which they want to move the target, which moves in a straight line from its location to the tapped point in an animated way. While the target is moving, the point tapped is marked with a cross in order to make it visible. If the moving target collides with an obstacle or a screen border it stops.

-Accelerometer-based drag: The accelerometer is a natural interaction which allows users to move the target by tilting the device in the desired direction. As a result, the target "slides" or moves in the direction specified.

-Button-based drag: In this case four arrow-shaped buttons are used to move the target object in the four basic directions (i.e. up, down, left and right). These buttons were placed at the bottom-center of the screen (see Figure 37-Bottom). This mediated interaction mechanism allows users to move the target by tapping and holding one finger on the button that symbolizes the desired direction.

\subsubsection{Participants}

Forty-nine children (20 girls and 29 boys) from two different kindergarten schools and aged between 2 and 6 years old took part in the experiment (Mean $(\mathrm{M})=3.82$, Standard Deviation $(\mathrm{SD})=0.94)$. The children do not use touch devices in the school as a learning tool but had occasional prior experience with them in the home environment. The children were split up into three balanced age groups, i.e. they were grouped by age, with each age group $a$ comprising the ages in $[a-0.5, a+0.5[$. The distribution of the age groups is shown in Table 18.

\subsubsection{Apparatus}

The interaction framework for the experiment was implemented in Java using the LibGDX framework. The devices used for the experiment were a Samsung Galaxy 
Note 10.1 and a BQ Edison 3 tablet with Android 3.2 and Android 4.4 respectively. Both tablets equipped with capacitive multi-touch screens.

Table 18. Number of participants by age group

\begin{tabular}{|l|l|}
\hline Age group & \#Participants \\
\hline 3 & 17 \\
\hline 4 & 16 \\
\hline 5 & 16 \\
\hline
\end{tabular}

\subsubsection{Task}

A static image of an animal appears in a position on the screen and the same (reference) image appears in a white profile in another position. In order to force the children to perform drag operations which are not just simple straight lines but instead force them to change the dragging direction, either one, two or three obstacles (three difficulty levels) are placed between the two images (examples with two obstacles are shown in Figure 37). The subjects are requested to move the target to the reference image with a specific interaction mechanism (direct, tap, accelerometer or buttonbased). The task is marked as successful when the target image reaches the location of the reference image with a precision of less than 30 pixels on both the $\mathrm{X}$ and $\mathrm{Y}$ axes.

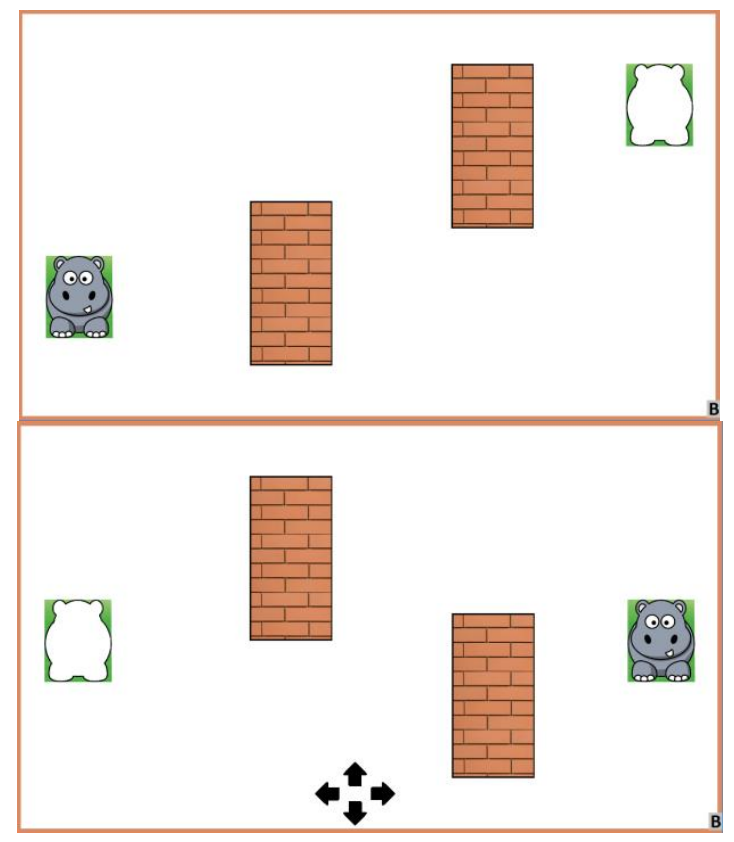

Figure 37. Example of a task with two obstacles for the drag, accelerometer, tap (top) and buttons (bottom) interaction. 
Kindertivity: Usability and Communicability Strategies for Interactive Surfaces and PreKindergarten Children

\subsubsection{Procedure}

The experiment was carried out on four consecutive days. Each day, the children performed twelve repetitions ( 4 by difficulty level) for a given task using one of the interaction mechanisms described above. The order in which the difficulty levels were presented and the order in which the interaction mechanisms were evaluated each day were randomized per subject to avoid learning effects.

For each task, the children participated in a 2-minute learning session with an instructor. Then, the experimental platform asked them to perform the task with no external adult intervention. When the gesture was successfully completed, the platform gave a positive audiovisual feedback. If the instructor observed that the participant did not carry out the task in a given time, it was marked as undone and the child went on to the next one.

\subsubsection{Design}

Three dependent variables were defined; success rate, completion time and collisions. A mixed design was made and as all the participants experimented the four interaction mechanisms, a repeated measures ANOVA (with an $\alpha=0.05$ ) was carried out with the within-subject factor Interaction mechanism with four levels (Direct vs. Tap vs. Accelerometer vs. Buttons) and the between-subject factor age group (3 years vs. 4 years vs. 5 years). A Mauchly's Test of Sphericity was carried out in order to determine whether the data met the sphericity assumptions. The tasks that did not comply with these assumptions were applied a Greenhouse-Geisser correction and were reported in the results section with F-values with fractional degrees of freedom.

\subsection{Results}

\subsubsection{Success}

In order to aggregate the success variable over the twelve repetitions, the variable was expressed as a percentage according to the number of repetitions performed successfully. The success rate of each interaction mechanism is shown in Figure 38 for each group.

The repeated measures ANOVA revealed significant differences in the success rate in the within-subject factor Interaction Mechanism $[\mathrm{F}(2.555,117.508)=34.926, \mathrm{p}<.001]$ and the between-subject factor Age group $[\mathrm{F}(2,46)=56.267, \mathrm{p}<.001]$. The post-hoc tests revealed significant differences between all the interaction mechanisms. The interaction mechanisms can be sorted by decreasing success rate as follows: Direct, Accelerometer, Tap and Buttons.

Moreover, the analysis of the Interaction Mechanism*Age group interaction revealed significant main effects $[\mathrm{F}(5.109,19.466)=56.267, \mathrm{p}<.001]$. As can be observed in Figure 38 these differences arise because there is a trend for children to be more 
successful with age in the Accelerometer, Tap and Buttons interactions. However, in the case of the Direct interaction there is no evolution in the success rates, since even the youngest group had a success rate close to 100 per cent.

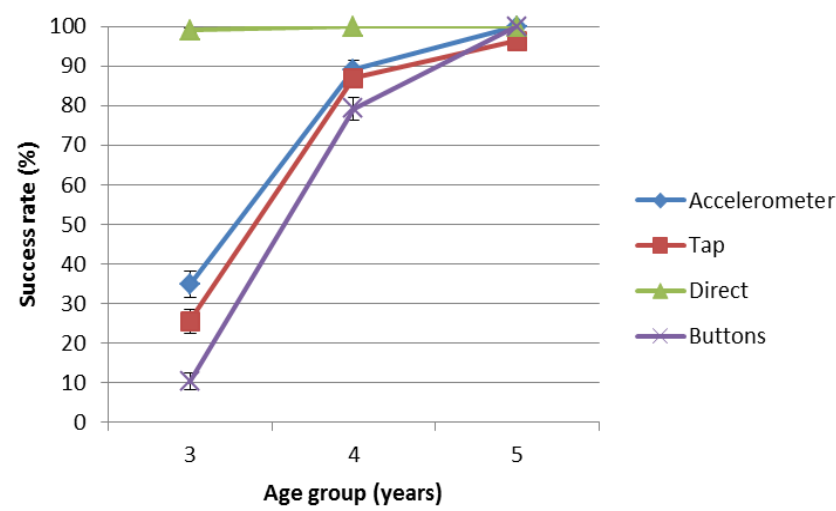

Figure 38. Success rate by interaction mechanism and age group.

\subsubsection{Completion time}

The average of each subject's successful tasks is used to obtain the completion time aggregated by users. The unsuccessful tests were excluded in the completion time analysis. The completion time is shown in Figure 39 by interaction mechanism and age group.

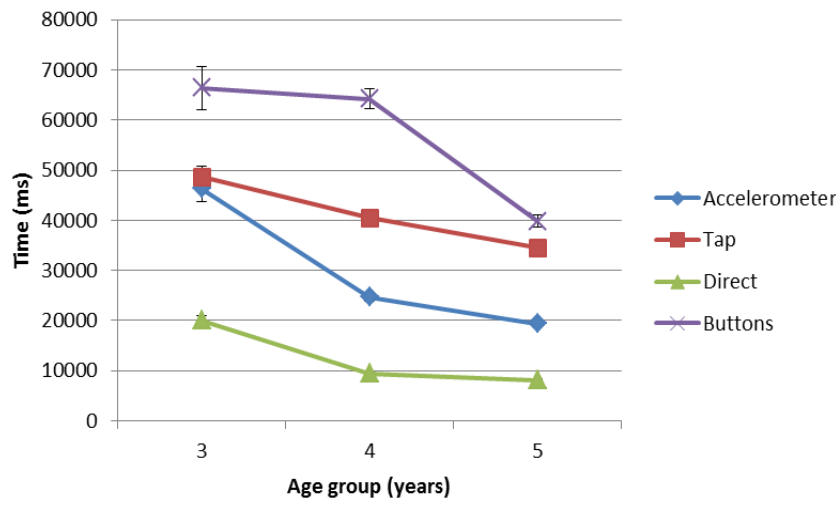

Figure 39. Completion time by interaction mechanism and age group.

Evaluating the completion time of the task, the analysis revealed significant differences in the within-subject factor Interaction Mechanism $[\mathrm{F}(3,93)=166.809, \mathrm{p}<.001]$ and the between-subject factor Age group $[\mathrm{F}(2,31)=25.674, \mathrm{p}<.001]$. The conducted post-hoc tests revealed that there are differences between all the interaction mechanisms in terms of completion time. 
Kindertivity: Usability and Communicability Strategies for Interactive Surfaces and PreKindergarten Children

The analysis of the Interaction Mechanism*Age group interaction also revealed significant main effects $[\mathrm{F}(6,93)=11.964, \mathrm{p}<.001]$. As can be seen in Figure 39, the evolution of time with age is not the same for all the interactions. In the case of the Buttons interaction there are no differences between children in the 3 and 4 year-old groups, but those in the 5 year-old group needed significantly less time to achieve the task. On the other hand, in the Tap and Accelerometer interaction there are significant differences between all age groups. Finally, in the Direct interaction children aged 4 and 5 needed less time than those aged 3 when achieving the task, but no differences were found between the two former groups.

\subsubsection{Precision}

In order to evaluate the precision when moving an object on the screen, we analyzed the number of collisions that this object had with the placed obstacles or the edge of the screen. According to this, the number of collisions by interaction mechanism and age group is shown in Figure 40.

Evaluating the number of collisions when performing the task, the analysis revealed significant differences in the within-subject factor Interaction Mechanism $[\mathrm{F}(3,99)=$ 49.594, $\mathrm{p}<.001]$ and the between-subject factor Age group $[\mathrm{F}(2,31)=25.674, \mathrm{p}<$ .001]. The post-hoc tests revealed that with the Direct interaction users had a significantly lower number of collisions and with the Accelerometer interaction users had a significantly higher number of collisions. Finally, no significant differences were found between the Tap and Buttons interactions in terms of collisions.

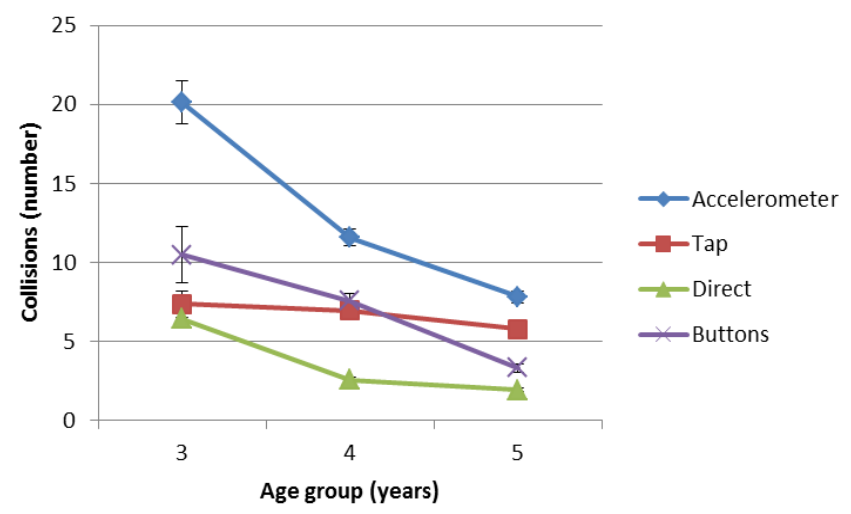

Figure 40. Collisions by interaction mechanism and age group.

The analysis of the Interaction Mechanism*Age group interaction also revealed significant main effects $[\mathrm{F}(6,99)=11.295, \mathrm{p}<.001]$. Analyzing the differences in the evolution of the collisions by age group we can see that in the Tap interaction the number of collisions remains nearly constant for all age groups. However, in the Accelerometer and the Buttons interaction there is a significant continuous 
improvement of the children's precision with age. Finally, in the Direct interaction children aged 4 and 5 were more precise than those aged 3 because the former made a significantly lower number of collisions. No differences were found between children aged 4 and 5 for the number of collisions in this interaction mechanism.

\subsection{Discussion}

In response to RQ1, the results show that the interaction mechanism used affects the success rate. As expected Direct interaction is the most effective with a $99.66 \%$ of the second most effective $(73.81 \%)$ followed by the Tap $(68.71 \%)$ interaction. Finally, the Buttons (62.07\%) interaction is the worst in terms of success. As can be seen, the two natural interaction mechanisms evaluated (Direct \& Accelerometer) overcome the two mediated interactions (Tap \& Buttons). The cognitive effort required to map mediated interactions to actual object movements have an impact at these early ages as will be discussed later.

The RQ2, on whether the degree of success is independent of the age group, is answered negatively. This is an expected result given that children are continuously developing their motor and cognitive skills. However, the degree of improvement is also affected by the nature of the interaction mechanism. This is confirmed by the results obtained related to the Interaction Mechanism*Age group statistical interaction. On the one hand, the Direct style is the less demanding and, hence, the most effective. Even the youngest children evaluated (those in the 3 years-old group) are able to perform it with success rates close to $100 \%$. Hence, there is no evolution with age, since all the children achieved the task with success rates near 100\%. On the other hand, the three indirect approaches (Accelerometer, Tap and Buttons) follow a similar evolution, with the success rate increasing with age. Our results show that the three techniques are unsuitable for children aged 3 and younger. Nevertheless, these techniques have success rates higher than $80 \%$ with children in the 4 year-old group and success rates close to $100 \%$ in the 5 years-old age group. Hence, even though these techniques are not suitable for the youngest children, they can be effectively used when the application is designed for children aged 4 or older. Finally, as can be seen in Figure 38, no differences in terms of success are found between the four interaction mechanisms evaluated with children in the 5 years-old group. At this age, children are able to achieve the task with success rates higher that $95 \%$ in all the interaction mechanisms. Therefore, our results confirm that children at this age have developed the required motor and cognitive skills to succeed when performing the evaluated indirect dragging manipulations.

RQ3 is negatively answered since the completion time is dependent on the interaction mechanism. The order from more to less effective in terms of completion time is the same as the one pointed out for the success rate. The Direct interaction is, as expected, the fastest one, since it allows children to move the target with the velocity they choose by moving their finger at the desired speed. Button-based dragging is the worst in 
Kindertivity: Usability and Communicability Strategies for Interactive Surfaces and PreKindergarten Children

terms of completion time because children need to perform repeated vertical and horizontal drag operations when a diagonal trajectory has to be followed and this is time consuming. This is not the case with the accelerometer and tap based operations, in which diagonal movements are directly supported by the interaction style. Finally, if we take a closer look at these two interactions we observe that using an accelerometer is better than using tap interactions in terms of completion time because the change of direction is easily achieved by simply tilting the tablet in the right direction (see Figure 41). In order to achieve the same effect with the tap operation, children need to move their hand across the surface to reach the desired tap point, which is less effective than tilting.

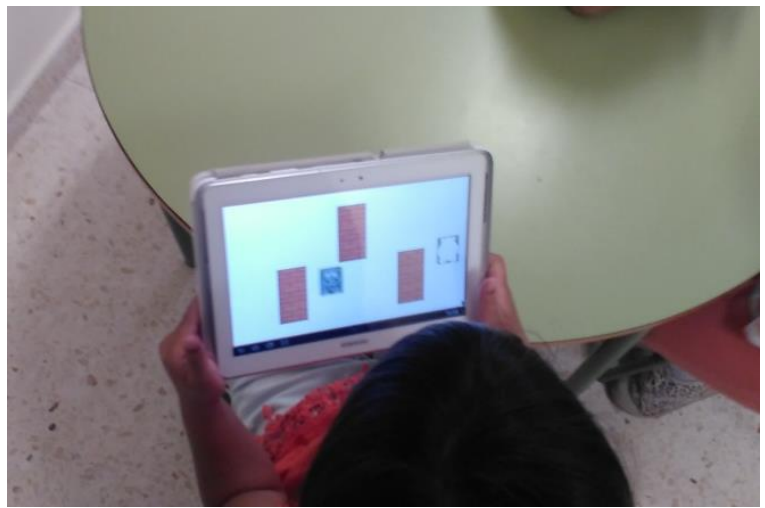

Figure 41. Kindergarten child performing the accelerometer task.

Answering RQ4, on whether age has an impact on the completion time, our results show that the required time to complete the task always decreases with age for all the evaluated interaction styles (see Figure 39). Again, as with success rates, this is consistent with the existing literature on motor and cognitive development in young children (Piaget, 1973).

Although the efficiency of all interaction mechanisms increases with age, even the most skilled children (those in the 5 year-old age group) spend a significant amount of time completing the tasks with the Buttons and Tap interaction styles, respectively. Therefore, designers of applications for kindergarteners requiring very fast indirect drag operations should implement accelerometer-based interactions instead of tap or button-based ones. In fact, the Accelerometer interaction has a significantly lower completion time with children aged $4\left(\mathrm{M}_{4 \text {-years }}=24.71 \mathrm{~s}\right)$ and children aged $5\left(\mathrm{M}_{5 \text {-years }}=\right.$ 19.34s). Of course, if direct interaction is suitable for the application being designed, it would be the best style, because of its excellent results in terms of efficiency and completion time $\left(M_{3 \text {-years }}=20.08 \mathrm{~s}, M_{4 \text {-years }}=9.43 \mathrm{~s}, M_{5 \text {-years }}=8.18 \mathrm{~s}\right)$.

Regarding the children's precision when performing the tasks, the number of collisions is dependent on the interaction mechanism and therefore the response to RQ5 is 
negative. The Accelerometer interaction is the most challenging for children in terms of precision. This occurs because kindergarten children have still not fully developed their fine and gross motor skills and, hence, they are not able to hold the device upright without tilting it. Most of the times, they tilt the device downwards and the target object collides with the bottom edge of the screen repeatedly. No differences are found in terms of collisions between the two mediated interactions (Tap \& Buttons). However, an interesting unexpected issue arose with the Tap interaction. We observed during the experiments that children do not have enough cognitive development to estimate in advance the path that the target object is going to follow as a result of a tap operation when obstacles are in their way and so they are not able to prevent the collisions. In the Buttons interaction, we also observed an interesting behavior. Kindergarten children very often held a given button until the object to be moved collided with an obstacle or the edge of the screen and then they lifted their finger and tapped another button.

Finally, regarding the effect of the age group on the precision of children (RQ6), once more the superior fine motor skills of the older age groups allows them to be more accurate when performing the task, resulting in a lower number of collisions (see Figure 40).

In addition, evaluating the Interaction Mechanism*Age group interaction, the analysis shows that in the Accelerometer interaction the number of collisions is reduced significantly with age. However, despite the reduction in the number of collisions, the most skilled children (those in the 5 years-old group) still had a considerable number of collisions $(M=7.81)$. Hence, designers should take into account that maybe this interaction mechanism is not suitable for children aged 2 to 6 in scenarios in which precision is a mandatory requirement.

Regarding the Buttons interaction, no significant differences are found between children aged 3 and 4 . However, children aged 5 are significantly more accurate than the youngest groups, with a mean of 3.32 collisions per task. Therefore, when precision is a mandatory requirement the Buttons interaction is more appropriate for children aged 5 or older.

On the other hand, no significant improvement in terms of accuracy is observed in the Tap interaction. Hence, the cognitive skills required to overcome the intellectual demand to foresee the path that the object will follow and then tap the correct location to avoid collisions is not fully developed in children younger than 6 years old. Thus, this interaction mechanism should be avoided by designers when developing applications targeted at kindergarten children in scenarios requiring high levels of precision.

Finally, in the Direct drag manipulation, children in the 3 years-old group are significantly less accurate than those in the 4 and 5 years-old group. In addition, no differences are found between the two older age groups, since these children have a 
Kindertivity: Usability and Communicability Strategies for Interactive Surfaces and PreKindergarten Children

low number of collisions $\left(\mathrm{M}_{4 \text {-years }}=2.58 \& \mathrm{M}_{5 \text {-years }}=1.93\right)$. Therefore, the Direct $\mathrm{drag}$ interaction mechanism could be used when the scenario requires accuracy in the manipulation with children aged from 4 onwards and there are no restrictions on the use of direct techniques.

In summary, in terms of design recommendations, there are several considerations to be taken into account by developers of applications for these children. First, the three indirect techniques are unsuitable for children aged 3 and younger. These children would need specific assistance strategies if any of the indirect techniques would be applied in this age range. Second, for those applications that need fast indirect dragging manipulations the recommended interaction style would be accelerometer-based followed by tap-based in case the devices to be used do not support the former interaction style. Finally, if precision is a requirement the recommended indirect dragging technique is the button-based one because, as it was discussed above, children have cognitive difficulties to estimate future trajectories of objects when using the tapbased approach.

There are some threats in terms of the generalization of the findings of this study to other contexts and environments and several precautions must be taken. The fact that children had only two minutes of training and they may have previous experiences with the direct drag technique could have influenced the results favoring it in the comparison with the other techniques. Hence, further research should be carried out in order to obtain a more comprehensive comparison between the direct-dragging and the indirect techniques.

\subsection{Conclusions and future work}

In this paper we evaluated several indirect alternative dragging mechanisms (accelerometer, tap and buttons based) with kindergarten children as potential candidate interaction styles when direct dragging operations are not suitable. These indirect mechanisms may be mandatory when implementing games or educational tablet applications for kindergarteners, in which the interactive elements to be moved across the surface have to exhibit specific behaviors which are independent of the actual direct movement speed or trajectory of the user's hand.

The results of this study show that the direct dragging approach is the most effective, efficient and precise technique for all pre-kindergarten age groups. In a context in which direct dragging cannot be applied, the most appropriate technique depends on several factors. The three indirect approaches are similarly effective, but unsuitable for three-year-olds or younger children. Indeed, while the success rate with direct dragging is close to one-hundred percent for all age groups, task completion with indirect techniques increases greatly with age. From an efficiency perspective, children under three perform faster with tap and accelerometer techniques than when using buttons, but the evolution with age is not the same for all. At age five, children are more 
efficient with the accelerometer than using tap or buttons. Regarding precision, while the accelerometer-based dragging proved worse than other techniques for all age groups, the number of collisions decreases with age for all the alternatives. We believe these results will help developers of touch-based interfaces for children to choose the most suitable technique for each context.

Our future work will evaluate the implementation of assistive techniques to overcome some of the problems related to precision that were identified in our experiments. In addition, we also plan to evaluate the ability of kindergarteners to use these indirect dragging mechanisms to move interactive characters in open 2D spaces. The design of visual semiotics (Nacher et al., 2017) to indicate to kindergarteners the direction to follow in open 2D spaces would pave the way for the implementation of educational adventure experiences in which kindergarten children could freely explore the available 2D space. In our opinion these types of games have not yet been fully exploited for this very young age range.

\section{Acknowledgments}

Work supported by the Spanish MECD (FPU14/00136), the GVA (ACIF/2014/214), and by Spanish MINECO and funded by EDRF-FEDER with Project SUPEREMOS TIN2014-60077-R. 



\section{Part III}

Communicability 



\title{
Chapter 9
}

\section{Exploring Visual Cues for Intuitive Communicability of Touch Gestures to Pre- kindergarten Children}

Vicente Nacher, Javier Jaen, Alejandro Catala

ISSI Group, Dpto. Sistemas Informáticos y Computación, Universitat Politècnica de València.

Published in Proceedings of the 9th ACM International Conference on Interactive Tabletops and Surfaces(ITS'14), pp. 159-162. ACM (2014)

\begin{abstract}
Pre-kindergarten children are becoming frequent users of multi-touch technology and, according to previous studies they are able to perform several multi-touch gestures successfully. However, they do not use these devices supervised at all times. Consequently, interactive applications for pre-kindergarteners need to convey their underlying design intent and interactive principles with respect to touch interaction. In this paper, we present and evaluate two approaches to communicate three different touch gestures (tap, drag and scale up) to pre-kindergarten users. Our results show,
\end{abstract}


Kindertivity: Usability and Communicability Strategies for Interactive Surfaces and PreKindergarten Children

firstly, that it is possible to effectively communicate them using visual cues and, secondly, that an animated semiotic approach is better than an iconic one.

\subsection{Introduction}

Recent studies (Rideout, 2011) have revealed that very young children are frequent users of multi-touch devices. In fact, children encounter touch technology often before they can even speak, they do not use these devices supervised at all times and they often want to do things on their own, instead of having their parents show them. Moreover, recent work (Nacher et al., 2015) has also revealed that children between the ages of two and four are able to perform a wider set of touch gestures than those typically used in commercial learning applications (tap and drag). In this context, a key challenge to address is the efficient and effective communication of the gestures that are expected at a given moment from these very young users, i.e., languages need to be designed for applications to convey their underlying design intent and interactive principles (Prates, de Souza, \& Barbosa, 2000) with respect to touch interaction. These languages would allow the autonomous interaction of very young children with direct touch applications without the continuous intervention and guidance of adults.

In this paper we perform a communicability evaluation to appreciate how well prekindergarten users understand the messages that communicate a given expected touch gesture using two different semiotic systems. The semiotic systems under consideration in this work are of graphical nature because, although it has already been tested that including instructions in the form of short text pieces is adequate for primary school children (Kähkönen \& Ovaska, 2006), (Niemi \& Ovaska, 2007), pre-kindergarten users do not have the required abilities to understand text messages.

The obtained results suggest that it is possible to design visual languages for communicating touch gestures for pre-kindergarten children. The animated approach that is proposed in this work is more effective to communicate dynamic gestures, i.e. gestures that follow trajectories on the screen, in terms of both intuitiveness and potential learnability.

\subsection{Related work}

The problem of communicability has received recently a great deal of attention in the context of applications for adults (Hofmeester \& Wolfe, 2012)(Walter, Bailly, \& Müller, 2013). Moreover, several works have studied the way in which instructions are given in applications for children. The work in (Kähkönen \& Ovaska, 2006) explored different ways to provide instructions in applications targeted at 5-6 year old children in desktop computers. This study resulted in some design recommendations such as giving visual cues to trigger attention to find new content, providing help in the form of text adapted to such target users, and providing separate video instructions so that 
Exploring Visual Cues for Intuitive Communicability of Touch Gestures to Pre-kindergarten Children

children can focus on the explanation given. This work also found that audio help could overcome some limitations of written instructions like in (Niemi \& Ovaska, 2007).

Another previous work (Baloian et al., 2013) has proposed a character-based language to communicate multi-touch gestures in a learning application with 5-6 year old children. Researchers associated each gesture to a specific virtual character in the learning application that appeared when a gesture was requested. The characters were chosen in such a way that the gesture was "recallable": a jumping grasshopper for a double tap, a walking ladybug for a drag gesture, a hovering butterfly for a tilting gesture and so on.

McKnight and Fitton (McKnight \& Fitton, 2010) performed an initial test of common touch-screen terminology with native English children aged between 6 and 7. Children had little or no trouble in understanding the majority of the instructions provided in both textual and audio form as they completed the task easily.

These previous works have attempted several semiotic systems consisting of text, audio and/or visual elements for very young children aged 5-7. However, there is no study that explores the more challenging pre-kindergarten age range. Therefore, in this work we explore the communicability of two languages for pre-kindergarten users so that interactive applications can effectively communicate touch gestures.

\subsection{Languages overview}

In order to adequately select the candidate languages that would be evaluated, a number of workshops took place with pre-kindergarten educators. A summary of the design rationale is described here for the two languages that were selected after this design process with pedagogy experts:

-Animated Hand language: this language uses the metaphor of a hand with one extended finger to simulate by means of an animation the required gesture. The rationale behind this language is that the object to be manipulated with a multi-touch interaction is accompanied by an animated virtual hand that provides visual cues about the gesture that should be carried out. We considered several options to visualize the form of a hand in our preliminary designs, and discussed with educators about the suitability of displaying either an isolated finger or an entire hand. Having an isolated finger was discarded and a Mickey Mouse's hand was selected by the educators for the evaluation phase (see Figure 42). For the tap gesture the hand appears and disappears once. For the remaining gestures, the hand(s) follow(s) the expected trajectory that the user's hand(s) should follow when performing the expected gesture (see Figure 43 for animated sequences).

-Iconic language: in this case the semiotic language selected by the educators consists in a static image or icon of a hand with accompanying symbolic arrows describing the 
Kindertivity: Usability and Communicability Strategies for Interactive Surfaces and PreKindergarten Children

expected gesture (see Figure 42). The icons used are extracted from a commercial icon set created to aid in the design, development, implementation and promotion of multitouch interfaces (Lee, n.d.), designed by a professional interactive designer and developer. This iconic language can also be found in Leap Motion applications. It was decided to maintain a naturalistic hand representation to evaluate this widely used commercial icon set in its original form to consider its suitability for pre-K children.

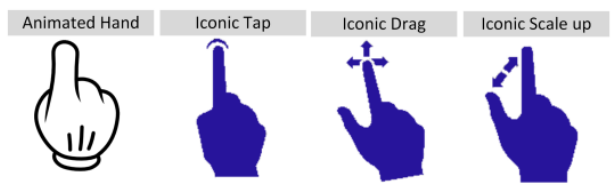

Figure 42. Evaluated semiotic elements.

\subsection{Experimental study}

The goal of this study is to obtain preliminary results about the effectiveness of two semiotic systems, one based on animations and another based on icon features, to communicate touch gestures to prekindergarten children. In this respect we have considered three categories of gestures: one-hand dynamic interactions, which require movements describing a clear trajectory; bi-manual dynamic interactions, with both hands describing trajectories; or in-place interactions, in which one hand does not actually describe a trajectory but tap at a very specific pace or in a specific way. In each category a representative gesture was selected to perform this first evaluation study: Drag, Scale Up and Tap respectively.

Therefore, the main research questions of this work are formulated as follows:

RQ1: Is any of the considered languages effective in communicating intuitively each of the considered touch gestures to pre-kindergarten children?

RQ2: Is the effectiveness of the communication process improved after a short training process?

RQ3: Is the inherent complexity of tracking several animated hands manageable?

RQ4: Is the effectiveness of the communication process affected by gender?

\subsubsection{Participants}

Parental authorization was obtained before carrying out the study. Twenty four children aged between twenty-five and thirty-eight months took part in the experiment (Mean $(M)=31.67$, Standard Deviation $(S D)=4.02$ ). The minimum age limit was chosen because children are in a pre-operational stage from 2 years old onwards. They begin to think in terms of images and symbols, and develop symbolic play with imaginary objects, which means they could be candidates for multi-touch technology at this early 
Exploring Visual Cues for Intuitive Communicability of Touch Gestures to Pre-kindergarten Children

age as discussed in (Nacher et al., 2015). Fourteen participants were female. One group by language was defined.

\subsubsection{Apparatus}

The interaction framework for the experiment was implemented in Java using JMonkeyEngine SDK v.3.0beta. The devices used for the experiment were a Motorola MZ601 and a Samsung Galaxy Note 10.1 tablet with Android 3.2 both with capacitive multi-touch screens.

\subsubsection{Procedure}

Initially, the experimenter showed each gesture without using any language (i.e. without visual stimuli associated to the gesture) and asked the children to interact to ensure that they were able to perform each gesture. This ensures that the evaluated children had the developmental cognitive and physical abilities to perform the proposed gestures.

After this activity, the participant performed an evaluation test consisting of $2 \times 3=6$ randomized trials (two repetitions of each gesture Tap, Drag and Scale up). In each test trial an image of an animal appeared on the screen (see Figure 43) and a visual stimulus describing the required gesture was displayed. The visual stimulus belonged to the language previously assigned to each subject (see Design). Once it was shown, the system awaited the user interaction without any external adult guidance. If the gesture was successfully completed, the platform gave a positive audiovisual feedback in the form of animated stars and applauses. If the experimenter observed that the participant did not carry out the gesture in less than 10 seconds, it was marked as undone and the child went on to the next trial. The system recorded the number of correct interactions. The goal of this first evaluation phase (Intuitive Phase) was to evaluate the capacity of the language to communicate a gesture without any previous language exposure.

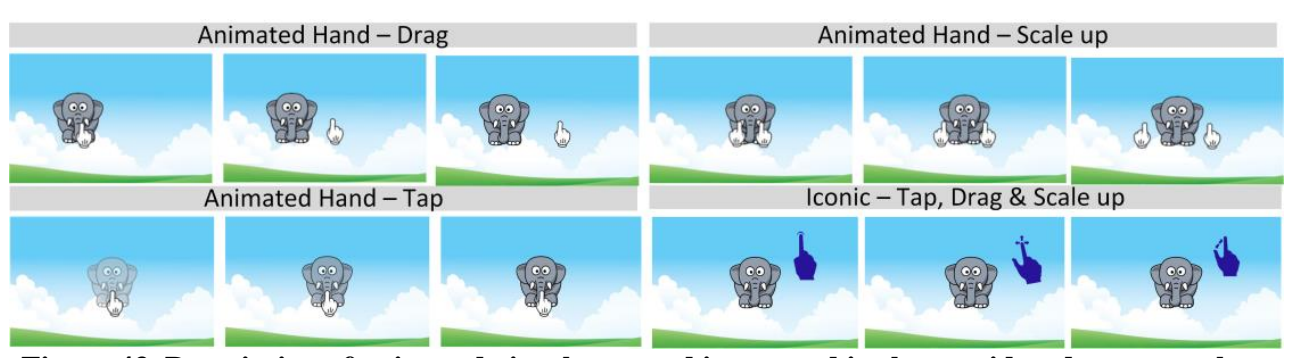

Figure 43. Description of animated visual cues and icons used in the considered gesture subset by language.

Next, the participants carried out a specific language training activity. This training activity was designed to teach children which gestures are expected to be performed given the visual stimuli provided by the language. It was carried out under the 
Kindertivity: Usability and Communicability Strategies for Interactive Surfaces and PreKindergarten Children

supervision of the experimenter who explained the visual instructions to them and the associated gestures.

Finally, an evaluation test was carried out without delay (Immediate Recall phase). In this way, this phase evaluates the impact on the overall performance of a short training session with the visual languages, i.e., to know whether the inclusion of a short guided tutorial with the proposed visual languages makes children more effective in the subsequent unsupervised interaction.

\subsubsection{Design}

Each child was only exposed to one language, which was assigned randomly at the beginning of the session. The success rate (successful interactions/total interactions) by gesture and session in a given language expressed as a percentage was obtained for each participant. This was the dependent variable used in the analysis when searching for differences between the intuitive and immediate recall phases. As data did not meet normality assumptions and given the need to handle repeated measures, the analysis was be carried out by applying the Aligned Rank Transform in order to report using ANOVA with three factors: gender, phase (Intuitive vs. Immediate Recall) and language (Animated Hand vs. Iconic).

\subsection{Results}

Table 19 shows the mean success rate for each gesture by language, gender and phase.

Table 19. Success by language, gender by phase for each task. (P1= Intuitive Phase,

\begin{tabular}{|c|c|c|c|c|c|c|c|c|}
\hline & \multicolumn{4}{|c|}{ Language } & \multicolumn{4}{|c|}{ Gender } \\
\hline & \multicolumn{2}{|c|}{ Animated } & \multicolumn{2}{|c|}{ Iconic } & \multicolumn{2}{|c|}{$\mathbf{F}$} & \multicolumn{2}{|c|}{$\mathbf{M}$} \\
\hline & P1 & P2 & P1 & P2 & P1 & $\mathbf{P 2}$ & P1 & $\mathbf{P 2}$ \\
\hline Tap & 54.2 & 62.5 & 45.8 & 54.2 & 57.1 & 64.3 & 40 & 50 \\
\hline Total & \multicolumn{2}{|c|}{58.3} & \multicolumn{2}{|c|}{50} & \multicolumn{2}{|c|}{60.7} & \multicolumn{2}{|c|}{45} \\
\hline$\overline{\text { Drag }}$ & 100 & 100 & 70.8 & 70.8 & 89.3 & 89.3 & 80 & 80 \\
\hline Total & \multicolumn{2}{|c|}{100} & \multicolumn{2}{|c|}{70.8} & \multicolumn{2}{|c|}{89.3} & \multicolumn{2}{|c|}{80} \\
\hline$\overline{\text { Scale }}$ & 70.83 & 100 & 12.5 & 4.2 & 25 & 42.9 & 65 & 65 \\
\hline$\overline{\text { Total }}$ & \multicolumn{2}{|c|}{85.4} & \multicolumn{2}{|c|}{8.3} & \multicolumn{2}{|c|}{33.9} & \multicolumn{2}{|c|}{65} \\
\hline
\end{tabular}

The ANOVA revealed significant main effects of the language factor for the drag $[F(1,48)=21.754, p<.001]$ and the scale up tasks $[F(1,48)=120.048, p<.001]$. No significant main effects were revealed for the tap task $[F(1,48)=1.594, p=.214]$. This suggests that using animated hands in dynamic gestures (i.e. those requiring trajectories) is significantly more effective than using a static iconic alternative.

The analysis revealed significant main effects of the phase factor for the scale up task $[\mathrm{F}(1,48)=6.407, \mathrm{p}=.015]$, but not for the tap $[\mathrm{F}(1,48)=0.861, \mathrm{p}=.360]$ or drag tasks 
Exploring Visual Cues for Intuitive Communicability of Touch Gestures to Pre-kindergarten Children

$[\mathrm{F}(1,48)=0.246, \mathrm{p}=.360]$. This means that a short training session has only a significant effect on the success rates of the scale up task. Moreover, for this gesture the interaction language $*$ phase was found significant $[\mathrm{F}(1,48)=15.386 \mathrm{p}<001]$, what accounts for how the performance dropped using the Iconic language, in contrast to what happened with the Animated language, which was fully learned by all the participants after only one training session.

The analysis shows significant main effects of the gender factor for scale up $[\mathrm{F}(1,48)=$ $22.074, \mathrm{p}<.001]$, with males performing more successfully (65\% vs. $33.9 \%)$. No differences were found for the tap $[\mathrm{F}(1,48)=2.648, \mathrm{p}=.112]$ and the drag gestures $[\mathrm{F}(1,48)=3.791, \mathrm{p}=.059]$.

\subsection{Discussion and future work}

In response to RQ1, the results show that the Animated Hand language has a better performance to communicate touch gestures that involve movement of contact points on the surface. This is a valuable result because developmental psychologists such as Levine and Piaget suggested that kids develop spatial reasoning during middle childhood (Levine, Huttenlocher, Taylor, \& Langrock, 1999). However, our study suggests that basic reasoning related to the interpretation of moving elements on a surface can be effectively performed during early childhood. On the other hand, differences between languages for the in-place gesture (i.e. tap) were not significant. In fact both languages resulted in equally poor performance because they were unable to convey in an intuitive way the tap operation. In our opinion, this type of reasoning is more complex because it involves a process of classification and association of a visual stimulus to a gesture whose nature cannot be interpreted in terms of spatial analogy with the stimulus. This type of reasoning, as pointed out by Piaget is developed by children at a later stage.

The results obtained after a single training session found that the success improved in general, especially for dynamic gestures, although this improvement was only statistically significant for the scale up operation. Hence, the answer to RQ2 is that a single training session is not enough to learn all the considered gestures being particularly problematic the gestures that do not involve movements on the surface.

Moreover, results showed that tracking several animated hands seems manageable, what responses affirmatively to our RQ3. The scale up task has a $70.83 \%$ success percentage without any previous explanation and reaches $100 \%$ after the learning phase.

In response to our RQ4, about whether gender would make a difference, results showed that the success was only significantly different for the gesture requiring two contact points (i.e. scale up), for which male pre-kindergarten children seem to be more effective than female ones in understanding the required gesture. This is consistent with existing preschool literature on gender differences in visual-spatial cognition 
Kindertivity: Usability and Communicability Strategies for Interactive Surfaces and PreKindergarten Children

reviewed by Levine, and colleagues (Levine et al., 1999). They found that, on average, preschool boys are more accurate than girls on spatial tasks.

The previous results suggest that designers of direct touch applications for pre-K children should include animated elements to communicate touch gestures that require moving contact points on the surface if children need extra information to proceed. However it remains to be studied more effective mechanisms to communicate static gestures such as tap, double tap and long-pressed. Although these results are promising, there are clear limitations to our work. The experiments involved only one interactive element at a time in the user interface and it remains to be evaluated the effectiveness of animated languages when the interaction area is cluttered with many touchable elements or with elements that may be manipulated with several different gestures. It also remains to be verified whether the inclusion of additional gestures with their corresponding semiotic elements will have an impact on the overall performance because pre-kindergarten children are not able to recall such a variety of different elements.

\section{Acknowledgments}

Work supported by the MINECO (grant TIN2010-20488) and GVA VALi+d program (grant APOSTD/2013/013). 


\section{Chapter 10}

\section{Evaluating Multi-touch Semiotics to Empower Pre-kindergarten Instruction with Interactive Surfaces}

Vicente Nacher, Javier Jaen, Alejandro Catala

ISSI Group, Dpto. Sistemas Informáticos y Computación, Universitat Politècnica de València.

Published in Interacting with Computers 29, pp. 97-166. Oxford University Press (2017) 
Kindertivity: Usability and Communicability Strategies for Interactive Surfaces and PreKindergarten Children

\begin{abstract}
The direct manipulation interaction style of multi-touch technology makes it an ideal mechanism for implementing learning activities for pre-kindergarten children. This is feasible because recent studies have shown that users in this early age range are able to perform a complete set of basic multi-touch gestures. However, it is still unknown whether applications based on this interaction style can use effective communication strategies that enable these very young users to understand the multi-touch nature of the interactive content that is presented to them in their everyday educational applications. This understanding is particularly important in collaborative dialogic learning scenarios in which several children may participate under the supervision of an adult instructor and in which they need to discover interactive content. In order to answer this question, this work evaluates three approaches to communicate different touch gestures to pre-kindergarten users. The results obtained show, firstly, that it is possible to effectively communicate these gestures using visual cues; secondly, that animated semiotic approaches are more effective and learnable than iconic approaches and, finally, that gender differences exist in some gestures due to visual-spatial cognition development differences.
\end{abstract}

\title{
10.1. Introduction
}

Traditional forms of media such as videos and books have proven their benefits for early childhood cognitive development (Linebarger, 2005) (Mumme \& Fernald, 2003) (Anderson, Huston, Schmitt, Linebarger, \& Wright, 2001). This is particularly the case when these activities take place with the active participation of caretakers (e.g., teachers or parents) in the form of dialogic reading (Mol, Bus, de Jong, \& Smeets, 2008) (Fielding-barnsley, 2002) (Huebner, 2000) (Lever \& Sénéchal, 2011) or joint video watching activities (Fender, Richert, Robb, \& Wartella, 2010) (Sims \& Colunga, 2005). These collaborative learning scenarios enhance engagement and overall language skills, such as; increased children's vocabulary, better retention, improved expressive and receptive language and better fictional narrative skills.

However, since the advent of computers and their use to support enhanced learning activities, very young children have been left behind because traditional interaction mechanisms based on mouse and keyboard are not a natural way of interaction for them. It was not until the appearance of touch-based devices such as tabletops and tablets that very young children had the opportunity to use these new forms of interactive media to engage in beneficial dialogic activities with caretakers or with other children. In this respect, multi-touch interaction has been reported as a more intuitive way of interaction (Smith et al., 2012) enabling even inexperienced users to interact with multi-touch tabletops intuitively (Jokisch, Bartoschek, \& Schwering, 
2011); (Mihajlov, Law, \& Springett, 2014); (Ioannou et al, 2013). As a result, recent studies (Rideout 2011); (L. Plowman, 2015) have revealed that very young children are frequent users of multi-touch devices and they are confronted with this technology even before oral communicative functions are fully developed. Moreover, recent work (Nacher et al., 2015) has shown that even children between the ages of two and four are able to perform a wider set of touch gestures than those typically used in commercial learning applications. Specifically, children are able to perform basic multi-touch gestures such as tap, drag, scale (up and down) and one finger rotation. This is even the case with more problematic gestures such as double tap and long pressed which are also feasible with some assisted strategies (Nacher, Jaen, Catala, et al., 2014). However, introducing multi-touch interaction in actual applications targeting collaborative learning scenarios with very young students presents several challenges as discussed in (Falloon, 2013). This is particularly the case of pre-kindergarten children who are in the process of early language development and need special communication strategies when using multi-touch technologies. In this respect, designers of applications need to implement adequate strategies to enable not only adults but also very young children to interpret the requested gestures at a given time. A key challenge is, therefore, the efficient and effective communication of the gestures that this special type of user must perform, i.e., languages need to be designed for applications that convey their underlying design intent and interactive principles (Prates et al., 2000) with respect to touch interaction. The importance of these languages must not be underestimated because, as pointed out by Derboven (Derboven et al., 2012), multi-touch interfaces can facilitate dialogic learning scenarios in which the dialog is centered around the learning activity itself rather than on the interactions the children are expected to perform each time. This would help caretakers to concentrate on the learning content to be acquired by the children and would prevent the continuous interference required if the children do not know how to actively participate in the collaborative learning activity. In addition, in scenarios in which several children interact with several tablets under the supervision of a single adult (i.e. a teacher) the presence of an understandable semiotic cue for both adults and children could be more effective than the supervisor being forced to guide all the required interactions for all the children. However, the current lack of a standardized semiotic system for communicating multi-touch gestures prevents very young infants from understanding the interactive nature of these new educational scenarios.

The problem of communicability has recently received a great deal of attention in the context of applications for adults (Hofmeester \& Wolfe, 2012);(Walter et al., 2013);(Limperos, Buckner, Kaufmann, \& Frisby, 2015), but the conclusions of these works cannot be extrapolated to pre-kindergarten children. Therefore, in this paper we perform a communicability evaluation of three different proposed languages in order to appreciate the understanding by pre-kindergarten users of the messages that communicate a given expected touch gesture. The three semiotic systems under 
Kindertivity: Usability and Communicability Strategies for Interactive Surfaces and PreKindergarten Children

consideration in this work are of a graphical nature because, even though it has already been shown that including instructions in the form of a short text or video clip is suitable for primary school children (Kähkönen \& Ovaska, 2006); (Niemi \& Ovaska 2007); (Van Der Meij \& Van Der Meij, 2014), pre-kindergarten users do not have the required abilities to read and understand text messages or complex verbal video instructions.

The results of the study suggest that it is possible to design visual languages for communicating touch gestures for pre-kindergarten children. We conclude that the animation-based approaches are more effective in communicating the evaluated gestures in terms of intuitiveness, potential learnability and memorability, particularly when the gestures under consideration are of a dynamic nature.

\subsection{Related work}

In the literature there are several studies that address the suitability of multi-touch technology with pre-kindergarten children. For example, the work by Nacher et al (Nacher et al., 2015) proved that children of between two and three years old are able to perform a basic set of multi-touch gestures such as tap, drag, scale (up \& down) and one finger rotation with high success rates. In addition, more complex gestures such as double tap and long pressed are also suitable when using some assisted strategies (Nacher, Jaen, Catala, et al., 2014). Vatavu et al (Vatavu et al., 2015) also pointed out that children aged three to six years old are able to perform the tap, double tap and single hand drag and drop gestures but they have some issues with the double hand drag and drop gestures, especially the youngest users. Nacher and Jaen (Nacher \& Jaen, 2015a) went a step further when they evaluated whether pre-kindergarteners are able to perform touch gestures that require movement of contacts with high accuracy at the termination of the gesture. Their results showed that pre-kindergarten children achieve high accuracy in drag and drop gestures but they have some issues with the one finger rotation and scale (up \& down). In this case, the use of assistive strategies is proposed to deal with precision issues in an adaptive way according to the actual motor skills of each child. Moreover, children's preference for educational tablet-based games has already been demonstrated (Furió, González-Gancedo, Juan, Seguí, \& Rando, 2013) because it involves a natural interaction style requiring little training (FernándezLópez, Rodríguez-Fórtiz, Rodríguez-Almendros, \& Martínez-Segura, 2013).

However, as pointed out in the previous section, despite the existence of works showing the suitability of multi-touch devices with pre-kindergarten children, there is no standard way of giving instructions related to touch interaction. In this respect, several works have studied the way in which instructions may be given in applications for children. For example, Kähkönen et al (Kähkönen \& Ovaska, 2006) have explored 
different ways of providing instructions in applications targeted at 5-6 year-old old children using a desktop computer. This study, including testing conditions with written instructions and animations, concluded that despite communicability being especially challenging in this age range, some design choices were effective in supporting the communication process. The most important recommendations of this study include giving visual cues to trigger attention in order to find new content, giving helpful instructions in a textual form specifically adapted to these target users and providing video instructions separated from the other modalities so that children can focus on a specific explanation. This study also found that providing help in the form of audio messages could overcome some of the limitations of written instructions. This experimental evidence is also given in (Niemi \& Ovaska 2007). In this work, an interaction design process is explored with 6 year-old children when instructions are given. The results of this study suggest that instructions in the form of animations to show the correct use of complex tools were better understood by children only if spoken instructions were also provided. Obviously, this result could have been expected because, as pointed out by Kähkönen et al, although children can quickly learn to use an application as they actually play with it by trial and error, applications for children cannot rely on written text to give instructions due to the lack of reading skills.

In (Baloian et al., 2013), the effectiveness of a language to communicate multi-touch gestures to 5-6 year old learners was explored. The study used words and pre-recorded audio sources to ask users to identify and perform gestures. Instead of referring to gestures as adults would (e.g. by their name), the researchers associated each gesture to a specific character in the learning application. The characters were chosen in such a way that the gesture was "recallable" (e.g. a jumping grasshopper for a double tap, a walking ladybug for a drag gesture or a hovering butterfly for a tilting gesture). However, the results showed no strong correlations between performance and the behavior of the characters that children liked or disliked the most.

McKnight and Fitton (McKnight \& Fitton 2010) performed an initial test of common touch-screen terminology with native English-speaking children aged between 6 and 7 in which children were asked to perform basic gestures from a written instruction that was also given in audio form. The results demonstrated that children at this age had little or no trouble in understanding most of the instructions, as they completed the task easily. The research also concluded that presenting instructions in both textual and audio form was useful, and that even less familiar terms such as "slide" or "swipe" were also well understood. However, the study also found that it was hard to make a consistent link between a term and a gesture on a touch-screen due to the children's different abilities, which makes instructions particularly critical in systems designed for them due to their more limited vocabulary and reading skills. 
Kindertivity: Usability and Communicability Strategies for Interactive Surfaces and PreKindergarten Children

All the above-cited works considered a combination of text, audio and/or visual cues to provide instructions in applications for children aged 5 to 7 years old, showing that children in this age range are able to know what they have to do at all times, despite having some difficulties. Nevertheless, few studies provide evidence involving prekindergarten children, and as Markopoulos and Bekker (Markopoulos \& Bekker, 2003) point out, interfaces for children should be designed according to their development stages and their actual needs. To the best of our knowledge, only the preliminary work of Nacher et al (Nacher, Jaen, \& Catala, 2014) is a first approach to exploring the communicability of visual languages to pre-kindergarten children, even though the scope is somewhat limited. In this initial evaluation two visual languages were considered to communicate only three touch gestures that respectively exemplify three categories of gestures: the tap gesture representing in-place gestures, in which one hand does not actually describe a trajectory but taps at a specific or in a specific way; the drag gesture representing dynamic gestures which require movements describing a clear trajectory with only one contact; and the scale up gesture representing dynamic two-handed interactions.

These preliminary results showed that an animated language performed better at communicating touch gestures that involve movement. This is an interesting result because developmental psychologists such as Levine and Piaget suggest that kids develop spatial reasoning during middle childhood (Levine et al, 1999); (Piaget 1973). However, the above-cited study suggests that basic reasoning related to the interpretation of moving elements on a surface can be effectively performed during early childhood. This is the case because there is a direct mapping between the visual stimulus and the gesture to be performed which even pre-kindergarten children are able to understand.

The present study extends the previous work in several ways. Firstly, we increase the number of considered gestures by also studying the communicability of the longpressed, one-finger rotation and scale down gestures, covering a wider and comprehensive range of basic touch gestures. Secondly, we assess the proposed visual languages after a short and a long training session, in order to evaluate their learnability and memorability, which are the important issues when targeting kindergarten children.

Finally, in this work we also evaluate a third animated language without the explicit representation of a hand in order to evaluate the suitability of this new type of animated language for pre-kindergarten children.

\subsubsection{Industrial perspective on the communicability of multi-touch gestures}

After reviewing the leading studies from a research perspective in the previous section, it is also important to examine the commercial perspective of multi-touch applications 
concerning communicability. To look at the current situation and trends we evaluated 100 educational applications ${ }^{5}$ randomly selected from the collection of pre-school educational applications in the Android AppStore and analyzed the types of supported gestures and the associated communicability strategies, if any.

The results of our analysis show that only $9 \%$ of the studied applications use some sort of semiotic to communicate the expected gestures with different communication strategies.

On the one hand there are applications that animate the object that may be the target of the interaction. For example, the games "Kids connect the dots" developed by Fun4kids honeybee and "Kid connects animals" developed by divmob repeatedly change the color of the object to communicate an expected tap gesture.

On the other hand there are applications that include additional interface elements in order to communicate the expected gesture. For example, "Kids Handwriting Grade 1 HWT" and "ABC touch lite" use arrows to communicate the desired direction of the expected drag gesture and "Baby learn fruits" uses an intermittent yellow line around the object to indicate the tap gesture. This is also the case of writing applications such as "Write abc" and "Kids learn to write" in which the required gesture is performed with an animated hand.

Other applications such as "English for kids - vocabulary" use text to indicate that a slide gesture is required, which seems to be inappropriate for pre-kindergarten children's reading skills.

The main conclusion that can be drawn from these results is that, despite the important commercial trend in developing apps for children with touch-screen technology (Nacher et al., 2015), developers do not in general use semiotic techniques to communicate the expected gestures and when these strategies are used, they are heterogeneous and of a different nature. Given the current situation, the empirical evaluation and the results obtained in this work are a step forward in the process of obtaining an effective semiotic system to communicate multi-touch gestures to prekindergarten children that may be included in most educational applications based on this technology.

\subsection{Language overview}

In order to select suitable candidate languages for evaluation, several workshops took place with pre-kindergarten educators and pedagogical experts. The design rationale of the three languages that were selected can be summarized as follows:

${ }^{5}$ Analysis of the 100 educational games http://dl.dropbox.com/u/52040030/RevisedGames.pdf 
Kindertivity: Usability and Communicability Strategies for Interactive Surfaces and PreKindergarten Children

- Animated Hand language: in this approach a metaphor of a hand with one finger extended is used to simulate the required gesture by means of an animation. The rationale behind this language is that the object to be manipulated with a multi-touch interaction is accompanied by an animated virtual hand that provides visual cues about the gesture to be carried out. We considered several options to visualize the form of a hand in our preliminary designs, and discussed the suitability of displaying either an isolated finger or an entire hand with educators. A single finger was discarded by educators because children may not understand the matching between a single finger moving and the movement of the whole hand. From the hands set presented to educators, the Mickey Mouse hand was selected over the other ones because, according to educators' opinion, the Mickey Mouse hand may be more familiar for children and it is simpler (no nails and fewer strokes). Hence, the Mickey Mouse hand was used for the evaluation phase (see Figure 44).

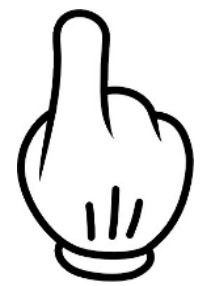

Figure 44. The image chosen by instructors for the Animated Hand language.

- Animated Object language: having seen that animated languages seem more effective than iconic ones (Nacher, Jaen, \& Catala, 2014), we designed another animated approach without the explicit representation of hands in order to evaluate its suitability.

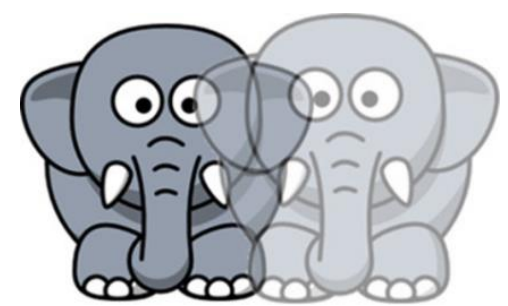

Figure 45. An example of the animated object language for the drag gesture.

In this language the object that is to be used in the interaction simulates the required gesture. The rationale behind this approach is that the object to be manipulated with a multi-touch interaction is accompanied by a shaded silhouette of itself that provides visual cues about the gesture to be carried out. For gestures that involve movement, the silhouette mimics the expected movement (see Figure 45) and for in-place ones the 
silhouette changes its shading to give information on the expected gesture (see the Tasks section for a more detailed explanation).

- Iconic language: in this case the language was selected by the educators from three commercially available iconic languages ${ }^{6,7,8}$ which serve as a control by using a language designed for adults.

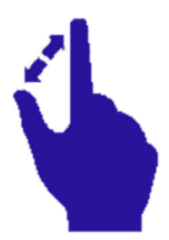

Iconic-a

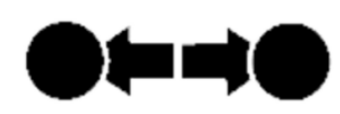

Iconic-b

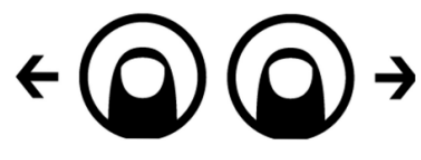

Iconic-c

Figure 46. The three available commercial iconic languages (scale up gesture).

Educators discarded the ' $b$ ' and 'c' options for being too abstract for these young users and they chose the 'a' option from Figure 46 as the best option for pre-kindergarten children. This language consists of a static image or icon of a hand with arrows to show the direction of movement (see Figure 46). Despite the selected language still maintains some symbolic representations, such as the use of arrows and a clock symbol, educators considered that it was the language with fewer abstract symbolic representations. The icons used are extracted from a commercial icon set created to aid in the design, development, implementation and promotion of multi-touch interfaces, designed by a professional interactive designer and developer. This iconic language can also be found in Leap Motion applications. It was decided to maintain a naturalistic hand representation to evaluate this widely used commercial icon set in its original form to consider its suitability for pre-kindergarten children.

\subsection{Study context}

The overall goal of our study was to test the suitability of three visual languages for communicating multi-touch gestures to pre-kindergarten children and to evaluate their intuitiveness, learnability and memorability. Two of the languages were based on animation and another was based on iconic features. Hence, using the GQM (Goal Question Metric) template (Basili et al., 1994), our goal can be defined as follows:

\footnotetext{
${ }^{6}$ Lee, R. Gestures. http://gesturecons.com/

${ }^{7}$ George, R. http://rongeorge.com/blog/gesturcons/

8 Onori P.J. http://somerandomdude.com/work/cue/
} 
Kindertivity: Usability and Communicability Strategies for Interactive Surfaces and PreKindergarten Children

analyze three visual languages for the purpose of evaluating their suitability from the viewpoint of intuitiveness, learnability and memorability in the context of communicating multi-touch gestures to pre-kindergarten children.

For this study we considered children of both genders aged between 2 and 3 years old. According to developmental theories, children are continuously developing and refining their motor skills between 2 and 7 years of age (Piaget 1973). As we were interested in exploring how gestures are learned and performed by very young children, we had defined two age groups, children younger than 30 months and children of 30 months or older. We were also interested in finding out whether certain languages should be focused on a specific target gender.

Consequently, the research questions of this work may be formulated as follows:

- RQ1: Is any of the considered languages effective in communicating each of the considered touch gestures to pre-kindergarten children?

- RQ2: Is the effectiveness of the communication process improved after a training process?

- RQ3: Is any of the considered languages more learnable than the others after the training?

- RQ4: Is the effectiveness of the communication process affected by gender?

- RQ5: Is the effectiveness of the communication process affected by the interaction of gender and language?

- RQ6: Is the effectiveness of the communication process affected by age?

- RQ7: Is the effectiveness of the communication process affected by age and language interaction?

- RQ8: Is the effectiveness of the communication process affected by the gender and age interaction?

\subsubsection{Participants}

Thirty two children aged from twenty-five to thirty-nine months took part in the experiment $($ Mean $(M)=32.875$, Standard Deviation $(S D)=3.80)$ in a balanced gender distribution of 15 males and 17 females. The participants were split up as follows; 12 interacted with the Animated Hand language (6 males and 6 females), 12 interacted with the Animated Object language (6 males and 6 females) and 8 interacted with Iconic language ( 3 males and 5 females). The iconic language had fewer subjects because some children were either not in the class every day during the evaluation or they did not want to participate in any of the sessions.

The age range of between 2 and 3 years from two Spanish nursery schools was chosen in order to explore how gestures are acquired and performed by children in the earliest 
stage of development. This age range corresponds to the first early childhood Spanish education program between the ages of one and three. The starting age of our study at two coincides with the time when fine-grained motor skills start to be developed (Piaget 1973). Parental authorization was obtained before carrying out the study.

\subsubsection{Equipment}

The interaction framework for the experiment was implemented in Java using JMonkeyEngine SDK v.3.0beta. The devices for the deployment and the experiment were a Motorola MZ601 and a Samsung Galaxy Note 10.1 tablets with Android 3.2 and Android 4.1.2 respectively.

\subsubsection{Procedure}

Educators advised us to carry out the first phase without giving children preexperimental explanations or pre-exposing them to the languages to evaluate intuitiveness and then to do several repetitions with a certain periodicity in order to evaluate the memorability of the languages. Finally, after several iterations with educators, the experiment consisted of five sessions in a period of seventeen days (see Figure 47).

In the first session, the experimenter showed the gestures without using any communication language (i.e. without visual stimuli associated with the gesture) and asked the children if they were able to perform each task to ensure that they had developed the appropriate cognitive and physical abilities to perform the proposed set of gestures.

They then performed an evaluation test consisting of $2 \times 6=12$ randomized trials (two repetitions of each gesture tap, long pressed, drag, one finger rotation, scale up and scale down). In each test an icon of an animal appeared on the screen (see Tasks section) and a visual stimulus of the required gesture was displayed. The visual stimulus belonged to the language previously assigned to each subject (see Design). The system then waited for the user interaction without any external adult guidance. If the gesture was successfully completed, the platform gave a positive audiovisual feedback in the form of animated stars and applauses. If the experimenter observed that the participant did not initiate the correct gesture in less than 10 seconds, it was marked as undone and the child went on to the next test. The system recorded the number of correct interactions. The goal of this first evaluation phase (Intuitive Phase) was to evaluate the capacity of the languages to communicate a gesture without any previous language exposure.

Once the intuitive test was performed, participants carried out a specific language training activity designed to teach children the gestures to be performed given the 
Kindertivity: Usability and Communicability Strategies for Interactive Surfaces and PreKindergarten Children

visual stimuli provided by the assigned language. This was done under the supervision of the experimenter who explained the visual instructions and the associated gestures.

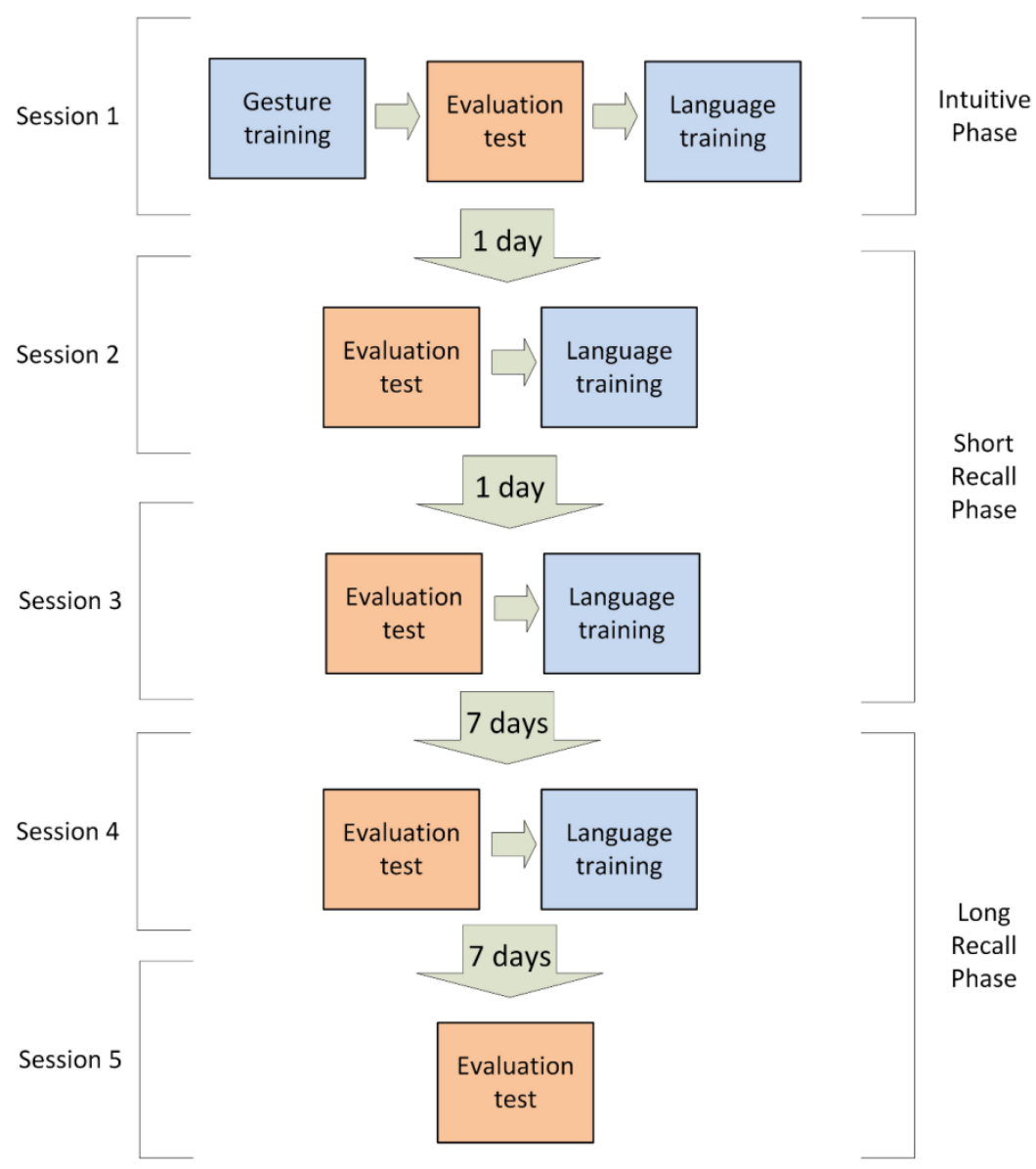

Figure 47. Details of procedure by session.

The second and the third sessions (separated by a day) consisted of an evaluation test and a language training activity to refresh forgotten gestures and strengthen the already known ones. The goal of these evaluations (Short Recall Phase) was to evaluate the learnability of the languages after a short training process.

Seven days later, the fourth session consisting of an evaluation test and a language training activity took place. Finally, after another seven days, the fifth session took place, which consisted of an evaluation test only. The goal of these two evaluations 
(Long Recall Phase) was to evaluate the learnability of the languages after a more extended training process and the memorability of the languages when a more extended period of time is considered.

\subsubsection{Design}

Each child was only exposed to one of the three languages, which were assigned randomly at the beginning of the first session. The success rate (successful interactions/total interactions) by gesture and session in a given language expressed as a percentage was obtained for each participant. This was the dependent variable used in the analysis when searching for differences between the intuitive, short and long recall phases. A mixed design was made and given the need to handle repeated measures, a repeated measures ANOVA (with an $\alpha=0.05$ ) was carried out with the within-subject factor phase with three levels (Intuitive vs. Short Recall vs. Long Recall) and the between-subject factors: gender (Male vs. Female), age group (younger or equal than 30 months vs. older than 30 months) and language (Animated Hand vs. Animated Object vs. Iconic). A Mauchly's Test of Sphericity (Mauchly, 1940) was carried out for each task in order to determine whether the data met the sphericity assumptions. The tasks that did not comply with these assumptions were applied a Greenhouse-Geisser correction (Abdi, 2010). These tasks will be reported in the results section with Fvalues with fractional degrees of freedom.

\subsection{Tasks}

This section describes the evaluated tasks and illustrates the visual stimuli for each task and language (see Figure 48 to Figure 53).

\subsubsection{Task 1: Tap}

An image of an animal appears in the center of the screen and participants are requested to tap on it in order to pass the test, with a different visual stimulus for each language. Figure 48 shows an example of communication of the tap gesture for each of the three languages. The first strip at the top of Figure 48-top shows a sequence of screens illustrating how the visual stimulus changes over time in the Animated Hand language. In this case, it displays a flashing Mickey Mouse hand next to the animal which becomes brighter with time (Figure 48-top). The second strip, Figure 48-center, illustrates the sequence for the Animated Object language. It shows a flashing animal becoming brighter from its initial state but in this case without the accompanying hand. The iconic language shows a static image of a hand with a tap symbol on the top, as illustrated in Figure 48-bottom. 
Kindertivity: Usability and Communicability Strategies for Interactive Surfaces and PreKindergarten Children

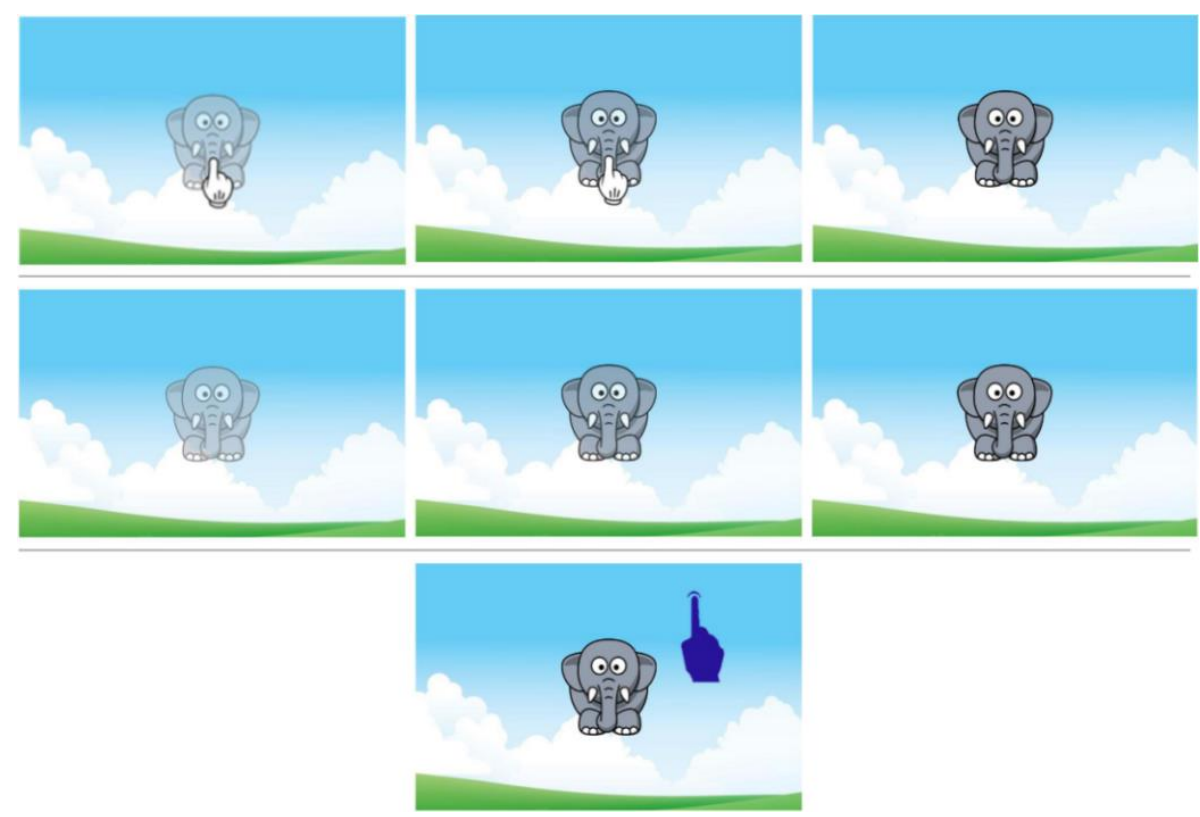

Figure 48. Examples of communication of the tap gesture for each language. Top: Animated Hand language; Center: Animated Object language; Bottom: Iconic language.

\subsubsection{Task 2: Long pressed}

An image of an animal appears in the center of the screen and participants are requested to carry out a long pressed gesture on it to pass the test. The task succeeds when the participants put their finger on the target image and hold it for at least 500 milliseconds. The animated hand language displays a flashing Mickey Mouse hand and meanwhile the animal is animated by progressively changing its brightness level clockwise as time passes (see Figure 49-top). The animated object language shows the same clockwise animation performed on the animal without any accompanying hand (see Figure 49-center). The iconic language shows a static image of a hand with a long pressed symbol on top (see Figure 49-bottom). 

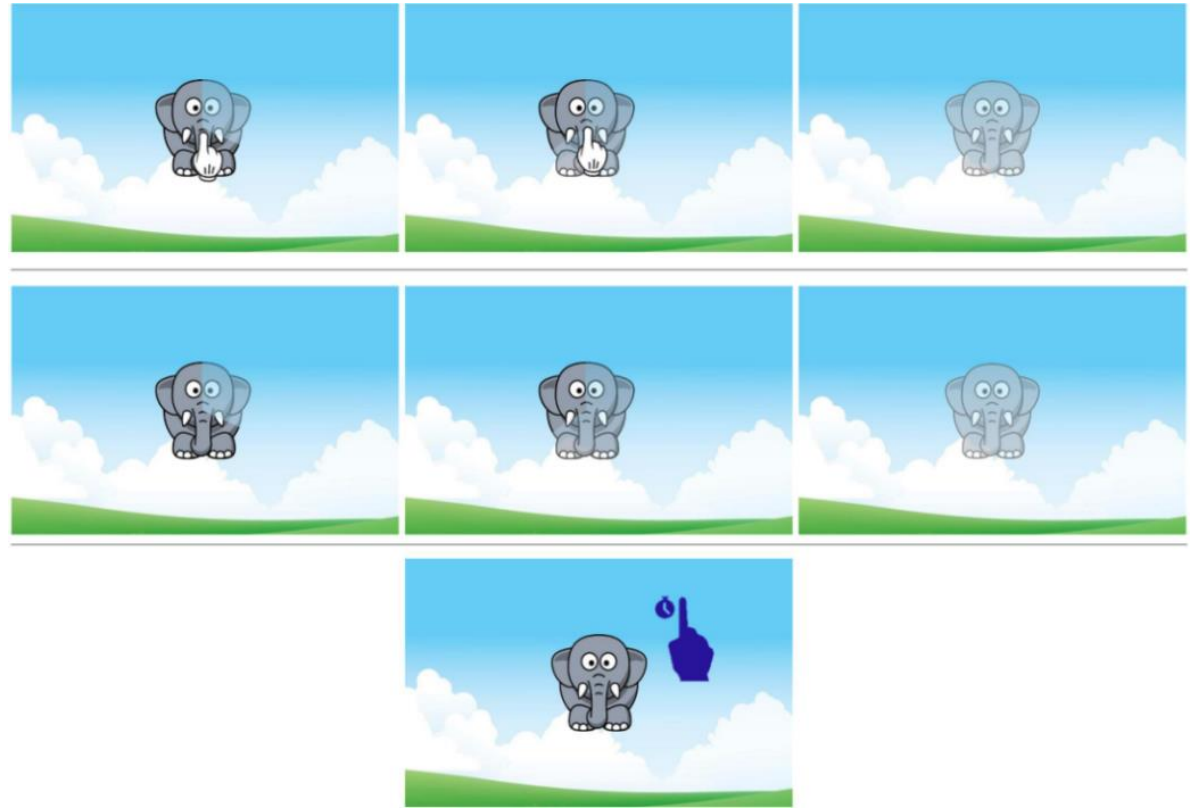

Figure 49. Examples of communication of a long pressed gesture for each language. Top: Animated Hand language; Center: Animated Object language; Bottom: Iconic language.

\subsubsection{Task 3: Drag}

An image of an animal appears in the center of the screen and participants are requested to drag the target. The task is successful if the animal is dragged at least 10 $\mathrm{cm}$. In the animated hand language the Mickey Mouse hand describes the movement expected from the user (see Figure 50-top). In the animated object language a faded version of the object describes the required drag movement (see Figure 50-middle). The iconic language shows a static image of a hand with an extended finger and arrows indicating the drag gesture (see Figure 50-bottom). 
Kindertivity: Usability and Communicability Strategies for Interactive Surfaces and PreKindergarten Children

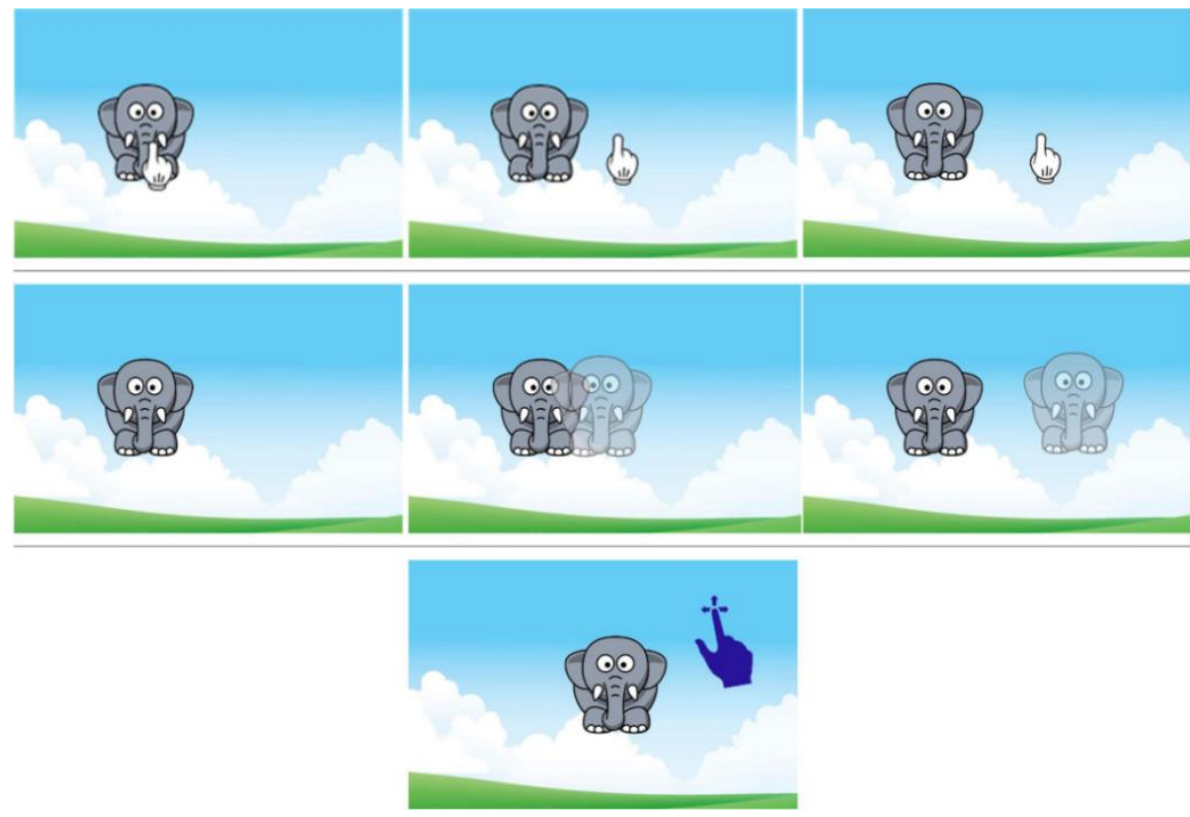

Figure 50. Examples of communication of a drag gesture for each language. Top: Animated Hand language; Center: Animated Object language; Bottom: Iconic language.

\subsubsection{Task 4: One finger rotation}

An image of an animal appears in the center of the screen and participants are requested to rotate the target image by dragging one finger around the center of the target image. Pressure can be applied on the target image itself or anywhere around it. The task succeeds when the target image reaches an angle larger than the one specified, which is automatically detected by the system to provide positive audiovisual feedback. In the animated hand language the Mickey Mouse hand describes the expected movement around the target image (see Figure 51-top). In the animated object language a faded version of the object describes the expected movement (see Figure 51-middle) and the iconic language shows a static image of a hand with an extended finger and arrows indicating the rotation gesture (see Figure 51-bottom). 


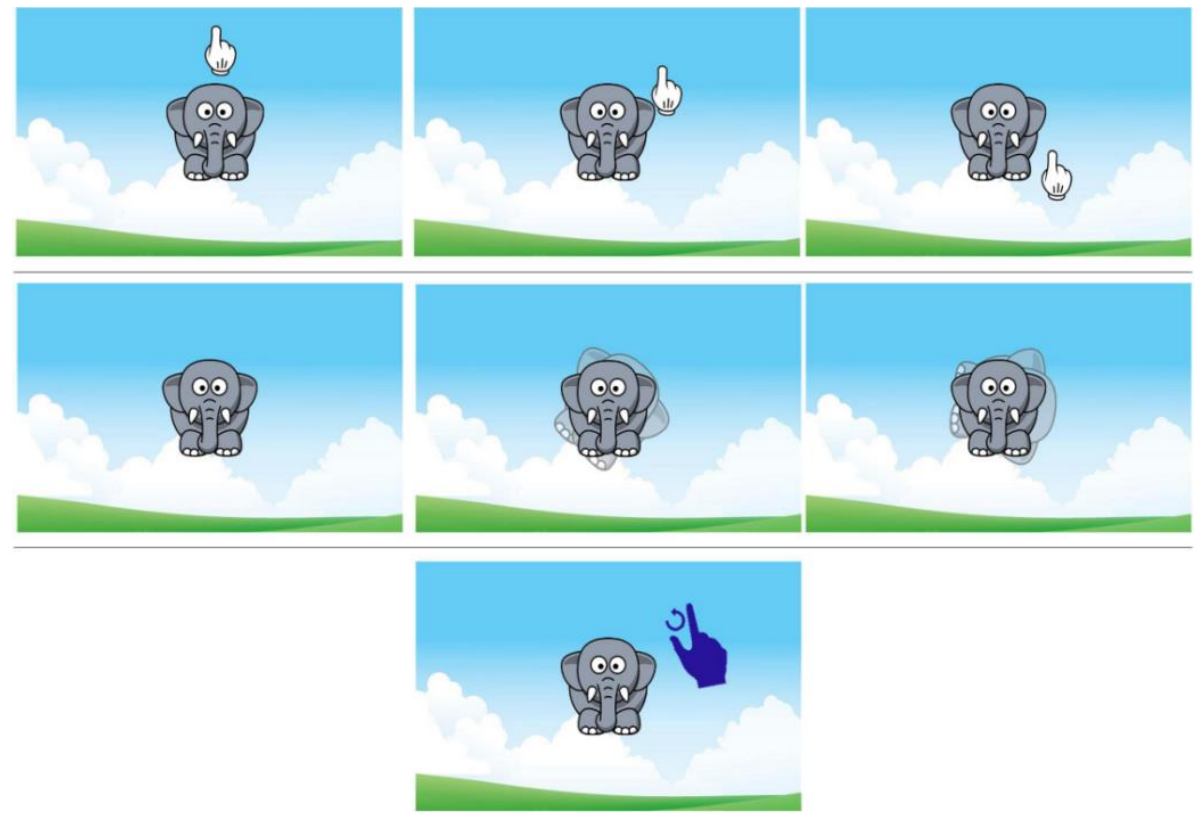

Figure 51. Examples of communication of a rotation gesture for each language. Top: Animated Hand language; Center: Animated Object language; Bottom: Iconic language.

\subsubsection{Task 5: Scale up}

An image of an animal appears in the center of the screen and participants are requested to scale up the target image 1.5 times its initial size. This can be done by expanding the distance between two fingers of either one or two hands. The fingers do not have to be in contact with the reference image and the scaling factor applied is the incremental value returned by the JMonkeyEngine runtime for this gesture. If more than two contacts are made on the surface, JMonkeyEngine considers only the two most recent ones for communicating scaling events. The task succeeds when the target image reaches the expected size, and it is not necessary for the subject to lift his/her hands when it is reached. In the animated hand language two Mickey Mouse hands describe the expected movement by moving apart from each other (see Figure 52-top). In the animated object language a faded version of the object increases its size repeatedly to symbolize the scale up gesture (see Figure 52-middle) and the iconic language shows a static image of a hand with two fingers extended and two arrows indicating the scale up gesture (Figure 52-bottom). 
Kindertivity: Usability and Communicability Strategies for Interactive Surfaces and PreKindergarten Children

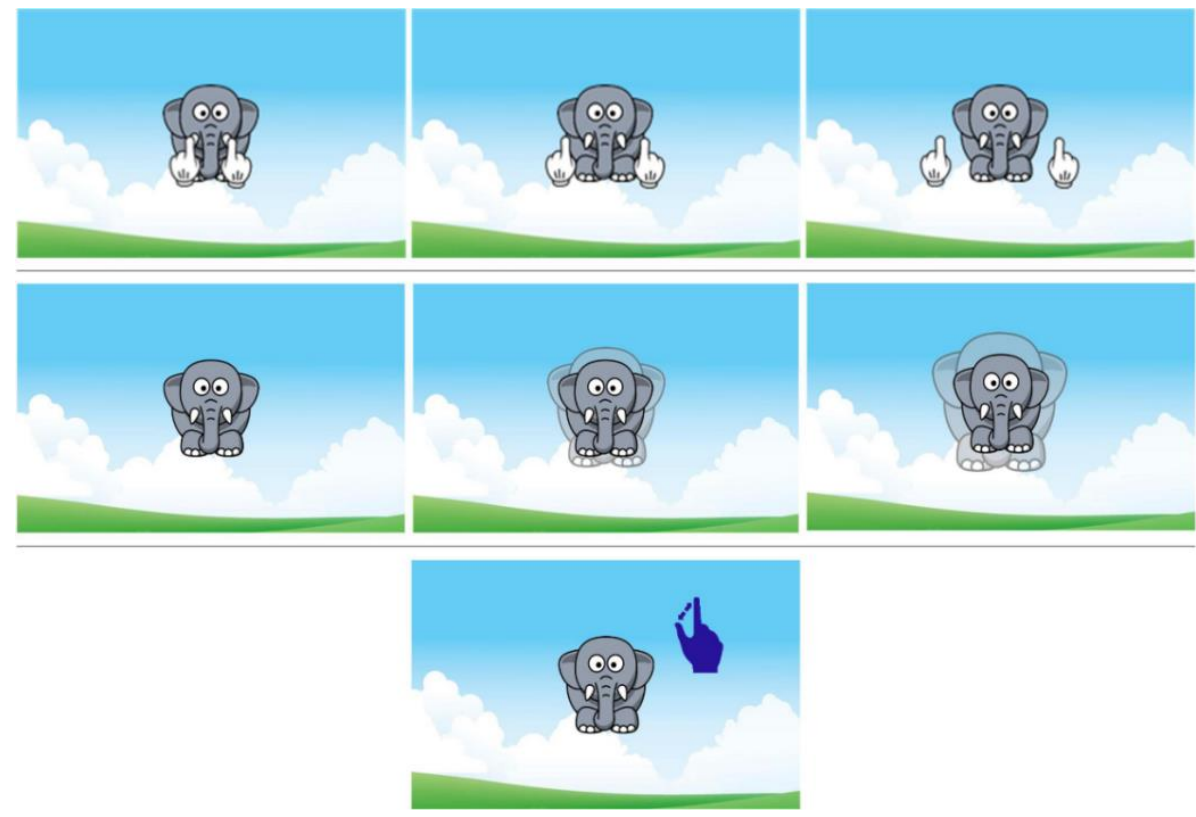

Figure 52. Examples of communication of a scale up gesture for each language. Top: Animated Hand language; Center: Animated Object language; Bottom: Iconic language.

\subsubsection{Task 6: Scale down}

An image of an animal appears in the center of the screen and participants are requested to scale down the target image by making the target object shrink until it reaches half its initial size. The task succeeds when the target image reaches the expected size with the same conditions as in the scale up task. In the animated hand language two Mickey Mouse hands describe the expected movement by moving closer to each other (Figure 53-top). In the animated object language a faded version of the object shrinks in a cyclically to symbolize the scale down gesture (Figure 53-center) and the iconic language shows a static image of a hand with two fingers extended and two arrows indicating the scale down gesture (Figure 53-bottom). 


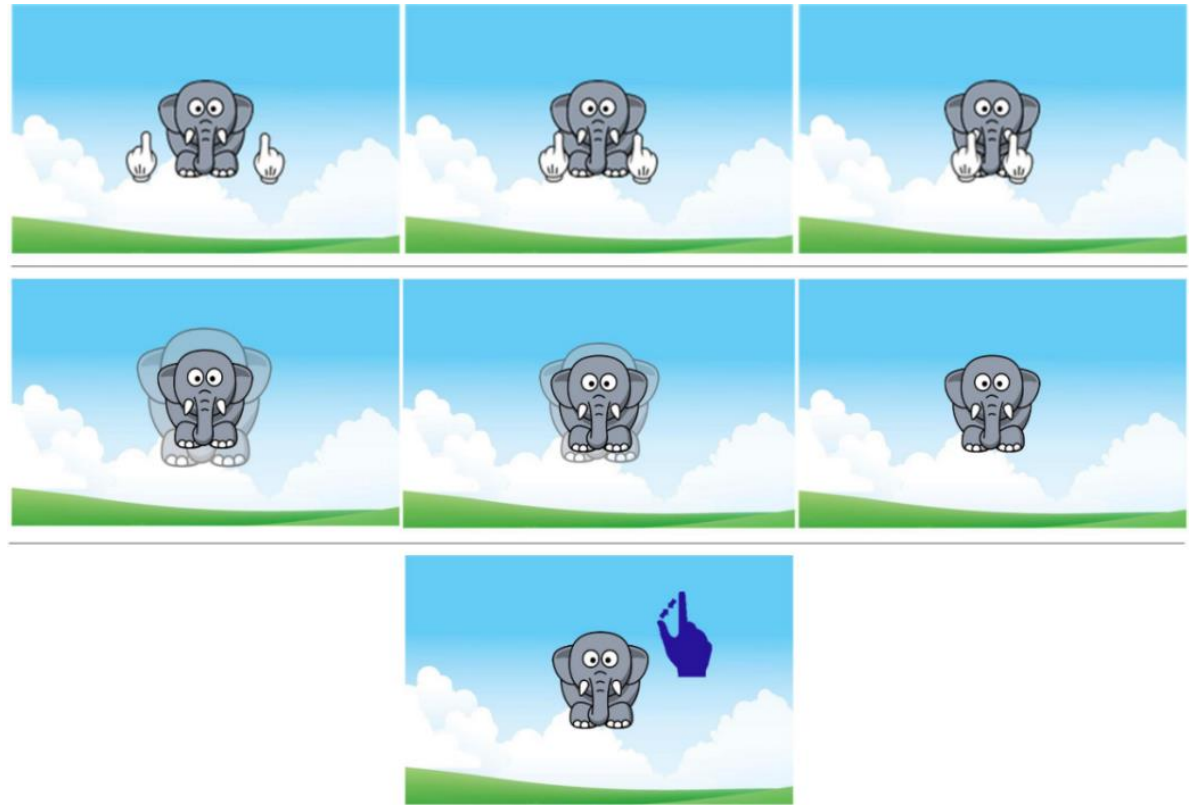

Figure 53. Examples of communication of a scale down gesture for each language. Top: Animated Hand language; Center: Animated Object language; Bottom: Iconic language.

\subsection{Results}

\subsubsection{Phases}

The collected data was aggregated by phases in order to evaluate the learnability of the languages considering the different sessions, as follows: the test in the first session was taken to be the Intuitive Recall phase; the tests in the second and third sessions were aggregated to compose the Short Training Recall phase; and the Long Training Recall phase was considered by aggregating the data of the tests from the fourth and fifth sessions. The success percentage by phase is shown in Figure 54 for each gesture, calculated as the ratio between the number of successful trials over the number of trials performed in a given condition.

The repeated measures ANOVA revealed significant differences in the within-subject factor phase for the long pressed $[\mathrm{F}(2,40)=3.651, \mathrm{p}=.035]$, the scale up $[\mathrm{F}(1.452$, $29.040)=3.931, \mathrm{p}=.043]$ and the scale down $[\mathrm{F}(2,40)=10.472, \mathrm{p}<.001]$ tasks. However, no differences were found for the $\operatorname{tap}[\mathrm{F}(2,40)=1.749, \mathrm{p}=.187]$, the drag $[\mathrm{F}(1.543,30.856)=0.756, \mathrm{p}=.446]$ and the rotation $[\mathrm{F}(1.367,27.331)=2.950, \mathrm{p}=$ .086] tasks. According to the results, the long pressed, the scale up and the scale down gestures were learned throughout the sessions and the success obtained in the long 
Kindertivity: Usability and Communicability Strategies for Interactive Surfaces and PreKindergarten Children

recall session was higher than the success obtained in the intuitive session. For the other tasks, the success level was similar in all the sessions without significant effects (see Table 20 in the Appendix for details of post-hoc tests).

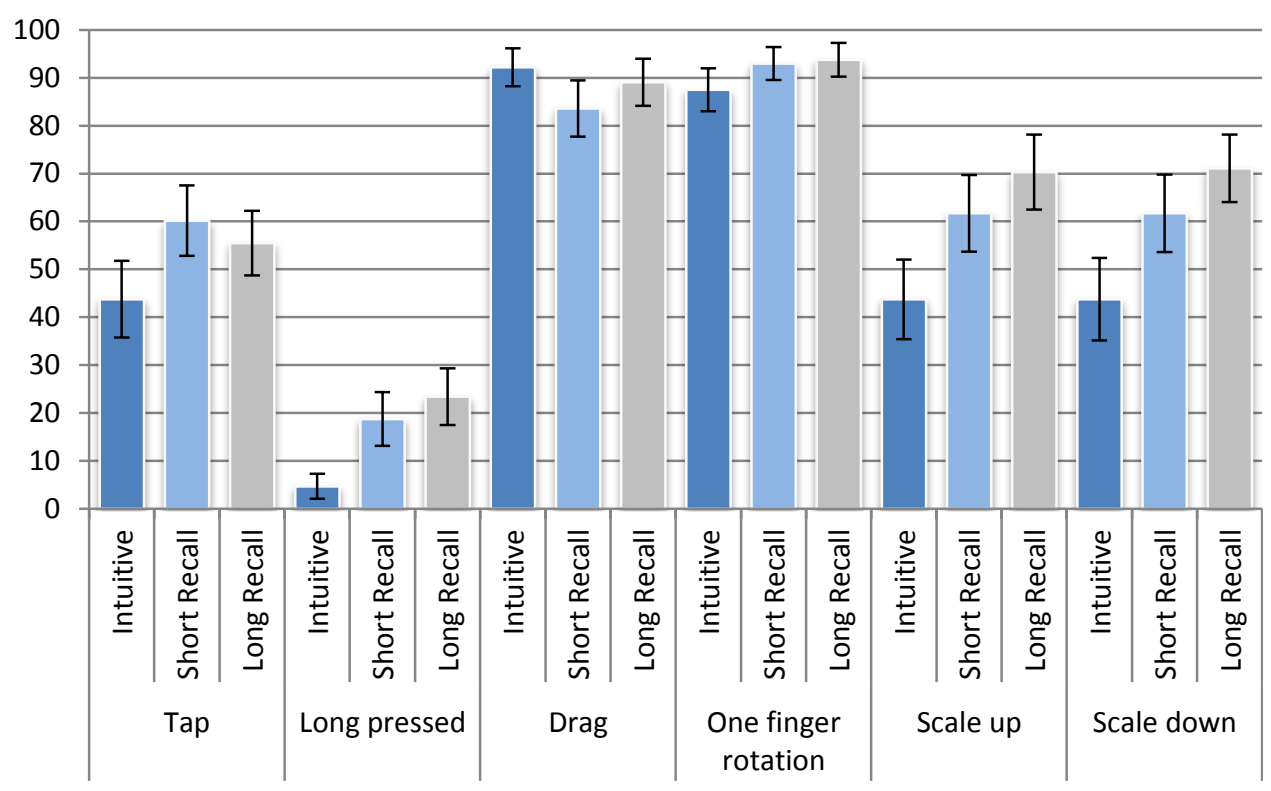

Figure 54. Success rate by phase and gesture.

\subsubsection{Languages}

In order to evaluate whether any of the languages considered is effective in communicating multi-touch gestures, the mean success rate for each gesture by language is shown in Figure 55.

Comparing the success percentage of the three considered languages, a repeated measures ANOVA revealed that the language factor is significant for the $\operatorname{drag}[\mathrm{F}(2,20)$ $=35.437, \mathrm{p}<.001]$, the rotation $[\mathrm{F}(2,20)=7.459, \mathrm{p}=.004]$, the scale up $[\mathrm{F}(2,20)=$ $25.430, \mathrm{p}<.001]$ and the scale down $[\mathrm{F}(2,20)=57.050, \mathrm{p}<.001]$ tasks. However, no significant differences were found for the $\operatorname{tap}[\mathrm{F}(2,20)=1.073, \mathrm{p}=.361]$ and the long pressed $[\mathrm{F}(2,20)=3.421, \mathrm{p}=.053]$ tasks. This means that the animated languages have better success rates than the iconic one, especially for dynamic gestures, and that there are no differences between the two animated approaches (see Table 21 in the Appendix for details of the pair-wise comparison). 


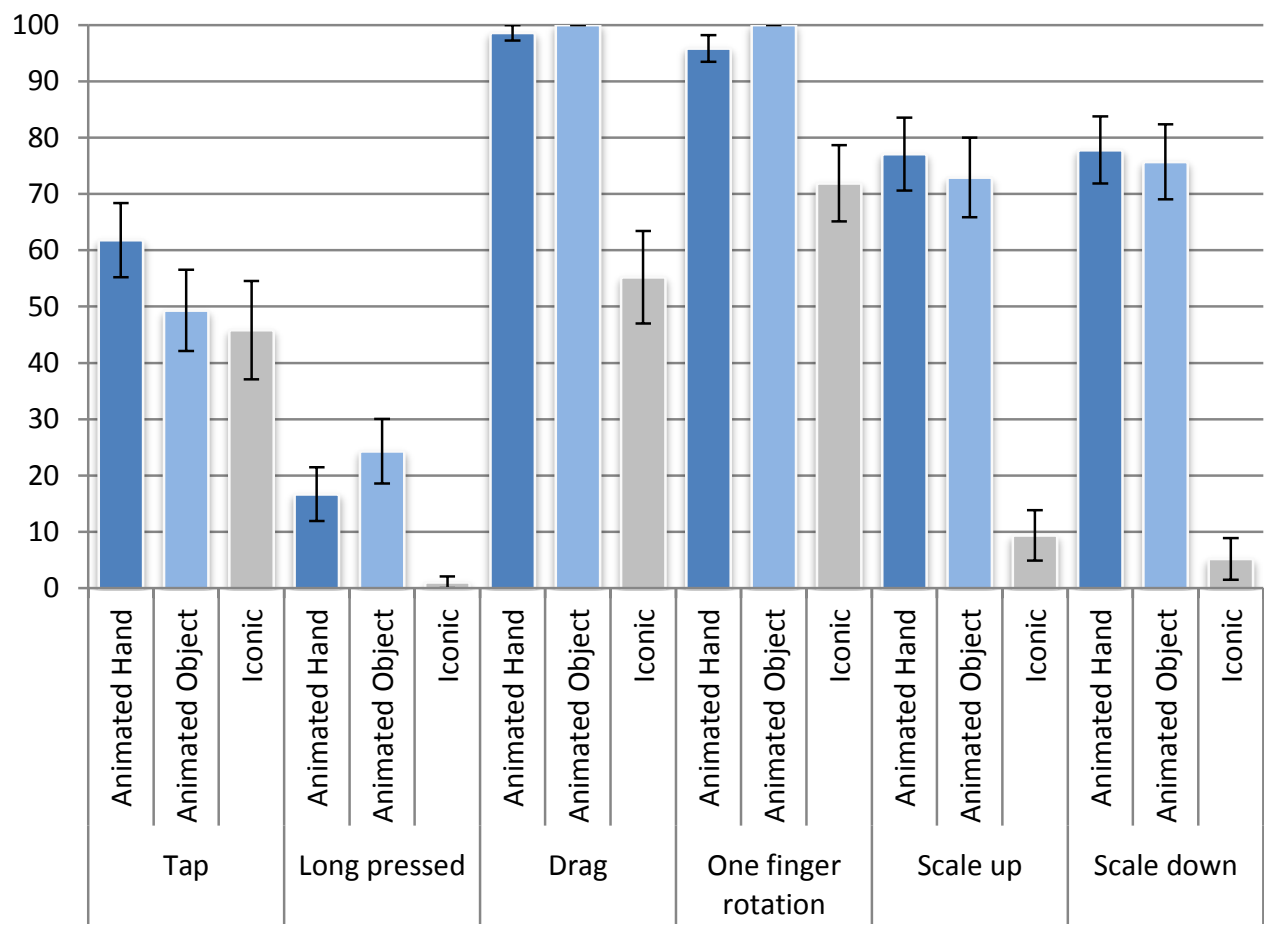

Figure 55. Success percentage by language and gesture.

\subsubsection{Phase*Language}

The results show significant differences in the interaction Phase*Language for the scale up $[\mathrm{F}(2.904,29.040)=5.535, \mathrm{p}=.004]$ and the scale down $[\mathrm{F}(4,40)=8.680, \mathrm{p}<$ $.001]$ tasks. However, no differences were found for the tap $[\mathrm{F}(4,40)=0.461, \mathrm{p}=.764]$, the long pressed $[\mathrm{F}(4,40)=.959, \mathrm{p}=.441]$, the $\operatorname{drag}[\mathrm{F}(3.086,30.856)=0.357, \mathrm{p}=$ $.790]$ and the rotation $[\mathrm{F}(2.733,27.331)=.867, \mathrm{p}=.462]$ tasks. This means that no differences were found between languages for the learnability and memorability of the in-place gestures (tap and long pressed). Moreover, the dynamic gestures that imply only one contact point (drag and rotation) are so intuitive for the animated approaches (see Figure 56) that children perform them correctly from the beginning, and so there was no evolution of the success rate. However, the iconic approach did not show a learning effect throughout the sessions, despite the lower success rate in the intuitive session (see Figure 57). Therefore, the iconic approach is not learned for gestures that involve movement of one contact point. Finally, the gestures that involve two contact points (scale up and down) were learned throughout the sessions, reaching a success rate of around $90 \%$ in the animated approaches (see Figure 56), whereas the iconic 
Kindertivity: Usability and Communicability Strategies for Interactive Surfaces and PreKindergarten Children

approach appeared as an unsuitable way of communicating these two gestures for prekindergarten children with no intuitiveness and no learning effect at all (see Figure 57).
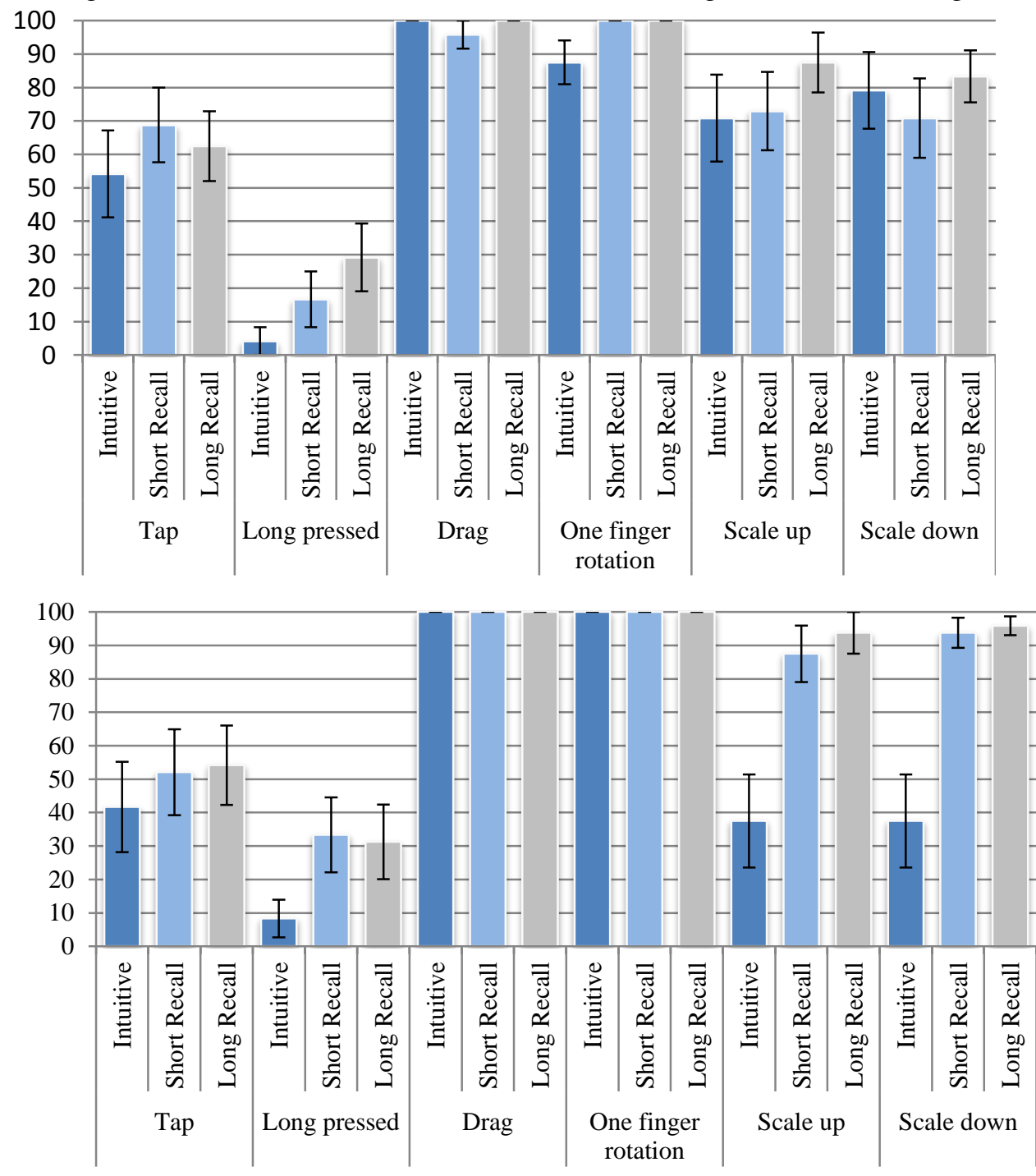

Figure 56. Success rate by phase and gesture for the animated languages. Top: Animated Hand; Bottom: Animated Object. 


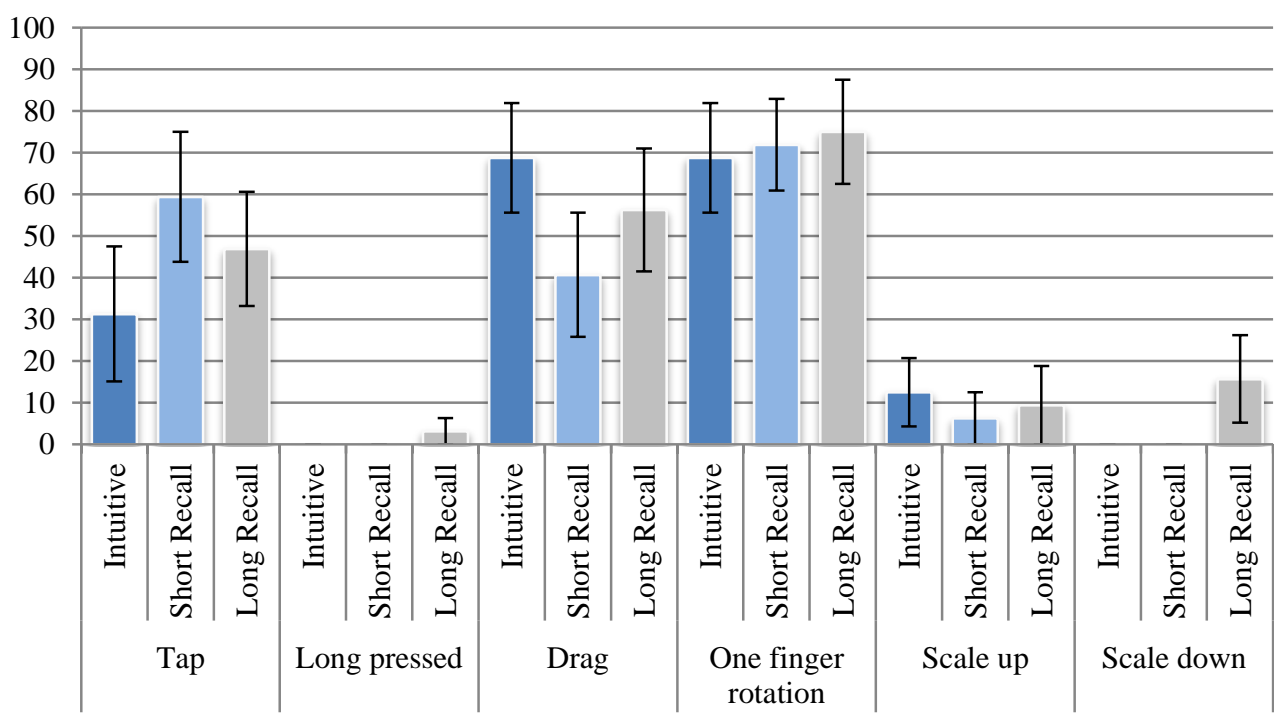

Figure 57. Success rate by phase and gesture for the iconic language.

\subsubsection{Gender}

For the gender factor the success percentage is shown in Figure 58 for males and females.

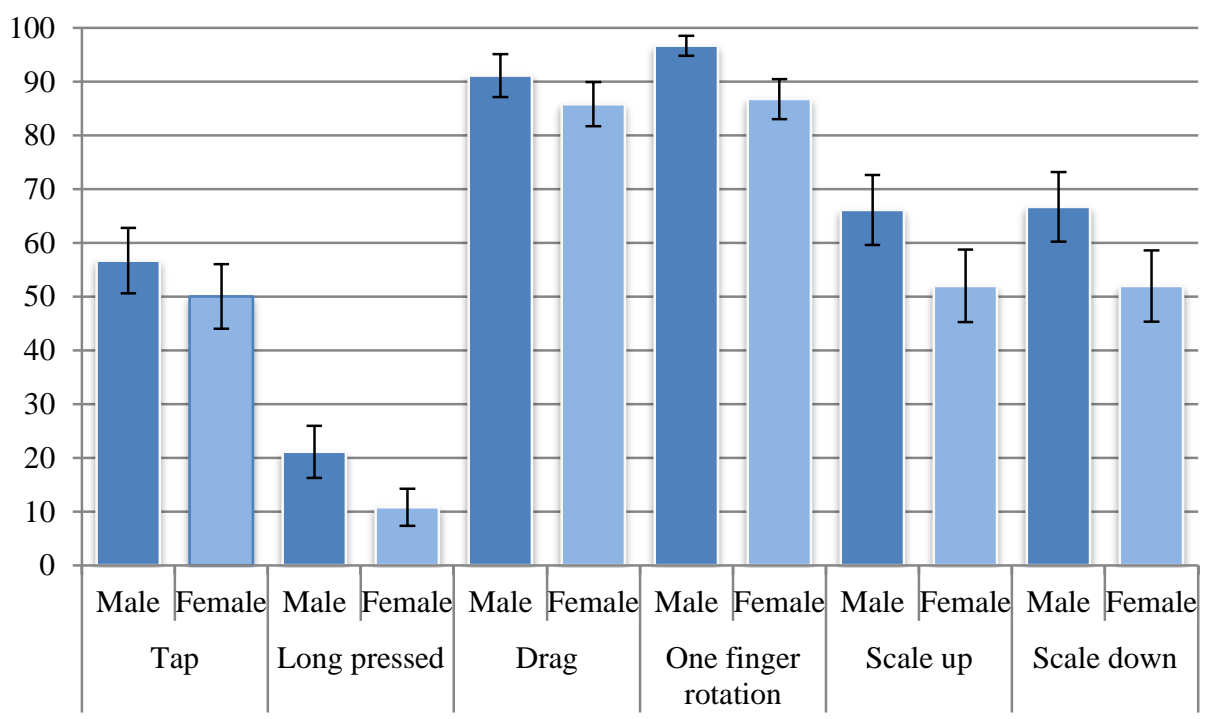

Figure 58. Success percentage by gender and gesture. 
Kindertivity: Usability and Communicability Strategies for Interactive Surfaces and PreKindergarten Children

The analysis revealed that there are no significant main effects of the gender factor for any of the tasks, tap $[\mathrm{F}(1,20)=0.231, \mathrm{p}=.636]$, long pressed $[\mathrm{F}(1,20)=0.624, \mathrm{p}=$ $.439]$, drag $[\mathrm{F}(1,20)=0.081, \mathrm{p}=.779]$, rotation $[\mathrm{F}(1,20)=3.422, \mathrm{p}=.079]$, scale up $[\mathrm{F}(1,20)=1.057, \mathrm{p}=.316]$ and scale down $[\mathrm{F}(1,20)=1.062, \mathrm{p}=.315]$. This means that there are no statistically significant differences between males and females in the success rates of the evaluated gestures.

\subsubsection{Gender*Language}

The analysis of the gender*language interaction revealed no significant differences for the tap $[\mathrm{F}(2,20)=0.408, \mathrm{p}=.670]$, long pressed $[\mathrm{F}(2,20)=0.382, \mathrm{p}=.687]$, drag $[\mathrm{F}(2,20)=0.312, \mathrm{p}=.736]$, rotation $[\mathrm{F}(2,20)=2.163, \mathrm{p}=.141]$ and the scale up $[\mathrm{F}(2,20)=3.053, \mathrm{p}=.070]$ tasks. Only for the scale down task did the analysis show significant differences $[\mathrm{F}(2,20)=4.894, \mathrm{p}=.019]$. These results revealed no significant differences between males and females in the understanding of in-place and dynamic gestures with one contact point throughout the evaluated languages. However, in the dynamic gestures which involve two contact points, male children seem to have better success rates than females in the animated languages (Figure 60) but not in the iconic approach (Figure 59). This result will be discussed later in terms of gender-related cognitive differences that may have an impact on this type of gesture.

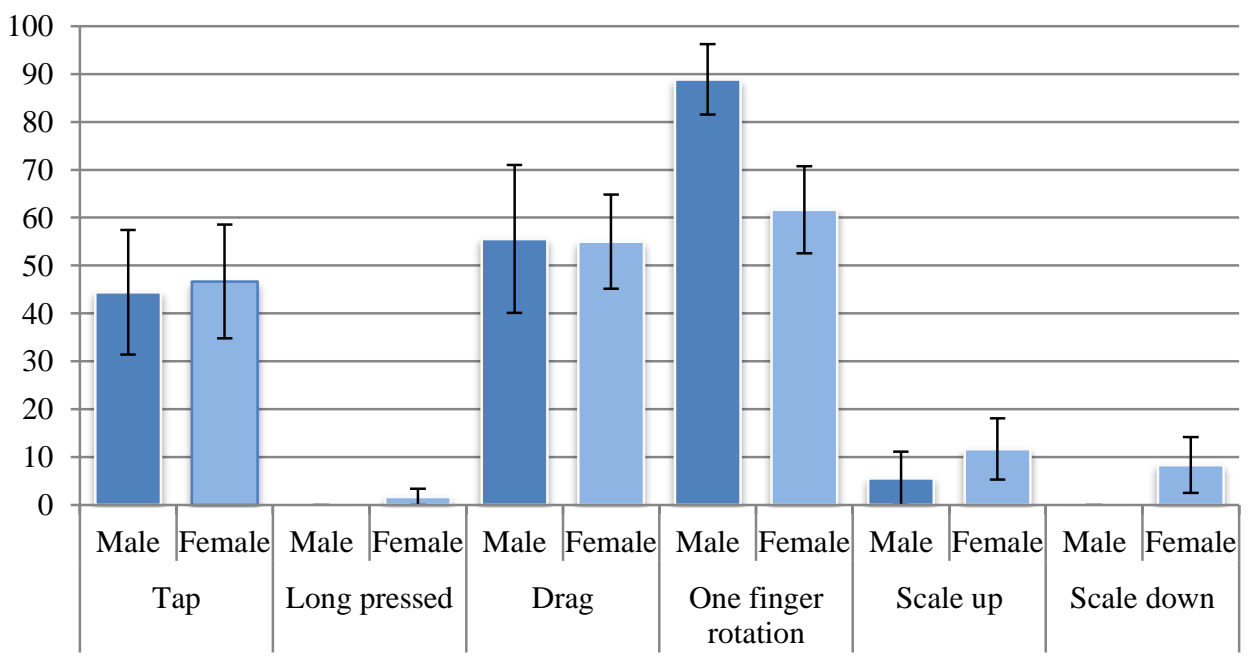

Figure 59. Success percentage by gender and task for the iconic language. 

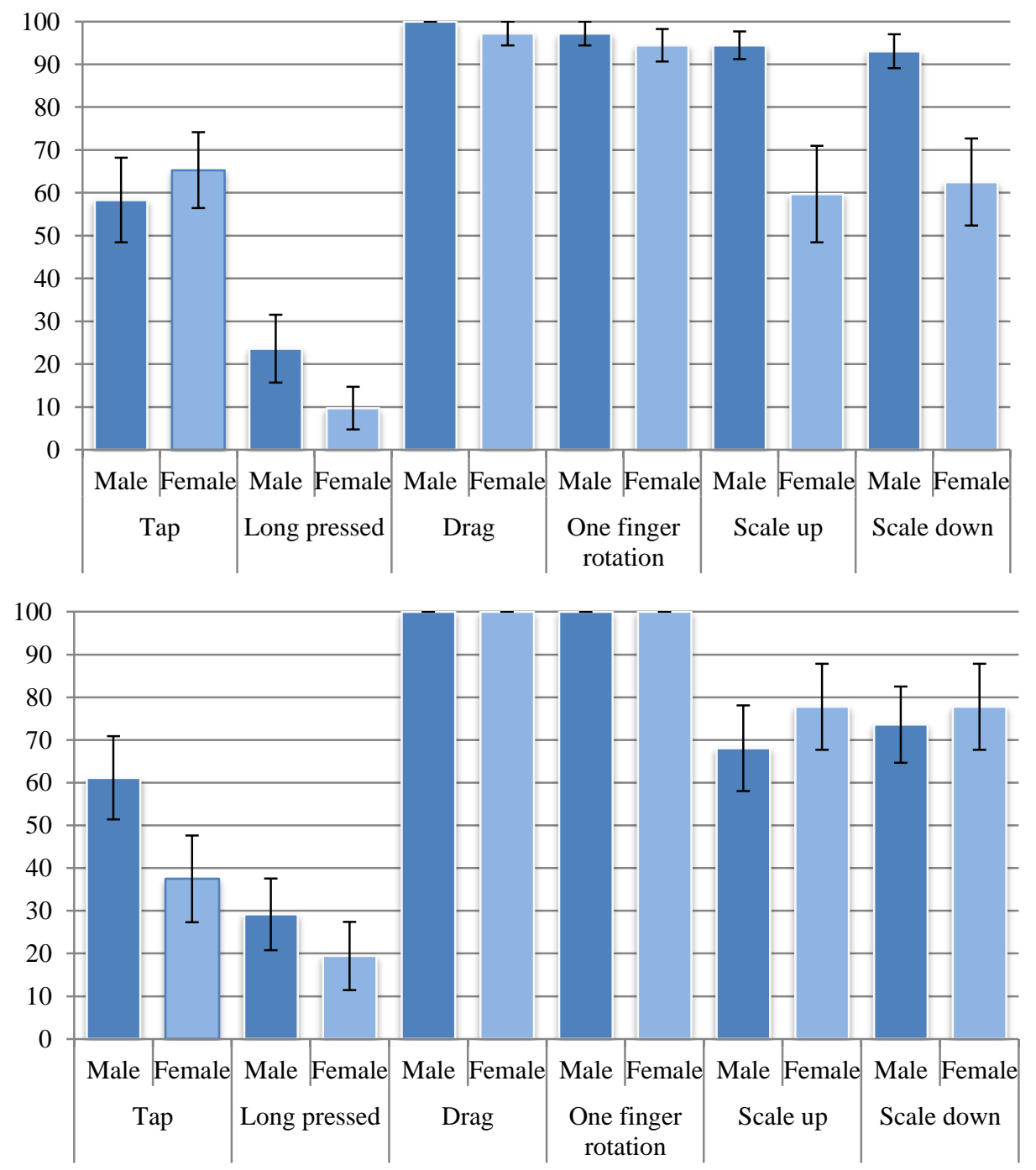

Figure 60. Success percentage by gender and task for the animated languages. Top: Animated Hand; Bottom: Animated Object.

\subsubsection{Age group}

The children were divided into two age groups (24 to 30 months and 31 to 38 months) in order to evaluate whether cognitive development of fine-grained motor skills has an impact on these two age sets. The successful percentage of tasks by age group is shown in Figure 61 for both age groups. 
Kindertivity: Usability and Communicability Strategies for Interactive Surfaces and PreKindergarten Children

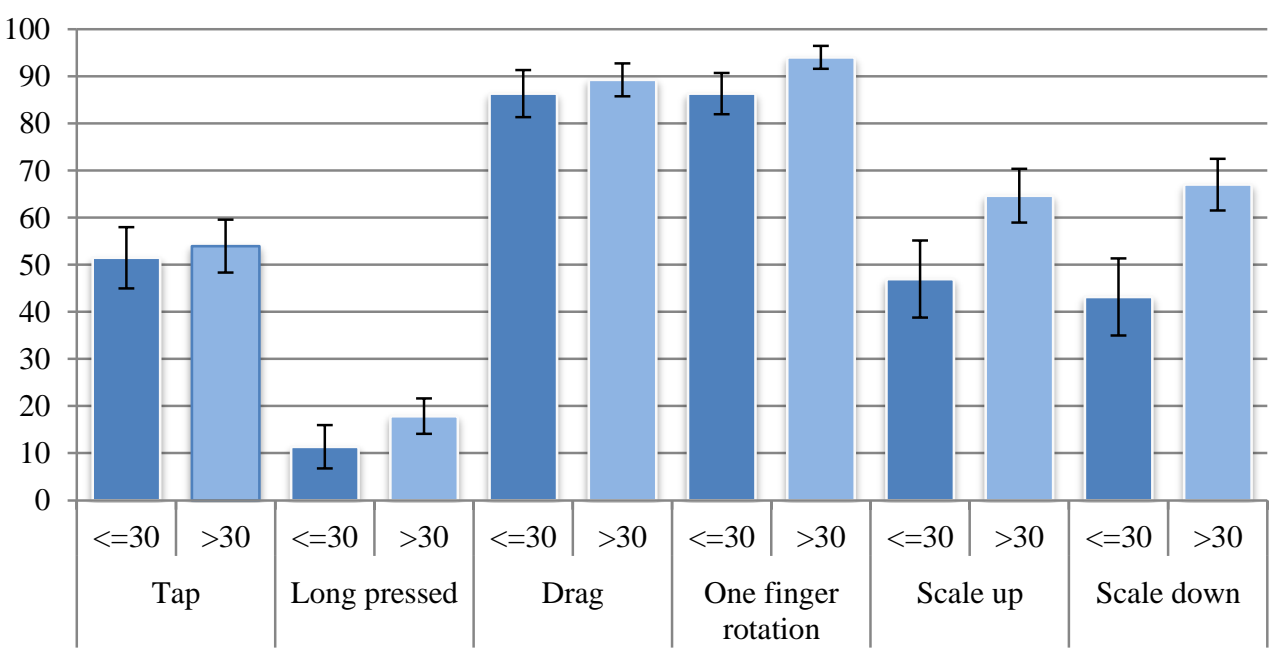

Figure 61. Success percentage by age group and gesture.

Evaluating the success percentage of the tasks by the age group factor, no significant differences were found for the tap $[\mathrm{F}(1,20)=0.124, \mathrm{p}=.729]$, long pressed $[\mathrm{F}(1,20)$ $=0.193, \mathrm{p}=.665], \operatorname{drag}[\mathrm{F}(1,20)=0.441, \mathrm{p}=.514]$, rotation $[\mathrm{F}(1,20)=1.151, \mathrm{p}=.296]$ and scale up $[\mathrm{F}(1,20)=0.565, \mathrm{p}=.461]$ gestures. However, significant differences were found for the scale down gesture $[\mathrm{F}(1,20)=4.971, \mathrm{p}=.037]$. These results show that users from the two age groups are equally successful in understanding the semiotic of both the in-place and the dynamic gestures that imply one contact point. In addition, according to the results (Figure 61) the dynamic gestures that involve two contact points are performed on average better by the older age group, although no significant statistical differences were found in the scale up task.

\subsubsection{Age group $*$ Language}

The analysis of the AgeGroup*Language interaction revealed significant main effects only for the scale up $[\mathrm{F}(2,20)=3.602, \mathrm{p}=.046]$ task and no differences were found for the tap $[\mathrm{F}(2,20)=1.104, \mathrm{p}=.351]$, long pressed $[\mathrm{F}(2,20)=0.041, \mathrm{p}=.960]$, drag $[\mathrm{F}(2,20)=0.167, \mathrm{p}=.847]$, rotation $[\mathrm{F}(2,20)=1.320, \mathrm{p}=.289]$ and scale down $[\mathrm{F}(2,20)$ $=2.759, \mathrm{p}=.087]$ tasks. This means that there are no differences on how the evolution of age affects the effectiveness of the languages in almost all of the considered gestures. Differences are only found in the scale up task, in which the older group had higher success rates than the younger in the animated languages (see Figure 62). However, this difference was not found in the iconic approach, in which the older age had no significant effect on the success rate (see Figure 63). 

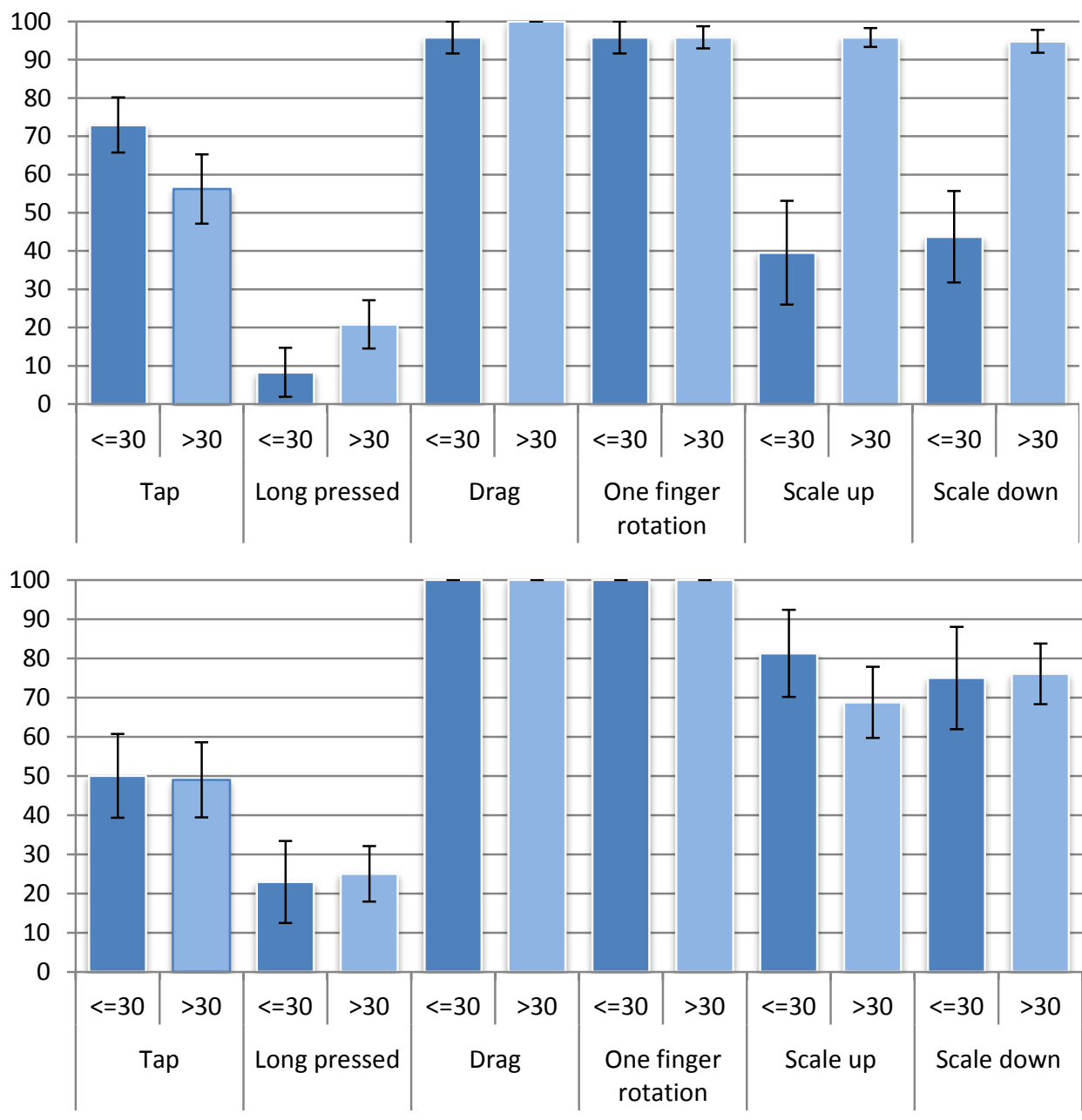

Figure 62. Success percentage by age group and task for the animated languages. Top: Animated Hand; Bottom: Animated Object. 
Kindertivity: Usability and Communicability Strategies for Interactive Surfaces and PreKindergarten Children

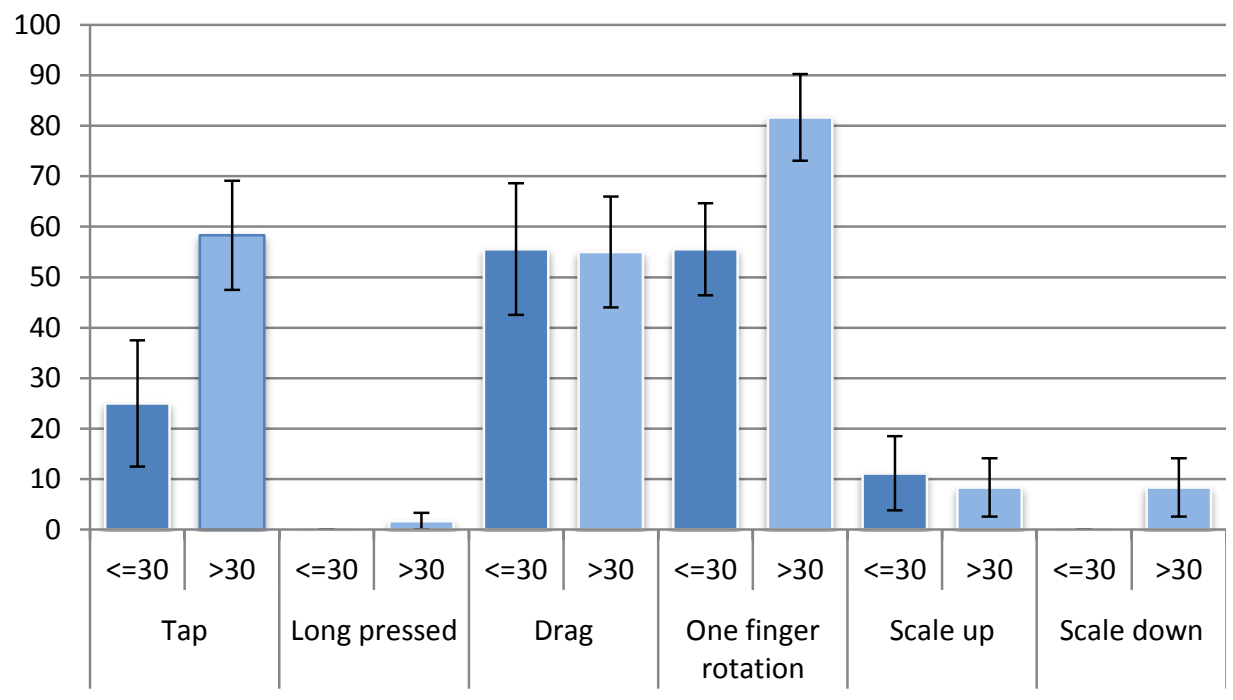

Figure 63. Success percentage by age group and task for the iconic language.

\subsubsection{Age group $*$ Gender}

The analysis of the interaction of the gender and age group factors did not reveal significant differences for the tap $[\mathrm{F}(1,20)=0.017, \mathrm{p}=.898]$, long pressed $[\mathrm{F}(1,20)$ $=0.118, \mathrm{p}=.735]$, drag $[\mathrm{F}(1,20)=2.600, \mathrm{p}=.123]$ and rotation $[\mathrm{F}(1,20)=0.629, \mathrm{p}=$ $.437]$ tasks. However, in the scale up $[\mathrm{F}(1,20)=5.491, \mathrm{p}=.030]$ and the scale down $[F(1,20)=4.482, p=.047]$ tasks significant differences were found. This means that the evolution between the younger age group to the older is not affected by gender for the in-place and one contact point dynamic gestures but the evolution is not the same for males and females for the scale up and scale down gestures, in which males in both groups had similar success rates (Scale up: $M_{\text {younger }}=68.75, M_{\text {older }}=65.15$; Scale down: $\left.\mathrm{M}_{\text {younger }}=58.33, \mathrm{M}_{\text {older }}=69.70\right)$ whereas in the case of females the younger group had significantly lower success rates than the older age group (Scale up: $\mathbf{M}_{\text {younger }}=34.52$, $\mathrm{M}_{\text {older }}=64.17$; Scale down: $\left.\mathrm{M}_{\text {younger }}=34.52, \mathrm{M}_{\text {older }}=64.17\right)$. This means that the younger girls find it significantly more difficult to perform dynamic gestures that involve two contact points.

\subsection{Discussion}

The experiment explored and answered the comprehensive set of research questions that had been posed. The answer to RQ1 (whether there can be any feasible visual languages to effectively communicate multi-touch gesture) is affirmative to a great 
extent, as the results reveal that the animated languages have better success rates than the iconic in communicating gestures that involve movement (i.e. those involving both one-contact and two contact points on the surface). This is consistent with previous results (Nacher, Jaen, \& Catala, 2014) in which the Animated Hand language was more effective than the iconic for dynamic gestures. This is an interesting result because it suggests that the direct mapping between the visual stimulus and the gesture to be performed can be effectively learned during early childhood. However, additional work needs to be done to investigate the actual nature and degree of this type of learning because developmental psychologists such as Levine and Piaget suggest that kids develop spatial reasoning during middle childhood (Levine et al., 1999). For in-place gestures (i.e. tap and long pressed) all languages have poor success rates. According to this result we conclude that involving a process of classification and association of a visual stimulus to a gesture whose nature cannot be interpreted in terms of spatial analogy with the stimulus is a more complex type of reasoning and, as pointed out by Piaget, this capability is developed by children at a later stage.

Evaluating the phase factor brings responses to RQ2 and RQ3, dealing with the improvement over time of the communication process and the language learnability, respectively. The results reveal an interesting number of different cases. Firstly, when in-place gestures are considered, none of the three evaluated languages is effective at communicating the expected gestures despite the several recall phases considered. This confirms that training has no impact on acquiring animated or iconic semiotics when in-place gestures are considered because of the cognitive limitations discussed above. Secondly, when considering dynamic gestures that involve only one contact point (i.e. drag and rotation), the results revealed that these gestures are easily learned after the initial immediate recall phase when animated languages are used. Therefore, the subsequent recall phases do not provide additional benefits when these communication languages are used. However, visual clues to communicate one-finger gestures are not learned after several recall phases when an iconic language such as the one evaluated here is used. Finally, when dynamic gestures with two contact points are considered, the results show that the effectiveness of the communication process improved after the training process, so that, RQ2 is answered affirmatively for them. In addition, RQ3 is answered affirmatively for these gestures since the results show significant differences between phases depending on the language used. The animated languages have a higher learning potential and reached an overall $90 \%$ success rate after all the recall training phases. However, the iconic semiotic is again not learned after several training phases and only reached a $20 \%$ success rate in the final tests.

In response to RQ4 (concerning the effect of gender on the effectiveness of the communication process) the results show that overall, i.e. for all the languages, gender does not have a significant effect on the success rate. However, the results from analyzing the gender and language factors (RQ5) revealed significant differences for 
Kindertivity: Usability and Communicability Strategies for Interactive Surfaces and PreKindergarten Children

the scale down gesture in which males have a higher success rate than females. In the case of the scale up gesture, males also perform on average better than females (see Figure 60) even though these differences are not statistically significant. In other words, communicating gestures with two contact points are more effective in males than in females. This is consistent with the results in (Nacher, Jaen, \& Catala, 2014) which showed that males were more effective than females in the scale up task. These results were consistent with previous studies on pre-kindergarten children, which found that, on average, preschool boys are more accurate than girls in spatial tasks (Levine et al., 1999). To overcome this small disadvantage, designers of future multi-touch applications could exploit girls' superiority in terms of verbal and oral skills (Burton, Henninger, \& Hafetz, 2005) (Berglund, Eriksson, \& Westerlund, 2005) and, consequently, include an audio channel that provides descriptions for gestures requiring spatial reasoning.

Finally, as children begin their preoperational stage at 24 months (Piaget, 1973) and then gradually develop layers of symbolic behaviors as they are introduced to reading symbols (Gardner, 1993) we evaluate the impact of age to find out whether there are any semiotics suitable for children at their initial stage of symbolic behavior development. Hence, answering the research questions that consider the impact of age, the results show that in general there are no differences between the two age groups in the in-place and the one contact point dynamic gestures (RQ6). However, older children are better than younger ones in the two scale gestures, with significant differences in the scale down task and a higher average success rate in the scale up. When the age factor is considered jointly with the language factor (RQ7) the success rate of the in-place and the one contact point tasks evolves equally with age for the animated and the iconic languages. However, it can be seen that for gestures involving two contact points, success rates improve with age only for the animated semiotics but not for the iconic one. The iconic approach does not therefore seem an effective way of communicating these gestures. This could help designers to develop tailored interfaces to the actual skills of children taking into account their development differences. Finally, answering RQ8, which considers the interaction between the gender and age group factors, an interesting situation was revealed in which younger females were more affected than older ones in terms of success when dynamic gestures with two contact points (i.e. scale up and down) were communicated. This confirms the previous results and the results of Levine (Levine et al., 1999) with respect to spatial tasks, suggesting that males develop visual-spatial cognition abilities before females. In this regard, our results show that girls between 2 and 3 years old are in the process of developing visual-spatial cognition and that this development affects the way the communication of two-finger gestures with visual semiotics is perceived. 
The results discussed in this paper and those of previous usability studies with kindergarten children and multi-touch technologies (Nacher et al., 2015) (Vatavu et al., 2015) could trigger the design of multi-touch applications that make it easier for children to use these devices on their own without the participation of a supervising adult. This should be a serious concern because these applications could be potentially harmful. As we already discussed in the Introduction, designers of these educational applications must implement scenarios that trigger and enhance collaborative dialogic learning with the participation of educators who have full control of the learning activity. These scenarios have been proven very useful with other media (books and videos) and additional work needs to be done to study the suitability of the new multitouch devices to support and enhance dialogic activities. The languages proposed in this study would enable instructors to concentrate on the dialogs related to the learning activity and would reduce the number of times they have to interfere in the interaction mechanisms expected each time by the application. The impact of these graphical languages on the quality of the dialogs established between kindergarten children and instructors is an interesting open area that would need further research.

As designers of future multi-touch applications for kindergarten children, we have the responsibility of guaranteeing that these applications are suitable for children at this early age and that they are always used under supervision. We should carefully implement security mechanisms that prevent these very young children from using potentially harmful applications by themselves. These precautions must not only concentrate on the recommendations made by most pediatric associations (American Academy of Pediatrics, 2011) for reduced screen time for under 2-year olds, but also on the appropriate learning scenarios for each developmental phase.

\subsection{Threats to validity}

Several precautions must be taken before generalizing the results obtained in this study to other contexts.

The experiments involved only one interactive element at a time in the user interface, but in certain contexts the gestures happen consecutively and so some of the results might not apply in these cases, since there is evidence that some events are affected by previous and subsequent ones (Hinrichs \& Carpendale, 2011). Hence, we need to study the effect of space cluttering in situations in which several interactive elements with their corresponding cues are displayed simultaneously and determine whether a series of gestures would make these less successful for pre-kindergarten users.

Another point to consider is that despite the fact that the participants had no previous exposure to the semiotics under evaluation, there is still a chance that they reproduce modeled behavior imitating their parent's interactions. However, because we are evaluating six different gestures, the possibility of matching the correct gesture by 
Kindertivity: Usability and Communicability Strategies for Interactive Surfaces and PreKindergarten Children

chance is low. Finally, the generalization of the results should be taken with caution and an additional experiment with a larger sample size should be performed.

With respect to the results of the iconic language, only one language was evaluated so that there are some threats to the generalization of the findings in this particular type of semiotics. Future evaluations of other iconic approaches to communicate gestures therefore need to be explored.

\subsection{Conclusions and future work}

In this work we have evaluated three languages in order to analyze their suitability to communicate multi-touch gestures to pre-kindergarten children. On one hand, the results have shown that the two animated approaches are a good way to communicate dynamic gestures such as drag, rotation, scale up and scale down, which can be communicated with success rates of up to $90 \%$. The more complex cues used when communicating dynamic gestures with two contact points (i.e. scale up and scale down) were learned by pre-kindergarteners using animated languages when several recall sessions were applied. This evaluation showed that there are no differences between the two animated approaches, so it would not be mandatory to use the explicit representation of a hand to communicate gestures since the animation of the object itself is also an effective approach. This would be a valuable design choice for interfaces with limited display space available in which the use of an explicit animated hand could be avoided.

On the other hand, the results showed that all the languages have poor success rates for in-place interactions and the communication of these gestures seems not to be learnable after several displays of the visual cues. This occurs because this type of gesture requires a process of classification and association of a visual stimulus to a gesture, a cognitive capability developed by children at a later stage.

Additionally, the results have shown that, due to the differences in visual-spatial cognition between males and females, males perceive the communication of twocontact-point dynamic gestures in a more effective way than females. However, these differences were only found in the younger age group (infants less than 30 months of age). This means that mechanisms to communicate these gestures for very young girls should be designed and evaluated.

Given that this study considers gestures and their corresponding visual cues in isolation, an interesting line of future work would be to evaluate languages and communicability strategies when several interactive elements are shown simultaneously. In this respect, an evaluation with an actual game will be one of our next future activities. 
Our plans also include the evaluation of the most successful visual semiotics with audio feedback that describe the gesture to be performed with specially designed sentences that can be understood by pre-kindergarten children and the study of the impact of these forms of media in the dialogs established between instructors and children during collaborative learning scenarios. Another interesting issue would be an evaluation of these communication strategies to find out whether users with special needs, such as children with Down's syndrome or autism require customized versions of the semiotics considered in this study.

\section{Acknowledgments}

The authors would like to thank the Universitat Politécnica de Valéncia ${ }^{9}$ and the ItuItu ${ }^{10}$ pre-kindergarten schools for their collaboration during the development of this study. This work received financial support from the Spanish Ministry of Economy and Competitiveness; European Development Regional Fund (EDRF-FEDER, with the project TIN2014-60077-R (SUPEREMOS)). Ministerio de Educación, Cultura y Deporte (Spanish Government; a predoctoral fellowship within the FPU program to V.N., FPU14/00136); Conselleria d'Educació, Cultura i Esport (Generalitat Valenciana; a postdoctoral fellowship within the VALi+d program to A.C., APOSTD/2013/013).

\footnotetext{
9 ampa.blogs.upv.es

10

www.ituitu.es
} 
Kindertivity: Usability and Communicability Strategies for Interactive Surfaces and PreKindergarten Children

\section{Appendix}

Table 20. P-value of the post-hoc pair-wise comparison of sessions by task (Bonferroni correction applied).

\begin{tabular}{|l|r|r|r|}
\hline Sessions & $\begin{array}{l}\text { Intuitive vs } \\
\text { Short Recall }\end{array}$ & $\begin{array}{l}\text { Intuitive vs } \\
\text { Long Recall }\end{array}$ & $\begin{array}{l}\text { Short Recall vs } \\
\text { Long Recall }\end{array}$ \\
\hline Tap & .190 & .956 & 1.000 \\
\hline Long pressed & .345 & $\mathbf{. 0 1 8}$ & .934 \\
\hline Drag & .308 & 1.000 & 1.000 \\
\hline Rotation & .305 & .215 & 1.000 \\
\hline Scale up & .343 & .099 & .382 \\
\hline Scale down & $\mathbf{. 0 2 2}$ & $\mathbf{. 0 0 3}$ & .243 \\
\hline
\end{tabular}

Table 21. P-value of the multiple comparison between languages by tasks (Bonferroni correction applied).

\begin{tabular}{|l|r|lr|r|}
\hline Sessions & $\begin{array}{l}\text { Animated Hand vs } \\
\text { Animated Object }\end{array}$ & $\begin{array}{l}\text { Animated Hand vs } \\
\text { Iconic }\end{array}$ & $\begin{array}{l}\text { Animated Object vs } \\
\text { Iconic }\end{array}$ \\
\hline Tap & 1.000 & 1.000 & 1.000 \\
\hline Long pressed & .954 & .227 & $\mathbf{0 3 4}$ \\
\hline Drag & 1.000 & $<.001$ & $<.001$ \\
\hline Rotation & 1.000 & $\mathbf{. 0 0 7}$ & $\mathbf{. 0 0 2}$ \\
\hline Scale up & 1.000 & $\mathbf{< . 0 0 1}$ & $<.001$ \\
\hline Scale down & 1.000 & $\mathbf{< . 0 0 1}$ & $<.001$ \\
\hline
\end{tabular}




\section{Chapter 11}

\section{Exploring Visual}

Languages for

Communicating

Directional Awareness

to Kindergarten

Children

Vicente Nacher, Sandra Jurdi, Javier Jaen, Fernando Garcia-Sanjuan

ISSI Group, Dpto. Sistemas Informáticos y Computación, Universitat Politècnica de València.

Submitted to International Journal of Human-Computer Studies. Under review, as of October 2018. 
Kindertivity: Usability and Communicability Strategies for Interactive Surfaces and PreKindergarten Children

\begin{abstract}
Although a myriad of educational applications using tablets and multi-touch technology for kindergarten children have been developed in the last decade, most of these applications do not fully exploit multi-touch technology since the game world used is limited to the screen only. Considering a larger digital space in tablet-based educational scenarios would be beneficial since it would enable the design of engaging activities driven by curiosity, exploration, discovery and decisions on where the next action is situated in the digital virtual space by directional awareness. This paper therefore investigates kindergarten children's abilities to use a virtual world beyond the screen and evaluates three different visual languages for communicating directional awareness. The results obtained show, firstly, that these specific users are able to use the space beyond the screen boundaries and that the evaluated visual languages can effectively communicate information to kindergarten children. The paper also makes a set of recommendations to help designers choose the appropriate visual language for their application requirements.
\end{abstract}

\title{
11.1. Introduction
}

Since the appearance of touch-based devices such as tabletops and tablets, very young children have had the opportunity to use new forms of interactive media to engage in beneficial educational activities (Nacher, Garcia-Sanjuan, et al., 2016b). Moreover, as (Hourcade, 2007) stated, direct-touch is preferred by children over other mediated pointing devices like the mouse, as it provides a more direct way of selecting options on the screen. Different studies have thus focused on studying the suitability of this technology for kindergarten children. The multi-touch interaction has been reported as a more intuitive way of interaction (Smith et al., 2012) that enables users to interact with multi-touch tabletops intuitively (Jokisch et al., 2011); (Mihajlov et al., 2014); (Ioannou et al., 2013). Other studies have evaluated the suitability of smaller devices and reported that even children aged 2 to 3 are able to perform a basic set of multitouch gestures (tap, scale up, scale down and rotation) on a tablet without assistance (Nacher et al., 2015) and more complex gestures (such as double tap and long press) can be performed when some assistive techniques are used (Nacher, Jaen, Catala, et al., 2014). In the same way, Vatuavu et al (Vatavu et al., 2015) showed that children aged 3 to 6 are able to perform touch gestures in both tablets and smartphones. Children's preference for educational tablet-based games has already been demonstrated (Furió et al., 2013) because it involves a natural interaction style requiring little training (Fernández-López et al., 2013). As a result, several works (Rideout, 2011); (Lydia Plowman, Stevenson, Stephen, \& McPake, 2012); (Cristia \& Seidl, 2015) have pointed out that very young children are frequent users of multi-touch devices and are confronted with this technology even before they fully develop oral communicative functions. 
Despite this growth in the use of multi-touch technology by kindergarten children, introducing multi-touch interaction is not the only challenge to be addressed when designing learning applications for them, as discussed in (Falloon, 2013). The design process is especially challenging because kindergarteners are in the process of early language development and the younger the children are the more scaffolding of technical nature they need (Neumann, 2017), including special communication strategies when using touch screen devices. Application designers thus need adequate strategies to enable young children to interpret information about the applications, such as the gestures to be performed at a given time, the actions needed to go ahead, or information about the spatial location of objects in the virtual world. Therefore, the design of efficient and effective communication visual languages which gives the user information about the application and the expected actions that the users should make is a key challenge. The design of appropriate semiotics must be addressed since, as pointed out by Derboven (Derboven et al., 2012), multi-touch interfaces can facilitate dialogic learning scenarios in which the dialog is centered around the learning activity itself rather than on the interactions the children are expected to perform each time. Hence, designing visual languages that avoid the continuous technical scaffolding by adults (i.e. the gestures to be performed, the direction in which a game character should move, etc.) will help caretakers to concentrate on giving cognitive scaffolding (i.e. the learning content to be acquired by the children). In addition, in scenarios in which collaboration is allowed between users, the presence of an appropriate visual language understandable for both children and adults would foster a smoother experience and avoid the need to provide incessant instructions and cues. Although designing visual languages tailored to kindergarten children is not aimed at allowing them to use the applications by themselves without adult supervision, the use of appropriate languages can foster more dialogues about the learning content of the application by reducing the need for technical intervention.

Although the use of touch screen devices by kindergarten children has recently received a great deal of attention in terms of gesture usability, few studies have focused on evaluating suitable semiotics for them. For example, some recent studies (Hiniker et al., 2015) (Nacher et al., 2017) have evaluated possible visual languages for communicating which are the gestures expected by the application to kindergarten children. These works show that kindergarten children are able to understand semiotic communications when using languages specially designed for them, but not when languages targeted to adults are used. They show that it is possible to design visual languages for communicating gestures for these specific users and suggest that kindergarteners are able to understand visual cues to communicate information relative to the application. However, although these works point out the importance of designing tailored visual languages for kindergarteners, there are no studies focused on the assessment of directional awareness communication tailored to this type of user. Designing appropriate directional awareness visual languages understandable by children would have several benefits. Firstly, it would reduce the technical scaffolding 
Kindertivity: Usability and Communicability Strategies for Interactive Surfaces and PreKindergarten Children

provided by parents/caretakers when interacting with applications, enabling them to focus on the learning content rather than on the interaction mechanisms. Secondly, it would enable the creation of educational scenarios in which the digital space to be explored exceeds the physical boundaries of the screen, engaging kindergarteners in activities driven by curiosity, exploration, discovery and decision-making about where the next action will take place is in the digital virtual space. Thirdly, it would contribute to the development of this skill (spatial and directional awareness) as an important cognitive ability at this early age.

In this paper we therefore consider the problem of effectively communicating directional clues to kindergarteners and evaluate three different proposed directional awareness visual languages in order to determine pre-kindergarten users' understanding of the information that they communicate. The three languages under consideration in this work are of a graphical nature because, even though it has already been shown that including instructions in the form of a short text or video clip is suitable for primary school children (Kähkönen \& Ovaska, 2006); (Niemi \& Ovaska, 2007); (Van Der Meij \& Van Der Meij, 2014), pre-kindergarten users do not have the abilities required to read and understand text messages or complex verbal video instructions.

The contributions of this work are manifold: the first is a review of 100 commercial applications of multi-touch devices targeted to kindergarten children, revealing that most of these applications only use the limited screen size as the actual interaction space in which the educational activity takes place. The second contribution is the experimental confirmation that kindergarteners are able to use the space beyond the screen limits as a virtual world. The third is the experimental confirmation that visual languages can be used to effectively communicate spatial directional information to kindergarten children. The fourth contribution is a set of recommendations guiding designers when choosing the language which best fits with the application requirements in terms of time, visual interference and relative positioning awareness.

\subsection{Related work}

Several studies have evaluated the suitability of multi-touch technology with prekindergarten children. However, as has previously been pointed out, there is no standard way of communicating information to children. In order to reduce the number of instructions given by caretakers or parents, several works have evaluated different ways of providing children with instructions about the required interaction. For example, Niemi \& Ovaska (Niemi \& Ovaska 2007), explored an interaction design process with 6 year-old children when instructions are given. Their results show that instructions in the form of animations to show the correct use of complex tools were best understood by children only if spoken instructions were also provided. Obviously, this result could have been expected, because applications for children cannot rely on written text to give instructions due to the lack of reading skills. Another example is 
the work by Kähkönen et al (Kähkönen \& Ovaska, 2006) who explored different ways of providing instructions in applications targeted at 5-6 year-old children with desktop computers. Written instructions and animations were tested and the results showed that despite communicability being especially challenging with young children, following some design guidelines was effective in supporting the communication process. They recommended giving visual cues to trigger children's attention to help them find new content and textual instructions adapted for children and providing video instruction separated from the other modalities so that they can focus on a specific explanation. The results also showed that providing help in the form of audio messages could overcome some of the limitations of written instructions.

McKnight and Fitton (McKnight \& Fitton 2010) performed a test on common touchscreen terminology in which English-speaking children aged between 6 and 7 were asked to perform a basic set of touch gestures from audio and written instructions. Their results showed that these young users had little or no trouble in understanding most of the instructions and completed the task easily. Giving instructions in both textual and audio form was thus seen to be useful, and even less familiar terms such as "slide" or "swipe" were well understood. However, they concluded that due to the children's different abilities it was hard to establish a consistent link between a term and a touch gesture, which makes giving instructions particularly critical in systems designed for them due to their limited vocabulary and reading skills.

Another approach to communicating multi-touch gestures to 5-6-year-olds was explored by Baloian et al. (Baloian et al., 2013). The study used words and prerecorded audio sources to ask users to identify and perform different gestures. Instead of referring to gestures as adults would (e.g. by their name), the researchers used metaphors for each of the application's gestures. They associated each gesture to a specific "recallable" character (e.g. a jumping grasshopper for a double tap, a walking ladybug for a drag gesture or a hovering butterfly for a tilting gesture). However, the results showed no strong correlations between performance and the behavior of the characters that the children liked or disliked the most.

The cited works considered giving instructions with a combination of text, audio and/or visual cues with children aged 5 to 7 , showing that they are ready to use this type of communication despite its having some minor issues. However, applications for very young children cannot rely on written text to give information due to the lack of reading skills and there is no standardized way to name touch gestures, so using audio cues may present several issues and may cause interferences in the parents/caretakerschildren dialogues.

Hiniker et al (Hiniker et al., 2015) evaluated prompts such as in-app audio, on-screen demonstrations (with hand demos or changing the visual state of the item) and instructions by an adult model for eliciting gestures such as double tap, horizontal and vertical swipe and shaking the tablet with children aged between 2 and 5 . Their results showed that although the most effective technique was adult guidance, children aged 3 
Kindertivity: Usability and Communicability Strategies for Interactive Surfaces and PreKindergarten Children

years or older were able to follow other types of cues. Nacher et al (Nacher, Jaen, \& Catala, 2014) (Nacher et al., 2017) analyzed the communicability of three types of touch gestures (in-place, one-contact dynamic \& two-contact points dynamic gestures) comparing three visual languages with children aged 2 to 3 . Their results showed that although the iconic approach designed for adults is not appropriate for young children, the two animated languages evaluated had high success rates (reaching 90\%) when communicating gestures which involve movement (drag, rotation \& scales). Hence, the basic reasoning related to the interpretation of moving elements on a surface can be effectively performed during early childhood. This is an interesting result because developmental psychologists such as Levine and Piaget suggest that kids develop spatial reasoning during middle childhood (Levine et al., 1999) (Piaget, 1973). However, the above-cited study suggests that basic reasoning related to the interpretation of moving elements on a surface can be effectively performed during early childhood.

These latest works focus on evaluating semiotics for giving instructions which use only visual cues with children aged from 3 to 5 . Although their results showed that these approaches are feasible and understandable for these users, the studies focus on semiotics for giving instructions to children but not for giving any type of application information, such as directional awareness, spatial information, application goals, etc. As Markopoulos and Bekker (Markopoulos \& Bekker, 2003) point out, interfaces for children should be designed according to their development stages and their actual needs.

Despite the lack of works that consider communication strategies for kindergarterners with respect to spatial directional awareness in the context of computer-based learning applications, there have been a number of works that propose strategies for teachers to help children develop spatial literacy. This is the case of Golbeck (Golbeck, 2005), who proposes ways of promoting spatial literacy in the study of mathematics, social studies, science, literacy, and visual arts. Interestingly, the author affirms that "with experience and growing memory capabilities, children shift from a uni-axial to a biaxial system for spatial representation". This would encourage the use of current interactive technologies to help children make this shift by exposing them to experiences in which spatial awareness is exercised. It is also interesting to point out here that many works (Case, Griffin, \& Kelly, 2001; Case, Stephenson, Bleiker, \& Okamoto, 2008; Demetriou, Christou, Spanoudis, \& Platsidou, 2002) have studied how children develop spatial thinking by analyzing how they produce drawings and how space is represented in them, e.g., from depicting objects floating in space weightlessly without a reference coordinate system in their pre-axial phase to fully integrating two or more reference lines and considering perspective and depth relationships by age 10 . However, these studies just consider what spatial information children are able to produce in terms of drawings but not whether they are capable of decoding, understanding and using spatial knowledge that is provided to them by some sort of graphical language. These studies, which conclude that children are not able to 
consistently create bi-axial representations until the age of 8 have probably made designers of learning applications for kindergarteners think that at this early age children are not able to interpret and use bi-axial spatial or directional information.

The present study will therefore evaluate whether this assumption is sound or whether there is space for improvement by designing applications that help children to explore bi-axial interactive spaces whose limits are outside the reduced screen size of current multi-touch devices.

\subsubsection{Industrial perspective on the communicability of spatial and directional awareness in touch devices}

Besides the review of the studies from a research perspective in the previous section, it is important to examine how commercial applications are addressing the communicability of spatial and directional information about their digital world. With this purpose, 100 educational applications ${ }^{11}$ were randomly selected from the collection of the kindergarten educational applications in the Android App Store and were analyzed in terms of usage of a space beyond what is being displayed on screen (i.e. whether the application camera is fixed and always displays the whole space or can be moved to reach other parts not displayed at a given time). When there was a digital world beyond the boundaries of the screen, we analyzed the mechanisms used to provide directional awareness to users, if any.

The results of this analysis revealed that only $33 \%$ of the applications used a game world beyond the screen size. Most of the applications targeted to kindergarten children are limited to simply using the screen size as the digital world that is always shown completely. Only $5 \%$ of the reviewed applications (15.15\% of the applications that use the world beyond the screen) use any type of mechanism to provide directional awareness to the users.

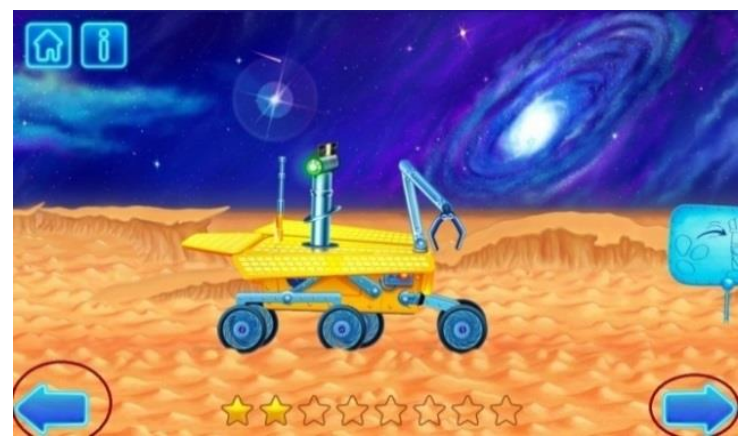

Figure 64. Example of communication used in a commercial application.

\footnotetext{
$11_{\text {goo.gl/vG } 88 f M}$
} 
Kindertivity: Usability and Communicability Strategies for Interactive Surfaces and PreKindergarten Children

Two different techniques were identified: the first (used in three applications) is an arrow to signal the direction that the character has to follow to proceed in the task/game (see Figure 64).

The other technique (used in two applications) is a dynamic semiotic that indicates the direction in which the target objects (i.e. the objects to be reached by the main character of the game) can be found when they are not being displayed on the screen. To do that, when an object "disappears" because its position is outside of the screen limits, a miniature of this object is shown in the last visible position and a dynamic arrow moving around it indicates the direction in which the object can be found (see Figure 65).

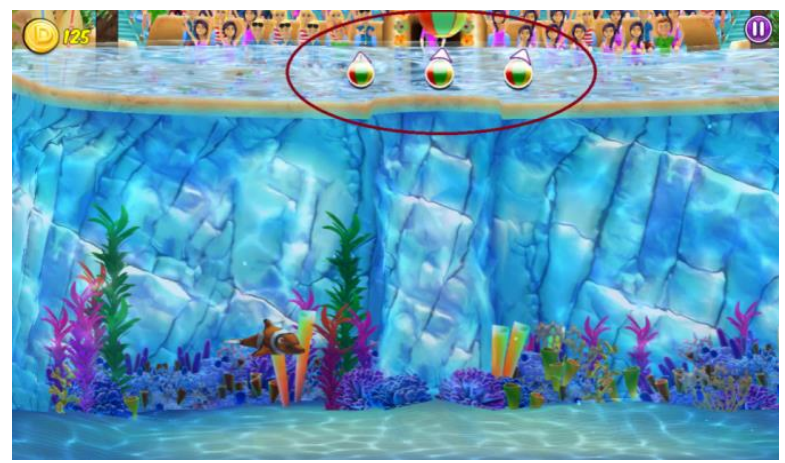

Figure 65. Example of communication used in a commercial application.

The main conclusion that can be drawn from these results is that, even though there is a popular commercial trend in developing apps for kindergarten children with touchscreen technology (Nacher et al., 2015), developers in general simply use the screen size as the boundary of the game/application world. Few applications use some sort of visual language to provide directional or spatial awareness to users and there is no standard and validated way of providing this information. In this work we therefore evaluate whether kindergarten children are able to interact/play with applications in which the digital world is not limited to the screen only and then evaluate three different visual languages to provide spatial directional feedback to children when interacting with an application of this type. The empirical evaluation and the results obtained in this work are a step forward in the process of obtaining an effective language to communicate directional information to kindergarten children that could be used in educational applications based on this technology.

\subsection{Visual languages for directional awareness}

In this work, we use visual languages to provide kindergarten children with directional awareness of the digital world beyond the area being displayed on the screen at a specific time. This directional awareness is the information about the direction that the 
Exploring visual languages for communicating directional awareness to kindergarten children

main character in the game should follow to reach the different objects that need to be picked up or visited in order to succeed in the game task. In this context, the selected game style is that of adventure games in which children have to control the movements of a character to explore the surrounding space in search of items. The choice of this type of game style is because it stimulates curiosity (Collins \& Stevens, 1981) (Malone, 1981) and can potentially facilitate a range of different learning styles such as tutoring, practice and self-learning (Dempsey, Rasmussen, \& Lucassen, 1994). In addition, this type of games fosters learning discovery which is a technique that helps learners to create and organize their knowledge, since they draw upon past knowledge and experience to infer underlying strategies and gain understanding of concepts (Honomichl \& Chen, 2012). Knowledge discovery is also beneficial for students' motivation, since those who discover information for themselves are more motivated to achieve educational goals and more likely to remember the information learned (Bruner, 1960). Hence, the proposed directional awareness languages need to give information about where the main character of the game is with respect to the target object in order to help children to effectively guide the character towards the correct destination. Previous works regarding the use of visual languages to communicate information to children (Downs, Liben, \& Daggs, 1988) (Thomas, Nye, \& Robinson, 1994) (Leekam, Perner, Healey, \& Sewell, 2008) reveal that to understand symbols, children require an understanding of the object-referent relation and the informational value of the sign along with its substitutional function. If the child can neither detect the information a sign conveys nor use it as a representation of its referent, a lack of communication is the consequence. As pointed out by (Catling, 2005), it is true that the ability to derive proper meaning from symbols on maps is developmentally related and gradually improved, (Goria \& Papadopoulou, 2012) maintain that preschoolers use iconicity to a large extent instead of symbols to represent spatial cartographic information. This would suggest that, in theory, an iconic style would be the best approach to communicate directional awareness. However, there are no studies that confirm this superiority with respect to a symbolic choice when communicating directional awareness. When communicating this awareness, icons need to be placed within a cartographic context to convey direction and this issue has not been previously studied in preschoolers. In this study we therefore selected three visual styles to study their effectiveness with respect to directional awareness: a pure symbolic language, an iconic with local cartography language and an iconic with global cartography language. In order to select suitable candidate languages for evaluation, several workshops took place with kindergarten educators and pedagogical experts. As a result, the following mechanisms were selected for evaluation with these children:

- Mini-map (icons over global cartography): in this case, a miniature map is placed at the bottom right corner of the screen (see Figure 66). This map contains the entire digital world displaying all the existing objects and the main character. In this case, the destination element is marked in the mini-map with a red stripe around it (see Figure 66).Using this technique, the users are aware of the position of all the objects in the 
Kindertivity: Usability and Communicability Strategies for Interactive Surfaces and PreKindergarten Children

game and the distance to reach them. This type of language is often used in games targeted at adult players.

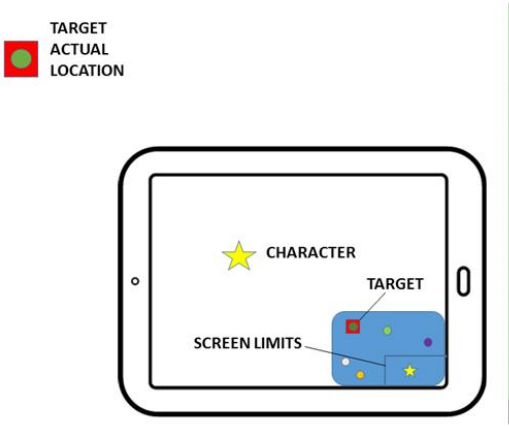

(a)

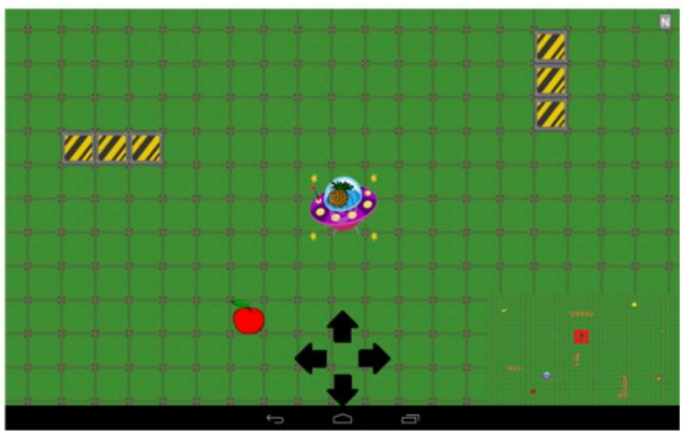

(b)

Figure 66. Example of the task with the mini-map (a) abstraction (b) application snapshot.

- Border-Floating thumbnails (icons over local cartography): in this technique, miniatures of the objects that are not visible on the surface appear at the border of the screen. The position where the miniature is shown is the intersection between the vector that links the character to the corresponding object and the screen border (see Figure 67). The miniature positions are dynamically updated according to the relative character's position at a given time. With this technique, the language only represents the objects that are outside the current screen display.

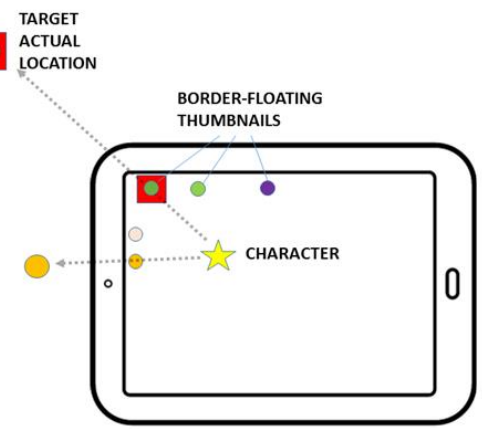

(a)

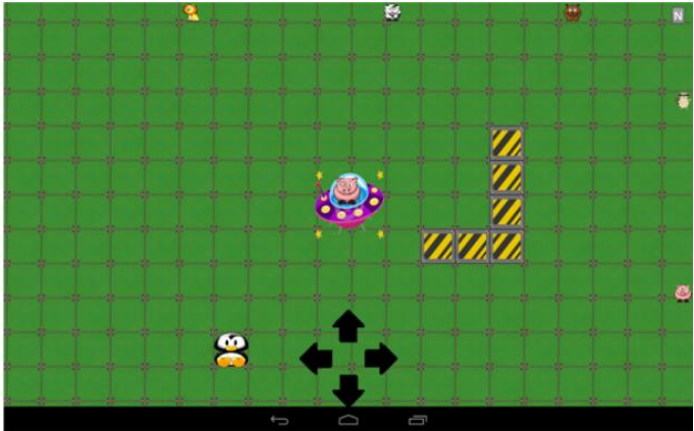

(b)

Figure 67. Example of the task with the thumbnail language (a) abstraction (b) application snapshot.

- Arrow (symbolic): in this case, the technique uses an arrow to indicate the direction to follow to reach the current target (see Figure 68). The arrow is dynamically updated and will move around the character according to the trajectory to be followed to reach 
the destination. In this technique, information on the relative positions of other objects is not represented.

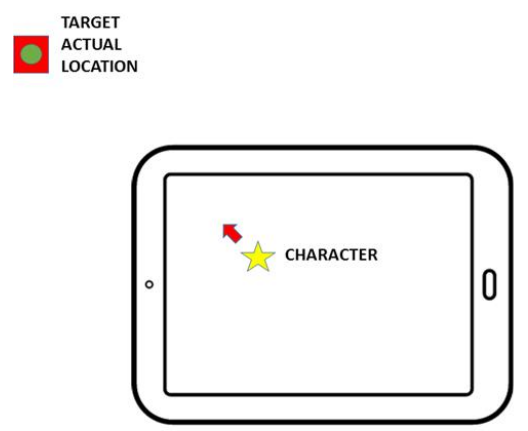

(a)

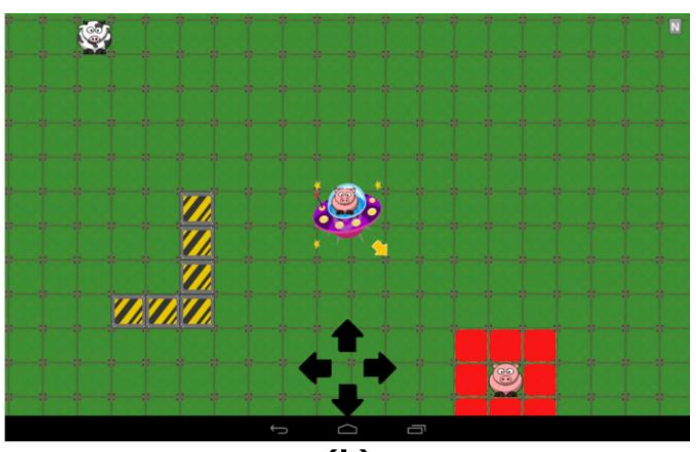

(b)

Figure 68. Example of the task with the arrow language (a) abstraction (b) application snapshot.

\subsection{Study context}

The overall goal of our study was to test the suitability of the previous visual languages for providing directional awareness of the objects in a digital 2D game world to kindergarten children and to evaluate their effectiveness and efficiency. One of the mechanisms, the mini-map, is often used with adult users and the other two were designed for children. Hence, using the GQM (Goal Question Metric) template (Basili et al., 1994), our goal can be defined as follows: analyze three visual languages for the purpose of evaluating their suitability from the viewpoint of effectiveness and efficiency in the context of providing directional awareness of the objects in a digital game world to kindergarten children.

For this study we considered children of both genders aged between 4 and 7 . According to developmental theories, children are continuously developing and refining their cognitive skills and are in the preoperational stage of development until the age of 7 (Piaget, 1973). As we were interested in exploring how the proposed visual languages are performed by young children and how the development of their cognitive skills affects their proficiency, we defined three age groups; children aged between 4-5, 5-6 and 6-7.

Consequently, the research questions of this work may be formulated as follows. The first research question is about the appropriateness of using visual languages to communicate directional awareness:

- RQ1: Is any of the considered visual languages effective in providing directional awareness to kindergarten children? 
Kindertivity: Usability and Communicability Strategies for Interactive Surfaces and PreKindergarten Children

- Then, four research questions are stated and will be answered for each factor $\mathrm{F}_{\mathrm{i}}$ considered (where $i=$ Type of visual language, Age and Gender)

- RQ2: Is the effectiveness in the task affected by the factor $F_{i}$ ?

- RQ3: Is the efficiency of the task affected by the factor $F_{i}$ ?

- RQ4: Is the relative positioning awareness in the task affected by the factor $\mathrm{F}_{\mathrm{i}}$ ?

- RQ5: Is the level of visual interference with the task affected by the factor $F_{i}$ ?

\subsubsection{Participants}

Sixty children aged from 4 to 7 years old took part in the experiment (Mean $(M)=67.4$ (months), Standard Deviation $(\mathrm{SD})=9.75$ ) with a gender distribution of 26 males and 34 females.

The children were split up into three balanced age groups, i.e. they were grouped by age, with each age group a comprising the ages in $[a+1[$. The distribution of the age groups is shown in Table 22.

Table 22. Number of participants by age group

\begin{tabular}{|l|l|}
\hline Age group & \#Participants \\
\hline 4 & 20 \\
\hline 5 & 20 \\
\hline 6 & 20 \\
\hline
\end{tabular}

The 4 to 7 year age range was chosen in order to explore how the proposed visual languages are understood and learned by children in the earliest stage of development. The youngest users were children aged 4 years because in previous studies (Nacher, Ferreira, et al., 2016), children were found not to be able to move/guide a character in a 2D world using indirect drag techniques with acceptable success until 4 or older. Parental authorization was obtained before carrying out the study.

\subsubsection{Equipment}

The interaction framework for the experiment was implemented in Java using the LibGDX framework. The devices used for the experiment were BQ Edison 3 tablets with Android 4.4. The tablets were equipped with capacitive multi-touch screens.

\subsubsection{Task}

We wanted to design a task to test several factors and to ensure that all the users have the same conditions in order to compare the results. The task would allow the suitability of using the virtual space "beyond the screen" limits to be assessed with kindergarteners, evaluating whether they are able to reach several targets in a virtual 2D world and determine the suitability of the designed visual languages to facilitate dialogic learning scenarios in which the dialog is centered on the learning activity itself rather than on the interactions the children are expected to perform each time. This task was chosen because it is an initial step in designing game scenarios in which discovery 
and curiosity can be fully fostered to support storytelling activities for a bi-dimensional virtual space, adventure games in an open space, etc.

Therefore, the task consists of a game in which a spacecraft (see Figure 70) has to travel within a digital 2D space in order to pick up, in a predefined order, three of the six objects scattered around the digital world (see Figure 69 for examples (two examples by topic) of the objects scattered in each topic), which includes some blocks that cannot be traversed, to force children to plan trajectories that avoid crashing into them.

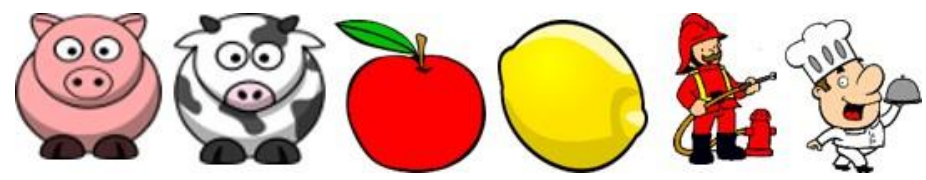

Figure 69. Example of some objects of the three diffrent topics to pick up in the game (animals, fruits and jobs).

The interaction technique selected to move the spacecraft was button-based dragging. This technique consists of four arrow-shaped buttons that are used to move the target object in the four basic directions (i.e. up, down, left and right). The buttons were placed at the bottom-center of the screen (see Figure 70). With this technique, users can move the target by tapping and holding one finger on the button that symbolizes the desired direction. This mediated interaction mechanism has been evaluated in previous studies (Nacher, Ferreira, et al., 2016) and shown to be suitable for children aged 4 years and older. This study also revealed that it was the best indirect drag technique of all those evaluated if the main priority is to avoid undesired collisions with objects in the game.

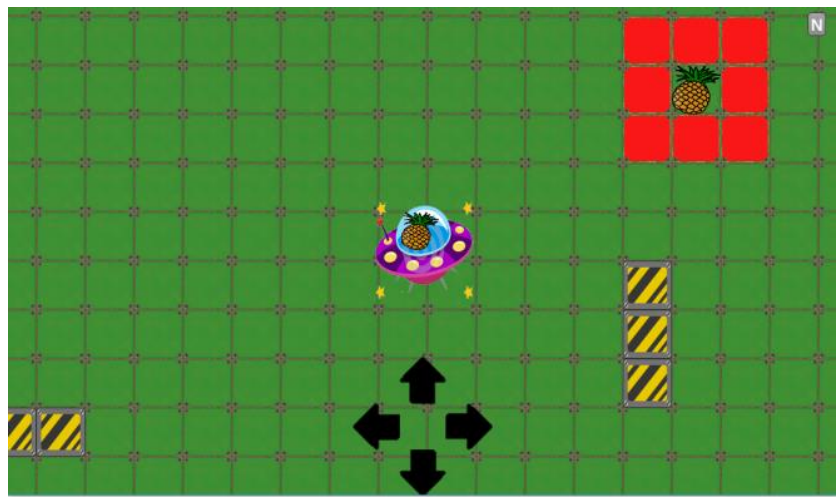

Figure 70. Task example without visual language. Topic: Fruits.

Using the button-based dragging technique, children have to guide the spacecraft through the digital world in order to pick up the three proposed objects. The current 
Kindertivity: Usability and Communicability Strategies for Interactive Surfaces and PreKindergarten Children

target to be picked up at a given time appears on the spacecraft (see Figure 70) and the cells around it are colored in red. The children then have to use the corresponding visual language to obtain directional awareness and reach the target. When the correct object has been picked, the next one appears on the spacecraft and when the three objects have been picked up the task is over.

There were three topics for the task: animals, fruits and jobs. In each topic, the pickup order is predefined and the same for all children in order to compare the results.

\subsubsection{Procedure}

The experiment was carried out on three consecutive days (one technique each day). The children performed three repetitions (one per topic) of the task daily, using one of the visual languages described above. The order in which the topics were presented and the order in which the languages were evaluated each day were randomized per subject to avoid learning effects.

At the beginning of each session, the children participate in a 5-minute learning phase with an instructor in which children get acquainted with the task and the interaction technique using the buttons-based drag. In this learning phase the instructor teaches the children that there are several objects scattered around the game world that they have to pick up in a specific order. The children are told that the spacecraft is the main character to be controlled and it displays the current object to be picked up. After these introductory explanations, the instructor makes sure that none of the children fails to identify themselves with the spacecraft. During the training session there is no directional awareness language displayed. When the test begins the children have to perform three repetitions of the task (one per topic) with the assigned visual language and there is no additional external adult intervention. When a correct object is picked up, the platform gives a positive audiovisual feedback. In the same way, if the object picked up is not the correct one a negative audiovisual feedback is given by the platform. If the instructor observes that the participant is not able to find or pick up an object in a given time, it is marked as undone and the child continues with the next item. For each task, the system records the following information: the completion time to pick up the objects; the success rate (whether they pick up the correct object in a given trial); any collisions with the blocks on the surface, the number of incorrect pickups and the distance in pixels travelled by the spacecraft across the game world in order to compare it with the optimal path for the task. A qualitative analysis is also carried out from the notes taken by an external observer during the experimental sessions.

To sum up, the children participate in the experiment for three days and each day they perform three repetitions of the task (one per topic) to pick up three of the six scattered objects in each topic in the game using a different visual language each day. 


\subsubsection{Design}

Six dependent variables were defined: success rate, completion time, travelled path, changes of direction, collisions with blocks and incorrect pickups. A mixed design was used as all the participants tested the three directional awareness visual languages. A repeated measures ANOVA (with an $\alpha=0.05$ ) was carried out with the within-subject factor Visual language with three levels (Minimap vs. Thumbnails vs. Arrow) and the between-subject factors age group (4 years vs. 5 years vs. 6 years) and gender (Male vs. Female). A Mauchly's Test of Sphericity (Mauchly, 1940) was carried out in order to determine whether the data met the sphericity assumptions. The tasks that did not comply with these assumptions were applied a Greenhouse-Geisser (Abdi, 2010) correction and were reported in the results section with F-values with fractional degrees of freedom.

\subsection{Results}

\subsubsection{Success rate}

In order to aggregate the success variable over the three repetitions of the task with each language, the variable was expressed as a percentage according to the number of repetitions performed successfully. The success rate of each visual language is given in Table 23 and shown graphically in Figure 71.

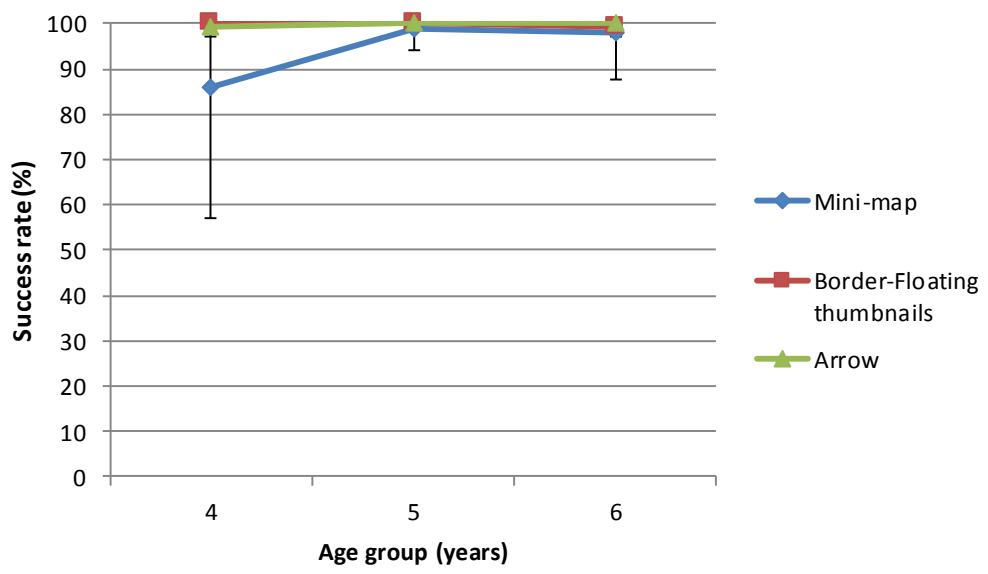

Figure 71. Success rate by visual language and age group.

The repeated measures ANOVA revealed significant differences in the within-subject factor visual language $[\mathrm{F}(1.019,58.074)=5.621, \mathrm{p}=.021]$ and in the between-subject factor Age group $[\mathrm{F}(2,57)=3.298, \mathrm{p}=.044]$ but not in the between-subject factor Gender $[\mathrm{F}(1,58)=.261, \mathrm{p}=.611]$. The post-hoc tests (see Table 29) revealed significant differences between the mini-map approach and the other two techniques. 
Kindertivity: Usability and Communicability Strategies for Interactive Surfaces and PreKindergarten Children

As can be seen in the figure, the difference in the success rate comes from the younger age group having a lower success rate. Moreover, the ANOVA revealed that there are no significant main effects visual language*age group interaction $[\mathrm{F}(2.038,58.074)=$ $2.979, \mathrm{p}=.058]$. Hence, success rate evolves with age in the same way in the three visual languages.

\subsubsection{Completion time}

With the purpose of evaluating the completion time spent by each user to perform the task, the average of each subject's successful task was used. The unsuccessful tests were excluded in the completion time analysis. Completion time can be seen in Table 24 and Figure 72 by visual language and age group.

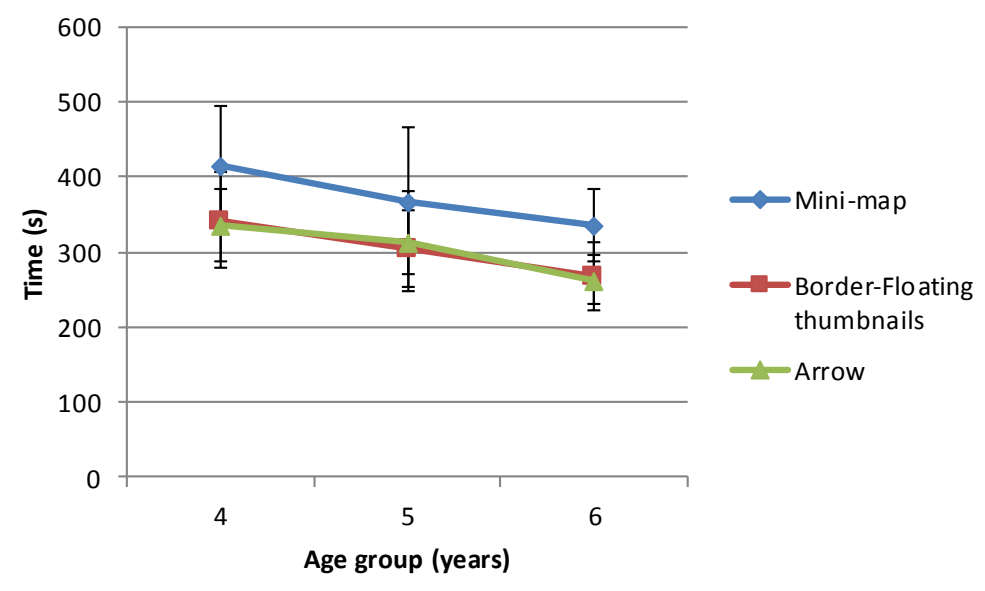

Figure 72. Completion time by visual language and age group.

The repeated measures ANOVA revealed significant differences in the within-subject factor visual language $[\mathrm{F}(2,98)=36.927, \mathrm{p}<.001]$ and in the between-subject factor Age group $[\mathrm{F}(2,49)=9.954, \mathrm{p}<.001]$ but not in the between-subject factor Gender $[\mathrm{F}(1,50)=.808, \mathrm{p}=.373]$. The conducted post-hoc tests (see Table 29) revealed that there are differences in the completion time between the mini-map language and the other two techniques, the mini-map being significantly slower $(22.4 \%$ more time needed to complete the task) than the others. The post-hoc tests (see Table 29) conducted on the age group factor revealed that there are differences between all the age groups in terms of completion time; the older they are the faster they perform the task.

The ANOVA also revealed that the completion time evolves with age in the same way for the three languages, since there are no significant effects with the visual language $*$ age group interaction $[\mathrm{F}(4,98)=.373, \mathrm{p}=.827]$. 


\subsubsection{Relative positioning awareness}

When providing location awareness, a key issue is the ability of the visual language system to effectively communicate the relative position of a target destination with respect to the current position of the user. In order to assess the effectiveness of the languages under evaluation we measured the optimality of the traversed path to reach a given destination and the number of changes of direction required to reach the target. These are two indirect metrics that provide a quantitative measure of the cognitive effort that pre-kindergarteners have to make to understand where the final destination is with respect to their current position.

\subsubsection{Optimality of traversed path}

In order to evaluate the optimality of the traversed path by the subjects with the main game character, the distance in pixels that the main character travels in each test was gathered $\left(\mathrm{d}_{\text {travelled }}\right)$. This distance was compared to the distance that would have been travelled had the path been optimal $\left(\mathrm{d}_{\text {optimal }}\right)$, i.e. the shortest path to pick up all the targets, and the absolute error was calculated. This error is expressed as a percentage over the total path distance $\left(100 \cdot\left|\mathrm{d}_{\text {travelled }}-\mathrm{d}_{\text {optimal }}\right| / \mathrm{d}_{\text {optimal }}\right)$ by visual language and age in Table 25 and Figure 73.

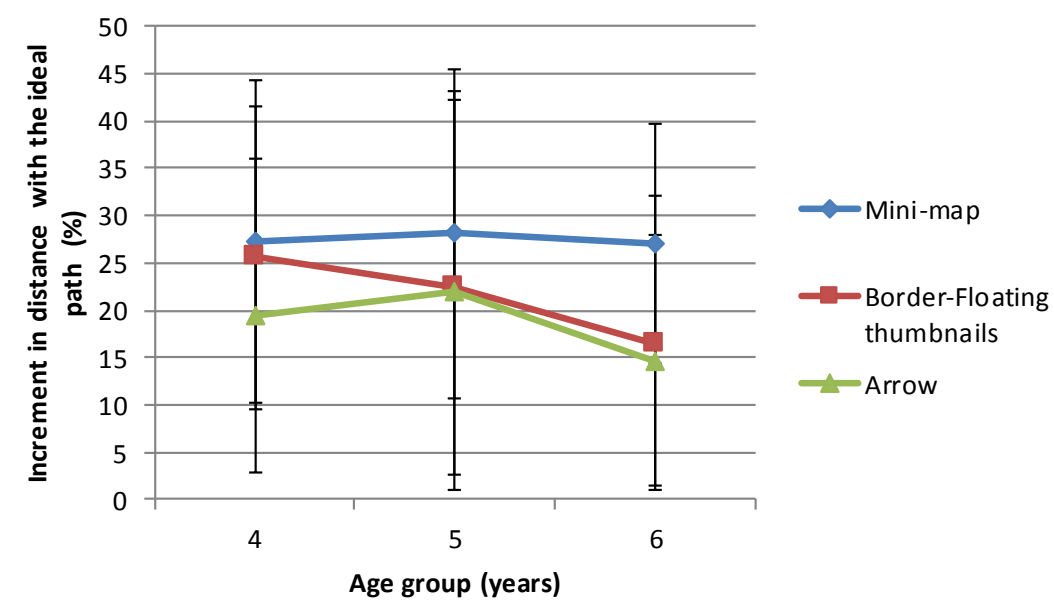

Figure 73. Increment of distance travelled by visual language and age group.

The repeated measures ANOVA revealed significant differences in the within-subject factor visual language $[\mathrm{F}(2,106)=5.700, \mathrm{p}=.004]$. The conducted post-hoc tests (see Table 29) revealed that there are differences in the increment of the travelled path between the mini-map and the arrow technique because users travelled a significant longer path when using the mini-map, $\left(\mathrm{M}_{\text {mini-map }}=27.48\right.$ and $\left.\mathrm{M}_{\text {arrow }}=18.71\right)$. No other significant differences were revealed by the post-hoc tests. The analysis did not reveal 
Kindertivity: Usability and Communicability Strategies for Interactive Surfaces and PreKindergarten Children

any significant differences in the between-subject factor Age group $[\mathrm{F}(2,53)=1.187, \mathrm{p}$ $=.313]$ and the between-subject factor Gender $[\mathrm{F}(1,54)=.610, \mathrm{p}=.438]$. Hence, the age and gender did not have a significant impact in the increment of distance in the travelled path.

The ANOVA also revealed that the increment in the travelled path evolves with age in the same way for the three languages, since there are no significant effects with the visual language $*$ age group interaction $[\mathrm{F}(4,106)=.602, \mathrm{p}=.662]$.

\subsubsection{Changes of direction}

Another way of evaluating the relative position awareness during the task is to measure the number of times each child changed the direction of the movement of the main character. These changes of movement direction reveal that the user is changing his (her) mind about the path to be followed and indirectly measure the effectiveness of the visual language to communicate the correct direction to be followed. The number of times that children changed the direction in a task is given in Table 26 and shown graphically in Figure 74 by visual language and age group.

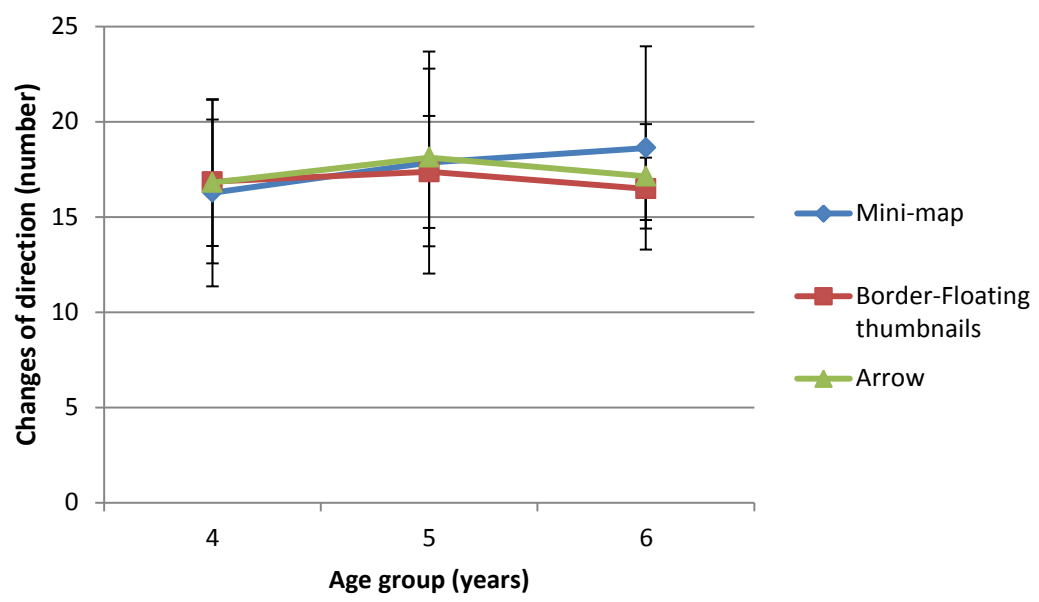

Figure 74. Changes of direction by age group and visual language.

The repeated measures ANOVA did not reveal significant differences in the withinsubject factor visual language $[\mathrm{F}(2,114)=.496, \mathrm{p}=.610]$ or in the between-subject factors Age group $[\mathrm{F}(2,57)=.865, \mathrm{p}=.427]$ and Gender $[\mathrm{F}(1,58)=.000, \mathrm{p}=.987]$.

It also revealed that the number of changes of direction evolves with age in the same way for the three techniques since there are no significant effects with the visual language ${ }^{*}$ age group interaction $[\mathrm{F}(4,114)=.756, \mathrm{p}=.556]$. 


\subsubsection{Visual Interference}

\subsubsection{Collisions with blocks}

Data was gathered on collisions with the blocks in order to evaluate the degree of interference of the visual language with the task at hand by measuring the ability of children to avoid obstacles when guiding a character in a $2 \mathrm{D}$ world. The number of collisions of the character in a task can be seen in Table 25 and Figure 75 by visual language and age group.

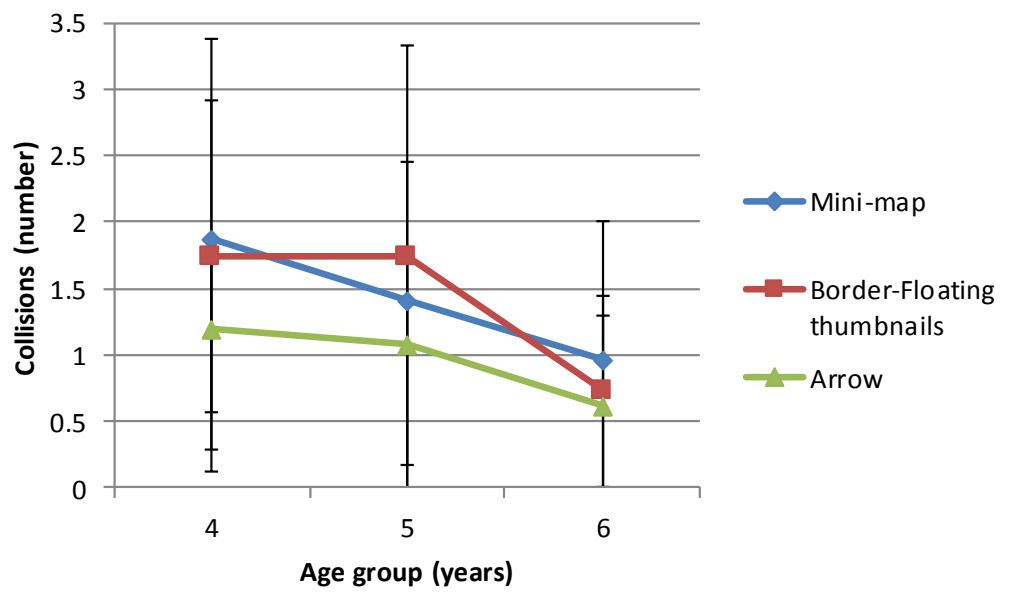

Figure 75. Number of collisions by visual language and age group.

The repeated measures ANOVA revealed significant differences in the within-subject factor visual language $[\mathrm{F}(2,114)=14.600, \mathrm{p}<.001]$ and in the between-subject factor Age group $[\mathrm{F}(2,57)=4.174, \mathrm{p}=.020]$ but not in the between-subject factor Gender $[\mathrm{F}(1,58)=.245, \mathrm{p}=.623]$.The conducted post-hoc tests (see Table 29) revealed that there are significant differences in the number of collisions with blocks between all the visual languages being the mini-map technique in which children had a higher number of collisions, followed by the border-floating thumbnails and, finally, the best technique to avoid collisions with blocks according to the results was the arrow. In addition, although there is a trend to reduce the number of collisions with age (Figure 75 ) the conducted post-hoc tests (see Table 29 ) with the age group factor only revealed significant differences between the 4-year-old and 6-year-old children, the older ones having the lowest number of collisions.

The analysis also revealed that the number of collisions evolves in the same way with age for the three languages, since there are no significant effects with the visual language ${ }^{*}$ age group interaction $[\mathrm{F}(4,114)=.935, \mathrm{p}=.447]$. 
Kindertivity: Usability and Communicability Strategies for Interactive Surfaces and PreKindergarten Children

\subsubsection{Incorrect pickups}

In order to evaluate the effectiveness of each language to locate a target without creating confusion with other objects in the game world, the number of times that children picked up the wrong object was counted. The data on incorrect pickups can be seen in Table 28 and in Figure 76 by visual language and age group.

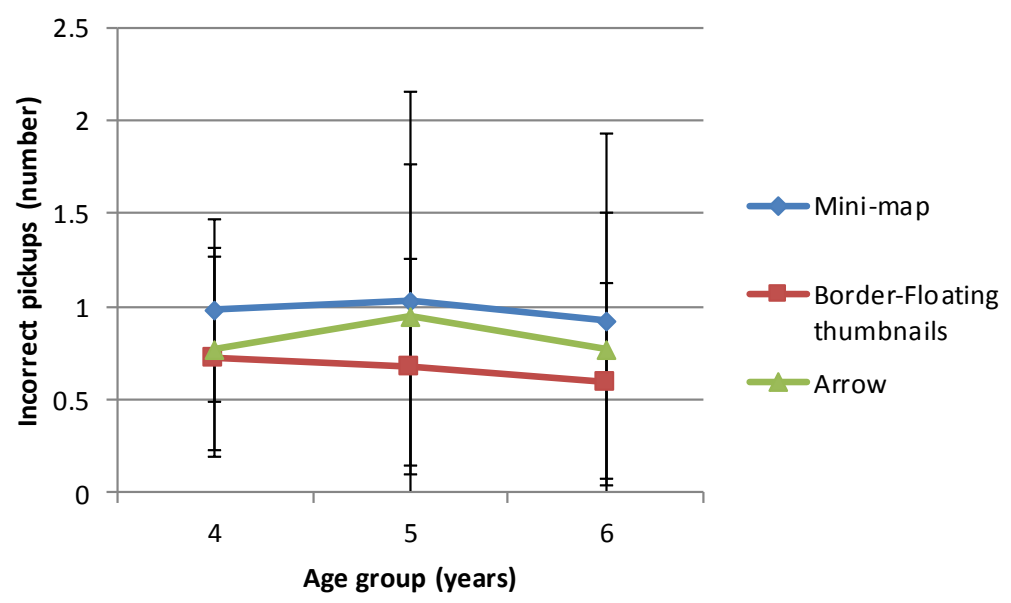

Figure 76. Number of incorrect pickups by visual language and age group.

The repeated measures ANOVA did not reveal significant differences in the withinsubject factor visual language $[\mathrm{F}(1.750,99.774)=2.907, \mathrm{p}=.066]$ or in the betweensubject factors Age group $[\mathrm{F}(2,57)=.367, \mathrm{p}=.694]$ and $\operatorname{Gender}[\mathrm{F}(1,58)=.833, \mathrm{p}=$ .365].

The ANOVA also revealed that there are no significant differences in the evolution of the number of incorrect pickups with age for the three languages, since there are no significant effects with the visual language*age group interaction $[\mathrm{F}(3.501,99.774)=$ $.144, \mathrm{p}=.952]$.

\subsection{Discussion}

The experiment explored and answered the comprehensive set of research questions that had been posed. The answer to RQ1 about whether there can be any feasible visual language to effectively provide directional awareness to kindergarten children is affirmative as the results reveal that children achieved success rates of over $90 \%$ in the evaluated languages, showing that the three evaluated directional awareness visual languages can be understood by kindergarten children.

RQ2, on whether the effectiveness of the task is affected by the type of language, is answered affirmatively since the results show that significant differences were found 
between the three evaluated visual languages. Despite having a success rate of $94 \%$, the mini-map technique was shown to cause children the most problems, whereas the border-floating thumbnails and the arrow techniques reached success rates of over $99 \%$. These results are interesting because they suggest that even very young children are able to perform the required mapping to interpret the data given by visual languages and extrapolate it to locate different objects in a virtual world. The RQ2 for the Age factor (whether the effectiveness of the task is affected by age) is affirmatively answered, revealing that the youngest age group achieved a lower success rate than the older. As can be seen in Table 23, the above-mentioned issues with the mini-map happened exclusively with the youngest age group. As Piaget (Piaget, 1973) points out, children begin their preoperational stage at 24 months and then gradually develop layers of symbolic behaviors as they are introduced to reading symbols (Gardner, 1993), so the youngest children may have some issues when understanding the information communicated by the mini-map. This should be taken into account by designers when developing applications, because the use of the mini-map technique with children aged 4 years or younger may have an impact on the effectiveness of the communication process.

RQ3, on whether the type of visual language has an impact on the efficiency of children in the task, is positively answered in terms of the time needed to complete the task, since the results showed that the mini-map technique was the slowest, followed by the border-floating thumbnails and finally the arrow technique was the fastest. Children have more difficulties and need more time to succeed in the task when the communication is given with the mini-map technique because mapping between what is being displayed on the mini-map and the virtual world is challenging for them and requires a mental context switch between both spaces. The next technique in time needed to succeed in the task is the border-floating thumbnails; in this case children have to understand that the thumbnail is being displayed on the intersection between the vector that links the main character to the target and the screen border and this mental demand may have an impact on the time. Finally, the arrow technique turned out to be the best in terms of completion time needed to succeed. In this case, the mental demand is lower since children "only" have to follow the direction to which the arrow points to reach the target. RQ3 is also affirmatively answered the Age factor, since the results show that there are differences between all the age groups, with the older children being faster. This is an expected result, given that children are continuously developing their motor and cognitive skills and the older they are the faster they are expected to perform the task. This should be taken into account by designers when time performance is a mandatory application requirement.

In response to RQ4, relative position awareness can be evaluated in several ways such as considering the optimality of the travelled path and the number of changes of direction. Analyzing the travelled path with respect to the visual language used, RQ4 is affirmatively answered. Although no differences in the distance of the travelled path are reported between the border-floating thumbnails and the arrow techniques, children 
Kindertivity: Usability and Communicability Strategies for Interactive Surfaces and PreKindergarten Children

travelled a significantly longer path when the mini-map was the communication technique used. As stated above, children may have some issues when making the mapping between what is being displayed on the mini-map to the virtual world and it has an impact on the travelled path, since they are not able to mentally define the appropriate path to follow at the beginning of the task. When performance in terms of the path is required in the application, designers should thus avoid using the mini-map technique. On the other hand, this research question is answered negatively for the Age factor, since age does not have an impact on the distance of the path covered.

In response to RQ4 by means of another indirect measure, the number of changes of direction, the results show that overall, i.e. for all the techniques, the type of technique used does not have an impact on the number of changes of direction (see Figure 74). The answer to this research question is also negative for the Age factor, since it does not have an effect on the number of changes of direction made by the users. Therefore, there are no differences between techniques when communicating where the target is and the direction in which the character has to move to reach it.

In terms of visual interference with the task, we analyzed whether paying attention to the visual language used resulted in children accidentally picking up incorrect objects or colliding against blocks. When we analyze collisions with incorrect objects, RQ5 is answered negatively for the Visual language factor, since it does not affect the number of errors that children made when picking up objects. Age does not have an impact on the number of incorrect pickups made by users (RQ5 is negatively answered in terms of incorrect pickups for the Age factor). According to the data shown in Table 28, it can be seen that all the techniques have an average number of incorrect pickups lower than 1 by task, and so we can conclude that the techniques evaluated are effective in identifying the target and do not create ambiguity with the other objects scattered around the virtual world.

If we consider RQ5 in terms of the number of collisions with blocks in the task, then RQ5 is answered affirmatively for the Visual language factor. This is an interesting result since it shows that the evaluated techniques require different attention levels. The arrow technique was shown to be the best to reduce the number of collisions with the blocks. This can be explained because the arrow technique is shown around the main character and children do not have to look away to receive the directional awareness. However, with the other two techniques the visual cue is displayed at the bottom-right corner (mini-map) or the limits of the screen (border-floating thumbnails) forcing children to divert their attention from the main character and causing more collisions. The results also show differences between the mini-map and the border-floating thumbnails techniques, the first one being more distracting for children than the second. With the border-floating thumbnails, the required movement of the main character will always be "following" the thumbnail while it is moving across the screen, hence reducing the level of interference that diverts children's attention. However, when the mini-map technique is used, the focus is always on the bottom- 
right corner of the screen where the mini-map is placed. Hence, in situations in which the character has to move in the opposite direction, there is a continuous gaze context switch causing the maximum level of interference with the task. When the visual language's level of interference needs to be minimum, designers should use the arrow technique. RQ5 is also affirmatively answered for the Age factor since the older the children the fewer the number of collisions. This is an expected result since the older the children are the better they manage the cognitive load caused by visual context switch.

Finally, regarding the impact of gender, the results show that in general there are no differences between males and females in any of the evaluated dependent variables, so that all the research questions (RQ2, RQ3, RQ4 and RQ5) are answered negatively for the Gender factor. This is an interesting result since it shows that even though previous studies with pre-kindergarten children have found that, on average, preschool boys are more accurate than girls in spatial tasks and suggests that males develop visual-spatial cognition abilities before females (Levine et al., 1999), these possible development differences do not affect children in any aspect (effectiveness, efficiency, precision) when using the evaluated directional awareness visual languages.

\section{Threats to validity}

Certain precautions should be taken before extrapolating the results obtained in this study to other contexts. Regarding the information on block locations, the arrow and the thumbnail mechanisms do not indicate where the blocks are placed, so the directional awareness mechanisms point to the target regardless of whether there is a block in the path or not. However, the mini-map mode included a small map containing all the elements in the virtual world and the blocks can be seen even though they are off the screen. This could have an impact, since the mini-map technique offers more information and could allow more precise paths. However, despite giving more information, the mini-map technique got the worst results in all the evaluated dimensions. On the other hand, the extra information offered by the mini-map technique may have an impact on the time needed by children to complete the task, since they have to process more information. This limitation should therefore be taken into account when extrapolating the results.

In terms of measuring the changes of direction, each time the children change the direction button while piloting the spacecraft was counted as a change of direction. When they tried to move the character in a diagonal direction (switching between the horizontal and vertical arrows) these switches are counted as changes of direction. This could have some validity issues, since some children may want to drive the spacecraft in a diagonal path and the control interface does not allow it. However, the validity issue regarding the fact that children switch between the vertical and horizontal arrows to move the character diagonally will affect all the techniques in the same way, since 
Kindertivity: Usability and Communicability Strategies for Interactive Surfaces and PreKindergarten Children

the moving mechanism is the same for all the visual languages. In future work it would be interesting to evaluate other indirect dragging techniques, such as a circular dial or a steering wheel to assess their suitability for use by kindergarten children.

Finally, it is worth noting that the American Academy of Pediatrics (AAP Council on communications and media, 2016) recommends avoiding the use of touch screen devices for children younger than 18 months and they also recommend a responsible usage with a limit of one hour of screen per day, with parents co-viewing and collaborating with the children's interactions when they are between two and five years old. It is also important to highlight that the use of touch technology cannot replace essential activities needed by young developing children, such as jumping, running, interacting with others, learning social interaction norms, etc.

\subsection{Conclusions}

In this work we analyzed a corpus of 100 commercial applications running on multitouch devices for kindergarteners in order to determine how these applications use the virtual space. The analysis revealed that most of the evaluated applications are limited to simply using the screen size as the digital world that is always shown and only $33 \%$ of them used a space beyond the screen limits. There are few applications (only $5 \%$ of the revised apps) that use some sort of language to provide directional awareness to users and there is no standard and validated way of providing this information. In order to assess kindergarteners' capacity to use the world beyond the screen boundaries and possible visual languages to communicate directional spatial information, an experimental evaluation was carried out on children aged between 4 and 6 years old in a task requiring the use of a virtual space beyond the screen limits with the assistance of three different directional visual awareness languages.

Our findings provide evidence that even though commercial applications do not use this augmented space, kindergarteners could complete a task requiring directional awareness with success rates of close to 100 per cent. Despite this type of skill being in the process of development, they are ready to interpret and extrapolate the data from the visual languages to locate different objects in a virtual world.

The results revealed that the mini-map was the most problematic technique, whereas the border-floating thumbnails and the arrow techniques reached success rates of over $99 \%$. The techniques evaluated were seen to have different performances in terms of the time needed to complete the task, the relative positioning awareness that they convey and the visual interference that they cause. In this respect, if the completion time is a mandatory requirement of the application, the arrow technique was the fastest, followed by the border-floating thumbnails and the mini-map technique was the slowest. On the other hand, if relative positioning awareness is one of the main requirements, designers should avoid using the mini-map technique, since it was significantly less effective than the arrow and the border-floating thumbnail techniques. 
Finally, when reducing the degree of interference of the visual language with the task is a priority, the most appropriate technique for this is the arrow language, followed by the border-floating thumbnail and the mini-map. This should be taken into account by designers when developing applications in order to choose the most appropriate technique to fit the applications requirements.

No differences were found in the effectiveness, efficiency or language understanding in terms of gender.

Our plans for future work include the design and evaluation of an educational scenario to study the impact of the proposed visual languages in communicating multi-touch gestures (Nacher et al., 2017) and directional awareness in parents' dialogic strategies during the learning process. The goal will be to demonstrate that these visual languages can improve the learning process by helping parents to focus on the learning content rather than on the children's expected interactions. We are also interested in studying whether the results obtained in this study can be extrapolated to a situation in which directional awareness is developed and communicated in a 3D space explored by kindergarteners with the use of digitally augmented tricycles (Tanaka \& Takahashi, 2012) or collaborative robots (Garcia-Sanjuan, Jaen, Nacher, et al., 2015).

\section{Acknowledgments}

This work received financial support from Spanish Ministry of Economy and Competitiveness and was funded by the European Development Regional Fund (EDRF-FEDER) in the project TIN2014-60077-R (SUPEREMOS). This work is also supported by a pre-doctoral fellowship within the FPU program from the Spanish Ministry of Education, Culture and Sports to V. Nacher (FPU14/00136) and from GVA (ACIF/2014/214) to F. Garcia-Sanjuan. 
Kindertivity: Usability and Communicability Strategies for Interactive Surfaces and PreKindergarten Children

\section{Appendix}

Table 23. Success rate by visual language and age group.

\begin{tabular}{l|l|r|r}
\hline Visual language & Age Group & Average & SD \\
\hline \multirow{4}{*}{ Mini-map } & 4 & 86.11 & 29.04 \\
\cline { 2 - 4 } & 5 & 98.89 & 4.97 \\
\cline { 2 - 4 } & 6 & 97.78 & 9.94 \\
\cline { 2 - 4 } & Overall & 94.26 & 18.58 \\
\hline \multirow{3}{*}{$\begin{array}{l}\text { Border-Floating } \\
\text { thumbnails }\end{array}$} & 4 & 100 & 0 \\
\cline { 2 - 4 } & 5 & 100 & 0 \\
\cline { 2 - 4 } & 6 & 99.44 & 2.48 \\
\cline { 2 - 4 } & Overall & 99.81 & 1.43 \\
\hline \multirow{4}{*}{ Arrow } & 4 & 99.44 & 2.48 \\
\cline { 2 - 4 } & 5 & 100 & 0 \\
\cline { 2 - 4 } & 6 & 100 & 0 \\
\cline { 2 - 4 } & Overall & 99.81 & 1.43 \\
\hline
\end{tabular}

Table 24. Completion time by visual language and age group (s).

\begin{tabular}{l|l|r|r}
\hline Visual language & Age Group & Average & SD \\
\hline \multirow{4}{*}{ Mini-map } & 4 & 415.42 & 78.71 \\
\cline { 2 - 4 } & 5 & 367.44 & 97.94 \\
\cline { 2 - 4 } & 6 & 335.46 & 47.53 \\
\cline { 2 - 4 } & Overall & 368.67 & 82.30 \\
\hline \multirow{3}{*}{$\begin{array}{l}\text { Border-Floating } \\
\text { thumbnails }\end{array}$} & 4 & 342.64 & 62.63 \\
\cline { 2 - 4 } & 5 & 304.24 & 51.57 \\
\cline { 2 - 4 } & 6 & 268.00 & 44.55 \\
\cline { 2 - 4 } & Overall & 301.34 & 59.46 \\
\hline \multirow{4}{*}{ Arrow } & 4 & 336.04 & 47.86 \\
\cline { 2 - 4 } & 5 & 313.64 & 66.50 \\
\cline { 2 - 4 } & 6 & 262.79 & 33.00 \\
\cline { 2 - 4 } & Overall & 301.09 & 58.90 \\
\hline
\end{tabular}


Exploring visual languages for communicating directional awareness to kindergarten children

Table 25. Percentage of increment in the travelled path in comparison with the ideal.

\begin{tabular}{l|l|r|r}
\hline Visual language & Age Group & Average & SD \\
\hline \multirow{4}{*}{ Mini-map } & 4 & 27.31 & 16.06 \\
\cline { 2 - 4 } & 5 & 28.09 & 19.81 \\
\cline { 2 - 4 } & 6 & 27.01 & 15.56 \\
\cline { 2 - 4 } & Overall & 27.48 & 17.03 \\
\hline \multirow{3}{*}{$\begin{array}{l}\text { Border-Floating } \\
\text { thumbnails }\end{array}$} & 4 & 25.54 & 16.61 \\
\cline { 2 - 4 } & 5 & 22.50 & 21.04 \\
\cline { 2 - 4 } & 6 & 16.45 & 13.23 \\
\cline { 2 - 4 } & Overall & 21.37 & 17.46 \\
\hline \multirow{4}{*}{ Arrow } & 4 & 19.38 & 11.22 \\
\cline { 2 - 4 } & 5 & 22.02 & 15.64 \\
\cline { 2 - 4 } & 6 & 14.63 & 9.23 \\
\cline { 2 - 4 } & Overall & 18.71 & 12.6 \\
\hline
\end{tabular}

Table 26. Changes of direction by visual language and age group.

\begin{tabular}{l|l|r|r}
\hline Visual language & Age Group & Average & SD \\
\hline \multirow{4}{*}{ Mini-map } & 4 & 16.27 & 4.91 \\
\cline { 2 - 4 } & 5 & 17.85 & 5.83 \\
\cline { 2 - 4 } & 6 & 18.62 & 5.34 \\
\cline { 2 - 4 } & Overall & 17.58 & 5.37 \\
\hline \multirow{3}{*}{$\begin{array}{l}\text { Border-Floating } \\
\text { thumbnails }\end{array}$} & 4 & 16.85 & 4.29 \\
\cline { 2 - 4 } & 5 & 17.37 & 2.94 \\
\cline { 2 - 4 } & 6 & 16.48 & 1.64 \\
\cline { 2 - 4 } & Overall & 16.90 & 3.11 \\
\hline \multirow{4}{*}{ Arrow } & 4 & 16.8 & 3.32 \\
\cline { 2 - 4 } & 5 & 18.12 & 4.67 \\
\cline { 2 - 4 } & 6 & 17.13 & 2.74 \\
\cline { 2 - 4 } & Overall & 17.35 & 3.65 \\
\hline
\end{tabular}


Kindertivity: Usability and Communicability Strategies for Interactive Surfaces and PreKindergarten Children

Table 27. Number of collisions with blocks by visual language and age group.

\begin{tabular}{l|l|r|r}
\hline Visual language & Age Group & Average & SD \\
\hline \multirow{4}{*}{ Mini-map } & 4 & 1.88 & 1.31 \\
\cline { 2 - 4 } & 5 & 1.41 & 1.43 \\
\cline { 2 - 4 } & 6 & 0.97 & 1.04 \\
\cline { 2 - 4 } & Overall & 1.88 & 1.31 \\
\hline \multirow{3}{*}{$\begin{array}{l}\text { Border-Floating } \\
\text { thumbnails }\end{array}$} & 4 & 1.75 & 1.63 \\
\cline { 2 - 4 } & 5 & 1.75 & 1.58 \\
\cline { 2 - 4 } & 6 & 0.73 & 0.72 \\
\cline { 2 - 4 } & Overall & 1.41 & 1.44 \\
\hline \multirow{4}{*}{ Arrow } & 4 & 1.2 & 0.91 \\
\cline { 2 - 4 } & 5 & 1.08 & 1.35 \\
\cline { 2 - 4 } & 6 & 0.62 & 0.68 \\
\cline { 2 - 4 } & Overall & .97 & 1.04 \\
\hline
\end{tabular}

Table 28. Number of incorrect pickups by visual language and age.

\begin{tabular}{l|l|r|r}
\hline Visual language & Age Group & Average & SD \\
\hline \multirow{4}{*}{ Mini-map } & 4 & .98 & .49 \\
\cline { 2 - 4 } & 5 & 1.03 & 1.12 \\
\cline { 2 - 5 } & 6 & .92 & 1.01 \\
\cline { 2 - 5 } & Overall & .98 & .90 \\
\hline \multirow{3}{*}{$\begin{array}{l}\text { Border-Floating } \\
\text { thumbnails }\end{array}$} & 4 & .73 & .54 \\
\cline { 2 - 5 } & 5 & .68 & .58 \\
\cline { 2 - 5 } & 6 & .60 & .53 \\
\cline { 2 - 5 } & Overall & .67 & .54 \\
\hline \multirow{4}{*}{ Arrow } & 4 & .77 & .54 \\
\cline { 2 - 5 } & 5 & .95 & .81 \\
\cline { 2 - 5 } & 6 & .77 & .73 \\
\cline { 2 - 5 } & Overall & .83 & .70 \\
\hline
\end{tabular}

Table 29. P-value of the post-hoc pair-wise comparison of visual languages for all the dependent variables.

\begin{tabular}{l|r|r|r}
\hline \multicolumn{1}{c|}{ Visual languages } & $\begin{array}{c}\text { Mini-map vs } \\
\text { Border-Floating } \\
\text { thumbnails }\end{array}$ & \multicolumn{1}{c|}{$\begin{array}{c}\text { Mini-map vs } \\
\text { Arrow }\end{array}$} & $\begin{array}{c}\text { Border-Floating } \\
\text { thumbnails vs } \\
\text { Arrow }\end{array}$ \\
\hline Success & .061 & .064 & 1.000 \\
\hline Completion time & $<.001$ & $\mathbf{< . 0 0 1}$ & 1.000 \\
\hline Travelled path & .150 & $\mathbf{. 0 0 3}$ & .745 \\
\hline Changes of direction & 1.000 & 1.000 & 1.000 \\
\hline Collision with obstacles & .041 & $<.001$ & .400 \\
\hline Incorrect pickups & .129 & .497 & .628 \\
\hline
\end{tabular}




\section{Chapter 12}

\section{Evaluating Assistive Communication \\ Languages with Kindergarten Children on Touchscreen Devices}

Vicente Nacher, Fernando Garcia-Sanjuan, Javier Jaen

ISSI Group, Dpto. Sistemas Informáticos y Computación, Universitat Politècnica de València.

Submitted to International Journal of Human-Computer Interaction. Under review, as of October 2018.

\footnotetext{
Abstract

A myriad of educational applications using tablets and multi-touch technology for kindergarten children have been developed in the last decade. However, despite the
} 
Kindertivity: Usability and Communicability Strategies for Interactive Surfaces and PreKindergarten Children

possible benefits of using visual languages to communicate information to kindergarteners, these visual techniques have not been fully studied yet. This paper therefore investigates kindergarten children's abilities to understand and follow several visual languages indications about how to proceed and interact in a virtual 2D world. The results show that kindergarteners are able to effectively understand several visual languages with different communication purposes despite being used simultaneously. The results also show that the use of the evaluated visual languages to communicate data when playing reduces the number of interferences about technical nature fostering dialogues related to the learning activity guided by the instructors or caregivers. Hence, this work is a starting point for designing dialogic learning scenarios tailored to kindergarten children.

\subsection{Introduction}

In recent years a great deal of attention has been paid to the use of touch-based devices such as tabletops and tablets. The direct-touch that these devices enable is preferred by children over other mediated pointing devices like the mouse and keyboard, as it provides a more direct way of selecting options on the screen (Hourcade, 2007). Moreover, different studies have pointed out that the multi-touch interaction is a more intuitive way of interaction (Smith et al., 2012) (Jokisch et al., 2011); (Ioannou et al., 2013). Hence, as this technology involves a natural interaction style requiring little training (Fernández-López et al, 2013), tablet-based games have already been tested with children who have demonstrated preference for the educational tablet-based option (Furió et al, 2013). These devices have brought new opportunities to create other forms of interactive media to engage kindergarten children in beneficial educational activities (Nacher, Garcia-Sanjuan, \& Jaen, 2016). With the goal of assessing the suitability of multi-touch tablet devices and to fully exploit its potential to design educational applications targeted to kindergarten children, several works have focused on evaluating the way in which kindergarten children interact with these devices. In this respect, Nacher et al (Nacher, Jaen, Navarro, Catala, \& González, 2015) show that even children aged 2 to 3 are able to perform a basic set of multi-touch gestures (tap, scale up, scale down and rotation) on a tablet without assistance and they are able to perform more complex gestures (such as double tap and long press) when using some assistive techniques (Nacher et al., 2014). In this line, Vatuavu et al (Vatavu et al., 2015) also showed that children aged 3 to 6 are able to perform touch gestures on small devices such as tablets and smartphones. Accordingly, kindergarten children have become frequent users of multi-touch devices such as smartphones and tablets being confronted with this technology even before they fully develop oral communicative functions (Rideout, 2011); (Lydia Plowman et al., 2012); (Cristia \& Seidl, 2015).

However, this growth in the use of multi-touch technology by kindergarten children and the study and evaluation of the gestures that they can successfully perform has not 
been matched with the study of appropriate techniques to communicate information about the applications tailored to their development. Several studies have shown that including instructions in the form of a short text or video clips is suitable for primary school children (Kähkönen \& Ovaska, 2006) (Niemi \& Ovaska, 2007) (Van Der Meij \& Van Der Meij, 2014) but kindergarteners do not have the abilities required to read and understand text messages or complex verbal video instructions. In this sense, the design process of these techniques is especially challenging because kindergarteners are in the process of early language development and the younger they are the more scaffolding of technical nature they need (Neumann, 2017), including these special communication strategies when using touch screen devices. Hence, designers of educational applications targeted to kindergarten children need adequate graphic strategies to enable them to interpret different and diverse information about the applications, such as the gestures to be performed at a given time, the actions needed to go ahead, or information about the spatial location of objects in the virtual world. Therefore, the design of appropriate languages must be addressed since multi-touch interfaces can facilitate dialogic learning scenarios in which the dialog is centered around the learning activity itself rather than on the interactions the children are expected to perform each time (Derboven et al., 2012).

Considering this, designing languages that avoid the need of continuous external technical scaffolding (i.e. the gestures to be performed, the direction in which a game character should move, etc.) is crucial when developing games or applications targeted to young children. The design and usage of visual languages tailored to kindergarten children abilities and development will help caretakers to concentrate more on giving cognitive scaffolding (i.e. the learning content to be acquired by the children) since children will get the other information through the integrated languages. Moreover, in other scenarios in which children can interact collaboratively, the use of visual languages understandable by all the children involved in the game is a key point for them to share information and plan collaboratively the actions to take by referring to visual elements that indicate the possible available actions to perform contributing to a more satisfying and successful group educational experience.

Therefore, in this paper we evaluate several languages in co-existence in a real educational application in order to find out whether the cluttering of different visual languages and several interactive elements in a virtual world has an impact in the understanding of these semiotics with kindergarten children. In addition, we also aim to test whether kindergarten children are ready to use an application which requires sequences of different multi-touch gestures to complete the task with the same success than when performing these gestures in isolation.

The contributions of this work are manifold. The first contribution is the experimental confirmation that kindergarteners are able to effectively understand two different types of visual languages displayed simultaneously and communicating data with several purposes. The second contribution relies on the fact that using visual languages to 
Kindertivity: Usability and Communicability Strategies for Interactive Surfaces and PreKindergarten Children

communicate data about the gestures to be performed and to provide directional awareness fosters dialogues related to the learning activity and reduces the number of interferences about the interaction mechanisms expected each time by the application. The third contribution is the experimental confirmation that despite the task asks kindergarten children to perform sequences of different multi-touch gestures; their performance is not negatively affected. Finally, in this work we have gamified a multimedia application adapting it to kindergarteners' development and skills and the results show that they are ready to use it and that the use of this game fosters dialogues with caretakers about the learning content to be acquired.

\subsection{Related work}

In the literature, several studies evaluating the suitability of multi-touch technology with pre-kindergarten children can be found.

For example, Nacher et al (Nacher et al., 2015) showed that even children aged two and three years old are able to perform properly a basic set of multi-touch gestures including tap, drag, scale (up \& down) and one finger rotation. Moreover, more complex gestures such as double tap and long pressed can also be performed by them when using some assisted strategies (Nacher et al., 2014). The work by Vatavu et al (Vatavu et al., 2015) also pointed out that children aged three to six years old are able to perform the tap, double tap and single hand drag and drop gestures properly. However, not only basic gestures can be done by young children but also gestures that require movement of contacts with high accuracy at the termination of the gesture (such as drag and drop) and more complex gestures (such as the one finger rotation and scales) (Nacher \& Jaen, 2015a) suits with young children's skills. Other studies evaluated the interaction needed to play with four applications (Abdul Aziz et al., 2013, 2014). These applications required the use of gestures such as tap, drag, rotation, drag and drop, pinch, spread and flick. After evaluating them with children aged from 2 to 4 , the results showed that children aged 4 were able to perform all the set of evaluated gestures and those aged 3 only had problems with the spread task. Finally, 2-year-olds were less effective with the more complex gestures such as drag and drop and pinch but were able to perform the tap and drag gestures properly and quickly learnt to perform the flick gesture. Moreover, children's preference for educational tablet-based games has already been demonstrated in real educational applications targeted to transmit knowledge (Furió et al, 2013) because it involves a natural interaction style which requires little training (Fernández-López et al, 2013).

These previous studies reveal that the use of multi-touch technology fits perfectly with young children and point out some guidelines to design and develop the interactions to be included in touch applications specially tailored to kindergarten children skills and development. However, only few and diverse studies can be found addressing the issue of communicability of information to kindergarten children in multi-touch screen devices. Hence, there is no standard way of communicating information to children. In 
this sense several works have evaluated different ways of providing children with instructions about the required interaction. For example, Niemi \& Ovaska (Niemi \& Ovaska 2007), explored an interaction design process with children aged 6 years old when instructions are given and their results showed that instructions in the form of animations to show the correct use of complex tools were best understood but only if these animations were accompanied by spoken instructions. These results are obvious and expected since applications for young children cannot rely on written text to give instructions due to their lack of reading skills. Similarly, animations and written instructions to provide instructions in applications targeted at 5-6 year-old children with desktop computers were explored by Kähkönenet et al (Kähkönen \& Ovaska, 2006). The results showed that despite communicability being especially challenging with young children, following some design guidelines was effective in supporting the communication process and also showed that providing help in the form of audio messages could overcome some of the limitations of written instructions. These guidelines recommended giving visual cues to trigger children's attention in order to help them finding new content and textual instructions adapted for them and providing separated video instruction so that they can focus on a specific explanation. Another example is the work by McKnight and Fitton (McKnight \& Fitton 2010) in which they performed a test on common touch-screen terminology in which English-speaking children aged between 6 and 7 were asked to perform a basic set of touch gestures from audio and written instructions. The obtained results showed most of the instructions were understandable by children and they completed the task easily. Moreover, as can be expected, giving accompanying textual instructions was useful. However, they pointed out that it was hard to establish a consistent link between a specific term and a touch gesture making the design of the given children instructions a critical phase due to their limited vocabulary and reading skills.

Baloian et al. (Baloian et al., 2013) evaluated the use of metaphors to communicate multi-touch gestures to 5-6-year-olds. In their approach, they used metaphors for each of the application's gestures avoiding the use of common names of gestures used by adults. Hence, each gesture was associated to a specific "recallable" character (e.g. a jumping grasshopper for a double tap, a walking ladybug for a drag gesture or a hovering butterfly for a tilting gesture) and these words and pre-recorded audios were used to ask users to identify and perform different gestures. However, the results showed no strong correlations between performance and the behavior of the characters that the children liked or disliked the most.

All the previous works used a combination of text, audio and/or visual cues to give instructions with children aged 5 to 7 and showed that they are ready to use this type of communication despite having some minor issues. However, applications for younger children cannot rely on written text to give information due to the lack of reading skills. Moreover, there is no standardized way to name touch gestures, so using audio cues may present several understandability problems. 
Kindertivity: Usability and Communicability Strategies for Interactive Surfaces and PreKindergarten Children

Other works have addressed the communicability of touch gestures avoiding text and audio cues. Hiniker et al (Hiniker et al., 2015) evaluated prompts such as in-app audio, on-screen demonstrations (with hand demos or changing the visual state of the item) and instructions by an adult model for eliciting gestures such as double tap, horizontal and vertical swipe and shaking the tablet with children aged between 2 and 5 . Their results showed that although the most effective technique was adult guidance, children aged 3 years or older were able to follow other types of cues. Nacher et al (Nacher et al., 2014) (Nacher et al., 2017) analyzed the communicability of three types of touch gestures (in-place, one-contact dynamic \& two-contact points dynamic gestures) comparing three visual languages with children aged 2 to 3 . Despite their results showed that the iconic approach designed for adults was not appropriate for young children, the two animated approaches (using the image of a hand and changing the visual state of the item to be manipulated) had high success rates (reaching 90\%) when communicating gestures which involve movement (drag, rotation \& scales). Hence, these works showed that the basic reasoning related to the interpretation of moving elements on a surface can be effectively performed during early childhood despite the fact that kids develop spatial reasoning during middle childhood (Levine et al., 1999) (Piaget, 1973).

Although the previous results showed that these approaches are feasible and understandable for these users, the studies focus on semiotics for giving instructions to children but not for giving any type of application information, such as spatial information, application goals, etc. Only a recent study has addressed the issue of communicability of spatial awareness about the elements included in a virtual world with children aged between 4 and 7 years old (Nacher, Garcia-Sanjuan, \& Jaen, 2018). Three different visual languages to communicate directional awareness (a mini-map, using thumbnails on the screen borders \& using an arrow to guide the direction in which the main character should be moved) were designed and evaluated. The minimap resulted the most problematic technique, whereas the border-floating thumbnails and the arrow techniques reached success rates of over $99 \%$.

In conclusion, several works have showed that using visual languages is a feasible technique to communicate information about the game/application to young children fostering dialogues about the content and reducing dialogues about the interaction to be performed. However, these works have evaluated these techniques in isolation and in tasks specially designed to test the suitability of the designed languages. In this work we therefore evaluate whether kindergarten children are able to interact/play with applications in which two different visual languages coexist giving cues with information from different sources (i.e. the gestures to be performed and spatial awareness about the digital world of the application). Moreover, the evaluation of the different visual languages is carried out in a real application targeted to kindergarten children. Hence, the results obtained in this work should be a step forward in the process of obtaining effective semiotic systems understandable by kindergarten 
children that could be used in educational applications based on multi-touch technology.

\subsection{Study context}

The overall goal of our study was to test the suitability of using two visual languages simultaneously to communicate different types of information about the virtual world in which kindergarten children have to complete a task by moving a character and performing different sequences of gestures on the objects scattered in the virtual world and to evaluate their effectiveness and efficiency.

Hence, using the GQM (Goal Question Metric) template (Basili et al., 1994), our goal can be defined as follows: analyze the impact of having two different languages with different purposes each one cohabiting in the same virtual world for the purpose of evaluating their suitability from the viewpoint of effectiveness and efficiency in the context of providing both spatial awareness of the objects in a digital game world and the gestures to be performed by kindergarten children to complete the task.

\subsubsection{Visual languages}

In the game, two different visual languages are used to communicate information to children; a visual language designed to give directional awareness and a visual language to point out which gestures are required to complete an action.

The visual language used to point out the required gestures consists of a Mickey Mouse animated hand to represent a hand with one finger extended. Hence, the object to be manipulated with a multi-touch interaction is accompanied by the animated virtual hand (or hands if more than a contact is needed) that provides visual cues about the gesture to be carried out. This animated technique has been previously tested and resulted effective to communicate dynamic gestures such as drag, rotation, scale up and scale down with success rates of up to 90\% with children aged between 2 and 3 years old (Nacher et al., 2017). The gestures to be performed are drag, scale down, scale up and rotation.

On the other hand, the visual language used to give directional awareness to children is a Border-Floating thumbnails technique in which miniatures of the objects that are not visible on the surface appear at the border of the screen. The position where the miniature is shown is the intersection between the vector that links the character to the corresponding object and the screen border (see Figure 77). The miniatures positions are dynamically updated according to the relative character's position at a given moment. With this technique, the visual language only represents the objects that are outside of the current screen display. This technique has been previously evaluated in isolation reaching success rates of over $99 \%$ when guiding young children's movements to reach different targets in a 2D digital world (Nacher et al., 2018). 
Kindertivity: Usability and Communicability Strategies for Interactive Surfaces and PreKindergarten Children

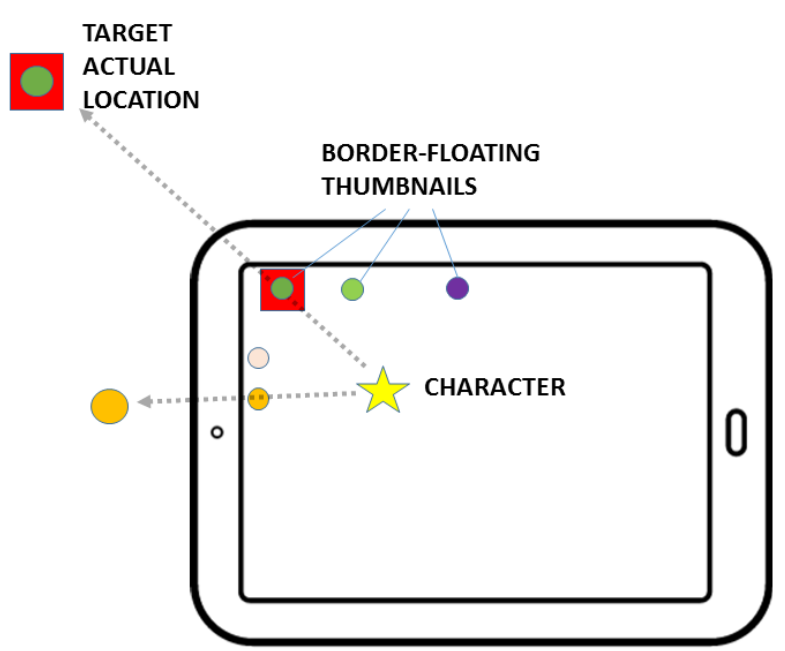

Figure 77. Representation of the thumbnail visual language.

\subsubsection{Participants}

Seventy-five children aged from 4 to 6 years old took part in the experiment (Mean $(\mathrm{M})=60.24$ (months), Standard Deviation $(\mathrm{SD})=6.5$ ) with a gender distribution of 34 males and 41 females.

The children were split up into two balanced age groups, i.e. they were grouped by age, with each age group comprising the ages [4, 5[ and [5,6[. The distribution of the age groups were 39 children in the 4-years age group and 36 children in the 5-years age group.

The 4 to 6 year age range was chosen in order to assess whether the usage of different type of visual languages working at the same time and the requirement of different types of gestures to be performed in sequence for different purposes is affordable for children in the earliest stage of development. The youngest users were children aged 4 years because in previous studies (Nacher, Ferreira, et al., 2016), children were found not to be able to move/guide a character in a $2 \mathrm{D}$ world using indirect drag techniques with acceptable success when they are aged less than 4 . Parental authorization was obtained before carrying out the study.

\subsubsection{Equipment}

The interaction framework for the experiment was implemented in Java using the LibGDX framework. The devices used for the experiment were BQ Aquaris M10 tablets with Android 5.1. The tablets were equipped with capacitive multi-touch screens with a $1280 \times 720$ px resolution. 


\subsubsection{Task}

The task designed is in context with the educational goals of the school where the activity took place. In this respect, teaching animal conservation and care topics was an educational goal for children in this early childhood. This educational theme is becoming more and more important because children are infrequently in touch with the natural world as pointed out by (Willis, Weiser, \& Kirkwood, 2014). Hence, educating on environmental care values is necessary for children to start developing responsible behaviors regarding the natural environment (Louv, 2008). Environmental education is usually addressed through the basic premises of scientific inquiry: exploring, observing, communicating, organizing, applying, relating and inferring (Arce, 2013) so the task has been designed to evaluate children' preferences and engagement when taking part in activities of different nature. The task has been split up in three different stages corresponding to different cognitive processes educators wanted to develop. The first one relies on an adventure game style in which free exploration of a virtual world is allowed fostering curiosity and discovery. In this part of the task children explore a 2D world simulating a natural ecosystem with different animals scattered around the digital world. The second part of the task consists in a problem-solving reasoning activity in which children have to "help" a specific animal performing different care actions. Finally, in the third stage of the task, children will be able to carry out an observation activity by visualizing a real video of the type of animal that has been cared.

In the following, each stage of the game is explained in detail and, in addition, an example of the game usage with these three stages has been recorded in order to facilitate the task explanation ${ }^{12}$.

\section{Exploration \& Curiosity}

The first part of the task relies on an adventure game style in which children have to control the movements of a character to explore the available open 2D space in search of animals to take care of. The choice of this type of game style is because it stimulates curiosity (Collins \& Stevens, 1981) (Malone, 1981) and can potentially facilitate a range of different learning styles such as tutoring, practice and self-learning (Dempsey et al., 1994). Moreover, this type of activity fosters learning discovery which is a technique that helps learners to create and organize their knowledge, since they draw upon past knowledge and experience to infer underlying strategies and gain understanding of concepts (Honomichl \& Chen, 2012). Knowledge discovery is also beneficial for students' motivation, since those who discover information for themselves are more motivated to achieve educational goals and more likely to remember the information learned (Bruner, 1960). Taking this into account, the task

\footnotetext{
12 https://goo.gl/trjB2i
} 
Kindertivity: Usability and Communicability Strategies for Interactive Surfaces and PreKindergarten Children

begins in the Exploration \& Curiosity stage in which children can move a vehicle in a virtual 2D world (see Figure 78). The vehicle can be moved using four arrow-shaped buttons symbolizing the four basic directions (i.e. up, down, left and right). These buttons are placed at the bottom-center of the screen and allow users to move the target by tapping and holding one finger on the button that symbolizes the desired direction. This indirect drag technique has been evaluated previously showing that children aged 4 years and older are able to use this indirect drag technique with high success and reducing the number of undesired collisions respect to other indirect techniques (Nacher, Ferreira, et al., 2016).

In the task, children move the vehicle over the virtual world in order to visit the animals they want to help and watch. In order to develop problem-solving skills educators proposed that the animals should be surrounded by water that the vehicle cannot cross; however, malleable bridges are placed in each water point (see Figure 78). These bridges need to be rotated and/or scaled in order to fit with the water size and shape. This task was also proposed by educators in order to develop geometric interpretation skills. Once the bridge is fitted the water disappears and the vehicle can go through to reach the animal. The gestures to be performed in order to fit the bridges are scale down, scale up and rotation. Some bridges require only one of these gestures to be fitted and others need a sequence of them (rotation and scale up/down) to be fitted. The visual language about the gestures to be performed will only be visible when the main character is close to the bridge location in which case the bridge will be enabled to be manipulated.

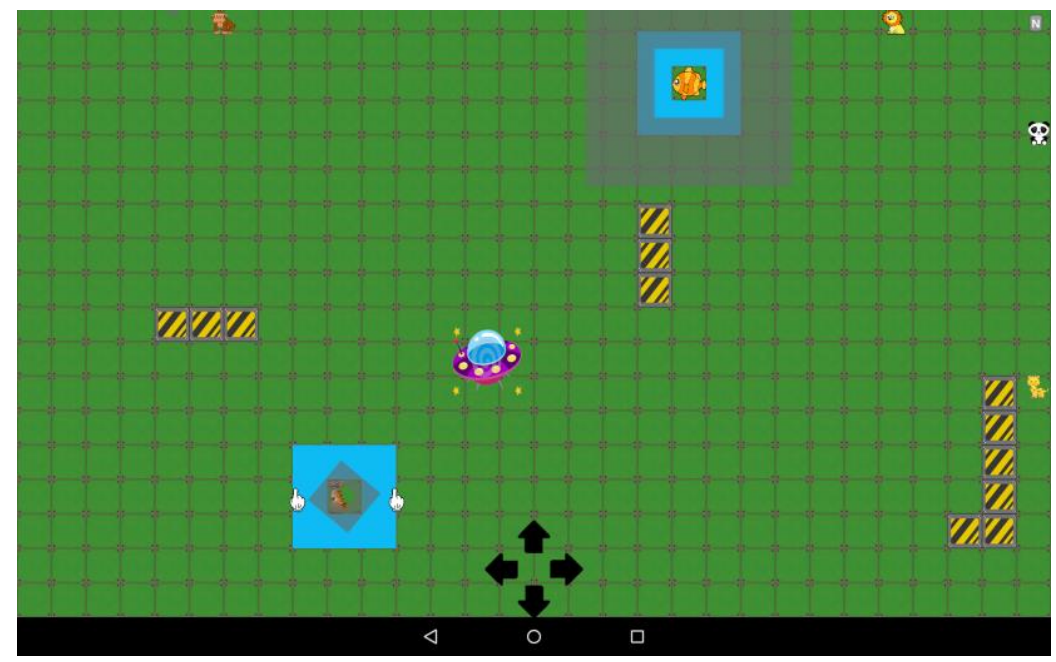

Figure 78. Example of the game in the Exploration \& Curiosity stage with an active bridge and the visual language representing a scale up gesture. 
In order to evaluate the interface under two different levels of visual information density (low and high), two different scenarios varying the information density are used. The first one corresponds to a setting with low density, hence, three animals to be cared of are used. The second setting has six animals to visit being a scenario with a high visual information density. Each child is randomly assigned with one setting.

When the vehicle finally reaches the location of an animal, the second stage of the game is launched in which children have to take actions to care this specific animal.

\section{Problem-solving Reasoning}

In this stage, children are told to take care of an animal who is not feeling well by giving it food, medicine or cleaning it. Children aged between 4 and 5 years begin to understand inference as a source of knowledge and around the same time they evidence an understanding of knowledge gained through perception and communication (Keenan, Ruffman, \& Olson, 1994), hence, this part of the task aims to help children to infer that taking the appropriate medicines, having good hygienic habits and having a proper diet will make them healthy. The design of the activity resulted from a discussion with educational experts who decided that a matching activity should be proposed at this stage. Therefore, in this part of the game, three images of the animal, the one reached in the previous exploration stage, appear on the right side of the screen and three different images representing care actions to be dragged appear on the left side. The three images of the animal are tailored to represent three different states; a sick animal, a hungry animal and a dirty animal (see Figure 79).

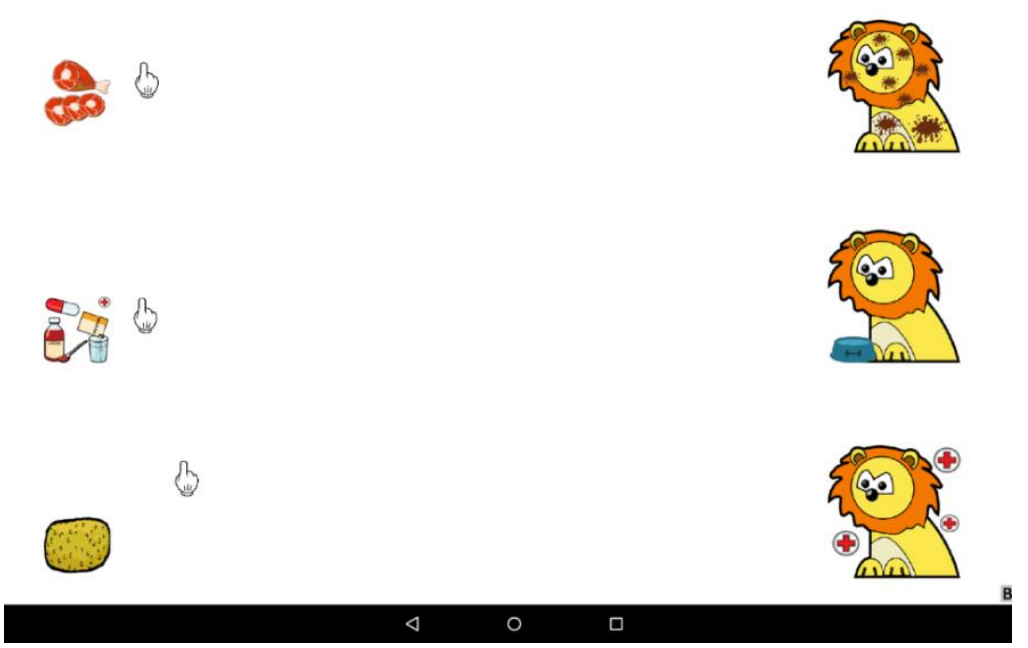

Figure 79. Example of the game in the Problem-solving Reasoning stage for the lion.

The three images to be moved are objects that the animal needs; medicines, food and a sponge to be cleaned. These objects have to be matched with the animal that requires 
Kindertivity: Usability and Communicability Strategies for Interactive Surfaces and PreKindergarten Children

the corresponding action. An animated semiotic using a moving hand is used to point out to children that a drag interaction is needed. Once an object is brought to the correct animal, the characterization disappears and the animal appears with a green background denoting that it is fine (healthy, clean or without hunger). When the three images are matched with the corresponding image of the animal, a video of the animal is automatically played in full screen mode starting the third phase of the game.

\section{Observation}

Children are able to learn from video visualization since the early childhood (Allen \& Scofield, 2010; Pecora, Murray, \& Wartella, 2009), hence, in this stage of the task, children visualize a video related to the animal that they have helped previously (see Figure 80). Children are able to quit the video whenever they want and they are able to go forward or backward in the video as they wish. With the help of educators, the videos were previously selected when designing the game from a set of available videos in the Youtube platform.

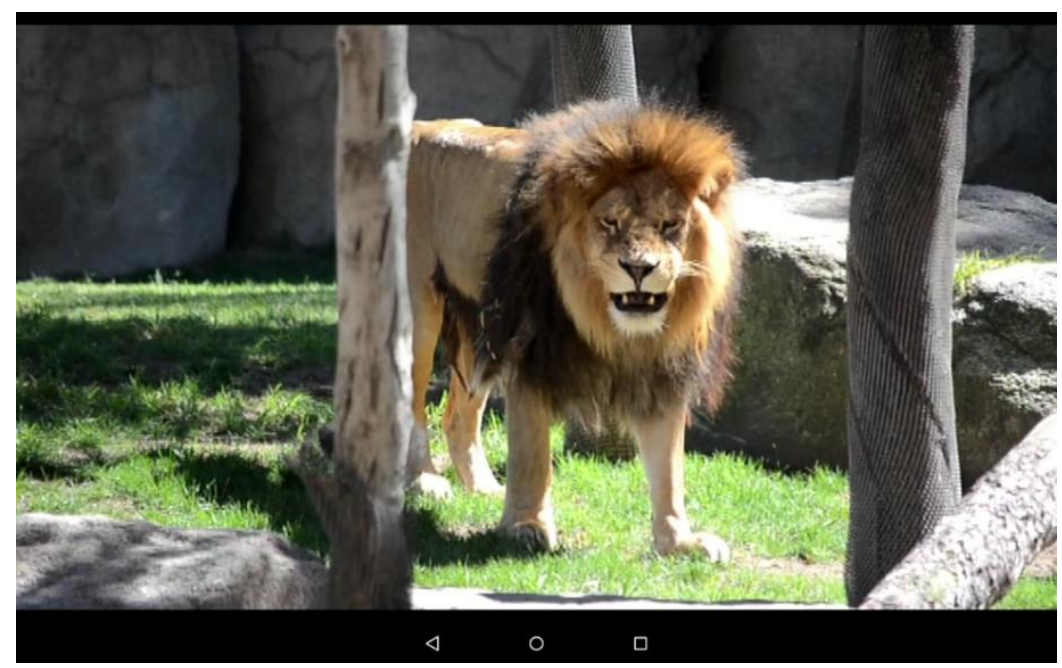

Figure 80. Example of the Observation stage for the lion.

\subsubsection{Procedure}

The children participated in the experiment one day. At the beginning, the instructors explained the task to children telling them that the task consists of a game in which they have to move a vehicle to visit and take care of different animals that appear in the game and, then, as a reward visualize a video of the animals after being helped. The children were given a 1-minute learning phase with an instructor in order to explain the button based technique to move the vehicle and the visual languages used (i.e. the border-floating thumbnails technique for the spatial awareness and the moving-hands technique to communicate the gestures to be performed). Then the game begins in the 
Explore stage and children are free to visit (or not) the animals they want. Each child plays in a setting randomly assigned so they have three or six animals to visit depending on the setting assigned. An instructor is accompanying children all the time and can eventually help them if they ask for it. Around 15 minutes are given to each children to play the game. The experimental platform registered the times dedicated by the children in each of the three stages (Exploration \& Curiosity, Problem-solving Reasoning and Observation), the number of times a given animal is visited and the gestures performed by children to fit the bridges. Moreover, a qualitative analysis is also carried out from the notes taken by the instructors during the experimental sessions.

\subsubsection{Research questions}

The research questions of this work are formulated as follows. The first research question is about the engagement of the game:

- RQ1: Is the game attractive to children and engage them along the duration of the task?

Then, five research questions are stated and will be answered for each factor $F_{i}$ considered (where $i=$ Semiotic density, Age and Gender):

- RQ2: Is the time spent in the Exploration \& Curiosity stage affected by the factor $\mathrm{F}_{\mathrm{i}}$ ?

- RQ3: Is the time spent in the Problem-solving Reasoning stage affected by the factor $F_{i}$ ?

- RQ4: Is the time spent in the Observation stage affected by the factor $\mathrm{F}_{\mathrm{i}}$ ?

- RQ5: Is the spatial exploration scope affected by the factor $\mathrm{F}_{\mathrm{i}}$ ?

- RQ6: Is the effectiveness of the gestural semiotic affected by the factor $F_{i}$ ?

\subsubsection{Design}

Six dependent variables were defined: percentage of Explore \& Curiosity time, percentage of Problem-solving Reasoning time, percentage of Observation time, percentage of the available animals visited, total animals visited and percentage of gestures correctly performed. A between-subject ANOVA (with an $\alpha=0.05$ ) was carried out with the factors: semiotics density with two levels (low vs high), age group with two levels (4 years vs. 5 years) and gender (Male vs. Female).

\subsection{Results}

\subsubsection{Time dedicated by stage}

With the purpose of evaluating children preferences when playing, the time spent by each user in each of the three stages of the game were registered. The time spent in 
Kindertivity: Usability and Communicability Strategies for Interactive Surfaces and PreKindergarten Children

each stage respect to the total time of the task is expressed as a percentage in Figure 81 and the times by each of the factors evaluated are shown in Table 31, Table 32 and Table 33.

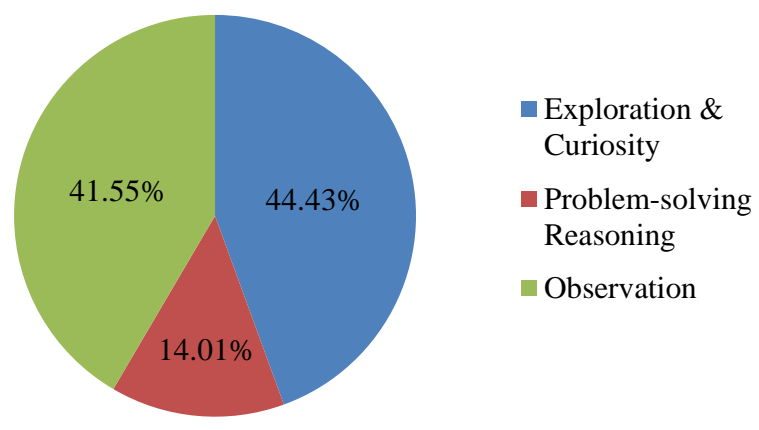

Figure 81. Time spent in each stage of the task in percentage.

A three-way between-subject ANOVA with the independent variables gender, age group and semiotic density and the dependent variables percentage of Exploration \& Curiosity time, percentage of Problem-solving Reasoning time and percentage of Observation time was applied. The statistical analysis revealed significant differences in the between-subject factors age group and semiotic density (see Table 30). The differences between the age groups in the distribution of time can be seen in Figure 82, children aged 4 years spent significantly more time than those aged 5 years in the Exploration \& Curiosity stage $\left(\mathrm{M}_{4 \text {-years }}=48.40 \%\right.$ vs $\left.\mathrm{M}_{5 \text {-years }}=40.03 \%\right)$. However, those aged 5 years, spent significantly more time visualizing the videos than the younger age group $\left(\mathrm{M}_{4 \text {-years }}=37.31 \%\right.$ vs $\left.\mathrm{M}_{5 \text {-years }}=46.26 \%\right)$. No differences were found in the Problem-solving Reasoning stage with both age groups spending around a $14 \%$ of the total time.

On the other hand, the semiotic density has an impact too in the time distribution between the stages being significantly higher the time spent in the Observation stage when the semiotic density was low (i.e. three animals are available) $\left(\mathrm{M}_{\mathrm{low}}=44.79 \%\right)$ than when it was high (i.e. six animals were disposed in the game) $\left(\mathrm{M}_{\text {high }}=39.07 \%\right)$. Moreover, as it was expected, the time spent by children in the Explore \& Curiosity stage was significantly lower when the semiotic density was low $\left(\mathrm{M}_{\text {low }}=41.46 \%\right)$ than when it was high $\left(\mathrm{M}_{\text {high }}=46.72 \%\right)$ (see Table 30). No differences were found between the two semiotic density settings in the Problem-solving Reasoning stage of the game.

Finally, the analysis demonstrated that the time spent in each stage of the game was not significantly influenced by gender (see Table 30 ). 


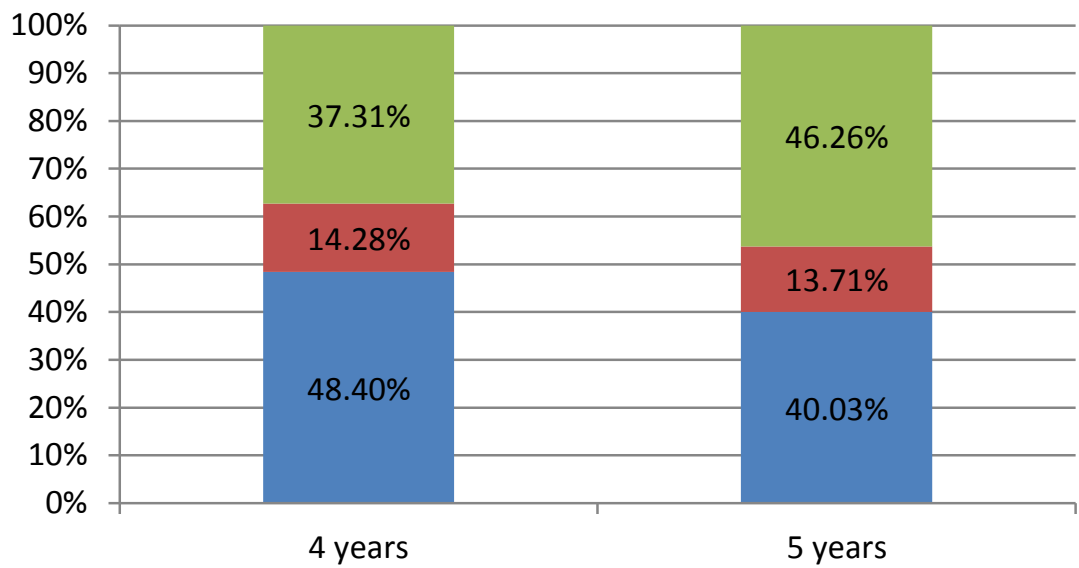

Exploration \& Curiosity $\square$ Problem-solving Reasoning $\square$ Observation

Figure 82. Percentage of time spent in each stage by age group.

\subsubsection{Spatial exploration scope}

To evaluate the effectiveness of the spatial awareness semiotic and its ability to effectively communicate the relative position of different targets with respect to the current position of the main character, the number of times that an animal is visited was registered.

\subsubsection{Virtual space covered}

In order to assess the effectiveness of the semiotic to provide spatial awareness of all the animals scattered along the virtual world, the percentage of available animals that has been visited during the task is depicted in Figure 83 by age group, semiotic density and gender. The detailed data can be seen in Table 31, Table 32 and Table 33.

The ANOVA did not reveal significant effects on the percentage of available animals visited of the gender and age group factors (see Table 30). On the other hand, the statistical analysis revealed significant effects on the percentage of the available animals visited of the factor Semiotic density, being the percentage $100 \%$ when it was low (i.e. three animals were available in the task) and $90.70 \%$ when the semiotic density was high (i.e. six animals available to visit). However, despite this difference, the average percentage of the available animals that have been visited during the task is over $90 \%$ showing that the spatial awareness semiotic fulfills effectively its function communicating to children where the animals are located in the virtual open 2D world independently of the semiotic density. 
Kindertivity: Usability and Communicability Strategies for Interactive Surfaces and PreKindergarten Children

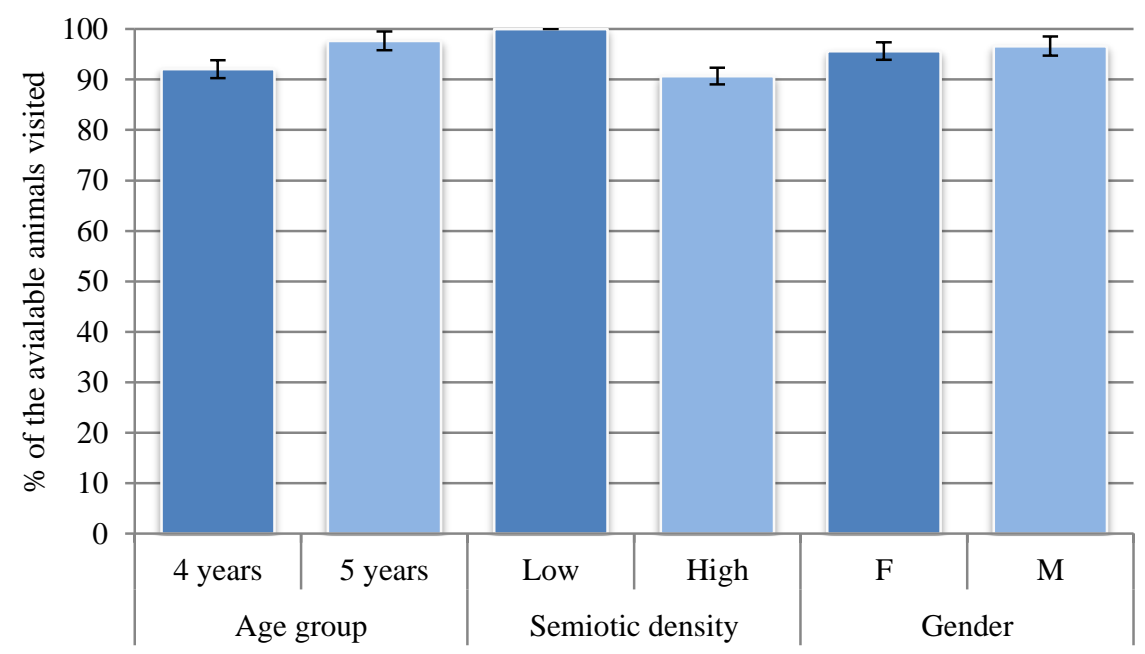

Figure 83. Percentage of the available animals visited during the experiment by age group, semiotic density and gender.

\subsubsection{Exploration efficacy}

The total number of animals visited was analyzed too in order to assess the efficacy of the language to reach different points of the virtual game several times and to find out whether children revisit elements of interest in a fifteen minutes play. The total number of animals visited is shown in Figure 84 by age, gender and semiotic density.

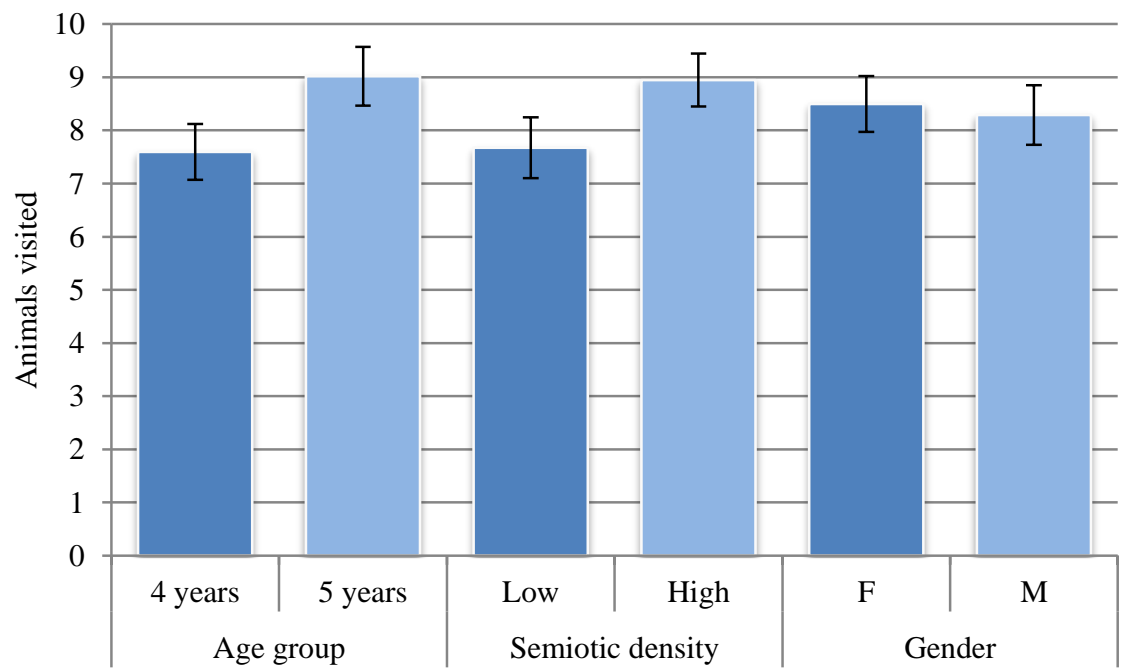

Figure 84. Total animals visited in the task by age, semiotic density and gender. 
The analysis revealed that none of the evaluated factors (age, semiotic density and gender) have a significant impact on the total number of animals visited by children during the task (see Table 30). On average they visit a number of animals between seven and nine in the fifteen minutes that they were playing with the game, hence, children visited an animal approximately every two minutes of playing. This data shows that kindergarten children are ready to complete the task without having big handicaps.

\subsubsection{Gestural interaction language effectiveness}

In order to assess whether the need to perform sequences of gestures has an impact on the success rates of children and whether the language used to communicate the gestures to be performed is effective with kindergarten children when the virtual space is also cluttered with the directional awareness visual language, the sequence of gestures to be performed by children for each "bridge" was predefined in order to compare the total number of gestures fitted by each children (i.e. 4 gestures were needed to be performed to fit all the bridges when the semiotic density was low and 8 gestures were needed when the semiotic density was high). Hence, the percentage of gestures fitted respect to the total number of gestures previously set is evaluated. This percentage is shown in Table 31, Table 32 and Table 33 and graphically in Figure 85.

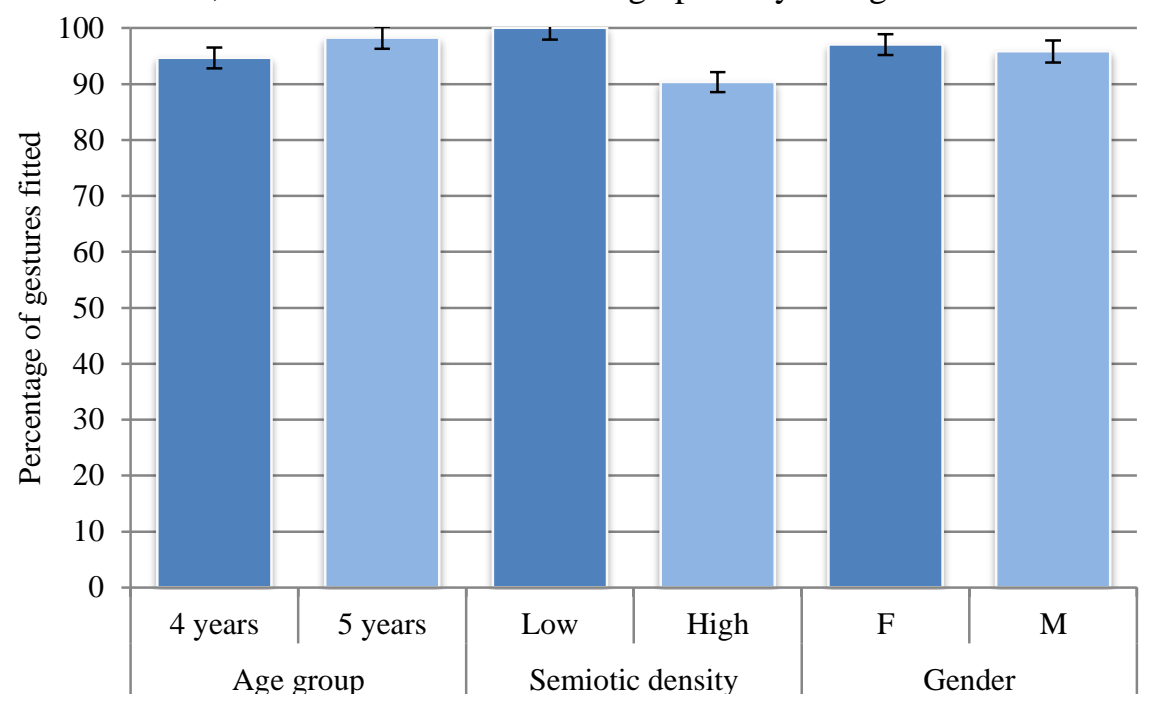

Figure 85. Percentage of gestures fitted by age, semiotic density and gender.

The ANOVA revealed significant effects on the percentage of gestures fitted of the factor Semiotic density being the percentage $100 \%$ when it was low and $90.34 \%$ when it was high. However, despite the differences, both settings have a percentage of gestures fitted over $90 \%$. Hence, children are able to perform the evaluated gestures in sequences with high success rates and the languages used to point out the required 
Kindertivity: Usability and Communicability Strategies for Interactive Surfaces and PreKindergarten Children

gestures to be performed to fit the bridge is still effective in co-existence with the directional awareness language.

Moreover, the analysis revealed that the age group and gender factors did not have a significant impact in the percentage of gestures fitted (see Table 30).

Hence, the results show that kindergarteners are able to successfully perform sequences of different basic multi-touch gestures and they understand the language even when several interactive objects requiring different gestures are placed in the virtual world and when the interference of the directional awareness language is present.

\subsubsection{Observational findings}

In addition to the automatic data logging, throughout the experiment sessions, notes were taken about children's actions, dialogs and behaviors while interacting with the application. These observational findings are not quantified since the impossibility of recording the sessions prevents us from reporting precise measures but valuable information regarding the behavior of children during the experiments can be extracted from them.

The observation revealed that the game fostered different types of conversations. For example, while playing the game, children talked to the instructors to explain them some issues related to the game topic (e.g. "The panda likes to eat bamboo", "The lion is dirty because he has played in the mud"), talked about their future actions (e.g. "Now I am going to help the panda because he is sad", "I am going to visit the lion and then the giraffes"), sometimes they ask for help or ask for permission to do something (e.g. "How do I have to clean the lion?", "Can I visit the gorilla again?"), and, finally, children also make comments about being a doctor or a vet in the future; they also talked about their pets, and some of them also recognized the zoo infrastructures and talked about previous visits to it with their family or school.

On the other hand, one of the most observed children's actions during the experiment was to ask for more animals when they have visited all the available ones of the task. This situation usually happened when children played with the low semiotic density game setting (i.e. three animals available to visit). However, despite asking for more animals to watch, none of the participants wanted to leave the task before they spent all the time given by instructors. This fact points out that using a high semiotic density does not have negative effects in children perception of the game but the more animals to visit the more engage and fun perceived by children.

The observation also reports that most children wanted to visit all the available animals in the game as fast as possible. Hence, children usually left the video observation stage as a secondary task and, usually, they visualized only a short part of the video and went back to the Exploration \& Curiosity stage in order to guide the vehicle to all the available animals. However, once children had visited all the animals, helped them and realized that there were no more animals to visit; they revisited the animals that they 
liked the most and spent more time watching the videos and asking or speaking about them.

Another frequently observed action was children speaking to the animals. They usually speak to the animal they want to help (e.g. "Hey giraffe! Take your food, I will clean you and I will give you your medicines", "I am going to take care of you because you have a cold", "It's done gorilla, now you are fine!") showing that they empathize with the animals and try to help them.

Finally, most of the children asked for playing more time or expressed their feelings about the game time being too short since they wanted to play more. This happened independently of the stage of the game in which they were in that moment; if the time run out when a children was in the Exploration \& Curiosity stage they used to ask for visiting the last animal, if the time ended in the Problem-solving Reasoning stage they asked for additional time to finish the care actions that they were doing, and, finally, if the time ended in the Observation stage children used to ask for additional time to finish watching the video.

\subsection{Discussion}

The experiment explored and answered the comprehensive set of research questions that had been posed. The first research question (RQ1) about whether kindergarten children found the game engaging and played along all the time predefined for the experimentation is affirmatively answered as none of the children left the task before the time limit. Children used all the available time playing and interacted with the application until the instructors ask them to stop. Although the experimentation showed that children find more attractive visiting new animals each time than revisiting animals previously reached, they continued playing when they were told that there were no more different animals to visit and they invested more time in the Observation game stage. Moreover, many children asked for playing a bit more showing that the game resulted engaging and fun for them.

If we consider RQ2 in terms of the time spent in the Exploration \& Curiosity stage, it is answered affirmatively for the factors Semiotic density and Age. The results showed that when playing with a low semiotic density setting, children spent less time in the Exploration \& Curiosity stage $\left(\mathrm{M}_{\mathrm{low}}=41.46 \%\right.$ vs $\left.\mathrm{M}_{\text {high }}=46.72 \%\right)$. It is an expected result since the more interactive elements scattered in the virtual world the more time children need to reach all of them. Regarding the Age factor, the results showed that younger children aged 4 years spent significantly more percentage of the total time than those aged 5 years in the Exploration \& Curiosity stage $\left(\mathrm{M}_{4 \text {-years }}=48.40 \%\right.$ vs $\mathrm{M}_{5 \text { - }}$ years $=40.03 \%$ ). This happens since younger children spent less time in the Observation stage of the game as they were less developed and have a more limited attention span (Hanna, Risden, \& Alexander, 1997), they lose the attention in the video visualization 
Kindertivity: Usability and Communicability Strategies for Interactive Surfaces and PreKindergarten Children

activity and come back to the Exploration \& Curiosity stage earlier than the older age group.

RQ3, on whether the time spent in the Problem-solving Reasoning stage is affected by any of the evaluated factors, is answered negatively for all the factors. As expected, the Semiotic density does not have an impact in this stage of the game since only the latest visited animal appears independently of the game density setting. Regarding the Age factor, it can be expected that no differences between the two age groups will arise in terms of usability since the task is based on performing drag gestures with precision in the termination phase of the gesture. Previous studies have showed that even children aged 2 years old are able to perform this type of gesture with high accuracy (Nacher \& Jaen, 2015b). Moreover, the results showed that the cognitive load of performing the matching activity is equally overcome by children in both age groups since no time differences were found between them when performing this part of the task. This is consistent with previous literature which shows that matching and sorting activities are feasible for young children since the age of three (Pemberton \& Road, 2009).

Regarding the RQ4 on whether the time spent in the Observation stage is affected by any factor, it is affirmatively answered for the factors Semiotic density and Age. The results showed that when playing with a high semiotic density setting, children spent less time than when they play with a low density. As reported in the observational results, children prioritized visiting all the animals to help them. Hence, they left the video observation stage in a second level of priority. Hence, when playing with a high semiotic density, children required and used more time to visit all the animals in the game and the time remaining when all the animals were visited was lower than when a low semiotic density was set. This prevented children to invest much time watching the videos. Regarding the Age factor, the results showed that the youngest age group (those aged 4 years) spent significantly less time than the older group in the Observation stage of the task. This is the case because the more developed children are, the more patience they have being able to wait watching the video and avoiding the eagerness of the younger. This is consistent with the literature which shows that preschoolers have a limited attention span which is increased with age (Hanna et al., 1997) and get distracted too quickly (Egloff, 2004). Consequently, the less developed children used to get distracted when visualizing the videos and returned to the Exploration \& Curiosity stage in order to interact with the game.

In response to RQ5, on whether the spatial exploration scope is affected by any factor, it is answered negatively for the Age factor in terms of the virtual space covered and the exploration efficacy. The absence of significant differences in terms of the virtual space covered shows that the effectiveness of the language to provide spatial awareness of all the animals scattered along the virtual world is high and, despite development differences, no differences were found between groups (above $90 \%$ of the virtual elements reached for both age groups). In terms of exploration efficacy, the results showed that the language was equally effective with both age groups when helping 
them to reach different points of the virtual game repeatedly. On the other hand, the RQ5 is answered affirmatively for the factor Semiotic density in terms of the virtual space covered since the results showed that when the semiotic density was low children visited a higher percentage of the available cameras (100\%) than when the density was high $(90.70 \%)$. However, despite these significant differences the effectiveness of the semiotic when providing spatial awareness of all interactive elements scattered along the virtual world was high for both settings and the differences may appear because children do not have the required time to visit all the animals when a high density was set and not due to a communication problem. Hence, these results show that the spatial directional awareness semiotic can be used in both scenarios (i.e. scenarios with low or high density of interactive elements). In terms of the exploration efficacy, no significant differences were revealed in the Semiotic density factor since children visited a similar number of animals independently of the setting. Hence, cluttering a virtual space with several interactive elements does not have a negative impact on the effectiveness of the spatial awareness visual language so it can be used in scenarios with both, low and high density of interactive elements.

If we consider RQ6 in terms of the effectiveness of the gestural semiotic, then RQ6 is negatively answered for the Age factor. The effectiveness of the gestural semiotic has been previously tested and resulted effective when communicating dynamic gestures to children aged 30 months and elder (Nacher et al., 2017). However, this result shows that despite the usage of the semiotic simultaneously with another visual language the effectiveness is not affected negatively and no differences between children aged 4 and 5 were found. Moreover, the need of communicating sequences of gestures did not have a negative impact on the performance in none of the age groups. Regarding the Semiotic density factor, significant differences were found between the two settings. However, in both settings children performed over the $90 \%$ of the gestures to be done to fit the bridges. This result is interesting since, firstly, it shows that children are able to perform sequences of basic touch gestures without having a negative impact on the success rate and, secondly, it shows that children are able to effectively understand the language to communicate gestures when being displayed simultaneously with another visual language and they are able to interpret sequences of communications of different gestures without major issues.

Finally, regarding the impact of gender, the results showed that in general there were no differences between males and females in any of the evaluated dependent variables, so that all the research questions (RQ2, RQ3, RQ4, RQ5 and RQ6) are answered negatively for the Gender factor. This is an interesting result since it shows that even though previous studies with pre-kindergarten children have found that, on average, preschool boys are more accurate than girls in spatial tasks and suggests that males develop visual-spatial cognition abilities before females (Levine et al., 1999). These possible development differences do not affect children in any aspect (effectiveness of the visual languages, time spent distributions, gesture usability) when interacting with the game. 
Kindertivity: Usability and Communicability Strategies for Interactive Surfaces and PreKindergarten Children

\subsection{Conclusions}

In this work we have evaluated the suitability of using two different types of visual languages displayed simultaneously and communicating information with several purposes in applications targeted to kindergarten children. The goal was to find out whether this approach is feasible with this specific type of user and to preliminary assess the usefulness of these visual languages for giving technical support when interacting (giving cues about which gestures need to be performed and giving cues about the location of the different interactive elements placed in the virtual world.

The results confirmed that kindergarteners are able to effectively understand these visual languages despite being used simultaneously and they are able to interact with the application without major issues. Moreover, the evaluation has been carried out with an actual game with several activities and the results showed that children effectively achieved the game goals.

On the other hand, the evaluation also has shown that the use of visual languages to communicate information about the gestures to be performed and to provide directional awareness reduces the number of interferences about technical nature of the game (i.e. explain the gestures needed to complete the task or the interactive elements location in the virtual world) fostering dialogues related to the learning activity guided by the instructors or caregivers.

In addition, the experimentation revealed that kindergarten children are able to perform sequences of touch screen gestures with the same success that they have when performing them in isolation.

Our findings also showed that no differences were found in the times spent in each stage of the game by children and in the efficacy of the visual languages in terms of gender.

Finally, the application tested in this work is a gamified version of a multimedia application targeted to hospitalized children aged between 8 and 18 years and the results showed that it fits with kindergarteners' development and skills, they found it engaging and fun and dialogues with caretakers about the learning content to be acquired are fostered. In this respect, our plan includes the evaluation of this game with actual hospitalized children who are not able to leave their bed for long periods of time and are not prone to engage in conversations in order to assess whether the application usage promotes dialogues between children and their caregivers in an isolated sterile room or even with children in contiguous beds if the application is used collaboratively.

Lastly, it would also be worth evaluating other applications with different purposes and more cognitively complex tasks to assess whether prekindergarten children are able to understand the different visual languages in other contexts. 
Evaluating Assistive Communication Languages with Kindergarten Children on Touchscreen Devices

\section{Acknowledgments}

We would like to thank the CEIP Vicente Gaos primary school for their collaboration during the development of this study.

\section{Funding}

This work is supported by the Spanish Ministry of Economy and Competitiveness and funded by the European Development Regional Fund (EDRF-FEDER) with Project TIN2014-60077-R; by VALi+d program from Conselleria d'Educació, Cultura i Esport (Generalitat Valenciana) under the fellowship ACIF/2014/214, and by the FPU program from Spanish Ministry of Education, Culture, and Sport under the fellowship FPU14/00136. 
Kindertivity: Usability and Communicability Strategies for Interactive Surfaces and PreKindergarten Children

\section{Appendix}

Table 30. Statistics of the conducted ANOVA for all the dependent variables.

\begin{tabular}{l|c|c|c|c|c|c|c}
\hline \multirow{2}{*}{ Dependent Variable } & \multirow{2}{*}{ DoF } & \multicolumn{2}{|c|}{ Gender } & \multicolumn{2}{c|}{ Age group } & \multicolumn{2}{c}{ Semiotic } \\
\cline { 3 - 8 } & & $\mathbf{F}$ & p-value & F & p-value & F & p-value \\
\hline \% of Exploration \& Curiosity time & $(1.76)$ & .934 & .337 & 8.137 & .006 & 5.317 & .024 \\
\hline \% of Problem-solving Reasoning & $(1.76)$ & 2.120 & .150 & .376 & .542 & .260 & .612 \\
\hline \% of Observation time & $(1.76)$ & 1.822 & .181 & 7.422 & .008 & 4.871 & .031 \\
\hline \% of cameras visited & $(1.76)$ & .424 & .517 & 4.230 & .044 & 11.433 & .001 \\
\hline Total cameras visited & $(1.76)$ & .002 & .962 & 3.373 & .071 & 2.764 & .101 \\
\hline \% of gestures fitted & $(1.76)$ & .158 & .693 & 1.654 & .203 & 4.609 & .035 \\
\hline
\end{tabular}

Table 31. Value of the evaluated dependent variables by age group.

\begin{tabular}{l|l|r|r}
\hline Dependent variable & Age Group & Average & \multicolumn{1}{l}{ SD } \\
\hline \multirow{3}{*}{ Exploration \& Curiosity time } & 4 & 48.40 & 11.30 \\
\cline { 2 - 4 } & 5 & 40.03 & 14.02 \\
\cline { 2 - 4 } & Overall & 44.43 & 13. \\
\hline \multirow{3}{*}{ Problem-solving Reasoning time } & 4 & 14.28 & 3.99 \\
\cline { 2 - 4 } & 5 & 13.71 & 5.43 \\
\cline { 2 - 4 } & Overall & 14.01 & 4.7 \\
\hline \multirow{3}{*}{ Total camera visited } & 4 & 37.31 & 12.24 \\
\cline { 2 - 4 } & 5 & 46.26 & 16.27 \\
\cline { 2 - 4 } & Overall & 41.55 & 14. \\
\hline \multirow{3}{*}{$\%$ of available cameras } & 4 & 7.60 & 2.85 \\
\cline { 2 - 4 } & 5 & 9.31 & 3.58 \\
\cline { 2 - 4 } & Overall & 8.41 & 3.3 \\
\hline \multirow{2}{*}{ of gestures fitted } & 4 & 92.08 & 15.09 \\
\cline { 2 - 4 } & 5 & 97.69 & 7.07 \\
\cline { 2 - 4 } & Overall & 94.74 & 12. \\
\hline & 4 & 94.69 & 14.40 \\
\cline { 2 - 4 } & 5 & 96.26 & 7.41 \\
\cline { 2 - 4 } & Overall & 11. \\
\hline
\end{tabular}


Table 32. Value of the evaluated dependent variables by semiotic density.

\begin{tabular}{l|l|r|r}
\hline Dependent variable & Semiotic density & Average & SD \\
\hline \multirow{3}{*}{ Exploration \& Curiosity time } & Low & 41.46 & 13.82 \\
\cline { 2 - 4 } & High & 46.72 & 12.50 \\
\cline { 2 - 4 } & Overall & 44.43 & 13. \\
\hline \multirow{3}{*}{ Problem-solving Reasoning time } & Low & 13.75 & 5.16 \\
\cline { 2 - 4 } & High & 14.21 & 4.38 \\
\cline { 2 - 4 } & Overall & 14.01 & 4.7 \\
\hline \multirow{3}{*}{ Total camervation time } & Low & 44.79 & 15.08 \\
\cline { 2 - 4 } & High & 39.07 & 14.42 \\
\cline { 2 - 4 } & Overall & 41.55 & 14. \\
\hline \multirow{3}{*}{$\%$ of available cameras } & Low & 7.64 & 3.39 \\
\cline { 2 - 4 } & High & 9.00 & 3.15 \\
\cline { 2 - 4 } & Overall & 8.41 & 3.3 \\
\hline \multirow{2}{*}{ of gestures fitted } & Low & 100 & 0 \\
\cline { 2 - 4 } & High & 90.70 & 15.13 \\
\cline { 2 - 4 } & Overall & 94.74 & 12. \\
\hline & Low & 90.34 & 18.47 \\
\cline { 2 - 4 } & High & 96.38 & 11. \\
\cline { 2 - 4 } & Overall & & 0 \\
\hline
\end{tabular}

Table 33. Value of the evaluated dependent variables by gender.

\begin{tabular}{l|l|r|r}
\hline Dependent variable & Gender & Average & \multicolumn{1}{l}{ SD } \\
\hline \multirow{3}{*}{ Exploration \& Curiosity time } & Male & 45.10 & 15.08 \\
\cline { 2 - 4 } & Female & 43.89 & 11.75 \\
\cline { 2 - 4 } & Overall & 44.43 & 13. \\
\hline \multirow{3}{*}{ Problem-solving Reasoning time } & Male & 14.93 & 5.43 \\
\cline { 2 - 4 } & Female & 13.28 & 3.94 \\
\cline { 2 - 4 } & Overall & 14.01 & 4.7 \\
\hline \multirow{3}{*}{ Observation time } & Male & 39.97 & 16.71 \\
\cline { 2 - 4 } & Female & 42.83 & 13.31 \\
\cline { 2 - 4 } & Overall & 41.55 & 14. \\
\hline \multirow{3}{*}{$\%$ of available cameras } & Male & 8.29 & 3.25 \\
\cline { 2 - 4 } & Female & 8.50 & 3.38 \\
\cline { 2 - 4 } & Overall & 8.41 & 3.3 \\
\hline \multirow{2}{*}{$\%$ of gestures fitted } & Male & 93.63 & 14.80 \\
\cline { 2 - 4 } & Female & 95.64 & 99.78 \\
\cline { 2 - 4 } & Overall & 94.74 & 12. \\
\hline & Male & 97.06 & 8.74 \\
\cline { 2 - 4 } & Female & 95.83 & 13.71 \\
\cline { 2 - 4 } & Overall & 96.38 & 11. \\
\hline
\end{tabular}





\section{Part IV}

\section{Closure}





\section{Chapter 13}

\section{Discussion}

The research hypothesis of this work, as defined in Chapter 1, states that "Usability and communicability strategies can be effectively designed to establish a basis for the development of applications based on touchscreen interfaces targeted to kindergarten children taking into account their development stage and abilities. This basis will provide an added value in terms of user experience, performance, engagement and quality of the educational activities and dialogues with teachers or caretakers". To prove this hypothesis, we have conducted a series of studies in which several usability and communicability strategies are proposed and evaluated pointing out the design guidelines to be used to adapt them to be used by kindergarten children. The results obtained in these studies lead us to accept the main research hypothesis defined for this work since several usability and communicability strategies have been designed, developed and successfully tested showing that they can be included in applications based on touchscreen devices targeted to kindergarten children.

This thesis starts by revisiting previous works using technology with children and motivates the proposed work by highlighting the benefits of using a direct manipulation style and the direct touching offered by touchscreen devices. Then, Chapter 3 identifies a trend of challenges or future works to be carried out in the future to help in the task of creating more standard multi-touch interactions. Hence, starting from this point, the thesis explores and evaluates several milestones (see Figure 1) in terms of usability and communicability that will allow researchers and applications designers to know better how to develop applications appropriate and tailored to kindergarten children's skills and development. The evaluation of these milestones has been carried out step by step in order to provide a comprehensive understanding of kindergarten children abilities when interacting with touchscreen devices. In the 
Kindertivity: Usability and Communicability Strategies for Interactive Surfaces and PreKindergarten Children

following we discuss the evaluated techniques from the point of view of interaction usability and communication mechanisms.

\subsection{On multi-touch technology usability by kindergarten children}

Multi-touch technology is being widely used when developing applications targeted to kindergarten children. However, the interactions used in commercial applications are very limited avoiding the full exploitation of the technology. Most of these applications use only two touch gestures (tap and drag) to interact with the virtual elements. This fact motivated the work carried out in Chapter 5 in order to assess whether kindergarten children are able to use a wider set of basic multi-touch gestures. The evaluation shows that the fundamental question "Are pre-kindergarten children ready for multi-touch technology?" is affirmatively answered since children aged 2 years and elders achieve similar levels of success for interactions such as one-finger rotation and two-finger scale up \& down, as for tap and drag, already mainstream in existing applications. This is an interesting point since it shows that the general belief on commercial applications designers that pre-school children are only capable of performing tap and drag interactions is really no more than a myth and the multi-touch technology can be more exploited providing more interaction points with several gestures to engage children in playful and educational tasks based on touchscreen devices.

Moreover, despite previous studies on children's development shows superior fine motor control in girls and that male toddlers' hands tend to be larger, no gender differences are reported in terms of success rate when performing these gestures. In terms of time, males needed, on average, less time than females when performing dynamic gestures (except the drag gestures) such as scale up, scale down and onefinger rotation. On the other hand, the results are in accordance to children cognitive development theories since they are in the process of developing their motor skills. Hence, despite there are no differences in terms of success rates when performing the gestures, the older ones performed faster in gestures such as double tap, drag, scale down and one-finger rotation. Therefore, designers should take into account these results when designing applications for children. If time is not a strong requirement of the application, the interactions can be the same but if the applications have time requirements it could be interesting to use an adaptive technique which fits the application requirements with the skills of each user depending on factors such as gesture, age or, eventually, gender.

In addition, the experimentation carried out in Chapter 5 reports performance issues with gestures requiring more advanced skills that children have not developed yet such as mathematical thinking, bi-manual coordination and spatial-geometric interpretation to plan a complex gesture in advance. In this respect, the two-finger rotation gesture results inappropriate to be used with very young children and should not be included in future applications. This fact is in line with other previous studies which have shown 
that this is also important for adult users (Nacenta et al., 2009), although not all manipulations might require this planning in advance, which makes it an even more important point for designers. From these observations, three design guidelines are proposed when developing applications for kindergarteners. The first one recommends avoiding the inclusion of interactions that involve mathematical concepts such as counting exact numbers or repeating a given number of events. Second, to avoid using interactions which require complex coordination processes involving both hands is advised. And thirdly, it is recommended to avoid interactions that require the effective estimation of spatial relationships if performance (in terms of time needed to perform the task) is a mandatory requirement.

Finally, the evaluation also shows that kindergarteners have some issues when performing gestures such as double tap and long pressed achieving lower success rates that may lead to prevent its use in future applications. However, several interaction aids or design guidelines are proposed in order to adapt these gestures to young children's development. In the case of the long press gesture, the supervisors observed through the experimentation that spurious dragging events in the initial phase of the gesture produced by the lack of precision of kindergarten children prevent the system from detecting the gesture despite that they hold their finger on the target. Hence, the need to design filtering processes for spurious undesired events that may occur in the acquisition/initial phase of any interaction is stated. On the other hand, in the double tap gesture, supervisors pointed out that the standard time required between taps predefined by the system is much shorter than the actual pace at which kindergarteners are able to perform two consecutive contacts due to their limited motor development. Hence, to design adaptive mechanisms to match the required speed for time-based interactions to the actual ability of the user is proposed when developing applications targeted to children. Therefore, the definition, implementation and evaluation of these two design guidelines is carried out in Chapter 6 in order to assess their efficacy. The results showed that including the proposed design guidelines makes the long pressed and double tap gestures feasible for kindergarten children since they reach success rates similar to the gestures successfully tested before (see Figure 86).

It should be taken into account that despite young children are able to perform a wide set of basic multi-touch gestures, they experience difficulties with certain movements that need more highly honed skills. This points out that special attention must be paid to the finalization phase of gestures which require high levels of precision in the termination phase of the action (such as drag, scale up, scale down and one-finger rotation). In order to find out the appropriate precision levels that can be expected from children when performing such gestures, Chapter 7 explores children's accuracy when high levels of precision are demanded. The evaluation points out that the age is a factor to be seriously taken into account when high levels of accuracy are demanded to children when performing touch gestures. Except the drag gesture, that is feasible for children since they are aged two years old, requiring precision in termination phase of more complex gestures such as rotation, scale up and scale down show has a high 
Kindertivity: Usability and Communicability Strategies for Interactive Surfaces and PreKindergarten Children

impact in terms of success and accuracy achieved on less than 30 months children. As it is known, children are in the pre-operational stage of their development and are improving its motor skills continuously (Piaget, 1973) so their performance presents high variability. In this line, the evaluation also shows that, on average, girls are more successful than boys when precision is required. This fact is consistent with previous work on children development which shows superior fine motor skills in girls (Moser \& Reikerås, 2014).

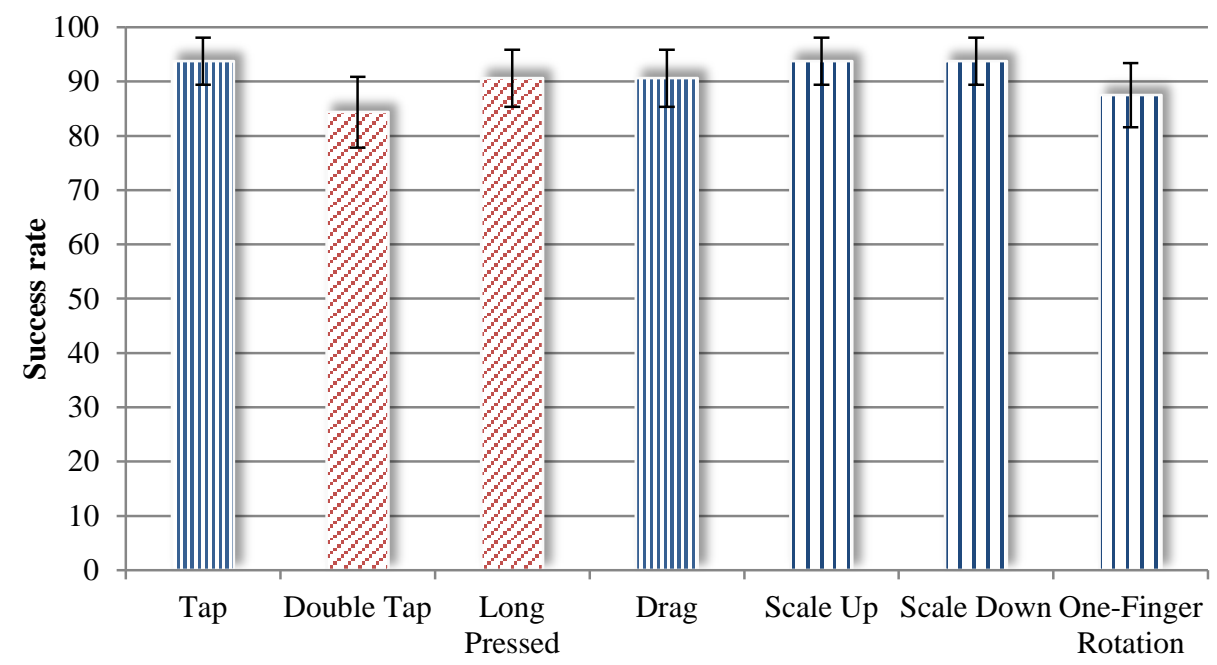

Figure 86. Kindergarteners' success rate achieved by gesture when applying design guidelines in the Double Tap and Long Pressed gestures.

Therefore, performing dynamic gestures with high accuracy is not always feasible and, as stated above, age is a key factor since kindergarten children are in continuous development and older children are able to achieve significant higher levels of accuracy than younger ones. Moreover, gender has also an impact since fine motor skills development is not equal in males and females. Hence, we propose the implementation of boundary detection components that detect when an object gets close to its final desired state. In this respect, we propose a design guideline in which boundary detection components are designed to cope with precision problems related to the exact final state of an interactive element. These components can automatically adapt their behavior and thresholds by using assistive strategies that adapt them to the actual levels of motor and cognitive development of each child. This would be in concordance with the principles of differentiated instruction (Subban, 2006) and, by using this guideline, designers will avoid the problem that arises when fixed thresholds are predefined relative to preventing the more skilled children to exercise and further enhance their precision related skills. 
Although previously we point out that kindergarten children are able to perform a set of basic multi-touch gestures, all the evaluations reported above explored the gestures in isolation. As can be expected, there will be several scenarios in which these gestures will be performed in sequences and, as have been reported in previous works, the effectiveness of performing some gestures can be reduced by previous and subsequent events when they are concatenated (Hinrichs \& Carpendale, 2011). In this respect, aiming to assess whether kindergarten children are able to perform sequences of different multi-touch gestures, Chapter 12 presents an application evaluation including several scenarios in which gestures such as long pressed, drag, scale up, scale down and rotation are performed consecutively. The experimentation shows that sequencing these touch gestures does not have any impact in performance in terms of time or success rate. This is an interesting result as it empowers application designers to develop applications requiring the use of different gestures in sequences.

Finally, Chapter 11 provides the experimental confirmation that kindergarteners are able to use the space beyond the screen limits as a virtual world (i.e. the application camera can be moved to reach other parts not displayed at a given time). This is an interesting result since most of commercial applications targeted to kindergarten children limit their virtual world to what is being displayed on the screen but this confirmation opens new opportunities to designers. For example, Golbeck (Golbeck, 2005) affirms that "with experience and growing memory capabilities, children shift from a uni-axial to a bi-axial system for spatial representation", hence, designing applications in which spatial awareness is exercised may help children make this shift and improve their spatial literacy. Moreover, developing applications which use the augmented space beyond the screen limits will help to define scenarios with tasks driven by curiosity and discovery which are identified as a suitable and desired way for children learning (Honomichl \& Chen, 2012) (Bruner, 1960) (Collins \& Stevens, 1981).

To sum up, from a usability point of view, the thesis explores and evaluates different interactions in order to assess their suitability to be included in touchscreen applications targeted to kindergarten children. This exploration provides a successful set of interactions feasible for children in their early development and points out the factors to be taken into account by designers to adapt their applications to the actual children's skills.

\subsection{On communicability strategies targeted to young children}

Once the thesis finds out which type of interactions are the most suitable for kindergarten children, it investigates the suitability of several visual languages to communicate information to users.

First, Chapter 9 presents a preliminary work to evaluate how to advise users of the gestures they are expected to perform in a multi-touch application. This work compares 
Kindertivity: Usability and Communicability Strategies for Interactive Surfaces and PreKindergarten Children

the suitability of using an iconic approach versus an animated language based on a moving hand. The results show that despite children have a poor performance when understanding in-place gestures (i.e. tap) in both approaches, children are able to understand the animated approach when communicating dynamic touch gestures (i.e. those that involve movement of contact points on the surface). This is a valuable finding because despite developmental psychologists such as Levine and Piaget suggest that kids develop spatial reasoning during middle childhood (Levine et al., 1999) (Piaget, 1973) this experiment suggests that the basic reasoning related to the interpretation of moving elements on a surface can be effectively performed during early childhood. Given these results, Chapter 10 makes a comprehensive exploration of visual languages with a wider set of gestures. The results reassert the claim that inplace gestures cannot be effectively communicated. Hence, we can conclude that the reasoning involving a process of classification and association of a visual stimulus to a gesture whose nature cannot be interpreted in terms of spatial analogy with the stimulus is a more complex type of reasoning and, as pointed out by Piaget, this capability is developed by children at a later stage. On the other hand, this paper reaffirms that animated visual approaches are effective to communicate dynamic gestures. In this sense, two approaches (one with the explicit representation of a hand and one avoiding it) are designed and evaluated revealing that the communication of the simplest gestures (such as drag and rotation) is easily learned after an initial immediate recall phase and children achieve high success rates to understand them. In the case of communicating gestures with two contact points, the animated approaches are not as intuitive as when communicating gestures with only one moving contact but the languages seem learnable by kindergarten children since their effectiveness is improved after a training process reaching success rates over $90 \%$. Therefore, using animated visual cues seems a suitable strategy to communicate the gestures to be performed to kindergarten children.

Secondly, Chapter 11 explores three different strategies to find out whether it is possible to communicate directional awareness to kindergarteners. The results show that the three techniques are understandable for children aged 4 years old and elder since they achieve success rates of over $90 \%$ in a task requiring directional information to be completed. These high success rates suggest that even very young children are able to perform the required mapping to interpret the data given by visual languages and extrapolate it to locate different objects in a virtual world. However, despite the high success rates achieved, the mini-map technique, usually used with adult users, resulted the most problematic, specially, for the youngest children. This is consistent with Piaget development statements (Piaget, 1973) which point out that children begin their preoperational stage at 24 months and then gradually develop layers of symbolic behaviors as they are introduced to reading symbols (Gardner, 1993), so the youngest children have some issues when understanding the information communicated by the mini-map technique. In addition, this work provides several advices and guidelines for designers to better choose the most appropriate visual language to propitiate an 
effective communication process depending on application's requirements in terms of success, time, visual interference, relative positioning awareness efficacy and user's age. These results and guidelines are a valuable contribution since they would enable the creation of educational scenarios in which the digital space to be explored exceeds the physical boundaries of the screen fostering the benefits of this type of tasks that have been pointed out above. Moreover, using these techniques would help to develop applications which support the development of spatial and directional skills which is an important cognitive ability at this early age.

Lastly, in terms of communicability, in Chapter 12 the possible impact of including simultaneously two visual languages in an application to communicate information from different contexts (i.e. gestures to be performed and directional awareness) is explored. The results showed that despite being used simultaneously, visual languages efficacy is not affected and children are able to understand the information communicated with high proficiency. Moreover, this chapter reports a preliminary study of a real application showing that the usage of visual languages to communicate technical information about the task to be performed reduces the technical interferences in dialogues helping supervisors to foster richer conversations around the educational content. These results open new opportunities to designers to develop applications using several communication languages at a time since children aged 4 and older are able to understand and process this information without having an impact in the performance of the task.

To sum up, kindergarten children are in the process of early language development and the younger the children are the more scaffolding of technical nature they need (Neumann, 2017), including special communication strategies when using touch screen devices. Hence, the effectiveness of the provided visual languages to communicate different application information will avoid the continuous technical scaffolding by adults (i.e. the gestures to be performed, the direction in which a game character should move, etc.) and will help caretakers to concentrate on giving cognitive scaffolding (i.e. the learning content to be acquired by the children). This will lead to develop applications that trigger and enhance collaborative dialogic learning with the participation of educators or supervisors.

Finally, despite this work explores a set of strategies feasible and suitable to be used when designing applications targeted to kindergarten children based on touchscreen devices, it is worth noting that the American Academy of Pediatrics (AAP Council on communications and media, 2016) recommends avoiding the use of touch screen devices for children younger than 18 months and they also recommend a responsible usage with a limit of one hour of screen per day, with parents co-viewing and collaborating with the children's interactions when they are between two and five years old. These recommendations about time usage and screen devices exposition should seriously be taken into account by educators, caretakers and parents when using these 
Kindertivity: Usability and Communicability Strategies for Interactive Surfaces and PreKindergarten Children

devices in both, educational and playful scenarios. It is also important to highlight that the use of touch technology cannot replace essential activities needed by young developing children, such as jumping, running, interacting with others, learning social interaction norms, etc. 


\section{Chapter 14}

\section{Conclusions and Future Work}

This thesis has contributed to the study and definition of several techniques and strategies to be used when developing applications targeted to children in their early development based on multi-touch technology devices. Throughout this manuscript, it has been exposed that the use of multi-touch technology with kindergarten children is feasible, suitable and engaging for them. This work establishes the basis to design and develop applications targeted to kindergarteners by defining several strategies and guidelines in terms of usability and communicability according to children's actual development and abilities. This manuscript addresses the definition and evaluation of these strategies throughout the different chapters and the most relevant are highlighted below.

The thesis starts with a thorough exploration of the literature related to the use of interactive technologies aiming to identify the strengths and possibilities that emergent technologies provide. This exploration of the literature begins with a broad view (i.e. including robots, tangible-user interfaces, etc.) to finish with a specific review of the possibilities offered by multi-touch technologies and touchscreen devices to support applications targeted to kindergarten children.

Once the suitability of multi-touch technology is pointed out, the thesis addresses several works aiming to contextualize which touchscreen interactions are feasible to be used with young users. In this respect, this work provides, first, a review from the commercial perspective with the purpose of finding out which interactions are used by commercial designers to develop applications targeted to kindergarten children. Several 
Kindertivity: Usability and Communicability Strategies for Interactive Surfaces and PreKindergarten Children

conclusions arise from the study; the first one is that there is a clear trend to develop playful and educational applications targeted to children and, second, that these applications are not fully exploiting the multi-touch technology potential since the interactions supported are limited to basic gestures such as tap and drag limiting the interactivity of the virtual elements of the application.

Therefore, aiming to define appropriate design strategies and guidelines that enable infants to take full advantage of multi-touch technology, the thesis addresses the exploration and evaluation of different types of touch interactions feasible to be performed by kindergarten children in multi-touch surfaces. In this respect, a basic set of touch gestures (such as tap, drag, scale up, scale down and rotation) suitable to kindergarten interaction is provided. Moreover, the thesis provides a successful set of design guidelines which will help to avoid precision and cognitive limitations of young children in order to include more complex touch gestures (such as double tap and long pressed) in future applications. On the other hand, the thesis explores and finds out that despite the early motor and cognitive development of kindergarteners, they are able to successfully perform gestures that require movement of contacts in the surface when accuracy is required in the termination phase of the action. This will lead to the design of future multi-touch application using assistive strategies that adapt their behavior to the actual levels of motor and cognitive development of each child supporting children exercising allowing them to improve precision motor related skills at an early phase of their development. Besides, in terms of usability, the thesis provides several indirect drag techniques to be used in scenarios in which direct dragging is not appropriate (i.e. when the object to be dragged has to move at a fixed speed or performing an animation independent of the actual direct movement speed or trajectory of the user's hand). Three indirect techniques are designed and evaluated resulting all of them feasible for children aged 4 or elder. However, depending on the application's requirements on several factors (such as the effectiveness, efficiency or precision required), a set of guidelines to use the appropriate technique for each scenario is defined and detailed. Finally, the thesis also states that even though commercial applications do not use the space beyond the screen limits as a virtual world, kindergarten children are able to successfully use this augmented space.

In terms of communicability, the thesis states the need for appropriate communication mechanisms in order to reduce the technical scaffolding (e.g. information about the interactions expected by the application or about where is expected to be moved the main character) provided by parents or caretakers when using touchscreen devices. Then, designing visual languages understandable for kindergarten children will foster richer dialogues between children and supervisors focusing on the learning or playful content instead of continuously giving instructions about how to interact or how to proceed to achieve the game goals. In this respect, the thesis proposes visual languages to communicate information from different scopes. First, the thesis explores different visual languages to communicate the gestures to be performed by children to fulfill a given task in a touchscreen device. The thesis provides two animated visual languages 
and its evaluation revealed that both are a successful way to communicate gestures which involve movements of contacts on the surface such as Drag, Scale up, Scale down and Rotation. However, the results revealed that using these visual languages to communicate in-place interactions (those in which one hand does not actually describe a trajectory but taps at a specific or in a specific way) is not intuitive and learnable by young children. In addition, the experimentation carried out shows that using static iconic visual cues as the ones usually employed with adult users is not appropriate for children in their early development since the process of classification and association of a visual stimulus to a gesture is developed in a later stage of the children's cognitive development. Secondly, the thesis explores different ways to communicate directional awareness information to kindergarten children. The evaluation carried out shows that the mini-map approach usually used with adults was the most problematic one, whereas the two other proposed techniques (border-floating thumbnails and arrow based) reached success rates of over $99 \%$ being suitable and understandable by kindergarten children despite their early development. In addition, several guidelines are defined in terms of time, relative positioning awareness and degree of interference of the visual language to be taken into account by designers when developing applications in order to choose the most appropriate technique to fit the applications requirements.

Finally, the thesis provides an adaptation of an actual application targeted to children aged between 8 and 18 years by gamifying it to kindergarteners' development and skills using most of the strategies and guidelines proposed and successfully evaluated through this work. The results show that kindergarten children are able to use it and by using this game dialogues with caretakers about the learning content to be acquired are fostered.

This thesis opens several questions that should be addressed in the future. An interesting issue to address in the future could be the definition of strategies that allow the adaptation of the usability and communicability guidelines proposed in this work to the actual levels of motor and cognitive development of each user. This adaptation will help to develop applications appropriate to children with different levels of skills development avoiding rigid and predefined designs which could prevent the more skilled children from exercising and further enhancing their precision related skills at an early phase of their development.

Another of our plans for future work includes the design and evaluation of an educational scenario to study the impact of including the proposed visual languages in instructors' or parents' dialogic strategies during the learning process. The results will show whether using these visual languages can improve the learning process by helping parents to focus their dialogues on the learning content rather than on other technical instructions.

Regarding the communication strategies targeted to inform about directional and spatial information, it will be worth to study whether the results of the most successful 
Kindertivity: Usability and Communicability Strategies for Interactive Surfaces and PreKindergarten Children

visual languages proposed in this work can be extrapolated to different situations in which directional awareness is developed and communicated in a 3D space explored by kindergarteners with the use of digitally augmented tricycles (Tanaka \& Takahashi, 2012) or collaborative robots (Garcia-Sanjuan, Jaen, Nacher, et al., 2015).

Finally, once known the actual capabilities and abilities of kindergarten children, in terms of usability (i.e. the gestures that they can perform, the accuracy that they can achieve) and evaluated different communicative strategies suitable for their abilities and skills; an interesting strand of future work would be to define, construct and evaluate educational scenarios based on touchscreen devices that foster creativity and allow collaboration between peers and teachers. A starting point to develop these environments could be taking advance of two characteristics of tablets and smartphones; (1) the portability of tablets that allow children and teachers to use various locations in the classroom enabling the creativity and collaboration of small groups of children; and (2) the more sophisticated interface of tablets that allows both individual and mutual interaction between two or more users (Wakefield \& Smith, 2012). The use of the strategies in terms of usability and communicability defined and tested in this thesis will facilitate the integration of these environments into actual classrooms to be used with educational purposes allowing children to develop their creativity skills and allow educators to have richer dialogues with children focused in the leaning content, to monitor the progress of their students and to create appropriate content tailored for them. The creation of this type of classroom environments that enhance students' expression and selection opportunities will provide a proper atmosphere towards helping children to learn at their own pace (Rushton \& JuolaRushton, 2008). 


\section{Bibliography}

AAP Council on communications and media. (2016). Media and Young Minds. Pediatrics, 138(5), e20162591. https://doi.org/10.1542/peds.2016-2591

Abdi, H. (2010). The greenhouse-geisser correction. Encyclopedia of Research Design. Sage Publications, 544-548. https://doi.org/10.1007/BF02289823

Abdul Aziz, N. A., Batmaz, F., Stone, R., \& Paul, C. (2013). Selection of touch gestures for children's applications. In Proceedings of the Science and Information Conference (pp. 721-726). London, UK.

Abdul Aziz, N. A., Mat, N. S., Batmaz, F., Stone, R., \& Paul, C. (2014). Selection of Touch Gestures for Children's Applications: Repeated Experiment to Increase Reliability. International Journal of Advanced Computer Science and Applications, 5(4), 97-102.

Ahlström, D., \& Hitz, M. (2013). Revisiting PointAssist and Studying Effects of ControlDisplay Gain on Pointing Performance by Four-Year-Olds. In Proceedings of the 12th International Conference on Interaction Design and Children (pp. 257-260). ACM. https://doi.org/10.1145/2485760.2485792

Allen, R., \& Scofield, J. (2010). Word learning from videos: more evidence from 2-yearolds. Infant and Child Development, 19(6), 649-661. https://doi.org/10.1002/icd.712

American Academy of Pediatrics. (2011). Media Use by Children Younger Than 2 Years. Pediatrics, 128(5), 1040-1045. https://doi.org/10.1542/peds.2011-1753

Anderson, D. R., Huston, A. C., Schmitt, K. L., Linebarger, D. L., \& Wright, J. C. (2001). Early childhood television viewing and adolescent behavior: The Recontact Study. Monographs of the Society for Research in Child Development, 66(1), 1-147. https://doi.org/10.1111/1540-5834.00120

Apple. (2013). iOS. Retrieved July 16, 2013, from http://www.apple.com/es/ios/ios7/?cid=wwa-es-kwg-iphone

Arce, E. (2013). Curriculum for young children.

Baloian, N., Pino, J. a., \& Vargas, R. (2013). Tablet gestures as a motivating factor for 
Kindertivity: Usability and Communicability Strategies for Interactive Surfaces and PreKindergarten Children

learning. Proceedings of the 2013 Chilean Conference on Human - Computer Interaction - ChileCHI '13, 98-103. https://doi.org/10.1145/2535597.2535622

Barnett, L. (1990). Developmental benefits of play for children. Journal of Leisure Research, 22(2), 138-153.

Basili, V. R., Caldiera, G., \& Rombach, H. D. (1994). The Goal Question Metric Approach. In Encyclopedia of Software Engineering (Vol. 2, pp. 528-532). Wiley. https://doi.org/123

Bebell, D., Dorris, S., \& Muir, M. (2012). Emerging Results From The Nation's First Kindergarten Implementation of iPads. Auburn. Retrieved from https://s3.amazonaws.com/hackedu/Adv2014_ResearchSum120216.pdf

Berggren, J., \& Hedler, C. (2014). CamQuest: Design and Evaluation of a Tablet Application for Educational Use in Preschools. In Proceedings of the 2014 conference on Interaction design and children - IDC '14 (pp. 185-188). https://doi.org/10.1145/2593968.2610448

Berglund, E., Eriksson, M., \& Westerlund, M. (2005). Communicative skills in relation to gender, birth order, childcare and socioeconomic status in 18-month-old children. Scandinavian Journal of Psychology, 46(6), 485-491.

Bobick, A. F., Intille, S. S., Davis, J. W., Baird, F., Pinhanez, C. S., Campbell, L. W., ... Wilson, A. (1999). The KidsRoom: A Perceptually-Based Interactive and Immersive Story Environment. Presence: Teleoperators and Virtual Environments, 8(4), 369393. https://doi.org/10.1162/105474699566297

Bruner, J. S. (1960). The Act of Discovery. Philosophy of Education, 137.

Burton, L. A., Henninger, D., \& Hafetz, J. (2005). Gender differences in relations of mental rotation, verbal fluency, and SAT scores to finger length ratios as hormonal indexes. Developmental Neuropsychology, 28, 493-505. Retrieved from http://www.ncbi.nlm.nih.gov/pubmed/15992253

Buxton, B. (2013). Multi-touch systems that I have known and loved. Retrieved from http://billbuxton.com/multitouchOverview.html

Case, R., Griffin, S., \& Kelly, W. (2001). Socio-economic differences in children's early cognitive development and their readiness for schooling. Psychological Perspectives on Early Childhood Education: Reframing Dilemmas in Research and Practice, 3663. 
Case, R., Stephenson, K. M., Bleiker, C., \& Okamoto, Y. (2008). V. CENTRAL SPATIAL STRUCTURES AND THEIR DEVELOPMENT. Monographs of the Society for Research in Child Development, 61(1-2), 103-130. https://doi.org/10.1111/j.15405834.1996.tb00539.x

Catala, A., Jaen, J., van Dijk, B., \& Jordà, S. (2012). Exploring tabletops as an effective tool to foster creativity traits. In 6th International Conference on Tangible, Embedded and Embodied Interaction (TEI'12) (pp. 143-150). New York, New York, USA: ACM Press. https://doi.org/10.1145/2148131.2148163

Catling, S. (2005). Developing children's understanding and use of maps. In C. C. (eds) Lee, C. and Hung (Ed.), Primary Social Studies: Exploring Pedagogy and Content. Singapore: Marshall Cavendish Education.

Chen, a., Muntz, R. R., Yuen, S., Locher, I., Sung, S. I., \& Srivastava, M. B. (2002). A support infrastructure for the smart kindergarten. IEEE Pervasive Computing, 1(2), 49-57. https://doi.org/10.1109/MPRV.2002.1012337

Chiong, C., \& Shuler, C. (2010). Learning: Is there an app for that? Investigations of young children's usage and learning with mobile devices and apps. New York.

Collins, A., \& Stevens, A. L. (1981). Goals and Strategies of Inquiry Teachers (Advances i). Hillsdale, N.J.: Lawrence Erlbaum Associates.

Comisión Europea. (2009). Conclusiones del Consejo de 12 de mayo de 2009 sobre un marco estratégico para la cooperación europea en el ámbito de la educación y la formación ( 2 ET 2020»). Retrieved from http://eurlex.europa.eu/LexUriServ/LexUriServ.do?uri=OJ:C:2009:119:0002:0010:ES:PDF

Common Sense Media. (2013). Zero to Eight: Childrens Media Use in America 2013.

Cook, D. J., Augusto, J. C., \& Jakkula, V. R. (2009). Ambient intelligence: Technologies, applications, and opportunities. Pervasive and Mobile Computing, 5(4), 277-298. https://doi.org/10.1016/j.pmcj.2009.04.001

Couse, L. J., \& Chen, D. W. (2010). A Tablet Computer for Young Children? Exploring Its Viability for Early Childhood Education. Journal of Research on Technology in Education, 43(1), 75-98.

Cristia, A., \& Seidl, A. (2015). Parental Reports on Touch Screen Use in Early Childhood. PLOS ONE, 10(6), e0128338. https://doi.org/10.1371/journal.pone.0128338

Cropley, A. J. (2001). Creativity in Education and Learning: A Guide for Teachers and 
Kindertivity: Usability and Communicability Strategies for Interactive Surfaces and PreKindergarten Children

Educators. Kogan Page.

Damon, W., Lerner, R. M., Kuhn, D., \& Siegler, R. S. (Eds.). (2006). Handbook of Child Psychology, Volume 2, Cognition, Perception, and Language (6th ed.). Wiley.

Demetriou, A., Christou, C., Spanoudis, G., \& Platsidou, M. (2002). The Development of Mental Processing: Efficiency, Working Memory, and Thinking. Monographs of the Society for Research in Child Development, 67(1), 1-167. https://doi.org/10.2307/3181583

Dempsey, J. V., Rasmussen, K., \& Lucassen, B. (1994). Instructional gaming: Implications for instructional technology. Annual Meeting of the Association for Educational Communications and Technology, 1-21.

Derboven, J., De Roeck, D., \& Verstraete, M. (2012). Semiotic analysis of multi-touch interface design: The MuTable case study. International Journal of Human-Computer Studies, 70(10), 714-728. https://doi.org/10.1016/j.ijhcs.2012.05.005

Donker, A., \& Reitsma, P. (2007). Drag-and-drop errors in young children's use of the mouse. Interacting with Computers, 19(2), 257-266. https://doi.org/10.1016/j.intcom.2006.05.008

Downs, R. M., Liben, L. S., \& Daggs, D. G. (1988). On Education and Geographers: The Role of Cognitive Developmental Theory in Geographic Education. Annals of the Association of American Geographers, 78(4), 680-700. https://doi.org/10.1111/j.1467-8306.1988.tb00238.x

Egloff, T. H. (2004). Edutainment: a case study of interactive cd-rom playsets. Computers in Entertainment, 2(1), 13. https://doi.org/10.1145/973801.973822

Falloon, G. (2013). Young students using iPads: App design and content influences on their learning pathways. Computers and Education, 68, 505-521. https://doi.org/10.1016/j.compedu.2013.06.006

Fein, G. G. (1981). Reviews Pretend Play in Childhood: An Integrative Review. Child Development, 52(4), 1095-1118. https://doi.org/10.1111/1467-8624.ep8860387

Fender, J. G., Richert, R. A., Robb, M. B., \& Wartella, E. (2010). Parent teaching focus and toddlers' learning from an infant DVD. Infant and Child Development, 19(6), 613627. https://doi.org/10.1002/icd.713

Fernández-López, Á., Rodríguez-Fórtiz, M. J., Rodríguez-Almendros, M. L., \& MartínezSegura, M. J. (2013). Mobile learning technology based on iOS devices to support 
students with special education needs. Computers \& Education, 61, 77-90. https://doi.org/10.1016/j.compedu.2012.09.014

Fielding-barnsley, R. (2002). Developing Pre-Literacy Skills via Shared Book Reading. Australian Journal of Learning Disabilities, 7(3), 13-19.

Fleck, R., Rogers, Y., Yuill, N., Marshall, P., Carr, A., Rick, J., \& Bonnett, V. (2009). Actions speak loudly with words. In ACM International Conference on Interactive Tabletops and Surfaces (ITS'09) (pp. 189-196). New York, New York, USA: ACM Press. https://doi.org/10.1145/1731903.1731939

Frost, J. L., Wortham, S. C., \& Reifel, S. (2011). Play and Child Development. Pearson. Retrieved from https://books.google.es/books?id=OLE2YgEACAAJ

Furió, D., González-Gancedo, S., Juan, M. C., Seguí, I., \& Rando, N. (2013). Evaluation of learning outcomes using an educational iPhone game vs. traditional game. Computers and Education, 64, 1-23. https://doi.org/10.1016/j.compedu.2012.12.001

García, N. M. F., Vela, F. L. G., Rodríguez, P. P., Arcos, R. L., \& Zea, N. P. (2015). Teaching Emotions to Children by Using Video Games. Proceedings of the XVI International Conference on Human Computer Interaction - Interacción '15, 1-3. https://doi.org/10.1145/2829875.2829888

Garcia-Sanjuan, F., Jaen, J., Catala, A., \& Fitzpatrick, G. (2015). Airsteroids: Re-designing the Arcade Game Using MarkAirs. In Proceedings of the 2015 International Conference on Interactive Tabletops \& Surfaces - ITS '15 (pp. 413-416). New York, New York, USA: ACM Press. https://doi.org/10.1145/2817721.2823480

Garcia-Sanjuan, F., Jaen, J., Nacher, V., \& Catala, A. (2015). Design and Evaluation of a Tangible - Mediated Robot for Kindergarten Instruction. In 12th International Conference on Advances in Computer Entertainment Technology. Iskandar, Malaysia. https://doi.org/10.1145/2832932.2832952

Gardner, H. (1993). The Unschooled Mind. London, UK: Fontana Press.

Ghosh, M., \& Tanaka, F. (2011). The impact of different competence levels of carereceiving robot on children. In IEEE International Conference on Intelligent Robots and Systems (pp. 2409-2415). IEEE. https://doi.org/10.1109/IROS.2011.6048743

Ginsburg, K. R. (2007). The Importance of Play in Promoting Healthy Child Development and Maintaining Strong Parent-Child Bonds. PEDIATRICS, 119(1), 182-191. https://doi.org/10.1542/peds.2006-2697 
Kindertivity: Usability and Communicability Strategies for Interactive Surfaces and PreKindergarten Children

Golbeck, S. L. (2005). Building Foundations for Spatial Literacy in Early Childhood. $\begin{array}{llll}\text { Proquest } \quad \text { Journals, } & \text { 60(6), } & \text { Retrieved from }\end{array}$ http://www.pdcbank.state.nj.us/education/ece/pd/spatial/BuildingFoundationforSpatia lLiteracyinEarlyChildhood.pdf

González, P., Montero, F., López, V., Fernández-Caballero, A., Montañés, J., \& Sánchez, T. (2001). A Virtual Learning Environment for Short Age Children. In IEEE International Conference on Advanced Learning Technologies (ICALT'01) (pp. 283284). IEEE.

González-González, C. S., \& Navarro-Adelantado, V. (2015). Methods and techniques for evaluating the emotional experiences of children with active videogames. In Proceedings of the XVI International Conference on Human Computer Interaction Interacción '15 (pp. 1-2). New York, New York, USA: ACM Press. https://doi.org/10.1145/2829875.2829883

Google. (2013). Android. Retrieved July 16, 2013, from http://www.android.com/

Goria, S., \& Papadopoulou, M. (2012). Icons versus symbols: investigating preschoolers' cartographic design, 5, 1-18.

Han, J., Jo, M., Park, S., \& Kim, S. (2005). The educational use of home robots for children. In ROMAN 2005. IEEE International Workshop on Robot and Human Interactive Communication, 2005. (pp. 378-383). IEEE. https://doi.org/10.1109/ROMAN.2005.1513808

Hanna, L., Risden, K., \& Alexander, K. (1997). Guidelines for usability testing with children. Interactions, 4(5), 9-14. https://doi.org/10.1145/264044.264045

Harris, A., Rick, J., Bonnett, V., Yuill, N., Fleck, R., Marshall, P., \& Rogers, Y. (2009). Around the table: are multiple-touch surfaces better than single-touch for children's collaborative interactions? In 9th International Conference on Computer Supported Collaborative Learning (CSCL'09) (pp. 335-344). International Society of the Learning Sciences.

Helmes, J., Cao, X., Lindley, S. E., \& Sellen, A. (2009). Developing the story: Designing an Interactive Storytelling Application. In ACM International Conference on Interactive Tabletops and Surfaces (ITS'09) (pp. 49-52). New York, New York, USA: ACM Press. https://doi.org/10.1145/1731903.1731913

Hevner, March, Park, \& Ram. (2004). Design Science in Information Systems Research. MIS Quarterly, 28(1), 75. https://doi.org/10.2307/25148625 
Hiniker, A., Sobel, K., Hong, S. R., Suh, H., Irish, I., Kim, D., \& Kientz, J. a. (2015). Touchscreen Prompts for Preschoolers: Designing Developmentally Appropriate Techniques for Teaching Young Children to Perform Gestures. Proceedings of the 14th International Conference on Interaction Design and Children, 109-118. https://doi.org/10.1145/2771839.2771851

Hinrichs, U., \& Carpendale, S. (2011). Gestures in the wild: studying multi-touch gesture sequences on interactive tabletop exhibits. In Annual Conference on Human factors in computing systems (CHI'11) (pp. 3023-3032). New York, New York, USA: ACM Press. https://doi.org/10.1145/1978942.1979391

Hirsh-Pasek, K., Zosh, J. M., Golinkoff, R. M., Gray, J. H., Robb, M. B., \& Kaufman, J. (2015). Putting Education in "Educational" Apps: Lessons From the Science of Learning. Psychological Science in the Public Interest, 16(1), 3-34. https://doi.org/10.1177/1529100615569721

Hofmeester, K., \& Wolfe, J. (2012). Self-revealing gestures: teaching new touch interactions in windows 8. In Proceedings of the 2012 ACM annual conference extended abstracts on Human Factors in Computing Systems Extended Abstracts CHI EA '12 (pp. 815-828). New York, New York, USA: ACM Press. https://doi.org/10.1145/2212776.2212855

Hoggan, E., Nacenta, M., Kristensson, P. O., Williamson, J., Oulasvirta, A., \& Lehtiö, A. (2013). Multi-Touch Pinch Gestures: Performance and Ergonomics. In ACM Interactive Tabletops and Surfaces (pp. 219-222). St. Andrews: ACM. https://doi.org/http://doi.acm.org/10.1145/2512349.2512817

Hoggan, E., Williamson, J., Oulasvirta, A., Nacenta, M., Kristensson, P. O., \& Lehtiö, A. (2013). Multi-touch rotation gestures: performance and ergonomics. In SIGCHI Conference on Human Factors in Computing Systems (CHI '13) (pp. 3047-3050). New York, New York, USA: ACM Press. https://doi.org/10.1145/2470654.2481423

Honomichl, R. D., \& Chen, Z. (2012). The role of guidance in children's discovery learning. Wiley Interdisciplinary Reviews: Cognitive Science, 3(6), 615-622. https://doi.org/10.1002/wcs.1199

Hourcade, J. P. (2007). Interaction Design and Children. Foundations and Trends ${ }^{\circledR}$ in Human-Computer Interaction, 1(4), 277-392. https://doi.org/10.1561/1100000006

Hourcade, J. P., Bullock-Rest, N. E., \& Hansen, T. E. (2011). Multitouch tablet applications and activities to enhance the social skills of children with autism spectrum disorders. 
Kindertivity: Usability and Communicability Strategies for Interactive Surfaces and PreKindergarten Children

Personal and Ubiquitous Computing, 16(2), 157-168.

https://doi.org/10.1007/s00779-011-0383-3

Huber, B., Tarasuik, J., Antoniou, M. N., Garrett, C., Bowe, S. J., \& Kaufman, J. (2016). Young children's transfer of learning from a touchscreen device. Computers in Human Behavior, 56, 56-64. https://doi.org/10.1016/j.chb.2015.11.010

Huebner, C. E. (2000). Promoting Toddlers' Language Development Through CommunityBased Intervention. Journal of Applied Developmental Psychology, 21(5), 513-535. https://doi.org/10.1016/S0193-3973(00)00052-6

Huizinga, J. (1985). Homoludens. Groningen: Wolters-Noordhoff.

Hwang, I., Jang, H., Park, T., Choi, A., Lee, Y., Hwang, C., ... Song, J. (2012). Leveraging children's behavioral distribution and singularities in new interactive environments: study in kindergarten field trips. Pervasive'12 Proceedings of the 10th International Conference on Pervasive Computing, 7319, 39-56. https://doi.org/10.1007/978-3642-31205-2

Ingram, A., Wang, X., \& Ribarsky, W. (2012). Towards the establishment of a framework for intuitive multi-touch interaction design. In International Working Conference on Advanced Visual Interfaces (AVI'12) (pp. 66-73). New York, New York, USA: ACM Press. https://doi.org/10.1145/2254556.2254571

Inkpen, K., Booth, K. S., Klawe, M., \& Upitis, R. (1995). Playing together beats playing apart, especially for girls. In The first international conference on Computer support for collaborative learning - CSCL '95 (pp. 177-181). Morristown, NJ, USA: Association for Computational Linguistics. https://doi.org/10.3115/222020.222164

Ioannou, A., Zaphiris, P., Loizides, F., \& Vasiliou, C. (2013). Let'S Talk About Technology for Peace: A Systematic Assessment of Problem-Based Group Collaboration Around an Interactive Tabletop. Interacting with Computers, iwt061-. https://doi.org/10.1093/iwc/iwt061

IST Advisory Group. (2001). Scenarios for ambient intelligence in 2010.

Jefatura del Estado. Ley Orgánica 2/2006, de 3 de mayo, de Educación. (2006). Retrieved from http://www.boe.es/boe/dias/2006/05/04/pdfs/A17158-17207.pdf

Johnson, L., Adams, S., \& Cummins, M. (2012). The NMC Horizon Report: 2012 K-12. Austin, Texas: The New Media Consortium.

Jokisch, M., Bartoschek, T., \& Schwering, A. (2011). Usability testing of the interaction of 
novices with a multi-touch-table in semi public space. In 14th international conference on Human-computer interaction: interaction techniques and environments (HCII'11) (pp. 71-80). Springer.

Jones, M., \& Liu, M. (1997). Introducing Interactive Multimedia to Young Children: A Case Study of How Two-Year-Olds Interact with the Technology. Journal of Computing in Childhood Education, 8(4), 313-343.

Kähkönen, M., \& Ovaska, S. (2006). Initial observations on children and online instructions. Proceeding of the 2006 Conference on Interaction Design and Children - IDC '06, 93-96. https://doi.org/10.1145/1139073.1139098

Kammer, D., Dang, R., Steinhauf, J., \& Groh, R. (2014). Investigating interaction with tabletops in kindergarten environments. In Proceedings of the 2014 conference on Interaction design and children - IDC '14 (pp. 57-66). New York, New York, USA: ACM Press. https://doi.org/10.1145/2593968.2593975

Keenan, T., Ruffman, T., \& Olson, D. R. (1994). When do children begin to understand logical inference as a source of knowledge? Cognitive Development, 9(3), 331-353. https://doi.org/10.1016/0885-2014(94)90010-8

Khandelwal, M., \& Mazalek, A. (2007). Teaching table: a tangible mentor for pre-k math education. In 1st International Conference on Tangible and Embedded Interaction (TEI'07) (pp. 191-194). New York, New York, USA: ACM Press. https://doi.org/10.1145/1226969.1227009

Kharrufa, A., Leat, D., \& Olivier, P. (2010). Digital mysteries: designing for learning at the tabletop. In ACM International Conference on Interactive Tabletops and Surfaces (ITS'10) (pp. 197-206). New York, New York, USA: ACM Press. https://doi.org/10.1145/1936652.1936689

Knoche, H., Rasmussen, N. A., \& Boldreel, K. (2014). Do Interactions Speak Louder than Words? Dialogic Reading of an Interactive Tablet-based E-book with Children between 16 Months and Three Years of Age. Interaction Design and Children, 285288. https://doi.org/10.1145/2593968.2610473

Kremer, K. E. (2012). Conducting Game User Experience Research with Preschoolers. In Workshop on Games User Research: practice, methods, and applications (collocated to CHI'12). ACM. Retrieved from http://hcigames.businessandit.uoit.ca/chigur/wpcontent/uploads/2012/04/gurchi2012_submission_7.pdf

Laird, D. (1985). Approaches To Training And Development. Addison-Wesley, Reading, 
Kindertivity: Usability and Communicability Strategies for Interactive Surfaces and PreKindergarten Children

Mass.

Lee, R. (n.d.). Gestures. Retrieved June 20, 2014, from http://gesturecons.com/

Leekam, S., Perner, J., Healey, L., \& Sewell, C. (2008). False signs and the non-specificity of theory of mind: Evidence that preschoolers have general difficulties in understanding representations. British Journal of Developmental Psychology, 26(4), 485-497. https://doi.org/10.1348/026151007X260154

Lever, R., \& Sénéchal, M. (2011). Discussing stories: On how a dialogic reading intervention improves kindergartners' oral narrative construction. Journal of Experimental Child Psychology, 108(1), 1-24. https://doi.org/10.1016/j.jecp.2010.07.002

Levine, S. C., Huttenlocher, J., Taylor, A., \& Langrock, A. (1999). Early sex differences in spatial skill. Developmental Psychology, 35(4), 940-949. https://doi.org/10.1037/0012-1649.35.4.940

Limperos, A. M., Buckner, M. M., Kaufmann, R., \& Frisby, B. N. (2015). Online teaching and technological affordances: An experimental investigation into the impact of modality and clarity on perceived and actual learning. Computers \& Education. https://doi.org/10.1016/j.compedu.2014.12.015

Linebarger, D. L. (2005). Infants' and Toddlers' Television Viewing and Language Outcomes. American Behavioral Scientist, 48(5), 624-645. https://doi.org/10.1177/0002764204271505

Louv, R. (2008). Last Child in the Woods: Saving Our Children From Nature-Deficit Disorder (Workman Pu). New York: Algonquin Books of Chapel Hill.

Malizia, A., \& Bellucci, A. (2012). The artificiality of natural user interfaces. Communications of the ACM, 55(3), 36. https://doi.org/10.1145/2093548.2093563

Malone, T. (1981). Toward a theory of intrinsically motivating instruction. Cognitive Science, 5(4), 333-369. https://doi.org/10.1016/S0364-0213(81)80017-1

Mansor, E. I., De Angeli, A., \& De Bruijn, O. (2008). Little fingers on the tabletop: A usability evaluation in the kindergarten. In 3rd IEEE International Workshop on Horizontal Interactive Human Computer Systems (pp. 93-96). IEEE. https://doi.org/10.1109/TABLETOP.2008.4660190

Mansor, E. I., De Angeli, A., \& de Bruijn, O. (2009). The fantasy table. In 8th International Conference on Interaction Design and Children (IDC'09) (pp. 70-79). New York, 
New York, USA: ACM Press. https://doi.org/10.1145/1551788.1551801

Marco, J., Cerezo, E., Baldasarri, S., Mazzone, E., \& Read, J. C. (2009). User-oriented design and tangible interaction for kindergarten children. In Proceedings of the 8th International Conference on Interaction Design and Children - IDC '09 (p. 190). New York, New York, USA: ACM Press. https://doi.org/10.1145/1551788.1551825

Marco, J., Cerezo, E., \& Baldassarri, S. (2009). Evaluating a Tangible Game Video Console for Kids. In 12th IFIP TC 13 International Conference Human-Computer Interaction (INTERACT 2009) (pp. 141-144). Springer. https://doi.org/10.1007/9783-642-03655-2_17

Markopoulos, P., \& Bekker, M. (2003). Interaction design and children. Interacting with Computers, 15(2 SPEC.), 141-149. https://doi.org/10.1016/S0953-5438(03)00004-3

Mauchly, J. W. (1940). Significance Test for Sphericity of a Normal n-Variate Distribution. The Annals of Mathematical Statistics, 11(2), 204-209. https://doi.org/10.1214/aoms/1177731915

McKnight, L., \& Fitton, D. (2010). Touch-screen technology for children: Giving the Right Instructions and Getting the Right Responses. In Proceedings of the 9th International Conference on Interaction Design and Children - IDC '10 (p. 238). New York, New York, USA: ACM Press. https://doi.org/10.1145/1810543.1810580

Mead, G. H. (1934). Play, the game and the generalized other. Mind Self and Society, 152164.

Michel, N., James, J., Iii, C., \& Varela, O. (2009). Active Versus Passive Teaching Styles : An Empirical Study of Student Learning Outcomes. Human Resource Development Quarterly, 20(4). https://doi.org/10.1002/hrdq

Mihajlov, M., Law, E. L.-C., \& Springett, M. (2014). Intuitive Learnability of Touch Gestures for Technology-Naive Older Adults. Interacting with Computers, 27(3), 344-356. https://doi.org/10.1093/iwc/iwu044

Mol, S. E., Bus, A. G., de Jong, M. T., \& Smeets, D. J. H. (2008). Added Value of Dialogic Parent-Child Book Readings: A Meta-Analysis. Early Education and Development, 19(1), 7-26. https://doi.org/10.1080/10409280701838603

Morrow, L. M. (1990). Preparing the classroom environment to promote literacy during play. Early Childhood Research Quarterly, 5(4), 537-554. https://doi.org/10.1016/0885-2006(90)90018-V 
Kindertivity: Usability and Communicability Strategies for Interactive Surfaces and PreKindergarten Children

Moser, T., \& Reikerås, E. (2014). Motor-life-skills of toddlers - a comparative study of Norwegian and British boys and girls applying the Early Years Movement Skills Checklist. European Early Childhood Education Research Journal, 1-21. https://doi.org/10.1080/1350293X.2014.895560

Mumme, D. L., \& Fernald, A. (2003). The infant as onlooker: learning from emotional reactions observed in a television scenario. Child Development, 74(1), 221-237. https://doi.org/10.1111/1467-8624.00532

Nacenta, M. A., Baudisch, P., Benko, H., \& Wilson, A. (2009). Separability of Spatial Manipulations in Multi-touch Interfaces. In Proceedings of Graphics Interface (pp. 175-182). Canadian Information Processing Society.

Nacher, V., Ferreira, A., Jaen, J., \& Garcia-Sanjuan, F. (2016). Are Kindergarten Children Ready for Indirect Drag Interactions? In Proceedings of the 2016 ACM on Interactive Surfaces and Spaces - ISS '16 (pp. 95-101). New York, New York, USA: ACM Press. https://doi.org/10.1145/2992154.2992186

Nacher, V., Garcia-Sanjuan, F., \& Jaen, J. (2016a). Evaluating the Usability of a TangibleMediated Robot for Kindergarten Children Instruction. In 16th IEEE International Conference on Advanced Learning Technologies - ICALT2016. Austin, Texas.

Nacher, V., Garcia-Sanjuan, F., \& Jaen, J. (2016b). Interactive technologies for preschool game-based instruction: Experiences and future challenges. Entertainment Computing, 17, 19-29. https://doi.org/10.1016/j.entcom.2016.07.001

Nacher, V., Garcia-Sanjuan, F., \& Jaen, J. (2018). Evaluating assistive communication languages with kindergarten children on touchscreen devices. International Journal of Human-Computer Interaction, (Under rev.

Nacher, V., \& Jaen, J. (2015a). Evaluating the Accuracy of Pre-kindergarten Children Multi-touch Interaction. In IFIP TC.13 International Conference on HumanComputer Interaction - INTERACT (pp. 549-556). Bamberg. https://doi.org/10.1007/978-3-319-22668-2_42

Nacher, V., \& Jaen, J. (2015b). KINDERTIVITY: Using Interactive Surfaces to Foster Creativity in Pre-kindergarten Children. In Proceedings of the XVI International Conference on Human Computer Interaction - Interacción '15 (pp. 1-2). New York, New York, USA: ACM Press. https://doi.org/10.1145/2829875.2829881

Nacher, V., \& Jaen, J. (2015c). Multi-touch Technology in Early Childhood: Current Trends and Future Challenges. In Proceedings of the XVI International Conference 
on Human Computer Interaction - Interacción '15 (pp. 1-4). New York, New York, USA: ACM Press. https://doi.org/10.1145/2829875.2829887

Nacher, V., Jaen, J., \& Catala, A. (2014). Exploring Visual Cues for Intuitive Communicability of Touch Gestures to Pre-kindergarten Children. In Proceedings of the Ninth ACM International Conference on Interactive Tabletops and Surfaces - ITS '14 (pp. 159-162). New York, New York, USA: ACM Press. https://doi.org/10.1145/2669485.2669523

Nacher, V., Jaen, J., \& Catala, A. (2017). Evaluating Multitouch Semiotics to Empower Prekindergarten Instruction with Interactive Surfaces. Interacting with Computers, 29(2), 97-116. https://doi.org/10.1093/iwc/iww007

Nacher, V., Jaen, J., Catala, A., Navarro, E., \& Gonzalez, P. (2014). Improving PreKindergarten Touch Performance. In Proceedings of the 9th ACM International Conference on Interactive Tabletops and Surfaces (pp. 163-166). New York: ACM. https://doi.org/10.1145/2669485.2669498

Nacher, V., Jaen, J., Navarro, E., Catala, A., \& González, P. (2015). Multi-touch gestures for pre-kindergarten children. International Journal of Human-Computer Studies, 73, 37-51. https://doi.org/10.1016/j.jhcs.2014.08.004

Nacher, V., Jurdi, S., Jaen, J., \& Garcia-Sanjuan, F. (2018). Exploring visual languages for communicating directional awareness to kindergarten children. International Journal of Human-Computer Studies, (Under rev.

Navarro-Newball, A. A., Loaiza, D., Oviedo, C., Castillo, A., Portilla, A., Linares, D., \& Álvarez, G. (2014). Talking to Teo: Video game supported speech therapy. Entertainment Computing, 5(4), 401-412. https://doi.org/10.1016/j.entcom.2014.10.005

Neumann, M. M. (2017). Parent scaffolding of young children's use of touch screen tablets. Early Child Development and Care, 1-11. https://doi.org/10.1080/03004430.2016.1278215

Niemi, H., \& Ovaska, S. (2007). Designing spoken instructions with preschool children. Proceedings of the 6th International Conference on Interaction Design and Children - IDC '07, 133. https://doi.org/10.1145/1297277.1297304

Norman, D. A. (2010). The way I see it: Natural user interfaces are not natural. Interactions, 17(3), 6-10. https://doi.org/10.1145/1744161.1744163 
Kindertivity: Usability and Communicability Strategies for Interactive Surfaces and PreKindergarten Children

Padilla-Zea, N., Sánchez, J. L. G., Vela, F. L. G., Abad-Arranz, A., \& López-Arcos, J. R. (2012). Evaluating emotions in educational videogames. Proceedings of the 13th International Conference on Interacción Persona-Ordenador - INTERACCION '12, 1-3. https://doi.org/10.1145/2379636.2379677

Parten, M. B. (1933). Social play among preschool children. The Journal of Abnormal and Social Psychology, 28(2), 136-147. https://doi.org/10.1037/h0073939

Pecora, N., Murray, J. P., \& Wartella, E. A. (2009). Children and television: Fifty years of research. Routledge.

Pemberton, L., \& Road, L. (2009). Card Sorting Activities with Preschool Children. People and Computers, HCI 2009 -(March 2014), 204-213. https://doi.org/10.1145/1671011.1671036

Piaget, J. (1973). The Child and Reality. New York: Grossman.

Piaget, J. (1975). The Moral Judgment of the Child. New York: The Free Press.

Piper, A. M., O’Brien, E., Morris, M. R., \& Winograd, T. (2006). SIDES: a cooperative tabletop computer game for social skills development. In 20th anniversary conference on Computer Supported Cooperative work (CSCW'06) (pp. 1-10). New York, New York, USA: ACM Press. https://doi.org/10.1145/1180875.1180877

Plato, D. (1955). The Republic. Penguin Books Ltd.

Plowman, L. (2015). Researching Young Children's Everyday Uses of Technology in the Family Home. Interacting with Computers, 27(1), 36-46. https://doi.org/10.1093/iwc/iwu031

Plowman, L., Stevenson, O., Stephen, C., \& McPake, J. (2012). Preschool children's learning with technology at home. Computers \& Education, 59(1), 30-37. https://doi.org/10.1016/j.compedu.2011.11.014

Prates, R. O., de Souza, C. S., \& Barbosa, S. D. J. (2000). Methods and tools: a method for evaluating the communicability of user interfaces. Interactions, 7(1), 31-38. https://doi.org/10.1145/328595.328608

Rädle, R., Jetter, H.-C., Marquardt, N., Reiterer, H., \& Rogers, Y. (2014). HuddleLamp: Spatially-Aware Mobile Displays for Ad-hoc Around-the-Table Collaboration. In Proceedings of the Ninth ACM International Conference on Interactive Tabletops and Surfaces - ITS '14 (pp. 45-54). New York, New York, USA: ACM Press. https://doi.org/10.1145/2669485.2669500 
Rick, J., Francois, P., Fields, B., Fleck, R., Yuill, N., \& Carr, A. (2010). Lo-fi prototyping to design interactive-tabletop applications for children. In 9th International Conference on Interaction Design and Children (IDC'10) (pp. 138-146). New York, New York, USA: ACM Press. https://doi.org/10.1145/1810543.1810559

Rick, J., Harris, A., Marshall, P., Fleck, R., Yuill, N., \& Rogers, Y. (2009). Children designing together on a multi-touch tabletop. In 8th International Conference on Interaction Design and Children (IDC'09) (pp. 106-114). New York, New York, USA: ACM Press. https://doi.org/10.1145/1551788.1551807

Rick, J., \& Rogers, Y. (2008). From DigiQuilt to DigiTile: Adapting educational technology to a multi-touch table. In 3rd IEEE International Workshop on Horizontal Interactive Human Computer Systems (TABLETOP 2008) (pp. 73-80). IEEE. https://doi.org/10.1109/TABLETOP.2008.4660186

Rideout, V. (2011). Zero to Eight: Children's Media Use in America. Common Sense Media. Retrieved from http://vjrconsulting.com/storage/ZerotoEightFINAL2011.pdf

Rubio, G., Navarro, E., \& Montero, F. (2012). APADYT: a multimedia application for SEN learners. Multimedia Tools and Applications, (online first). https://doi.org/10.1007/s11042-012-1304-9

Rushton, S., \& Juola-Rushton, A. (2008). Classroom Learning Environment, Brain Research and The No Child Left Behind Initiative: 6 years Later. Early Childhood Education Journal, 36(1), 87-92. https://doi.org/10.1007/s10643-008-0244-5

Samuelsson, I. P., \& Carlsson, M. A. (2008). The Playing Learning Child: Towards a pedagogy of early childhood. Scandinavian Journal of Educational Research. https://doi.org/10.1080/00313830802497265

Schwarz, J., Xiao, R., Mankoff, J., Hudson, S. E., \& Harrison, C. (2014). Probabilistic palm rejection using spatiotemporal touch features and iterative classification. In Proceedings of the 32nd annual ACM conference on Human factors in computing systems - CHI '14 (pp. 2009-2012). New York, New York, USA: ACM Press. https://doi.org/10.1145/2556288.2557056

Shen, Y., Qiu, Y., Li, K., \& Liu, Y. (2013). Beelight: helping children discover colors. In Proceedings of the 12th International Conference on Interaction Design and Children (pp. 301-304). ACM. https://doi.org/10.1145/2485760.2485813

Shneiderman, B., \& Plaisant, C. (2004). Designing the User Interface: Strategies for Effective Human-Computer Interaction (4th ed.). Boston: Addison-Wesley. 
Kindertivity: Usability and Communicability Strategies for Interactive Surfaces and PreKindergarten Children

Shneiderman, B., Plaisant, C., Cohen, M., \& Jacobs, S. (2009). Designing the User Interface: Strategies for Effective Human-Computer Interaction (5th ed.). Prentice Hall.

Shoukry, L., Sturm, C., \& G.H., G.-E. (2012). Pre-MEGa: A Proposed Framework for the Design and Evaluation of Preschoolers' Mobile Educational Games. In The International Conference on Engineering Education, Instructional Technology, Assessment, and E-learning. Springer.

Shuler, C. (2012). iLearn II: An Analysis of the Education Category of the iTunes App Store. New York: The Joan Ganz Cooney Center at Sesame Workshop. Retrieved from http://www.joanganzcooneycenter.org/wp-content/uploads/2012/01/ilearnii.pdf

Sims, C., \& Colunga, E. (2005). Parent-Child Screen Media Co-Viewing: Influences on Toddlers' Word Learning and Retention. In Proceedings of the 35th Annual Conference of the Cognitive Science Society (pp. 1324-1329). Austin, Texas. Retrieved from http://mindmodeling.org/cogsci2013/papers/0252/paper0252.pdf

Singer, D. G., Golinkoff, R. M., \& Hirsh-Pasek, K. (2006). Play = Learning: How Play Motivates and Enhances Children's Cognitive and Social-Emotional Growth. New York: Oxford University Press. https://doi.org/10.1093/acprof:oso/9780195304381.001.0001

Sluis, R. J. W., Weevers, I., van Schijndel, C. H. G. J., Kolos-Mazuryk, L., Fitrianie, S., \& Martens, J. B. O. S. (2004). Read-It: Five-to-seven-year-old children learn to read in a tabletop environment. In 2004 conference on Interaction design and children building a community (IDC'04) (pp. 73-80). New York, New York, USA: ACM Press. https://doi.org/10.1145/1017833.1017843

Smith, S. P., Burd, E., \& Rick, J. (2012). Developing, evaluating and deploying multi-touch systems. International Journal of Human-Computer Studies, 70(10), 653-656. https://doi.org/10.1016/j.ijhcs.2012.07.002

Soute, I., \& Nijmeijer, H. (2014). An Owl in the Classroom: Development of an Interactive Storytelling Application for Preschoolers. In Proceedings of the 2014 conference on Interaction design and children (pp. 261-264). ACM. https://doi.org/10.1145/2593968.2610467

Steurer, P., \& Srivastava, M. B. (2003). System design of Smart Table. Proceedings of the First IEEE International Conference on Pervasive Computing and Communications (PerCom 2003), 473-480. https://doi.org/10.1109/PERCOM.2003.1192772 
Strawhacker, A., \& Bers, M. U. (2014). "I want my robot to look for food": Comparing Kindergartner's programming comprehension using tangible, graphic, and hybrid user interfaces. International Journal of Technology and Design Education. https://doi.org/10.1007/s10798-014-9287-7

Strommen, E. F., Revelle, G. L., Medoff, L. M., \& Razavi, S. (1996). Slow and steady wins the race? Three-year-old children and pointing device use. Behaviour and Information Technology, 15(1), 57-64. https://doi.org/10.1083/jcb1666rr4

Subban, P. (2006). Differentiated instruction: A research basis. International Education Journal, 7(7), 935-947. https://doi.org/10.1111/j.1365-2648.2006.04074.x

Tanaka, F., Fortenberry, B., Aisaka, K., \& Movellan, J. R. (2005). Plans for Developing Real-time Dance Interaction between QRIO and Toddlers in a Classroom Environment. In Procceedings on the 4th International Conference on Development and Learning (pp. 142-147). IEEE. https://doi.org/10.1109/DEVLRN.2005.1490963

Tanaka, F., \& Matsuzoe, S. (2012). Learning Verbs by Teaching a Care-Receiving Robot by Children: An Experimental Report. In Proceedings of the 7th annual ACM/IEEE international conference on Human-Robot Interaction (pp. 253-254). ACM. https://doi.org/10.1145/2157689.2157781

Tanaka, F., \& Takahashi, T. (2012). A tricycle-style teleoperational interface that remotely controls a robot for classroom children. In Proceedings of the 7th annual ACM/IEEE international conference on Human-Robot Interaction (pp. 255-256). https://doi.org/10.1145/2157689.2157782

Terra, D., Brinkman, W. P., \& Heynderickx, I. (2009). Ease-of-Use and Enjoyment of Traditional vs. Stylus Input for Children in a Brazilian Primary School. In LatinDisplay (pp. 151-155).

Thomas, G. V., Nye, R., \& Robinson, E. J. (1994). How children view pictures: Children's responses to pictures as things in themselves and as representations of something else. Cognitive Development, 9(2), 141-164. https://doi.org/10.1016/08852014(94)90001-9

Tyng, K. S., Zaman, H. B., \& Ahmad, A. (2011a). Visual Application in Multi-touch Tabletop for Mathematics Learning: A Preliminary Study. In 2nd International Conference on Visual Informatics: sustaining research and innovations (IVIC'11) (pp. 319-328). Springer.

Tyng, K. S., Zaman, H. B., \& Ahmad, A. (2011b). Visual Application in Multi-touch 
Kindertivity: Usability and Communicability Strategies for Interactive Surfaces and PreKindergarten Children

Tabletop for Mathematics Learning: A Preliminary Study. In 5th International Conference on E-learning and Games (Edutainment 2010) (Vol. 6249, pp. 319-328). Springer. https://doi.org/10.1007/978-3-642-25191-7_31

van Daal, V., \& Reitsma, P. (2000). Computer-assisted learning to read and spell: results from two pilot studies. Journal of Research in Reading, 23(2), 181-193. https://doi.org/10.1111/1467-9817.00113

Van Der Meij, H., \& Van Der Meij, J. (2014). A comparison of paper-based and video tutorials for software learning. Computers and Education, 78, 150-159. https://doi.org/10.1016/j.compedu.2014.06.003

Vatavu, R., Cramariuc, G., \& Schipor, D. M. (2015). Touch interaction for children aged 3 to 6 years: Experimental findings and relationship to motor skills. International Journal of Human-Computer Studies, 74, 54-76. https://doi.org/10.1016/j.ijhcs.2014.10.007

Veitch, J., Bagley, S., Ball, K., \& Salmon, J. (2006). Where do children usually play? A qualitative study of parents' perceptions of influences on children's active free-play. Health \& Place, 12(4), 383-393. https://doi.org/10.1016/j.healthplace.2005.02.009

Vogel, D., \& Casiez, G. (2012). Hand occlusion on a multi-touch tabletop. In Proceedings of the 2012 ACM annual conference on Human Factors in Computing Systems - CHI '12 (p. 2307). New York, New York, USA: ACM Press. https://doi.org/10.1145/2207676.2208390

Wakefield, J., \& Smith, D. (2012). From Socrates to Satellites: iPad Learning in an Undergraduate Course. Creative Education, 03(05), 643-648. https://doi.org/10.4236/ce.2012.35094

Walter, R., Bailly, G., \& Müller, J. (2013). StrikeAPose: revealing mid-air gestures on public displays. In Proceedings of the SIGCHI Conference on Human Factors in Computing Systems - CHI '13 (pp. 841-850). New York, New York, USA: ACM Press. https://doi.org/10.1145/2470654.2470774

Weiss, P. L., Gal, E., Eden, S., Zancanaro, M., \& Telch, F. (2011). Usability of a MultiTouch Tabletop Surface to Enhance Social Competence Training for Children with Autism Spectrum Disorder. In Chais conference on instructional technologies research 2011: Learning in the technological era (CHAIS 2011) (pp. 71-78). The Open University of Israel.

Wieringa, R. (2009). Design science as nested problem solving. In Proceedings of the 4th 
International Conference on Design Science Research in Information Systems and Technology - DESRIST '09 (p. 1). New York, New York, USA: ACM Press. https://doi.org/10.1145/1555619.1555630

Willis, J., Weiser, B., \& Kirkwood, D. (2014). Bridging the Gap: Meeting the Needs of Early Childhood Students by Integrating Technology and Environmental Education. International Journal of Early Childhood, 2(1), 140.

Wolock, E., Ann Orr, E. D., \& Buckleitner, W. (2006). Child development 101 for the developers of interactive media. Active Learning Associates, Inc. Retrieved from http://www.childrenssoftware.com/pdf/dustormagic_handout06.pdf

Yu, X., Zhang, M., Ren, J., Zhao, H., \& Zhu, Z. (2010). Experimental Development of Competitive Digital Educational Games on Multi-touch Screen for Young Children. In 5th International Conference on E-learning and Games (Edutainment 2010) (Vol. 6249, pp. 367-375). Springer.

Zaranis, N., Kalogiannakis, M., \& Papadakis, S. (2013). Using Mobile Devices for Teaching Realistic Mathematics in Kindergarten Education. Creative Education, 04(07), 1-10. https://doi.org/10.4236/ce.2013.47A1001

Zhao, J., Soukoref, R. W., \& Balakrishnan, R. (2011). A Model of Multi-touch Manipulation. In Graphisme, animation et nouveaux medias (GRAND 2011). Vancouver, BC, Canada. $\quad$ Retrieved from http://soukoreff.com/academic/grand2011.pdf 



\section{List of Figures}

Figure 1. Problem decomposition (in rectangles) and the chapter in which they are dealt with (in circles).

Figure 2. Example screen of the activity spelling a word by its sound (from (van Daal

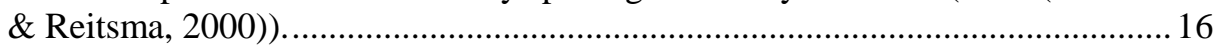

Figure 3. Mini-games examples of Talking to Teo game (from (Navarro-Newball et al., 2014)). 17

Figure 4. (a) MEL-Vis from (Tyng et al., 2011a). (b) The Teaching Table from (Khandelwal \& Mazalek, 2007).

Figure 5. Children playing in the The Farm Game (Marco, Cerezo, Baldasarri, et al., 2009; Marco, Cerezo, \& Baldassarri, 2009).

Figure 6. Child interacting with CamQuest (extracted from (Berggren \& Hedler, 2014))

Figure 7. Child performing simple and double drag gestures (extracted from (Vatavu et al., 2015)).

Figure 8. Description of the animated hand (top), the animated object (middle) and the iconic (bottom) language for the scale up gesture (extracted from (Nacher et al., 2017)).

Figure 9. (a) Owl-shaped robot (extracted from (Soute \& Nijmeijer, 2014)). (b)

Beelight (extracted from (Shen et al., 2013)).

Figure 10. (a) Tricycle interface (extracted from (Tanaka \& Takahashi, 2012)). (b) Children dancing with QRIO (extracted from (Tanaka et al., 2005))

Figure 11. Children driving a mobile robot (extracted from (Garcia-Sanjuan, Jaen, Nacher, et al., 2015)).

Figure 12. The Code-a-Pillar robot presented by Fisher-Price. 26 
Kindertivity: Usability and Communicability Strategies for Interactive Surfaces and PreKindergarten Children

Figure 13. The Kidroom from the three cameras used to sensor (extracted from (Bobick et al., 1999)).

Figure 14. The backpack used for tracking in (Hwang et al., 2012). .30

Figure 15. Target age 2009 vs 2011 for Education cat. in the Apple Store (Shuler, 2012).

Figure 16. Examples of scale up and drag tests (extracted from(Nacher et al., 2015)). 36

Figure 17. Child performing double drag gestures (extracted from (Vatavu et al., 2015)).

Figure 18. Description of the animated visual (top) and the iconic (bottom) language for the scale up gesture (extracted from (Nacher, Jaen, \& Catala, 2014))

Figure 19. Learning methods. .54

Figure 20. Supported interactions 54

Figure 21. Example of a simple tap, double tap or long pressed test 57

Figure 22. Example of a drag test. 58

Figure 23. Example of a scale test: (a) scale up (b) scale down 59

Figure 24. Example of a One-Finger rotation test 59

Figure 25. Example of a Two-Finger rotation test .60

Figure 26. Mean completion time by task and by age group. Error bars reflect standard error.

Figure 27. Mean completion time by task and by gender (F: Female, M: Male) .62

Figure 28. Success rate by task and age group . .64

Figure 29. Success rate by task and gender (F: Female, M: Male) .64

Figure 30. Users grouped by number of successfully performed tasks . .66

Figure 31. Success rate by task . .67 
Figure 32. Examples of actual scale (left) and drag (right) gestures ............................71

Figure 33. Example of long pressed and double tap task.............................................. 86

Figure 34. Success rate by maximum allowed time between taps in milliseconds ...........88

Figure 35. Example tests: (a) drag (b) rotation (c) scale up (d) scale down..................95

Figure 36. Pre-kindergarten child performing the drag task. .......................................98

Figure 37. Example of a task with two obstacles for the drag, accelerometer, tap (top)

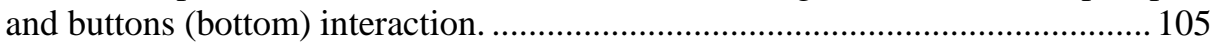

Figure 38. Success rate by interaction mechanism and age group............................. 107

Figure 39. Completion time by interaction mechanism and age group....................... 107

Figure 40. Collisions by interaction mechanism and age group............................... 108

Figure 41. Kindergarten child performing the accelerometer task.............................. 110

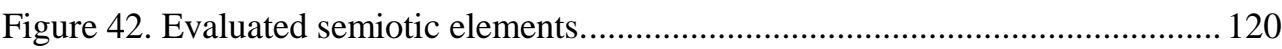

Figure 43. Description of animated visual cues and icons used in the considered gesture

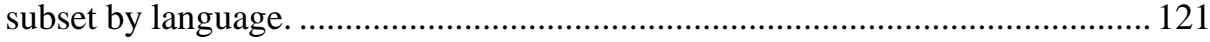

Figure 44. The image chosen by instructors for the Animated Hand language. ......... 132

Figure 45. An example of the animated object language for the drag gesture............. 132

Figure 46. The three available commercial iconic languages (scale up gesture)........ 133

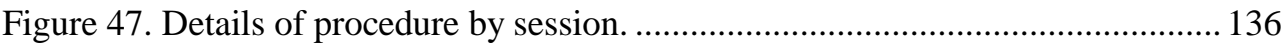

Figure 48. Examples of communication of the tap gesture for each language. Top: Animated Hand language; Center: Animated Object language; Bottom: Iconic

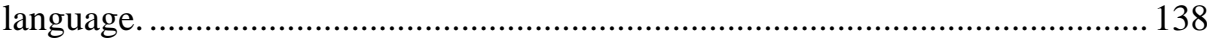

Figure 49. Examples of communication of a long pressed gesture for each language. Top: Animated Hand language; Center: Animated Object language; Bottom: Iconic language. 
Kindertivity: Usability and Communicability Strategies for Interactive Surfaces and PreKindergarten Children

Figure 50. Examples of communication of a drag gesture for each language. Top: Animated Hand language; Center: Animated Object language; Bottom: Iconic language.

Figure 51. Examples of communication of a rotation gesture for each language. Top: Animated Hand language; Center: Animated Object language; Bottom: Iconic language.

Figure 52. Examples of communication of a scale up gesture for each language. Top: Animated Hand language; Center: Animated Object language; Bottom: Iconic language.

Figure 53. Examples of communication of a scale down gesture for each language.

Top: Animated Hand language; Center: Animated Object language; Bottom:

Iconic language.

Figure 54. Success rate by phase and gesture

Figure 55. Success percentage by language and gesture.

Figure 56. Success rate by phase and gesture for the animated languages. Top:

Animated Hand; Bottom: Animated Object.

Figure 57. Success rate by phase and gesture for the iconic language.

Figure 58. Success percentage by gender and gesture.

Figure 59. Success percentage by gender and task for the iconic language

Figure 60. Success percentage by gender and task for the animated languages. Top:

Animated Hand; Bottom: Animated Object

Figure 61 . Success percentage by age group and gesture

Figure 62. Success percentage by age group and task for the animated languages. Top:

Animated Hand; Bottom: Animated Object.

Figure 63. Success percentage by age group and task for the iconic language

Figure 64. Example of communication used in a commercial application. 165

Figure 65. Example of communication used in a commercial application. 166 
Figure 66. Example of the task with the mini-map (a) abstraction (b) application snapshot......

Figure 67. Example of the task with the thumbnail language (a) abstraction (b) application snapshot.

Figure 68. Example of the task with the arrow language (a) abstraction (b) application snapshot.

Figure 69. Example of some objects of the three diffrent topics to pick up in the game (animals, fruits and jobs)

Figure 70. Task example without visual language. Topic: Fruits. 171

Figure 71 . Success rate by visual language and age group. 173

Figure 72. Completion time by visual language and age group 174

Figure 73. Increment of distance travelled by visual language and age group. 175

Figure 74. Changes of direction by age group and visual language 176

Figure 75. Number of collisions by visual language and age group. 177

Figure 76. Number of incorrect pickups by visual language and age group. 178

Figure 77. Representation of the thumbnail visual language. 194

Figure 78. Example of the game in the Exploration \& Curiosity stage with an active bridge and the visual language representing a scale up gesture. 196

Figure 79. Example of the game in the Problem-solving Reasoning stage for the lion.

Figure 80. Example of the Observation stage for the lion. 198

Figure 81 . Time spent in each stage of the task in percentage. 200

Figure 82. Percentage of time spent in each stage by age group 201

Figure 83. Percentage of the available animals visited during the experiment by age group, semiotic density and gender. 
Kindertivity: Usability and Communicability Strategies for Interactive Surfaces and PreKindergarten Children

Figure 84 . Total animals visited in the task by age, semiotic density and gender.......202

Figure 85 . Percentage of gestures fitted by age, semiotic density and gender. 203

Figure 86. Kindergarteners' success rate achieved by gesture when applying design guidelines in the Double Tap and Long Pressed gestures. 


\section{List of Tables}

Table 1. Comparison of works .28

Table 2. Main features of the experiment regarding completion time ..........................62

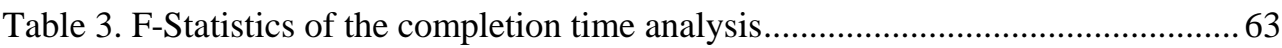

Table 4. Main features of the experiment regarding degree of success ...................... 65

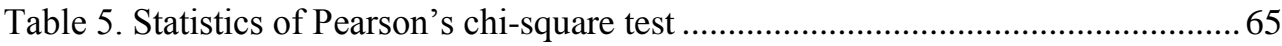

Table 6. Main features success in the experimental tests.......................................6 67

Table 7. Task comparison by success with Pearson's chi-square test of independence $\chi^{2}$ ( $\mathrm{DoF}=1, \mathrm{~N}=32$ ).

Table 8. Variance of completion time (in seconds) by task......................................6 68

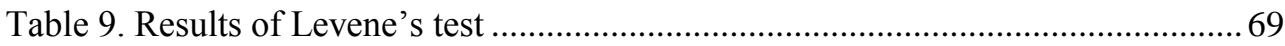

Table 10. Time in milliseconds to perform the tasks by age .................................. 79

Table 11. Time in milliseconds to perform the tasks by gender ................................. 80

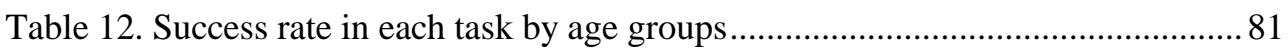

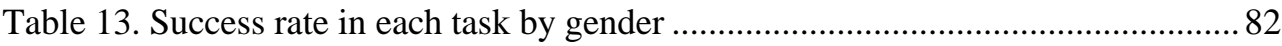

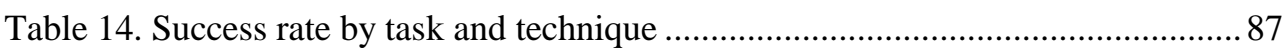

Table 15. Completion time (msec.) by task and technique ...................................... 88

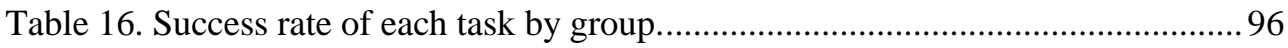

Table 17. Completion time in miliseconds of each task by group. ........................... 97 
Kindertivity: Usability and Communicability Strategies for Interactive Surfaces and PreKindergarten Children

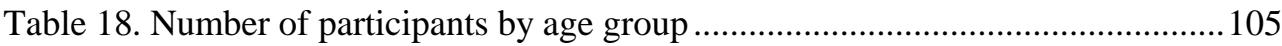

Table 19. Success by language, gender by phase for each task. (P1= Intuitive Phase, P2=Immediate Recall Phase)

Table 20. P-value of the post-hoc pair-wise comparison of sessions by task (Bonferroni

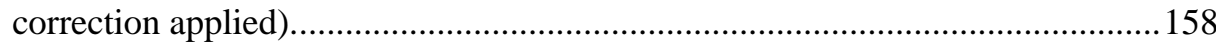

Table 21. P-value of the multiple comparison between languages by tasks (Bonferroni

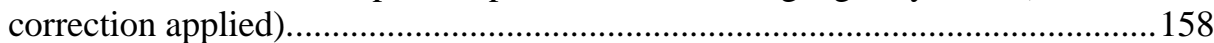

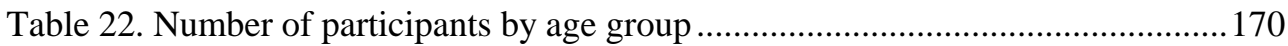

Table 23. Success rate by visual language and age group..........................................184

Table 24. Completion time by visual language and age group (s)...........................184

Table 25. Percentage of increment in the travelled path in comparison with the ideal.

Table 26. Changes of direction by visual language and age group............................185

Table 27. Number of collisions with blocks by visual language and age group. .........186

Table 28. Number of incorrect pickups by visual language and age..........................186

Table 29. P-value of the post-hoc pair-wise comparison of visual languages for all the

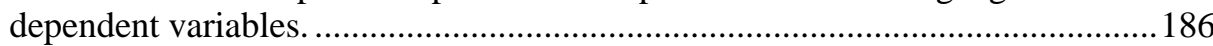

Table 30. Statistics of the conducted ANOVA for all the dependent variables. .........210

Table 31. Value of the evaluated dependent variables by age group. .........................210

Table 32. Value of the evaluated dependent variables by semiotic density................211

Table 33. Value of the evaluated dependent variables by gender. ..............................211 